\title{
Synthetic Studies towards 1,2,3,3a,4,8b-hexahydropyrrolo[3,2-b]indole core. Unusual Fragmentation with 1,2-Aryl Shift
}

\author{
Alexander V. Aksenov, ${ }^{\dagger},{ }^{*}$ Elena V. Aleksandrova,${ }^{\dagger}$ Dmitrii A. Aksenov,$^{\dagger}$ Anna A. Aksenova, ${ }^{\dagger}$ \\ Nicolai A. Aksenov, ${ }^{\dagger}$ Mezvah A. Nobi, ${ }^{\dagger}$ Michael Rubin. ${ }^{\dagger}, * *$ \\ $\dagger$ Department of Chemistry, North Caucasus Federal University, 1a Pushkin St., Stavropol 355009, Russian \\ Federation; E-mailaaksenov@ncfu.ru \\ *Department of Chemistry, University of Kansas, 1567 Irving Hill Road-Lawrence, KS 66045, USA. E-mail \\ mrubin@ku.edu
}

\section{Supporting Information}

\section{Table of content}

${ }^{1} \mathrm{H}$ and ${ }^{13} \mathrm{C}\left\{{ }^{1} \mathrm{H}\right\}$ Spectral Charts for 5-Isopropyl-1-methyl-2-phenyl-1H-indole 2w........ S2

${ }^{1} \mathrm{H}$ and ${ }^{13} \mathrm{C}\left\{{ }^{1} \mathrm{H}\right\}$ Spectral Charts for $4^{\prime} H$-spiro[indole-3,5'-isoxazoles] 3.................... $\mathbf{S 4}$

${ }^{1} \mathrm{H}$ and ${ }^{13} \mathrm{C}\left\{{ }^{1} \mathrm{H}\right\}$ Spectral Charts for 2-(3-oxoindolin-2-yl)acetonitriles 4................. S18

${ }^{1} \mathrm{H}$ and ${ }^{13} \mathrm{C}\left\{{ }^{1} \mathrm{H}\right\}$ Spectral Charts for 1,3a,4,8b-tetrahydropyrrolo[3,2-b]indol-2(1H)-ones

5............................................................................. $\mathbf{S 4 2}$

${ }^{1} \mathrm{H}$ and ${ }^{13} \mathrm{C}\left\{{ }^{1} \mathrm{H}\right\}$ Spectral Charts for 3-hydroxyindolin-2-ones 6.......................... S60

${ }^{1} \mathrm{H}$ and ${ }^{13} \mathrm{C}\left\{{ }^{1} \mathrm{H}\right\}$ Spectral Charts for 2-benzamidobenzoic acids 13........................ S90

HRMS Charts................................................................... $\mathbf{S 9 4}$

HRMS Charts for 5-isopropyl-1-methyl-2-phenyl-1H-indole 2w..................... S94

HRMS Charts for 4 ' $H$-spiro[indole-3,5'-isoxazoles] 3............................... S95

HRMS Charts for 2-(3-oxoindolin-2-yl)acetonitriles 4............................... S97

HRMS Charts for $1,3 a, 4,8 b$-tetrahydropyrrolo[3,2-b]indol-2(1H)-ones 5............... S100

HRMS Charts for 3-hydroxyindolin-2-ones 6.................................... S102

X-Ray crystallography data for 5 p............................................... S105

X-Ray crystallography data for 6 a.................................................... S113

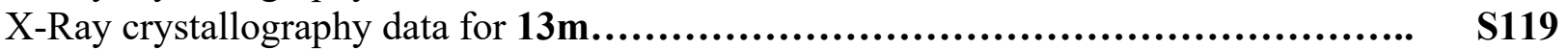

References................................................................................. S128 
${ }^{1} \mathrm{H}$ and ${ }^{13} \mathrm{C}\left\{{ }^{1} \mathrm{H}\right\}$ Spectral Charts for 5-Isopropyl-1-methyl-2-phenyl-1H-indole (2w)

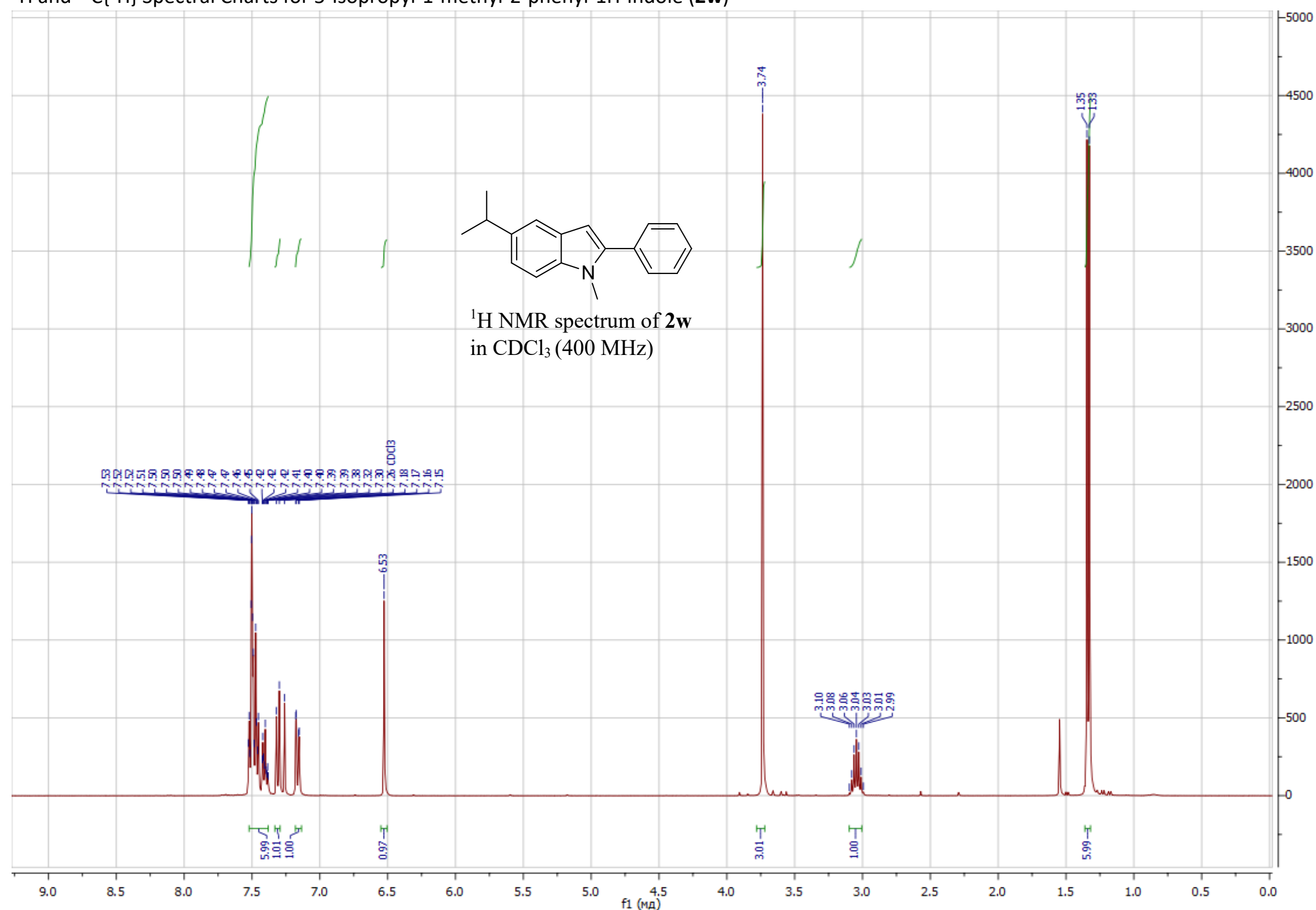




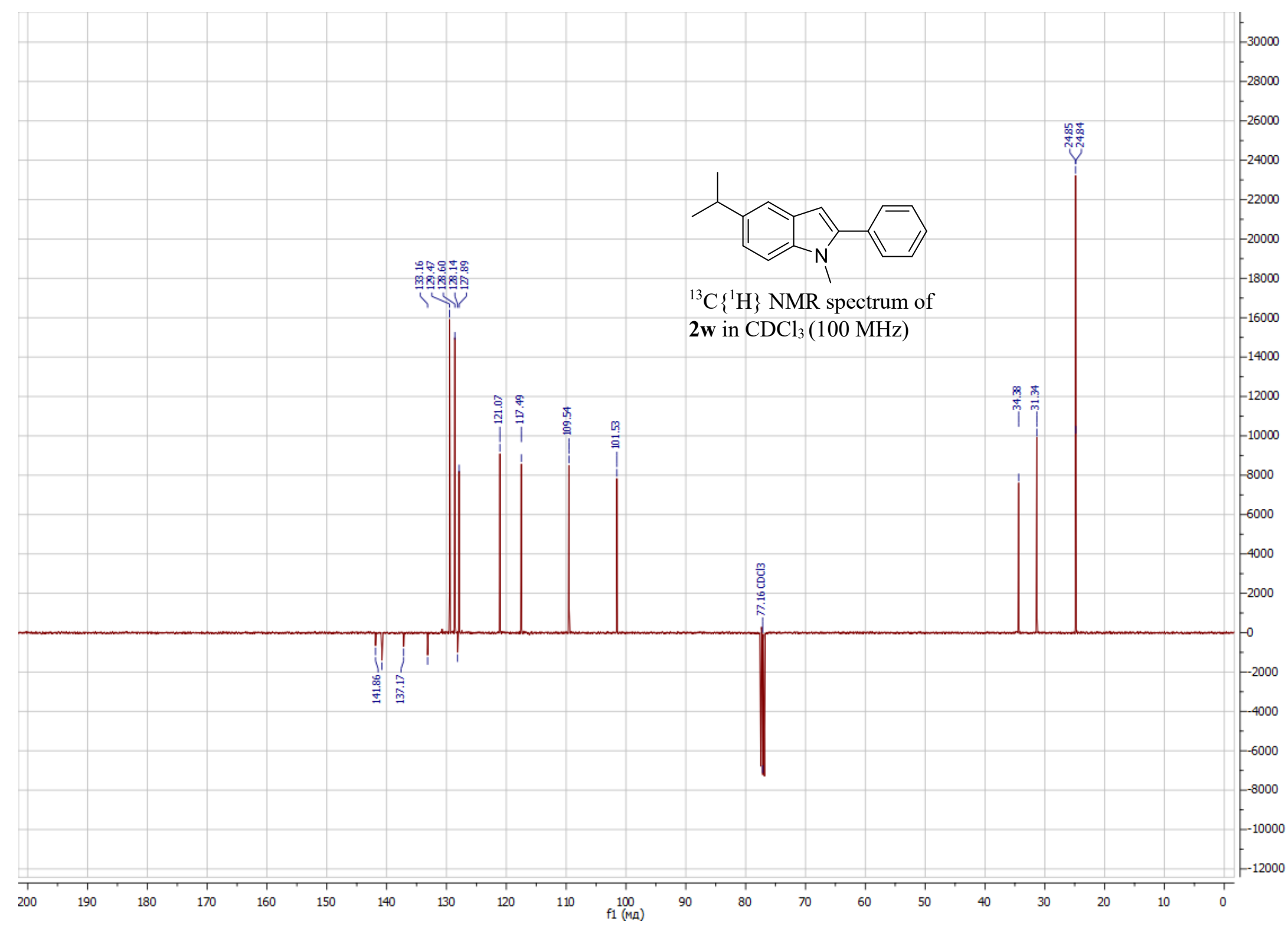


${ }^{1} \mathrm{H}$ and ${ }^{13} \mathrm{C}$ Spectral Charts for 4 'H-spiro[indole-3,5'-isoxazoles] 3

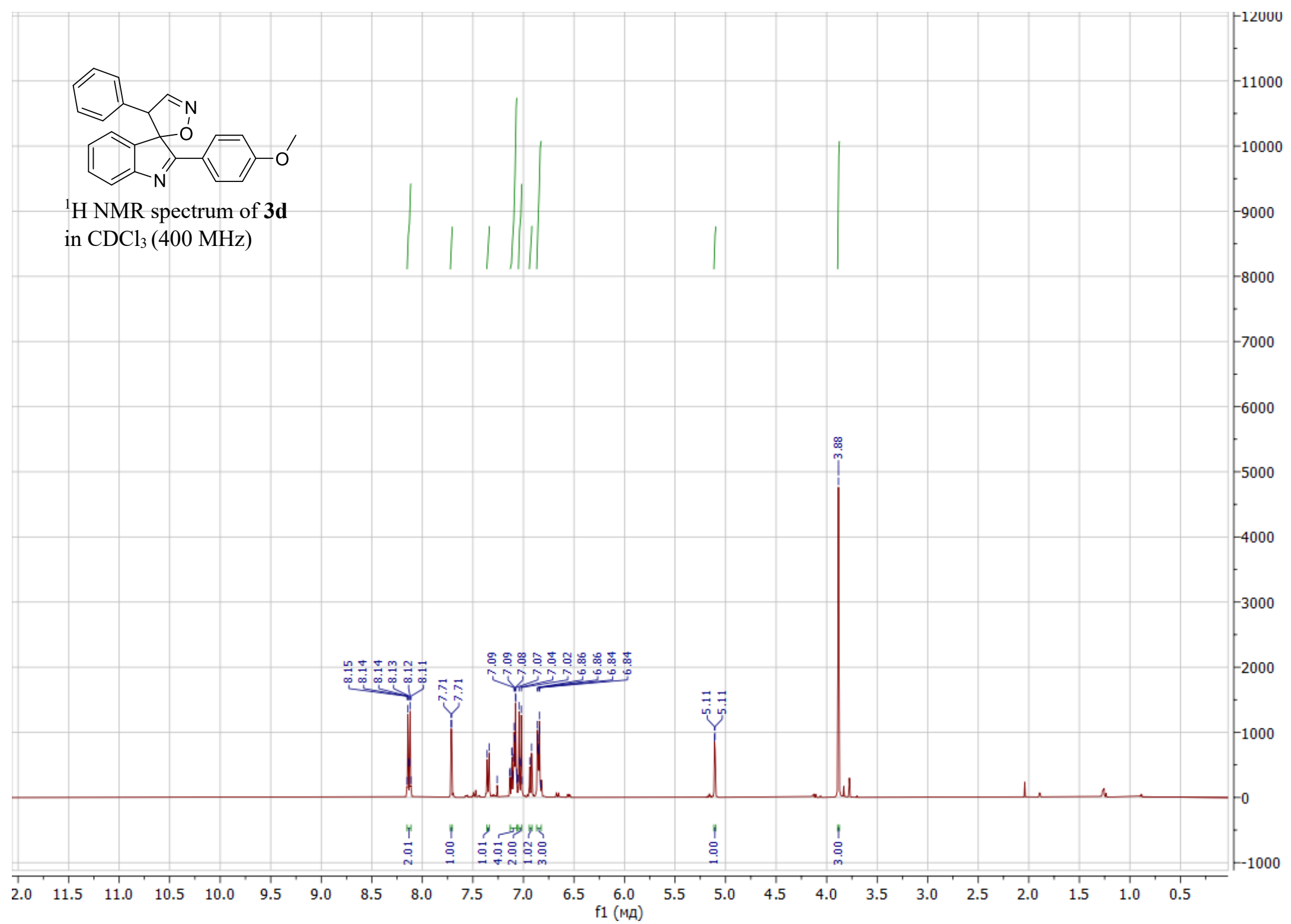




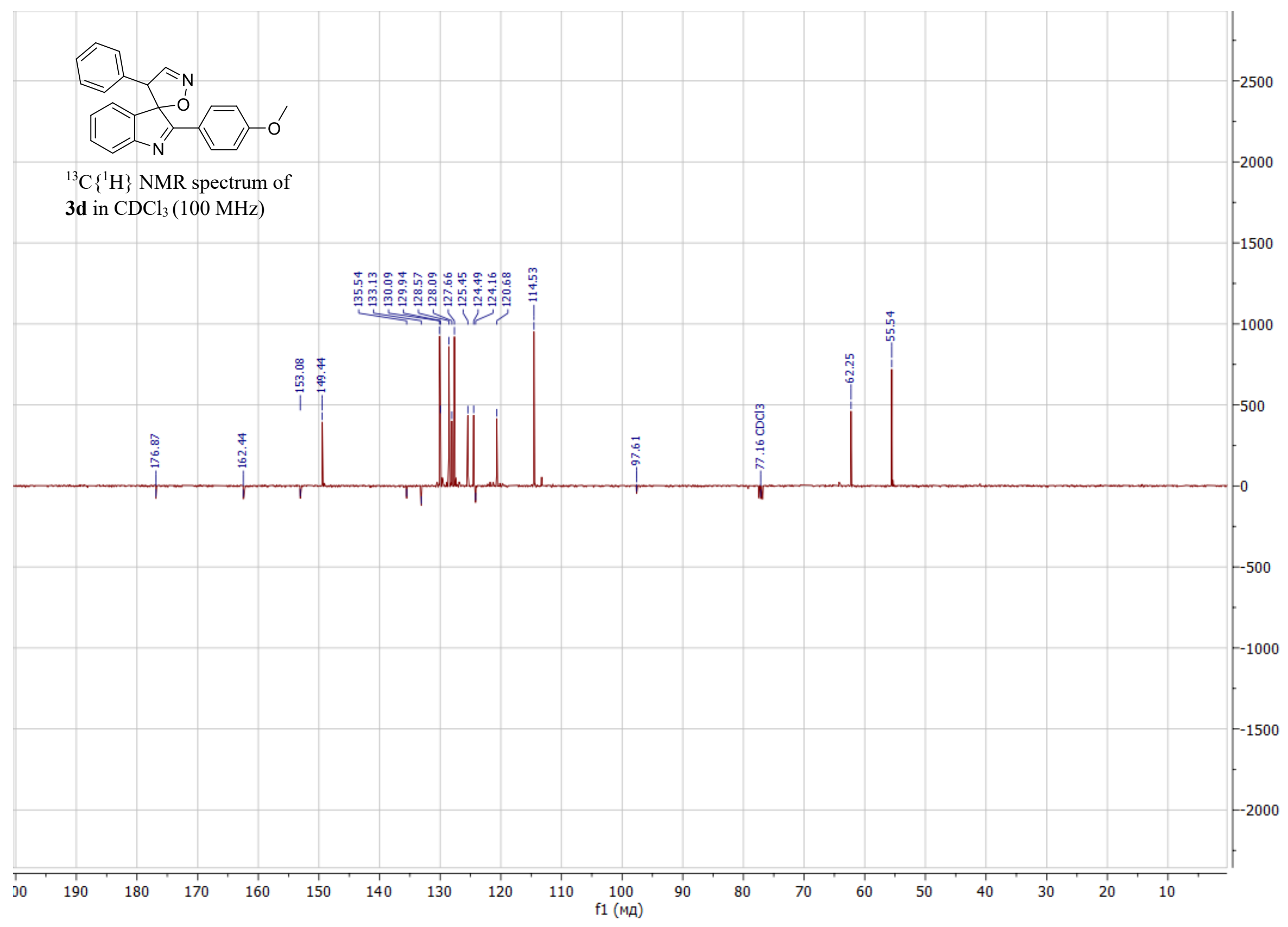




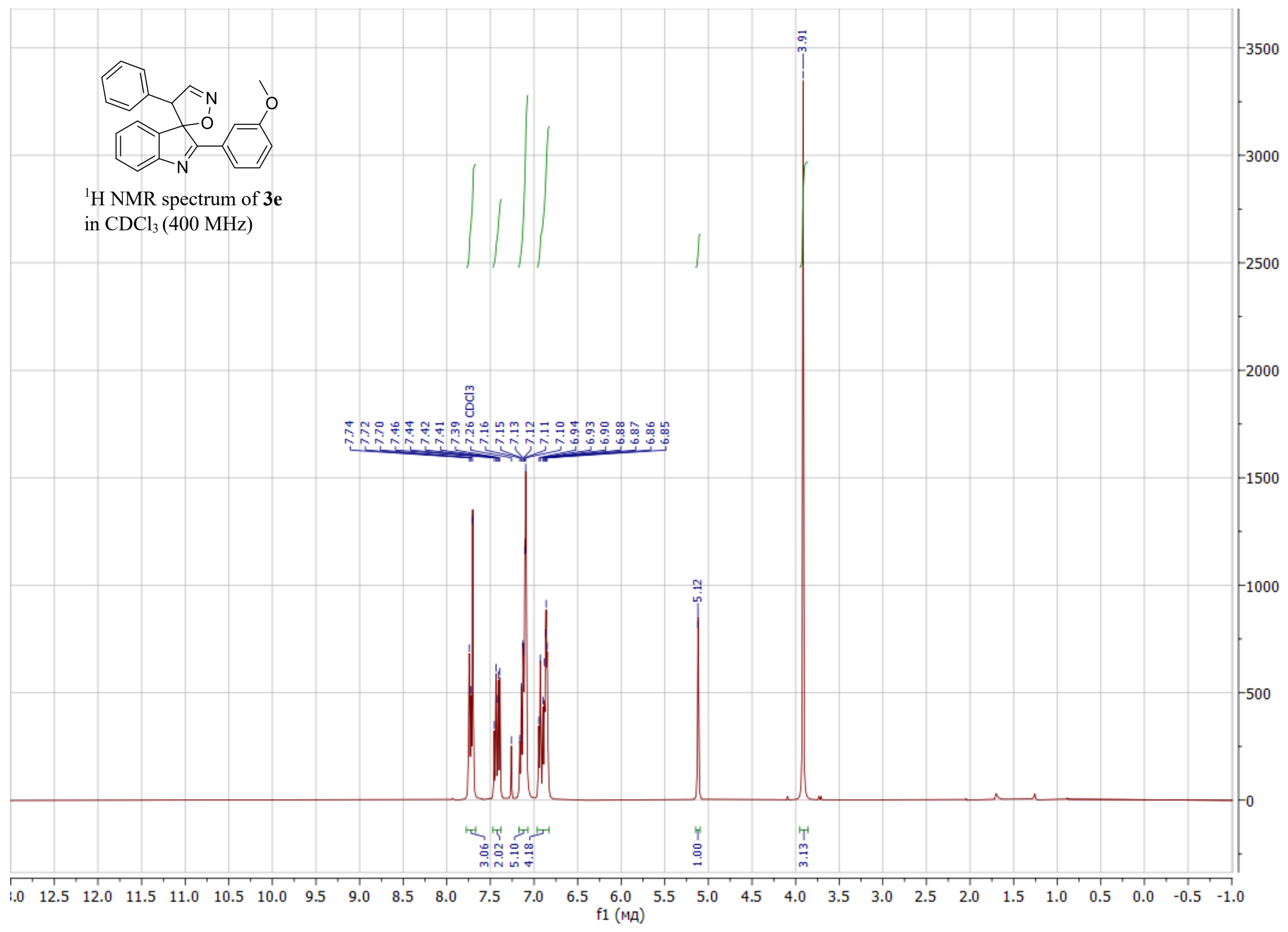




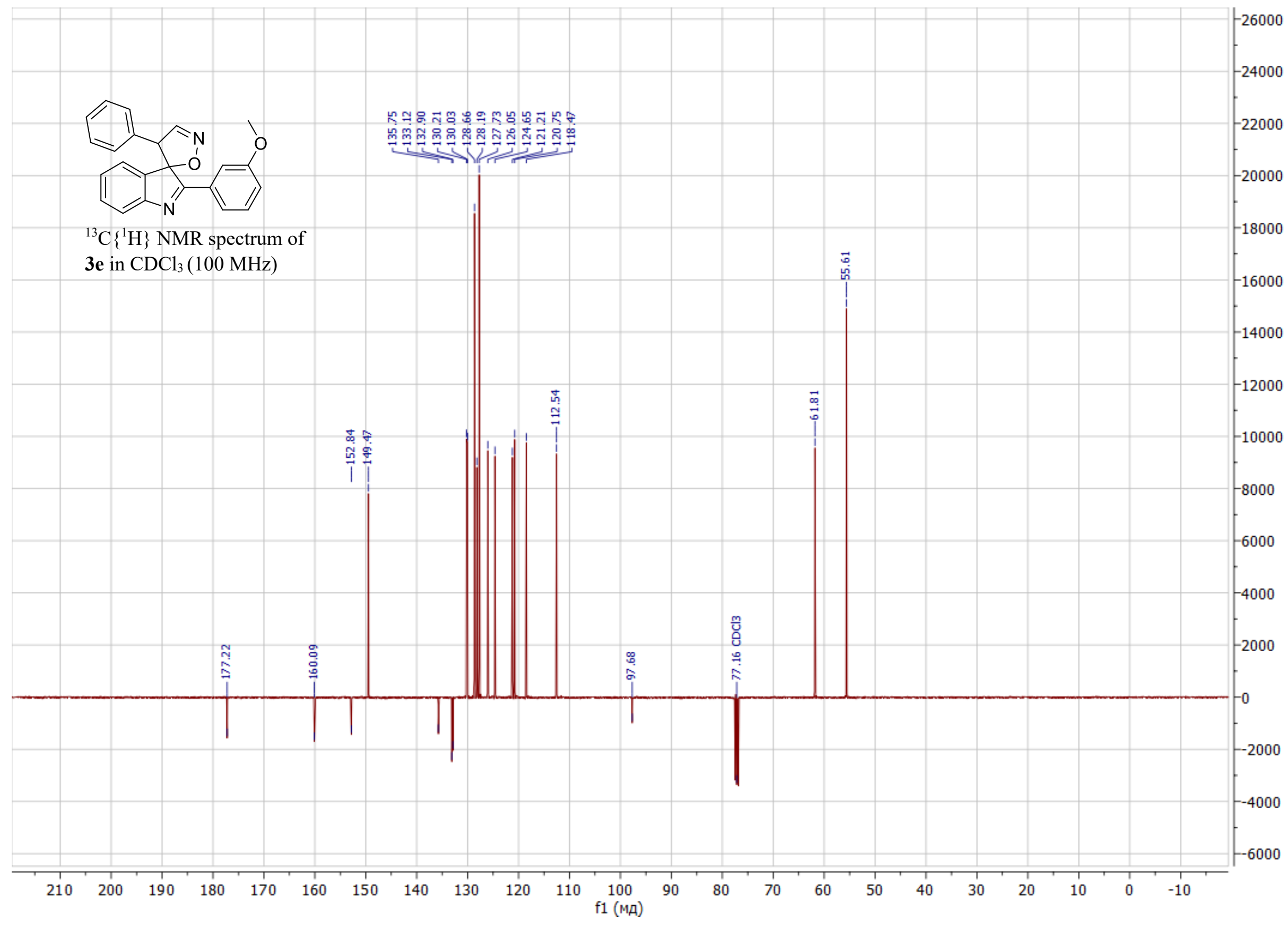




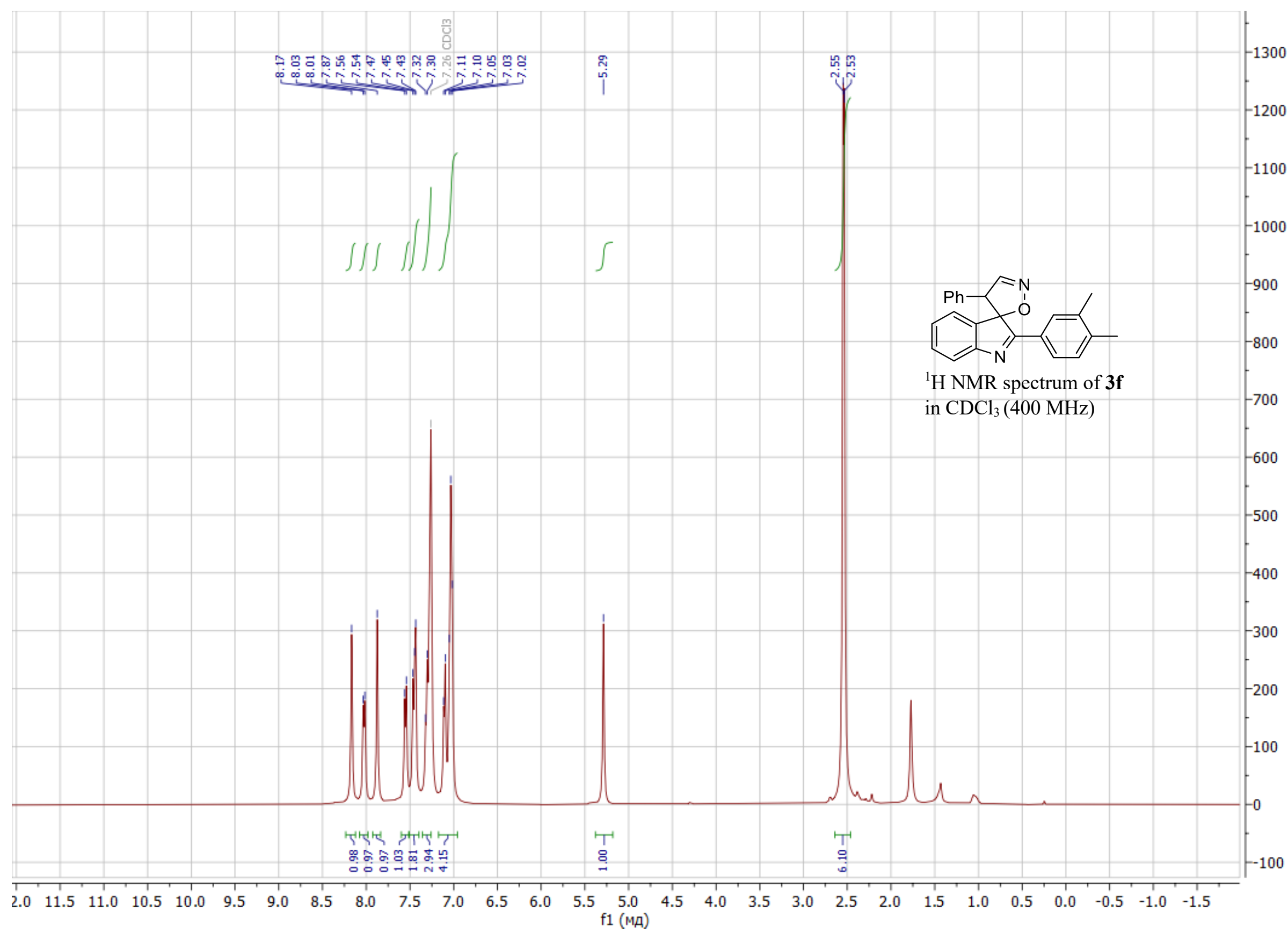




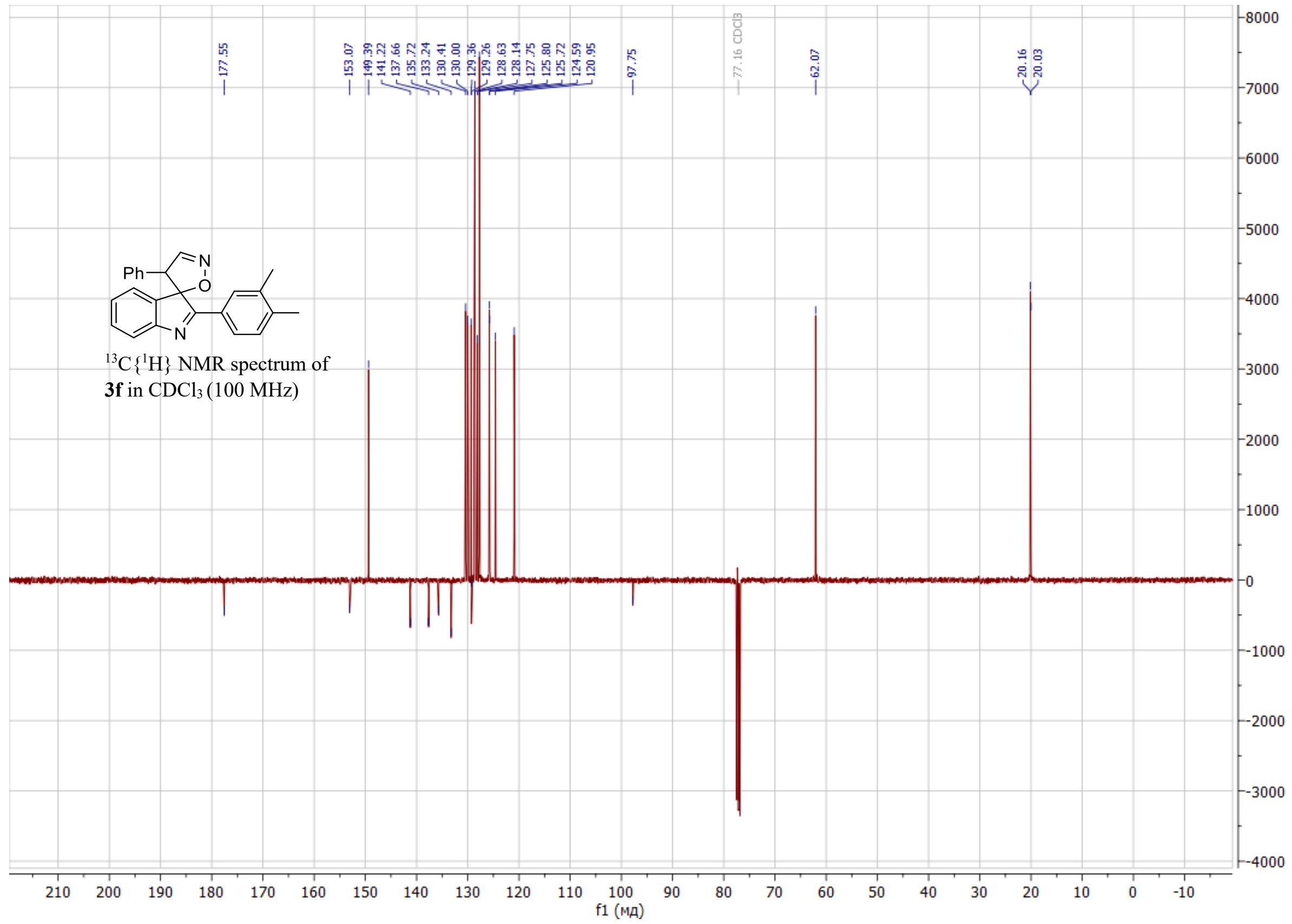




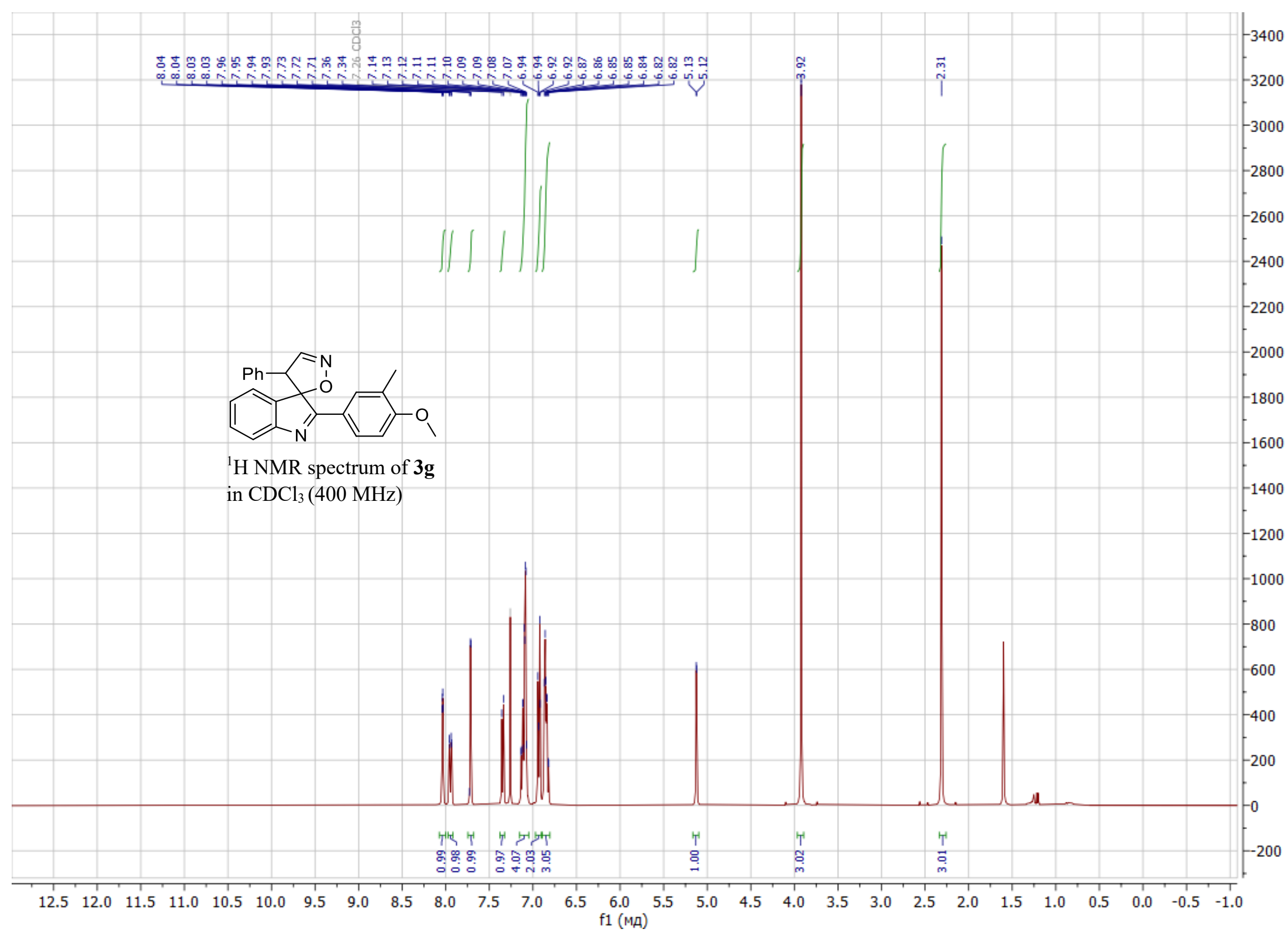




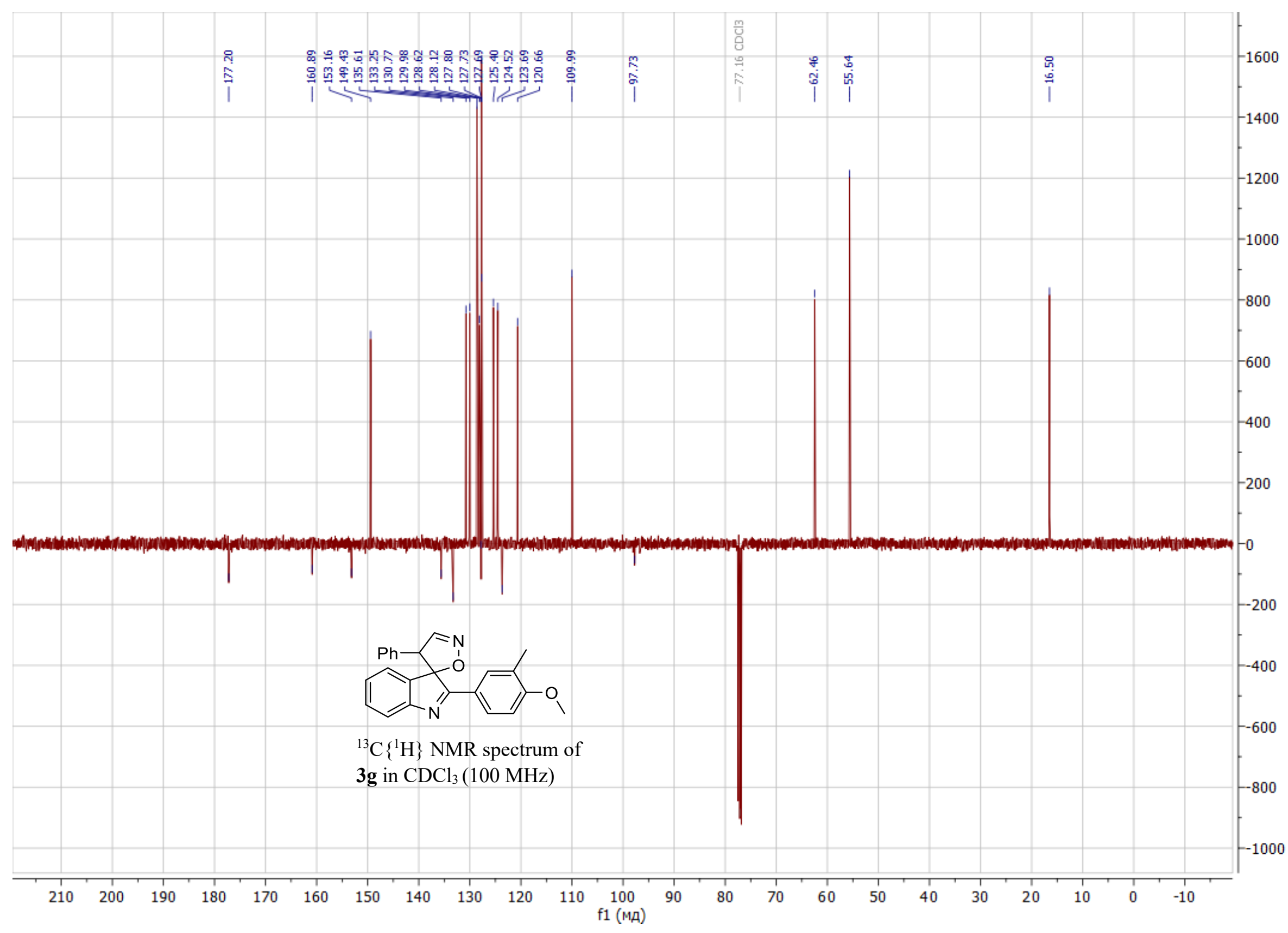




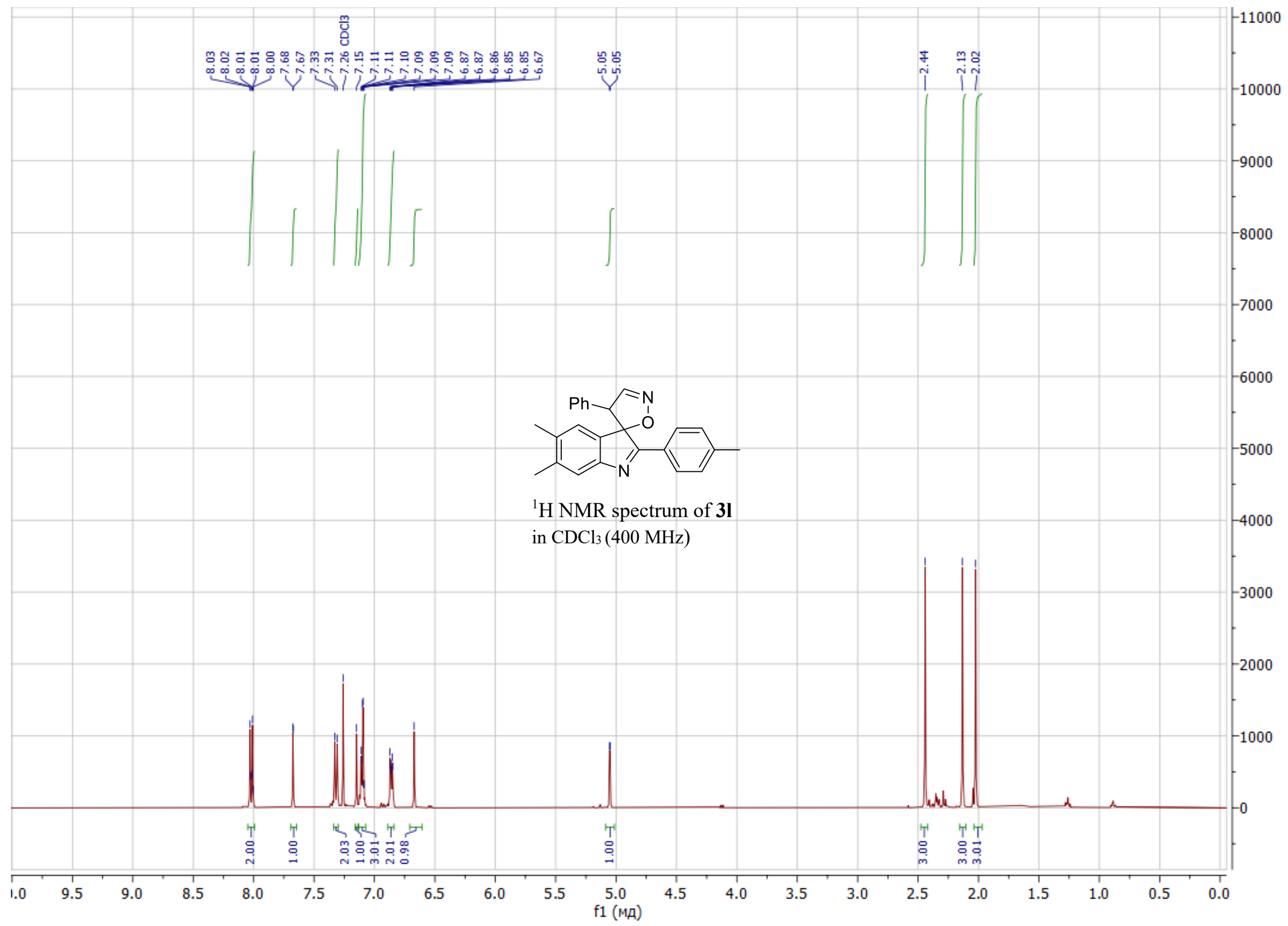




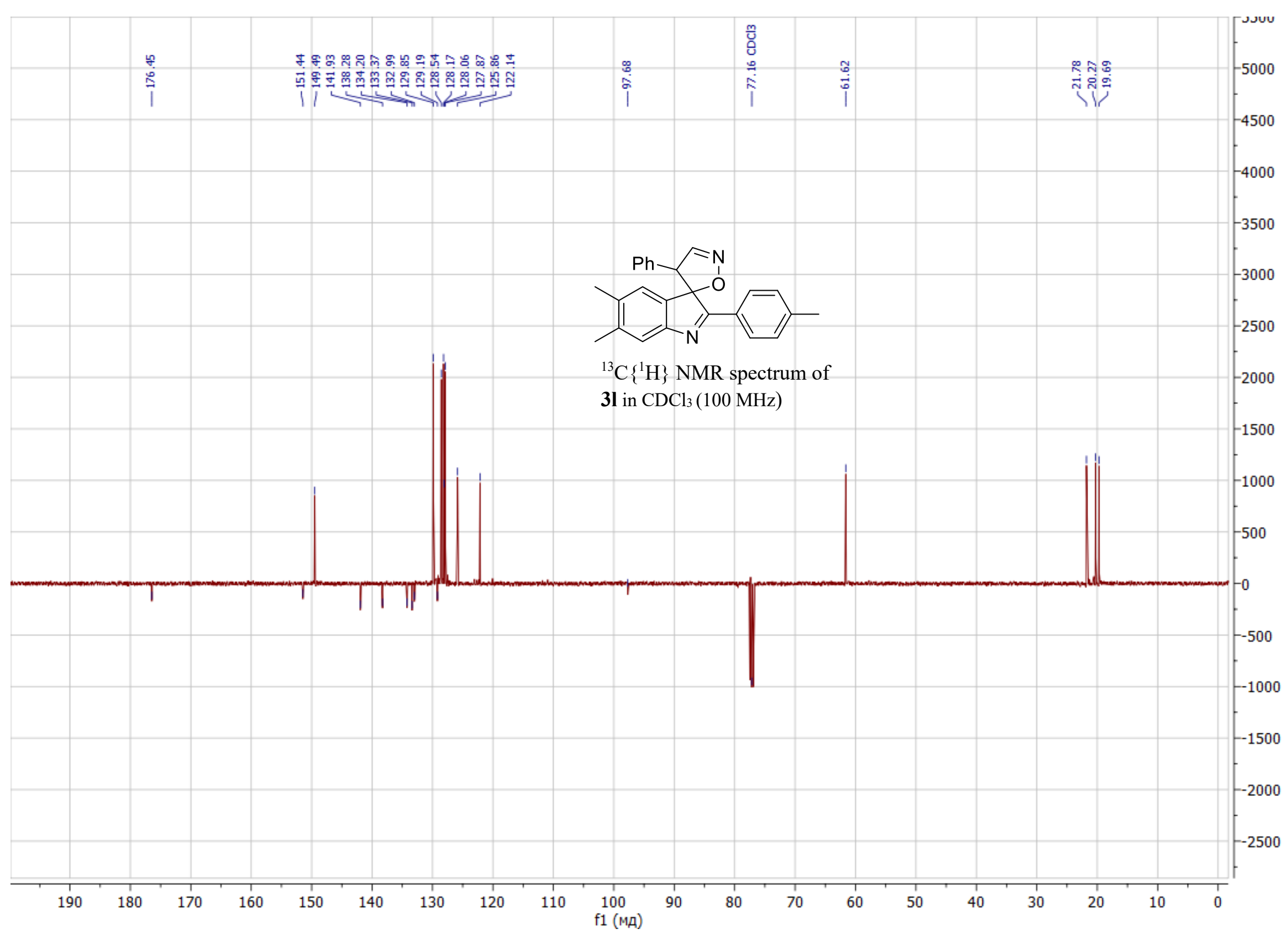




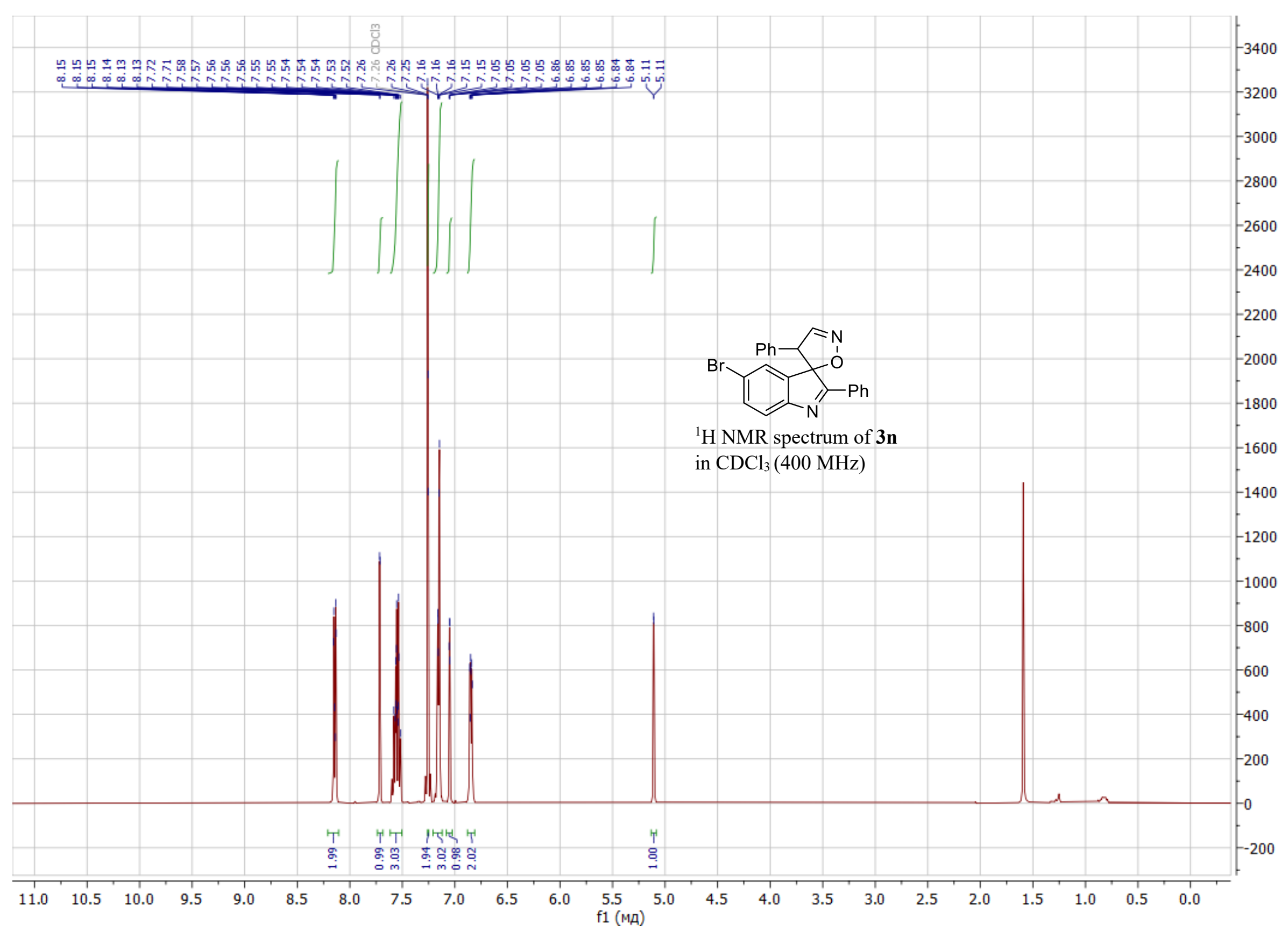




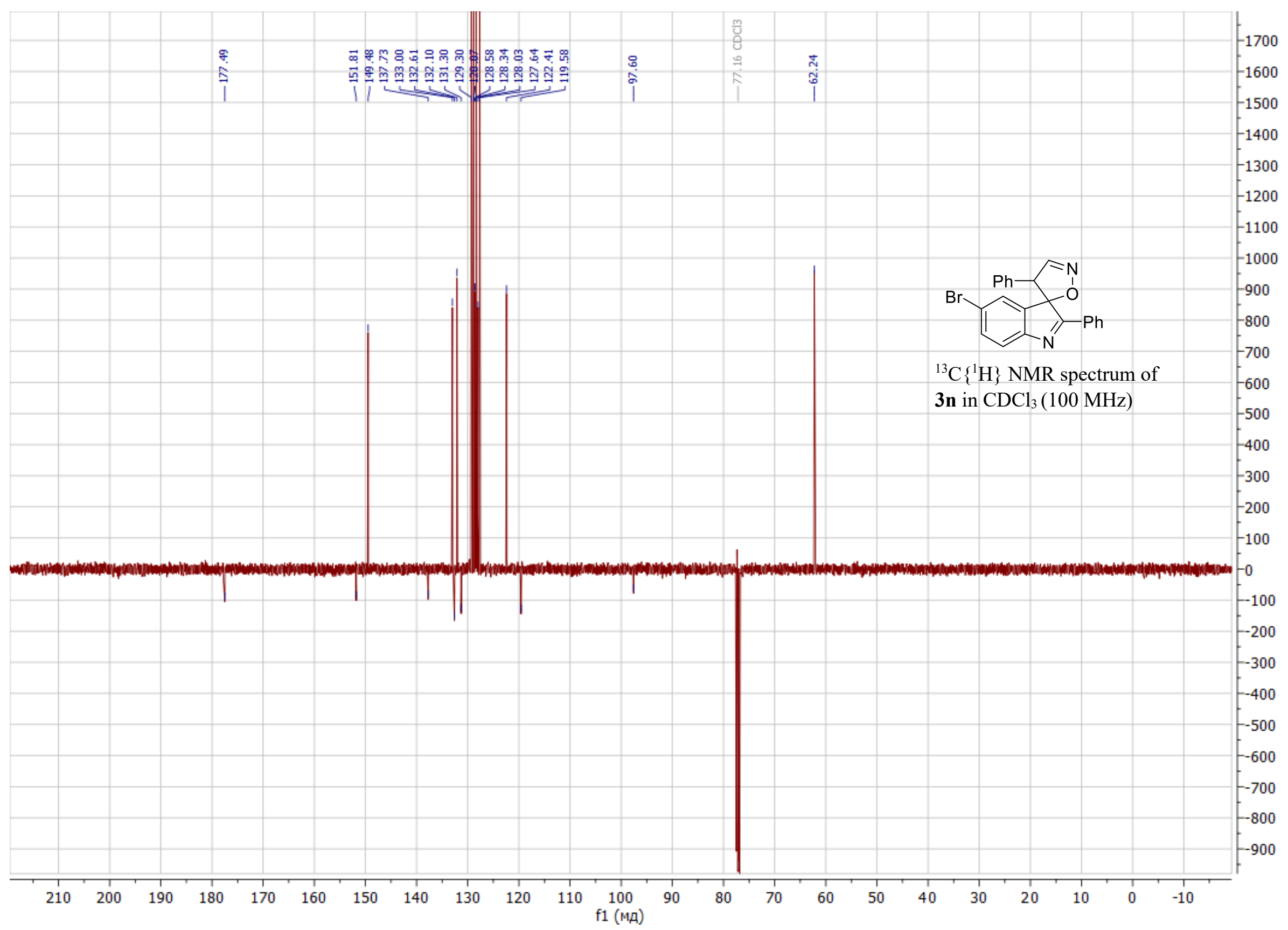




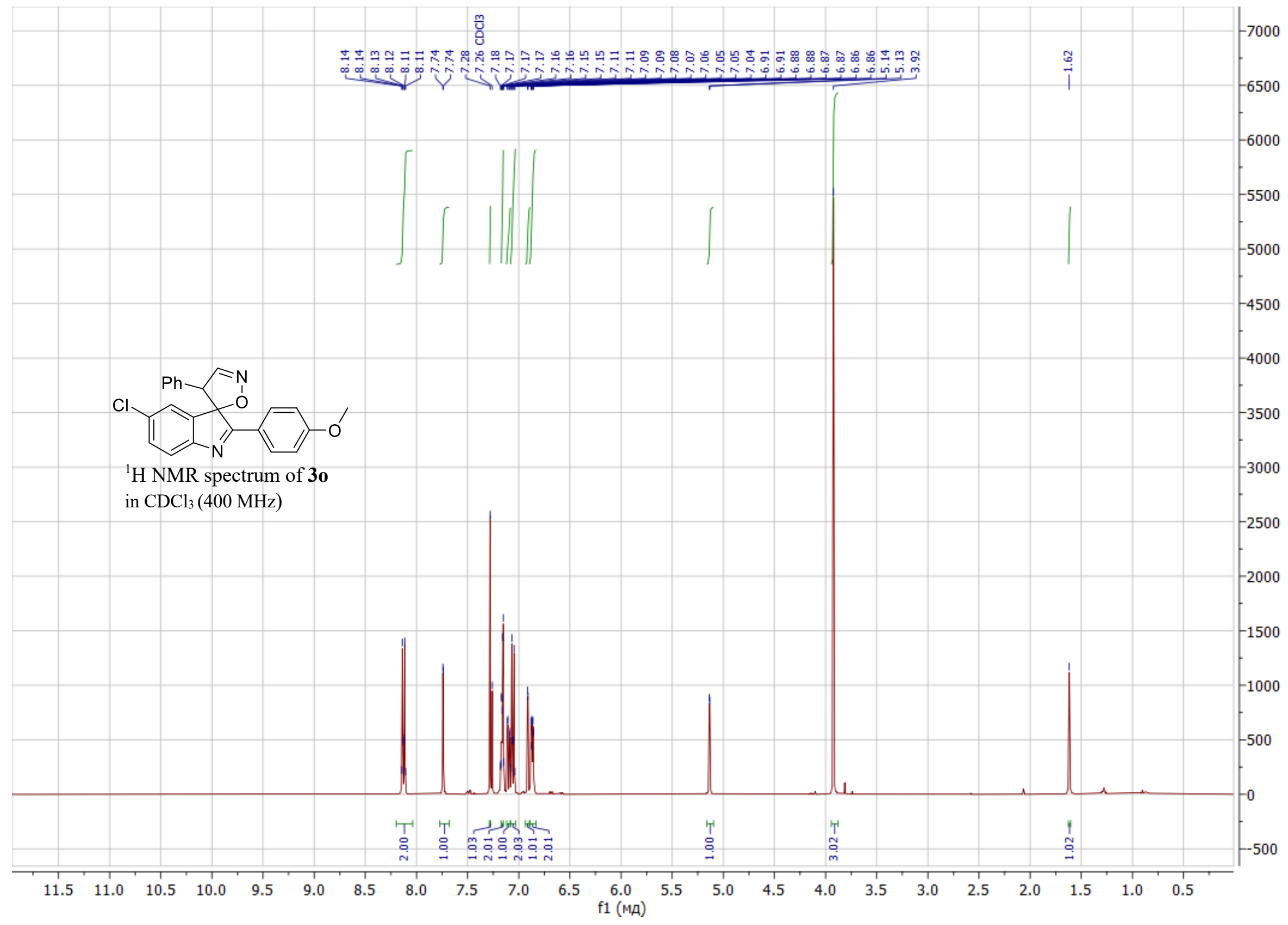




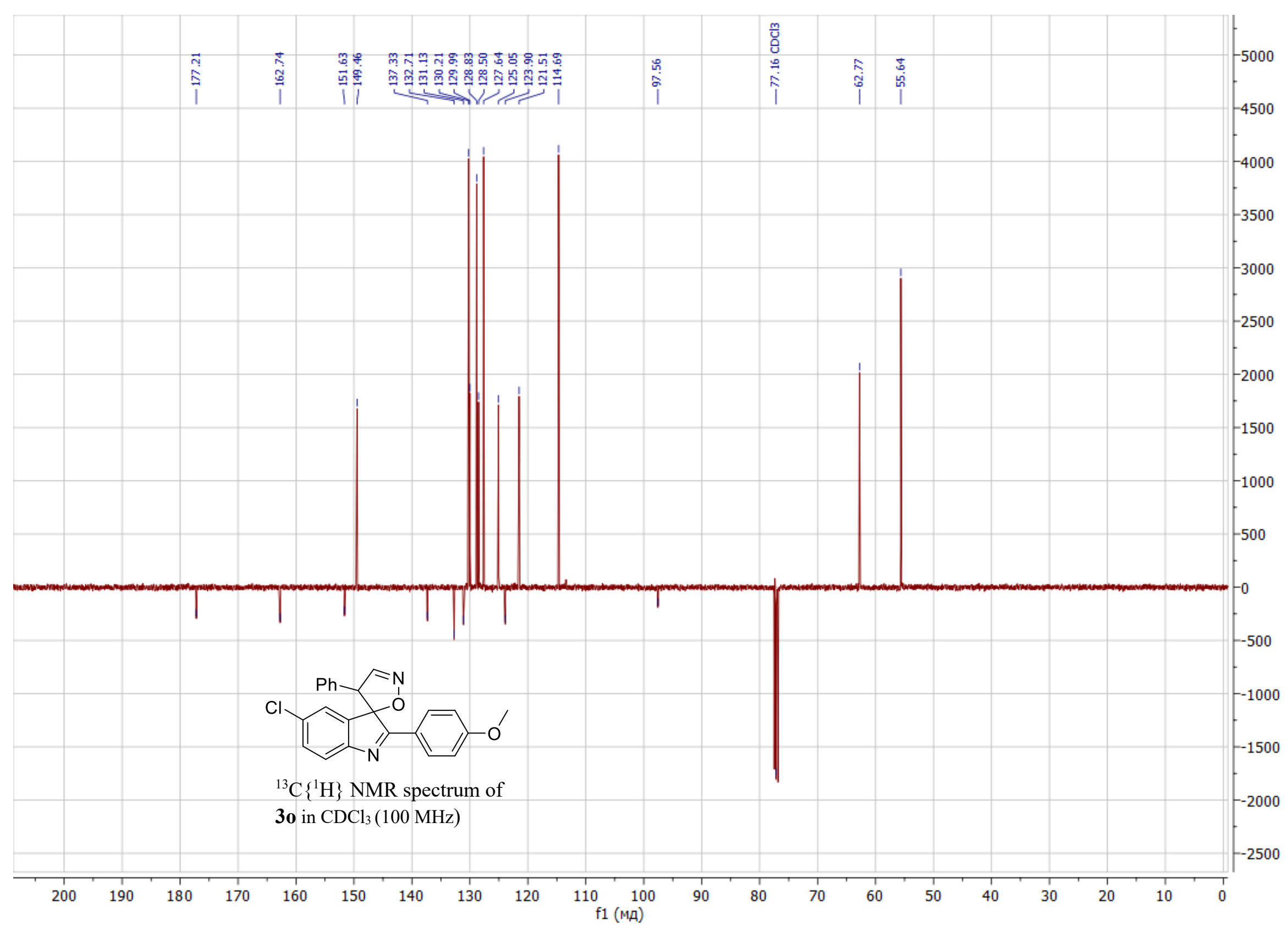


${ }^{1} \mathrm{H}$ and ${ }^{13} \mathrm{C}$ Spectral Charts for 2-(3-oxoindolin-2-yl)acetonitriles 4

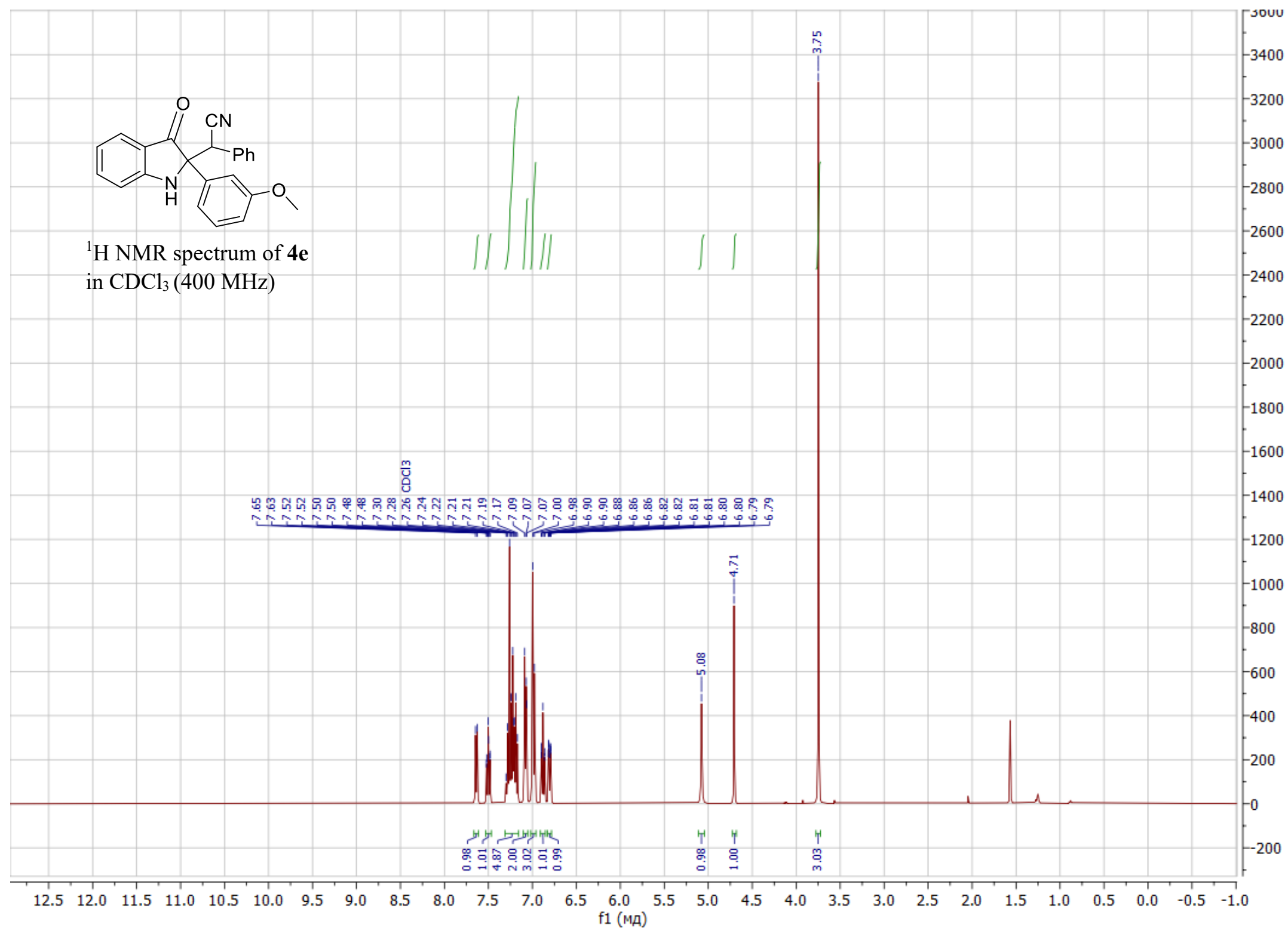




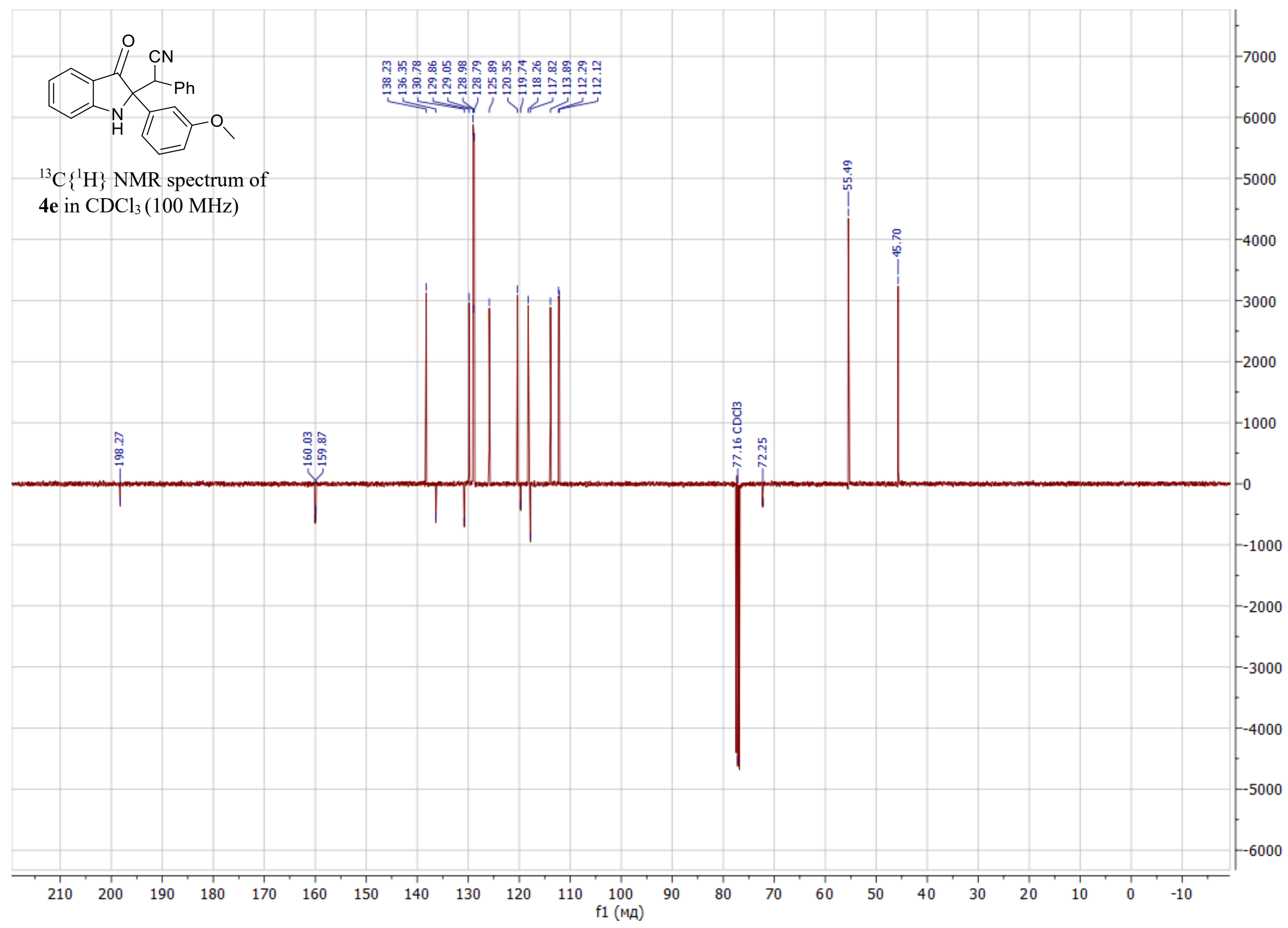




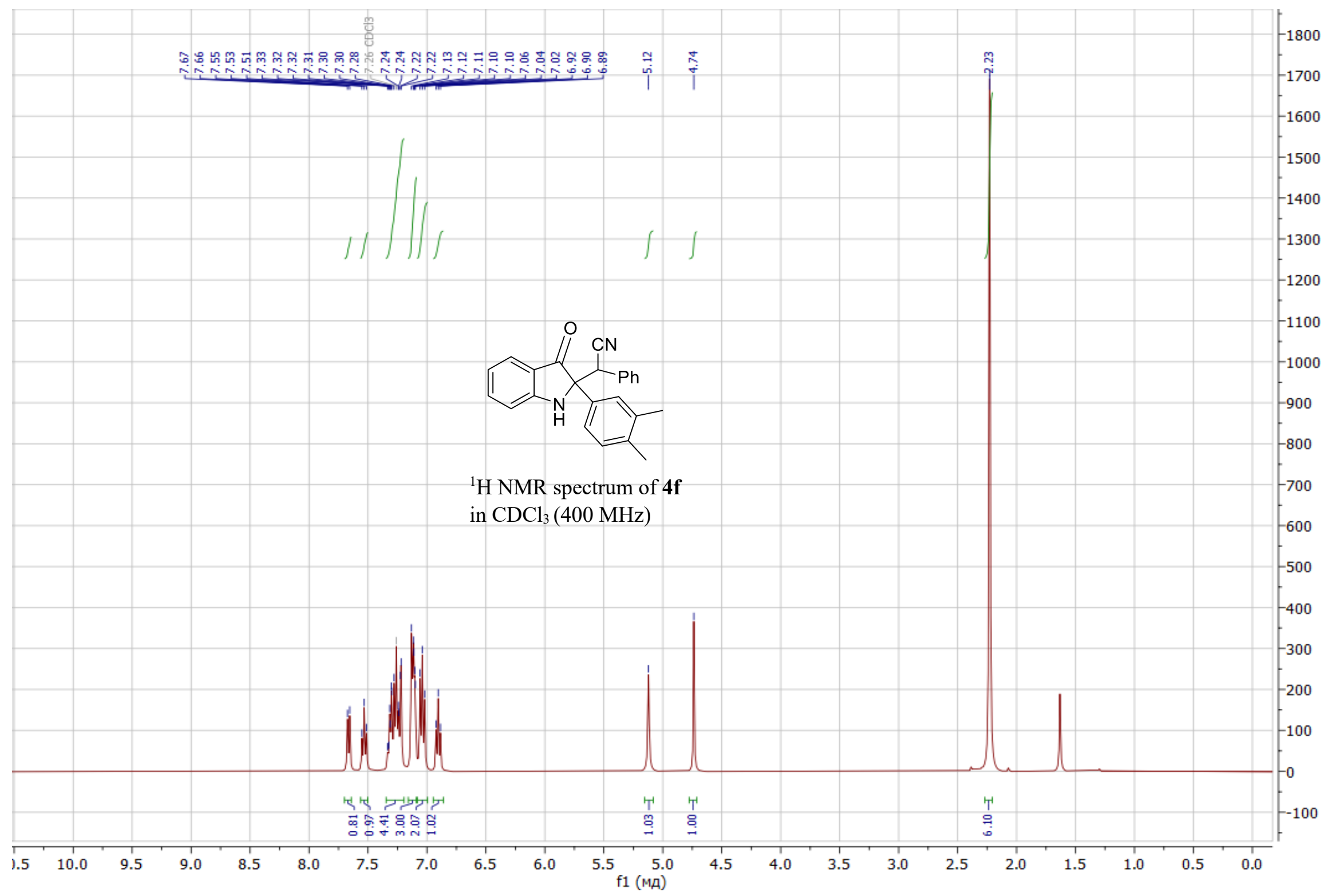




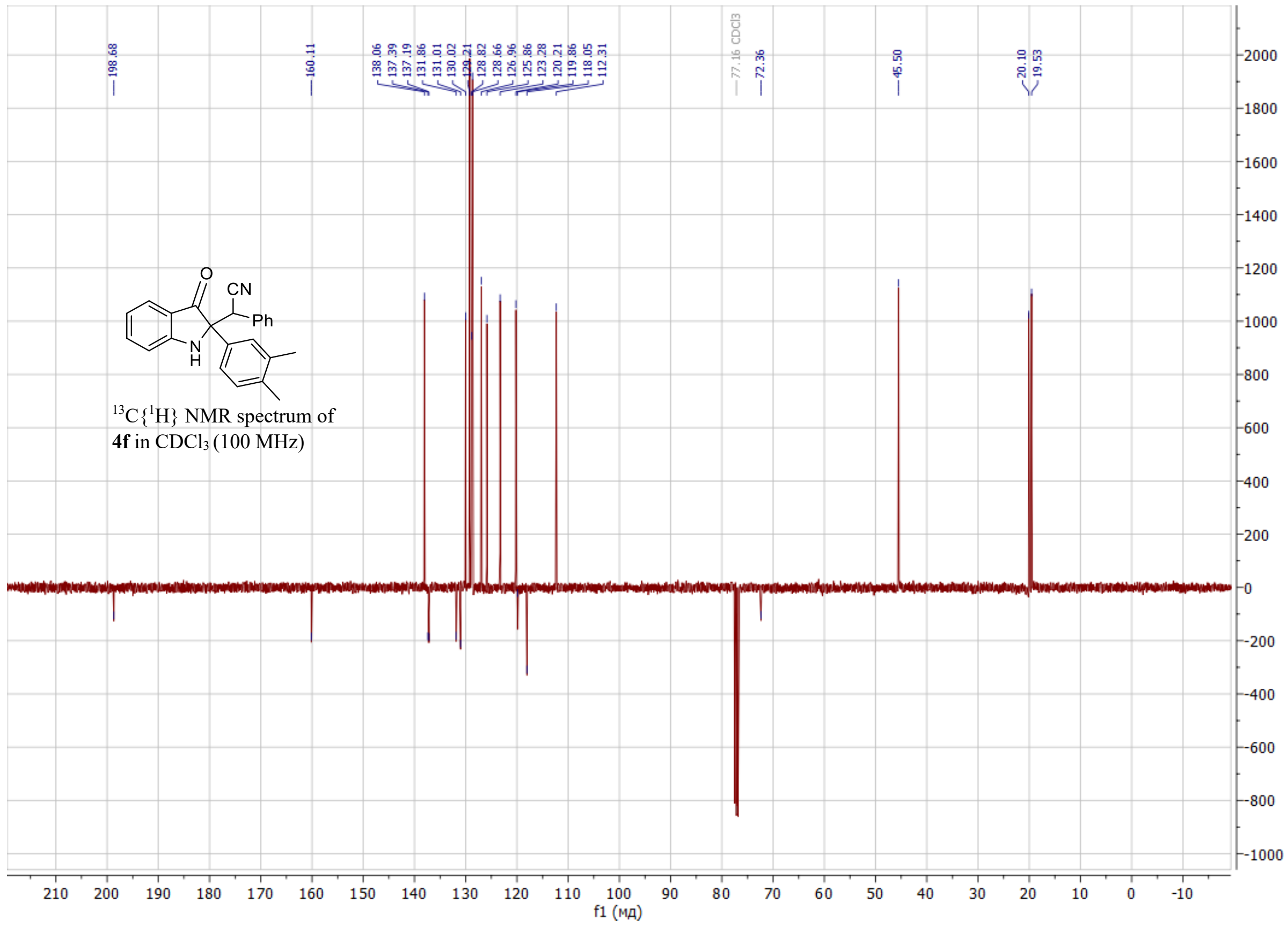

S21 


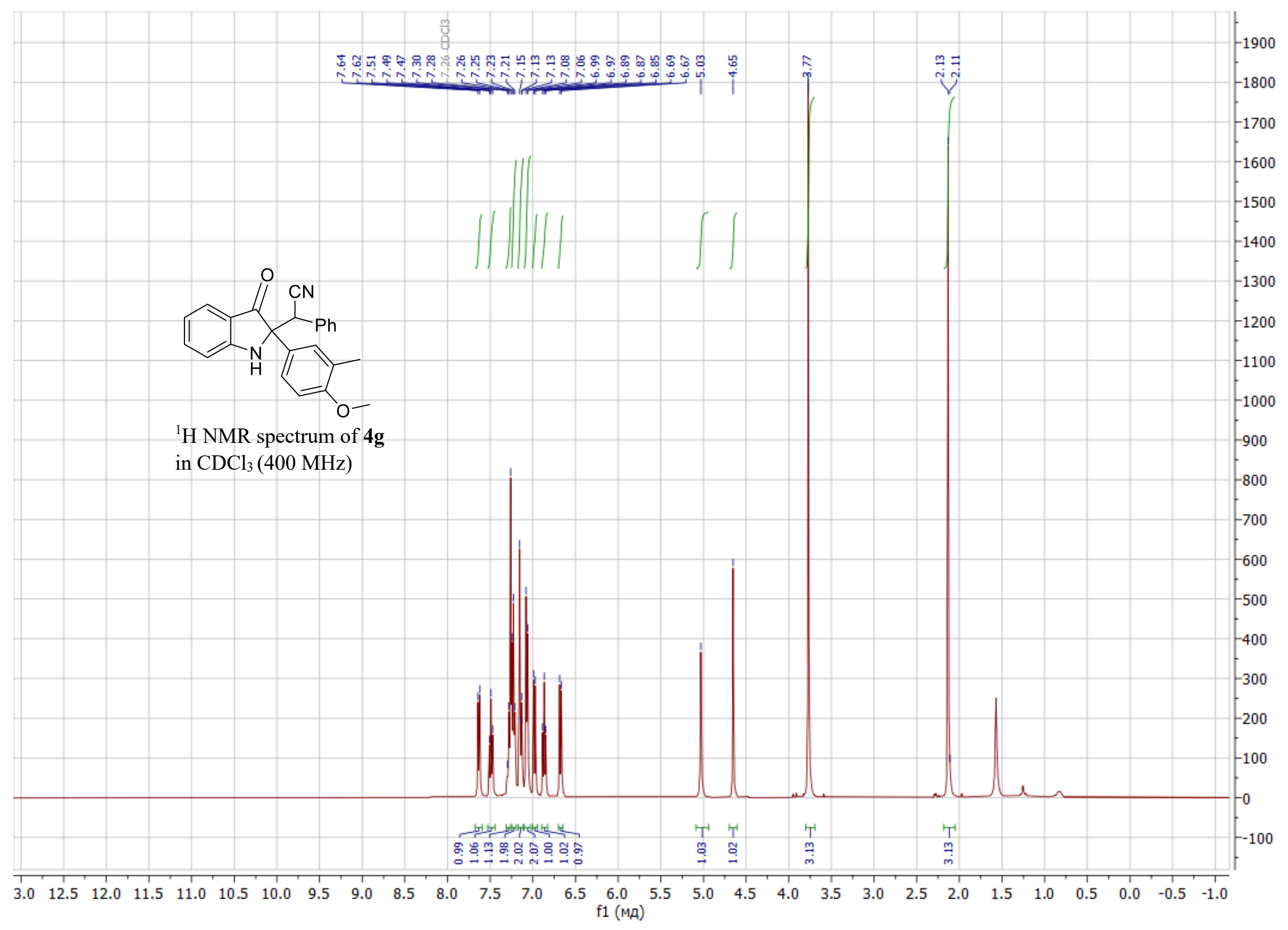




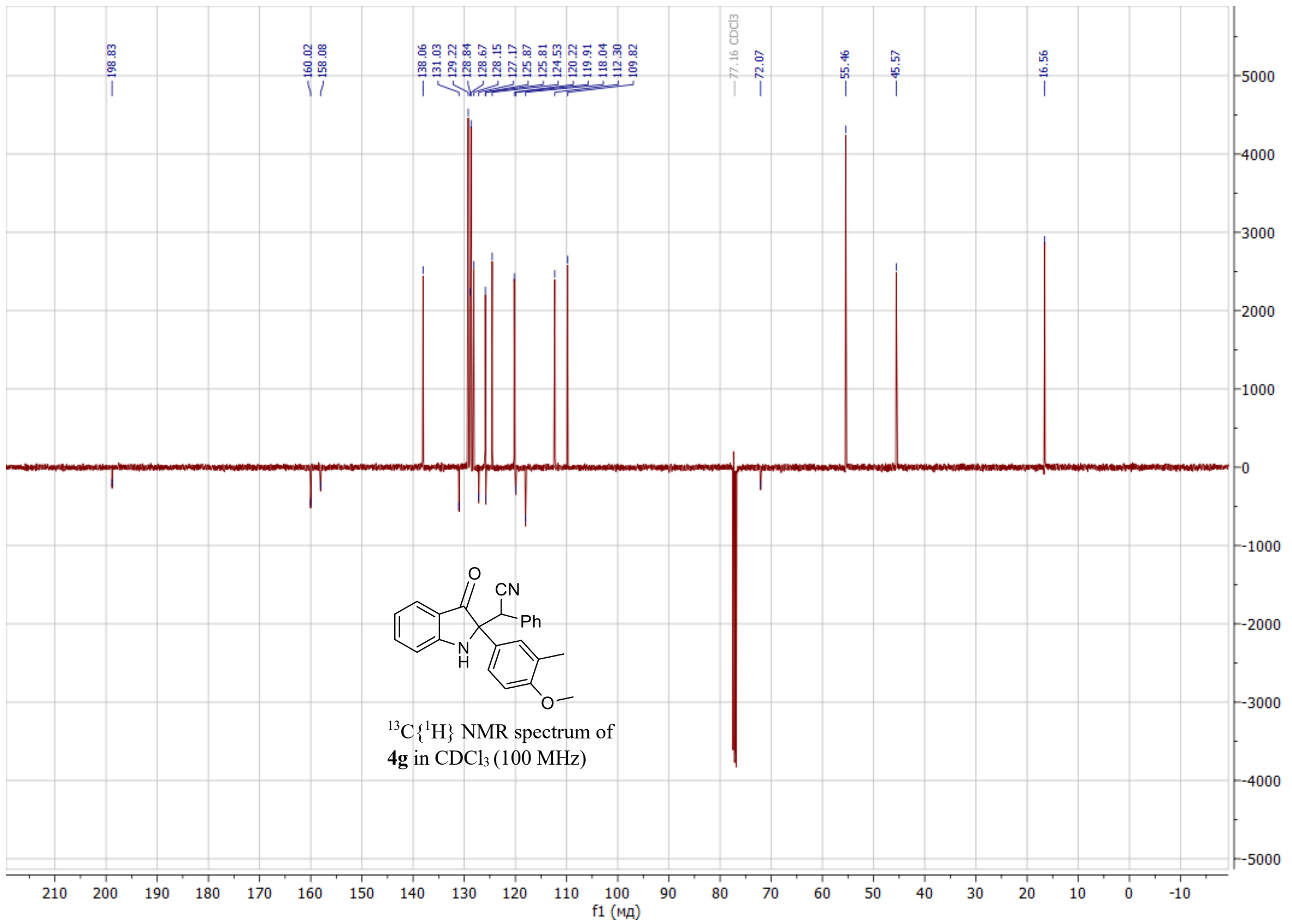

S23 


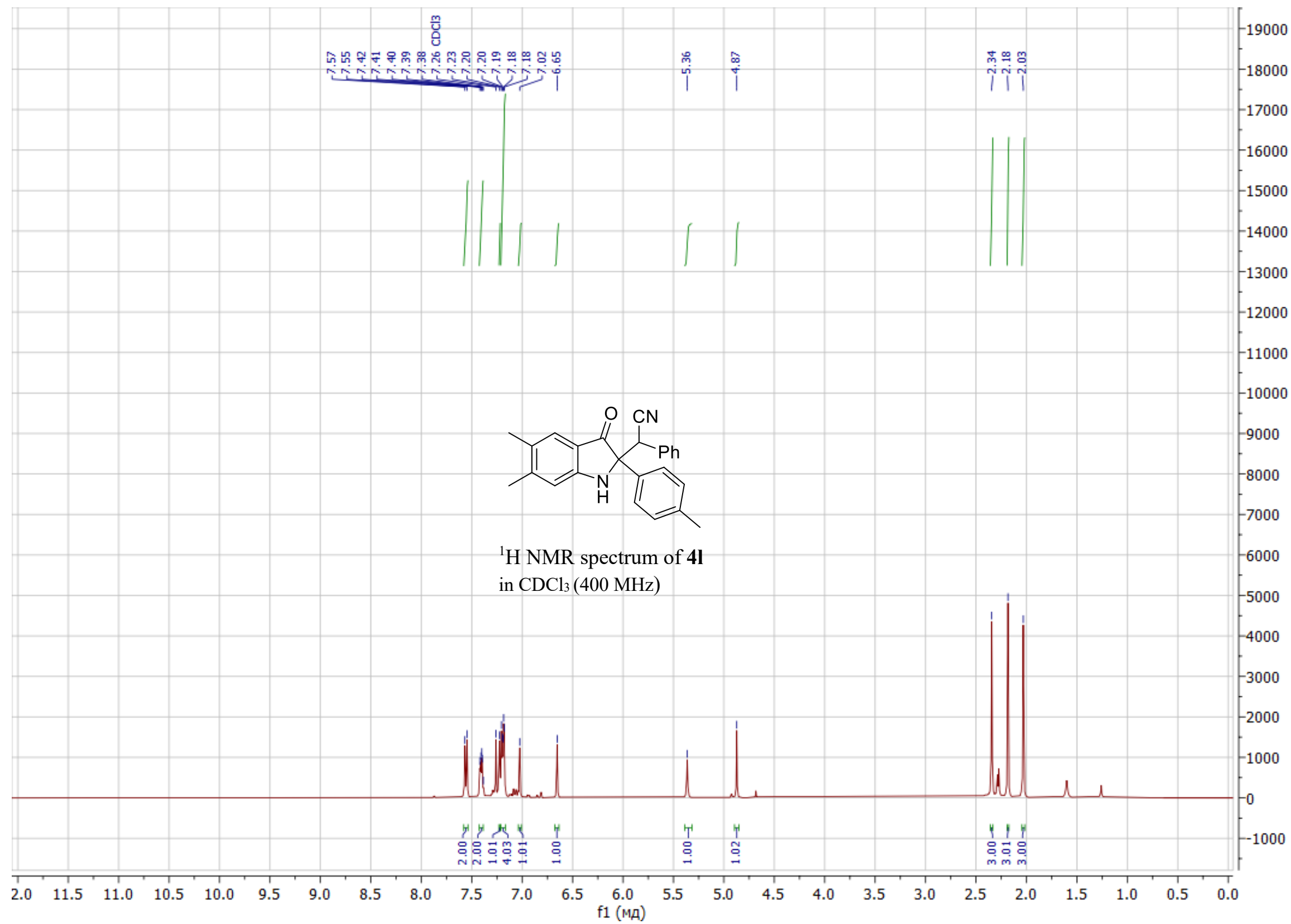




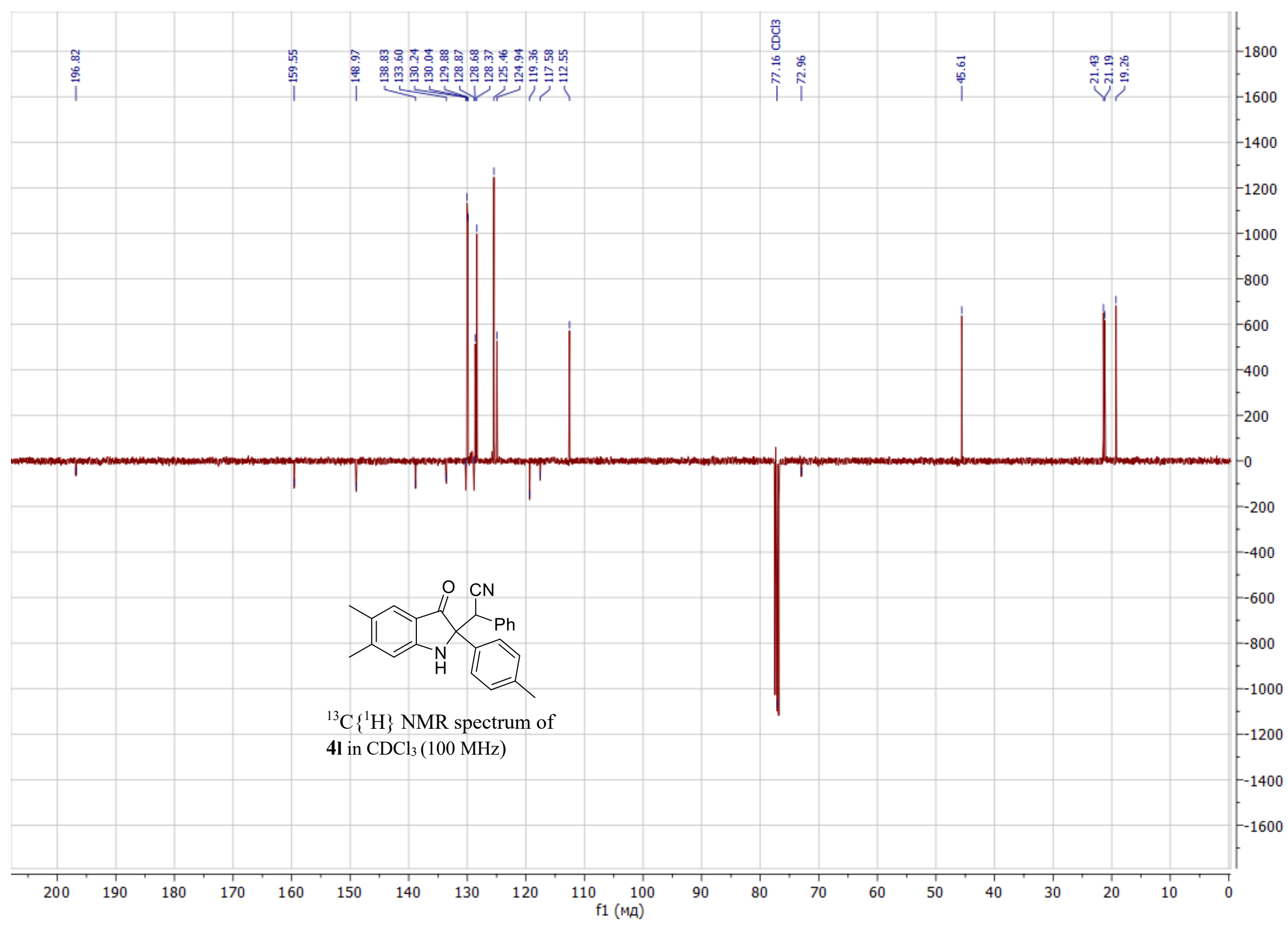




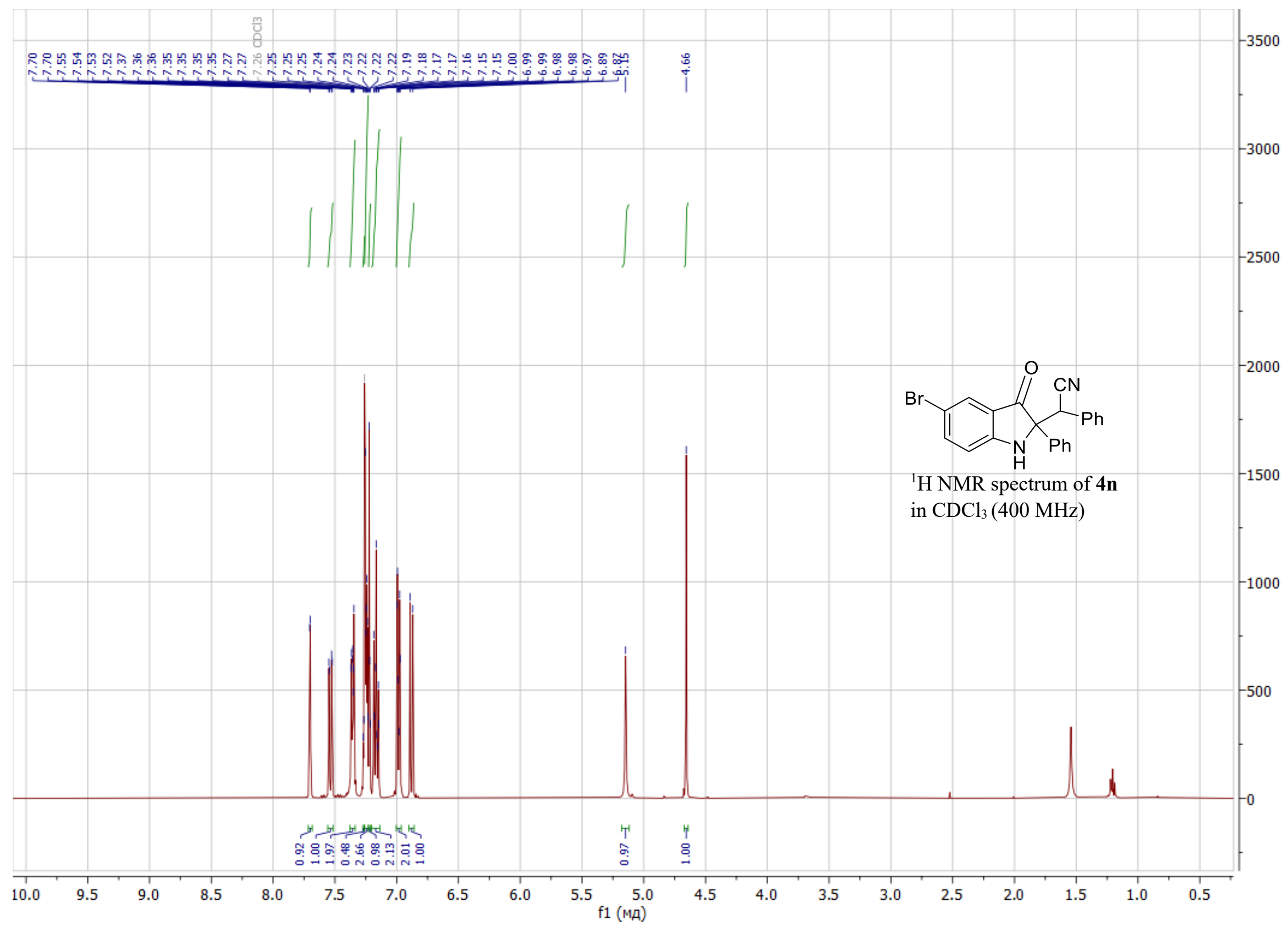




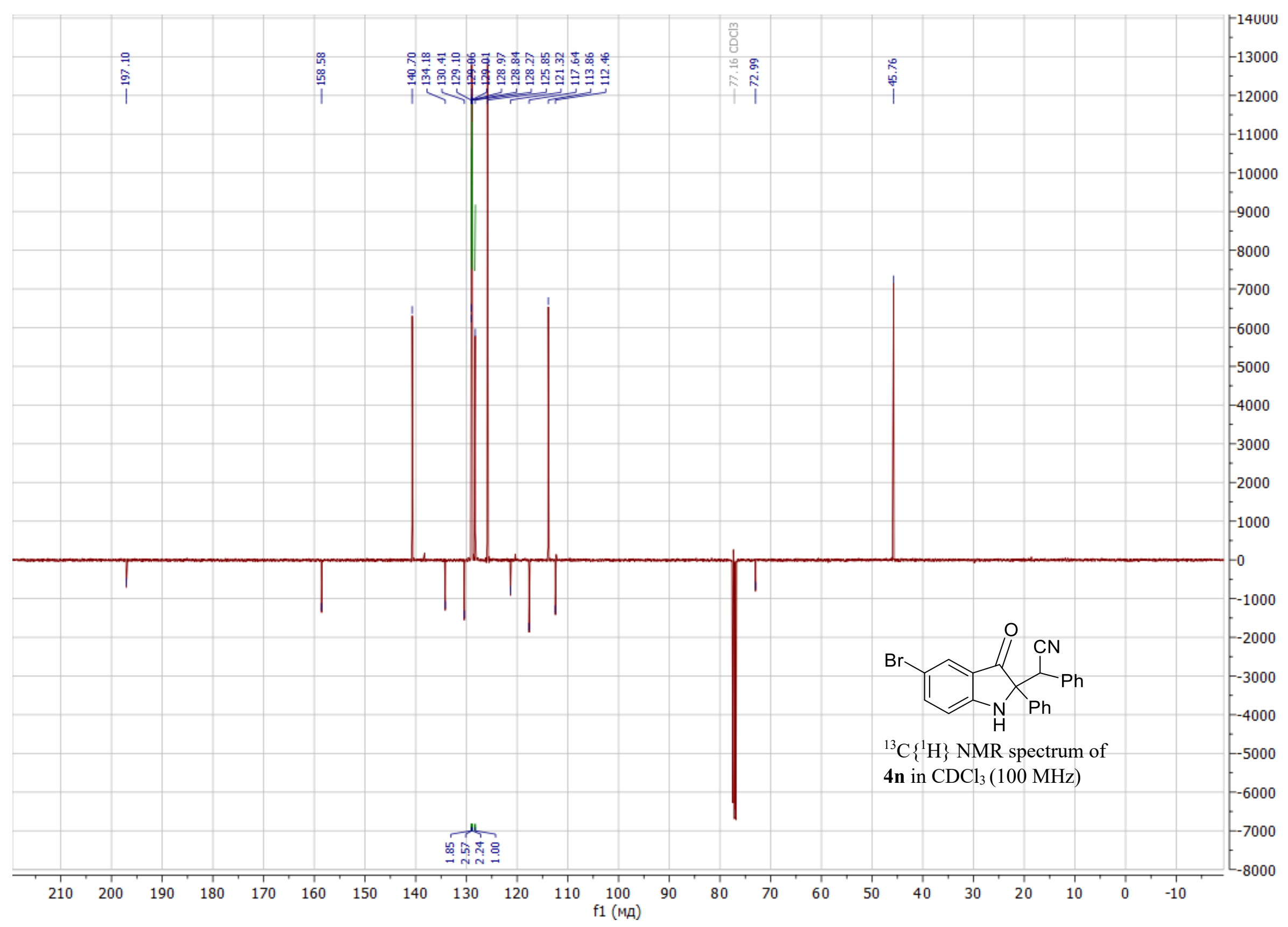




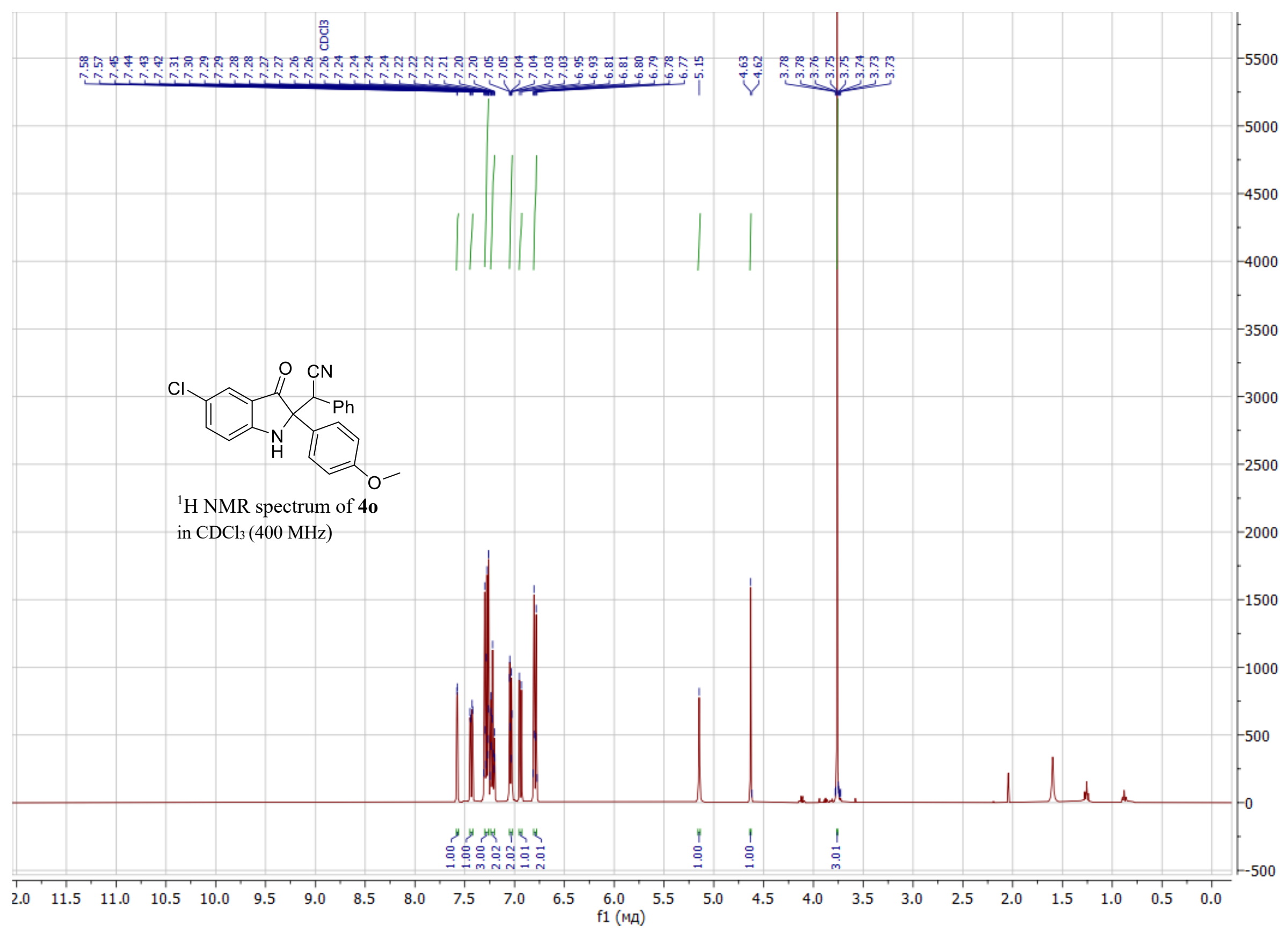




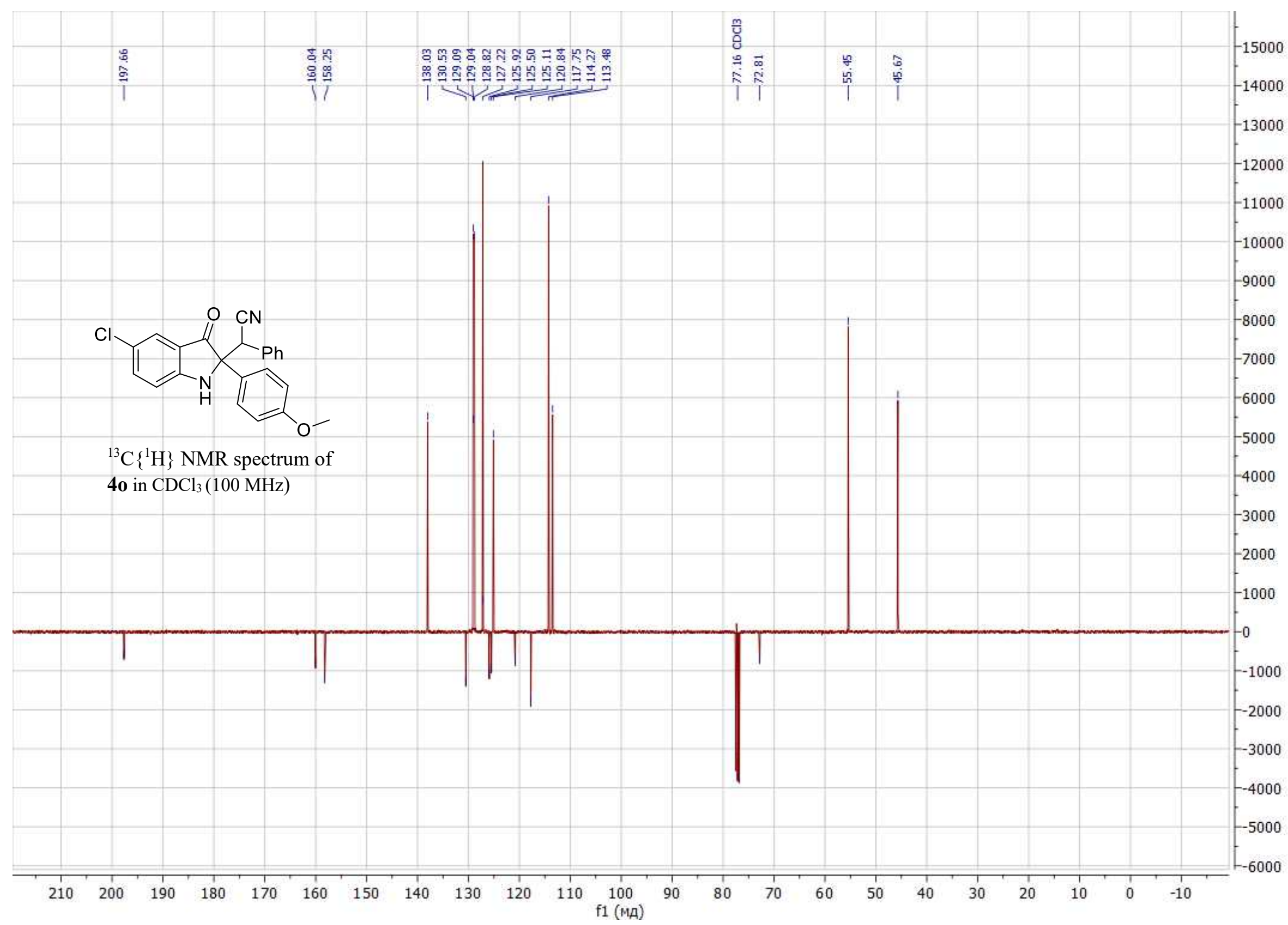




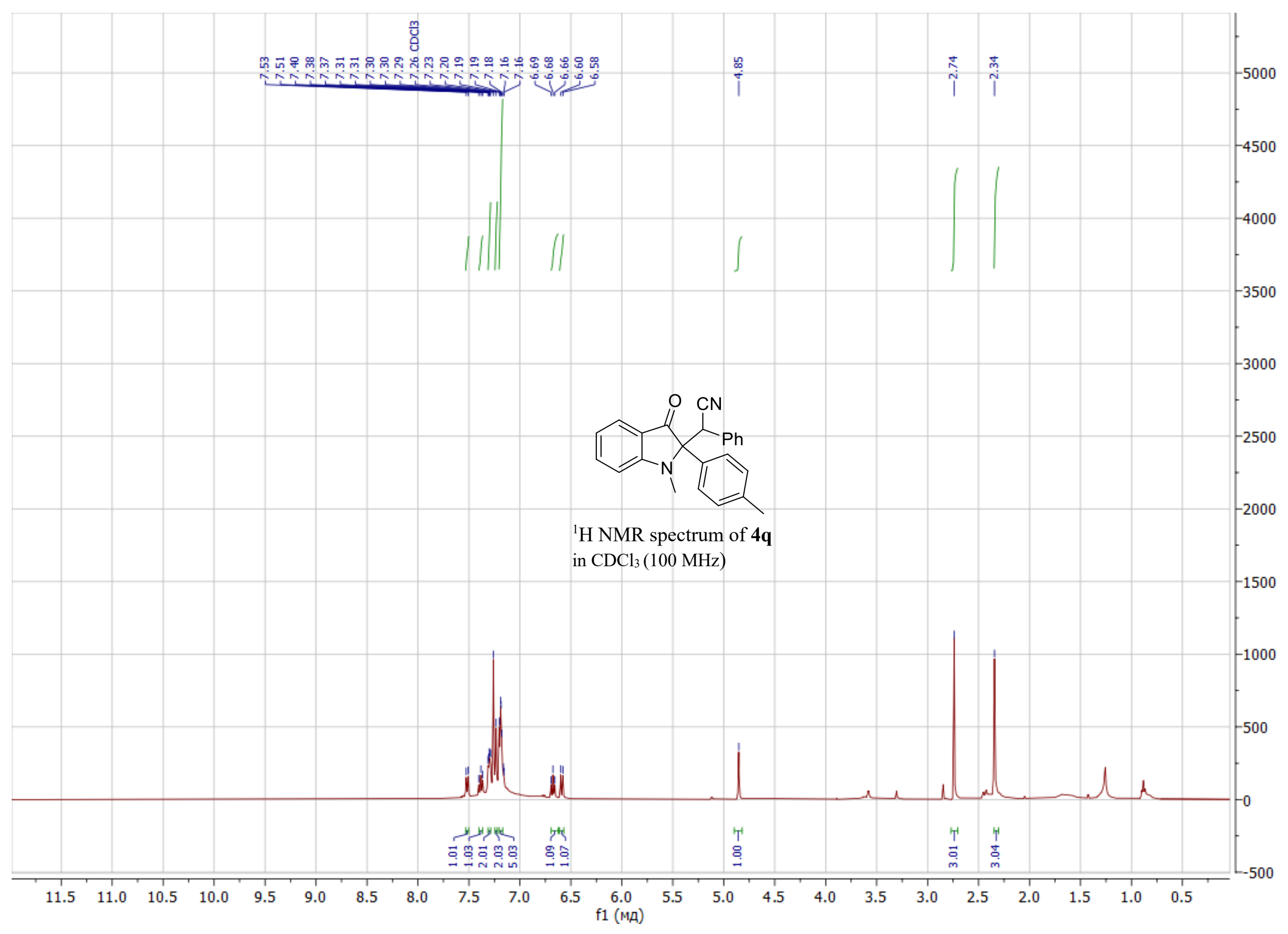




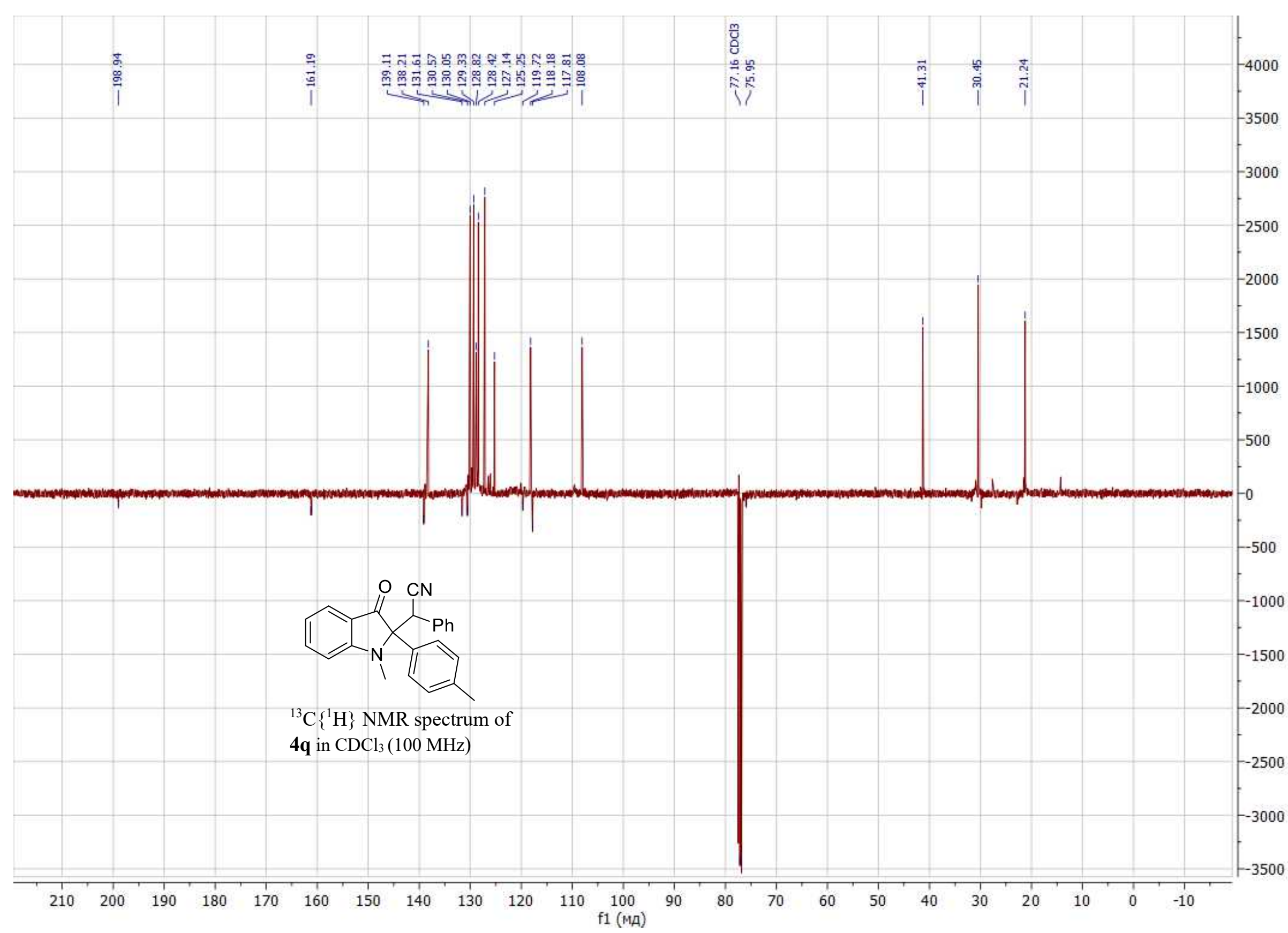




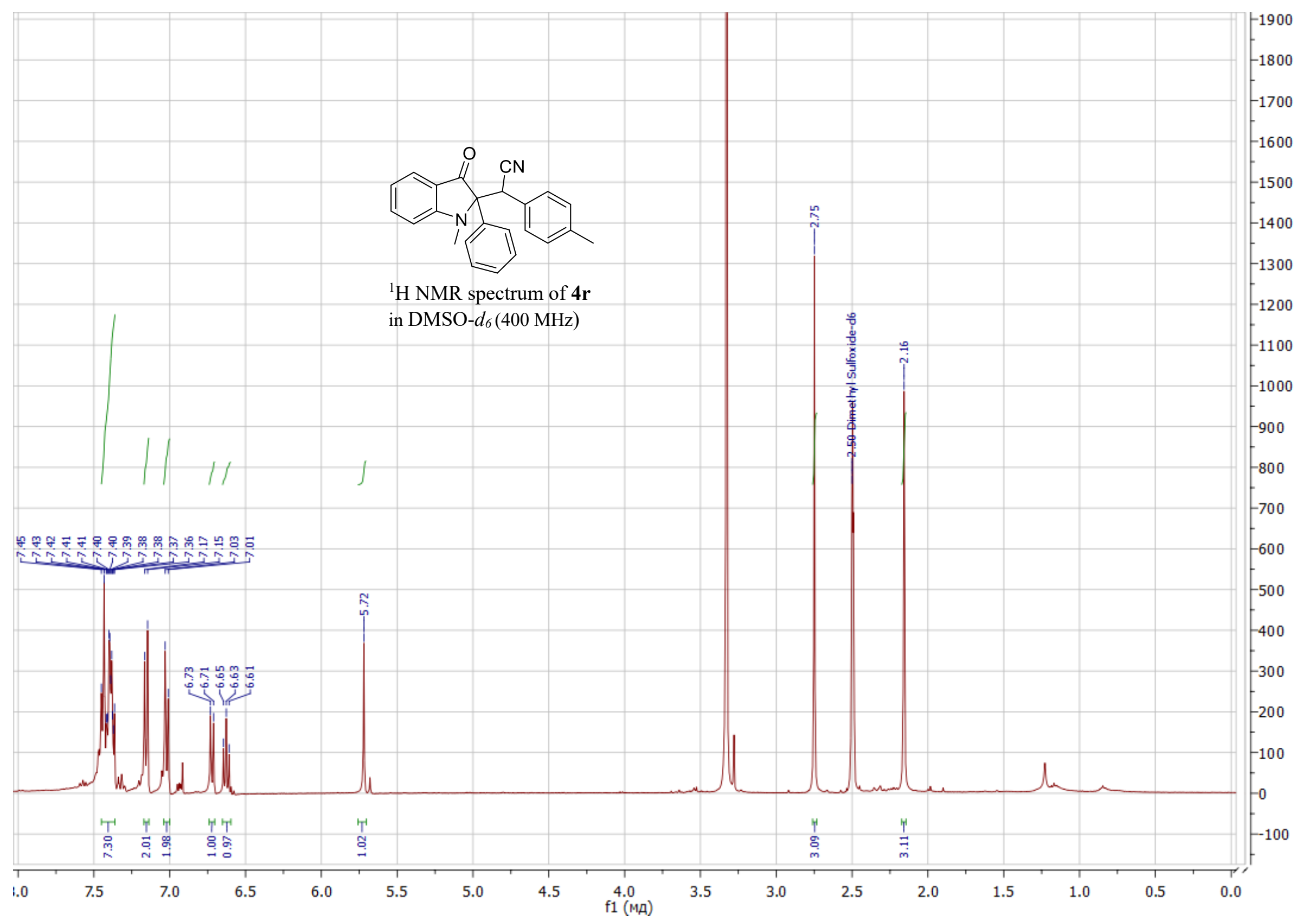




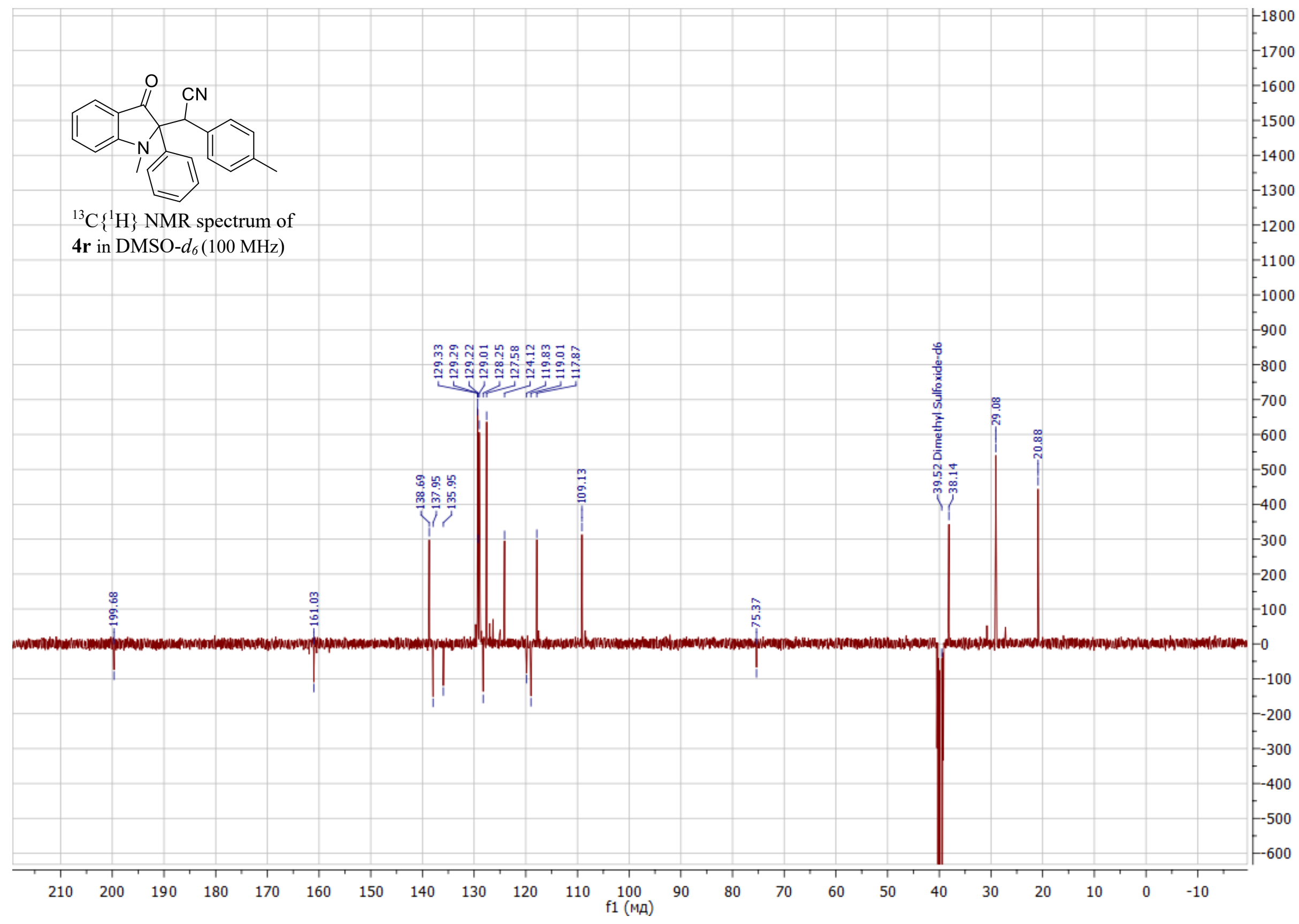




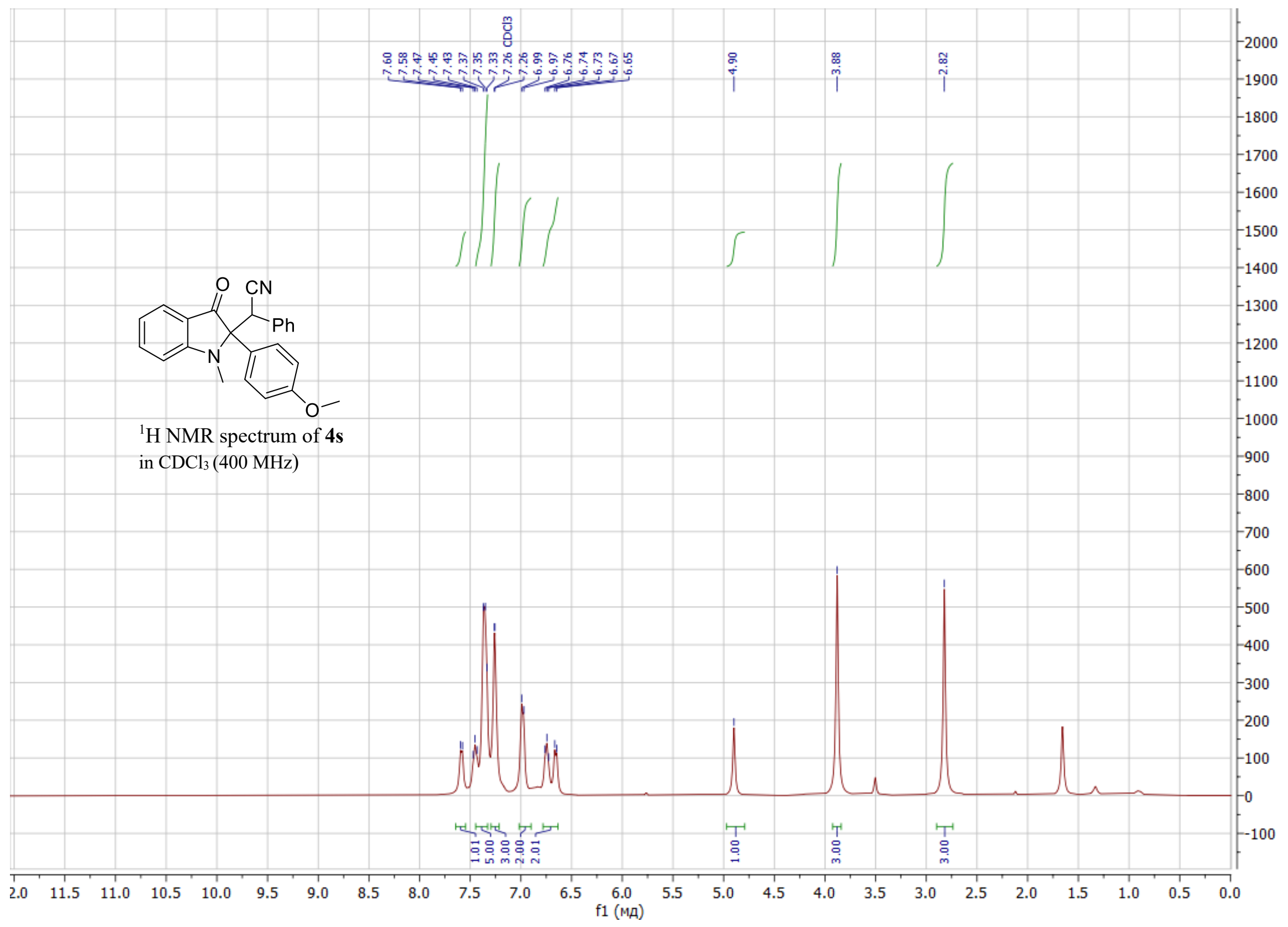




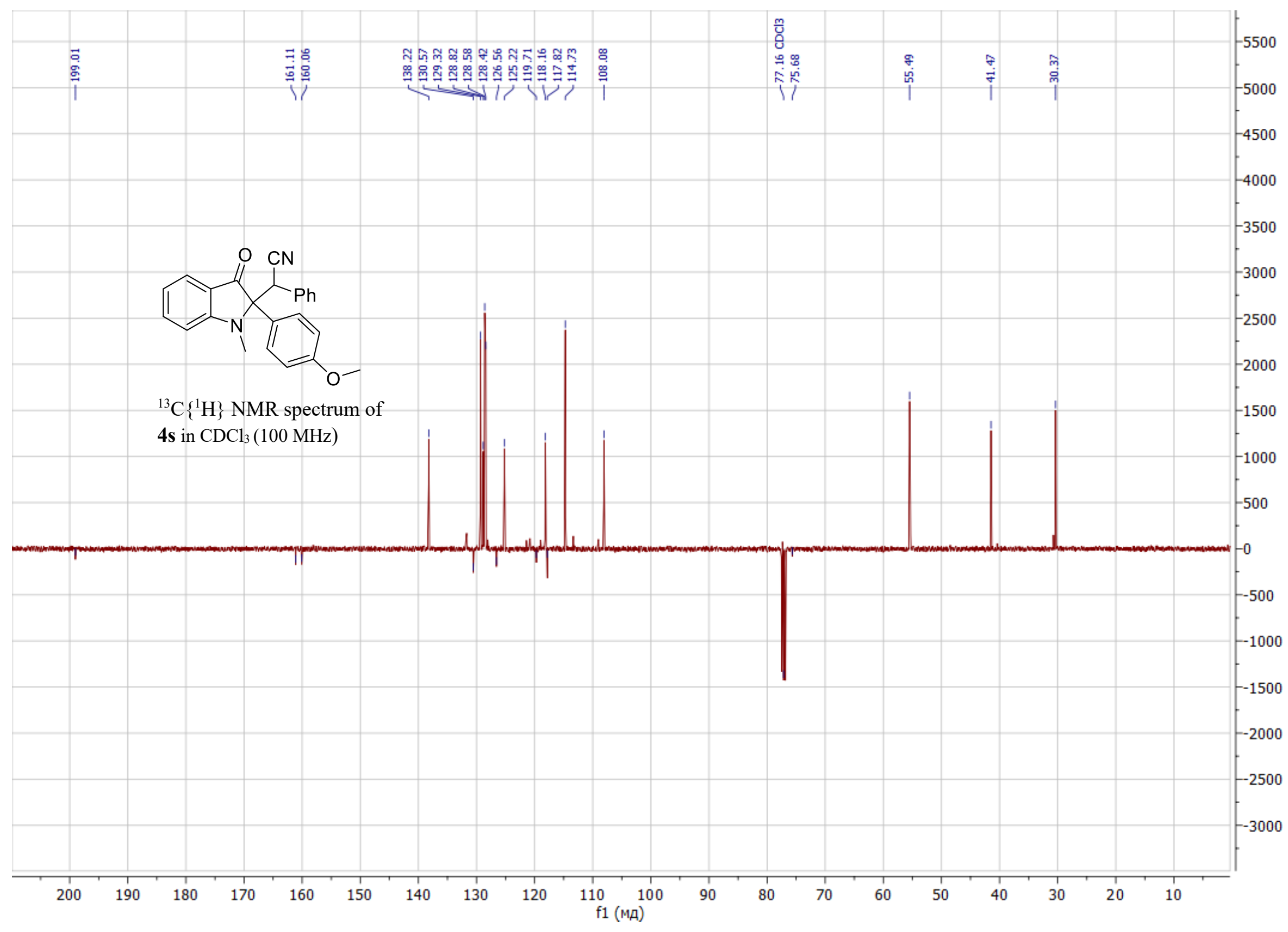




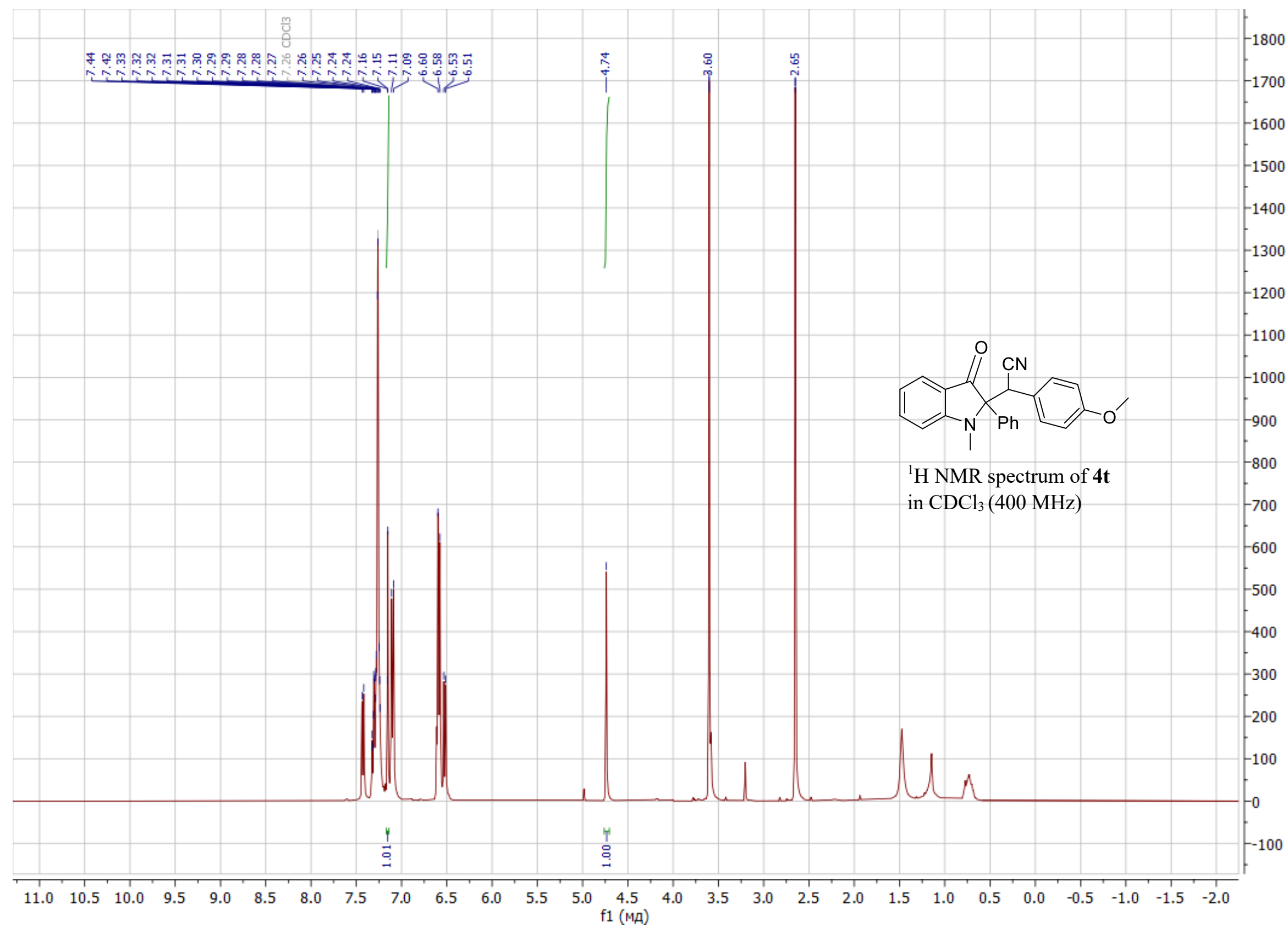




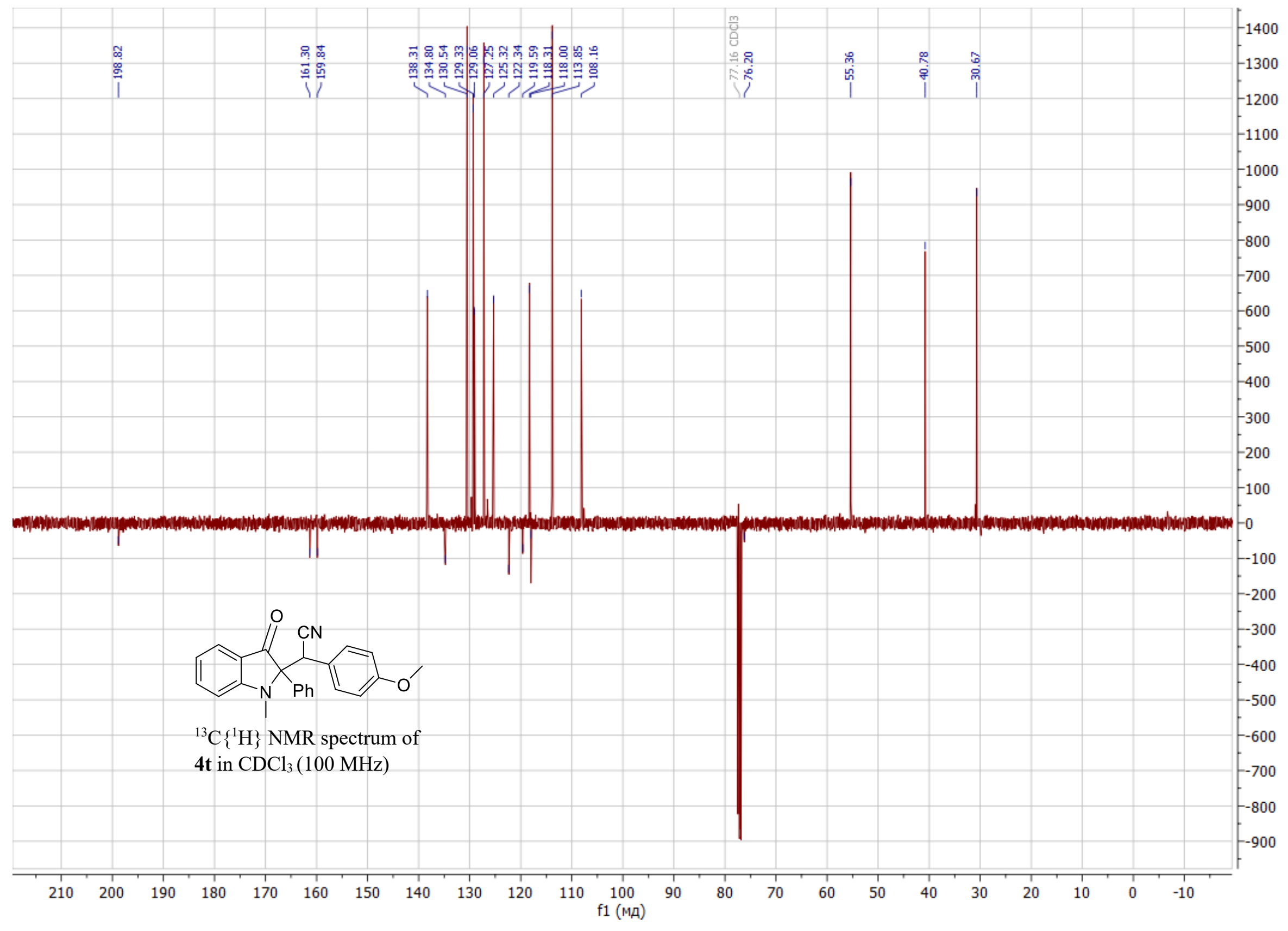




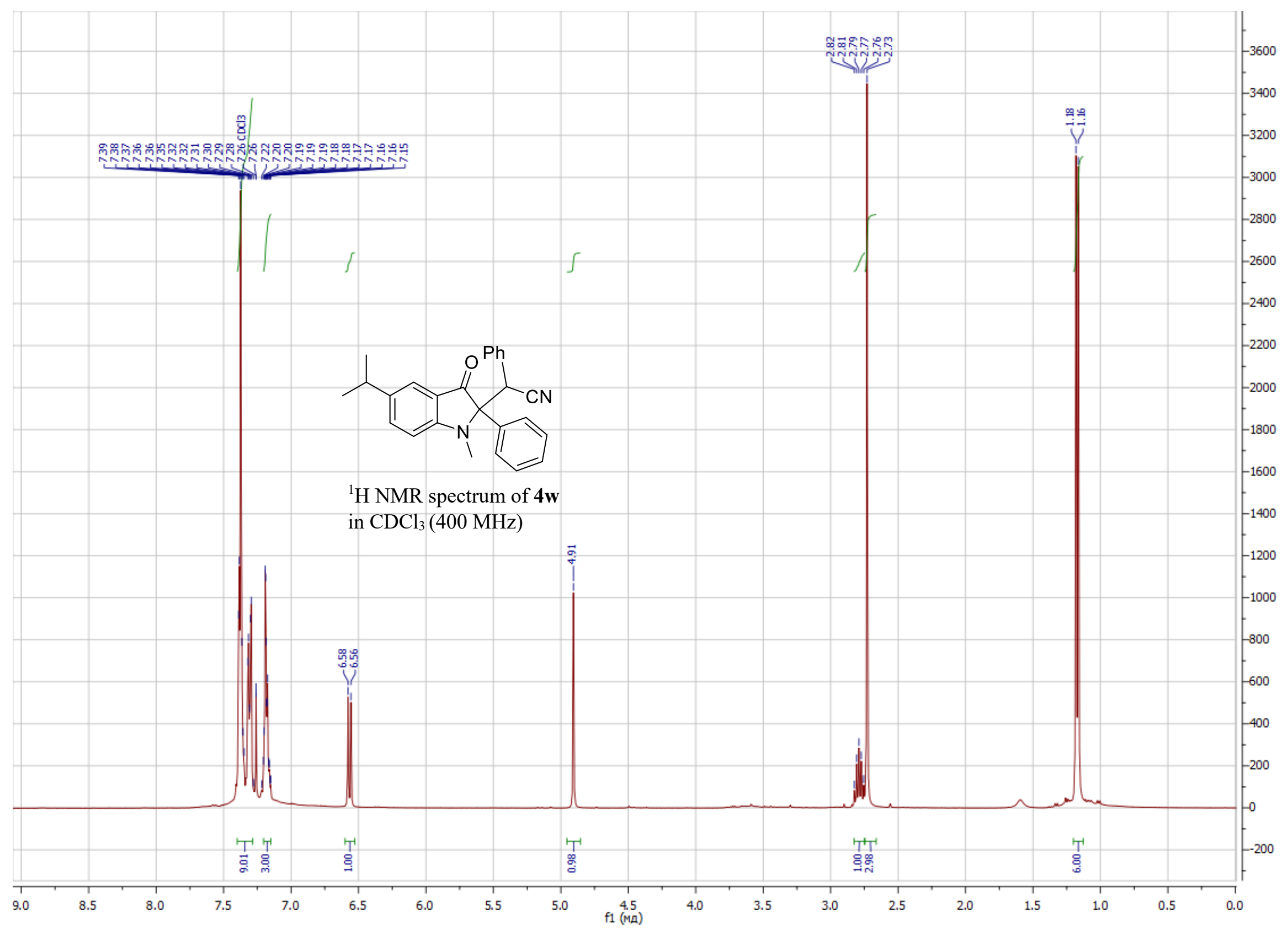




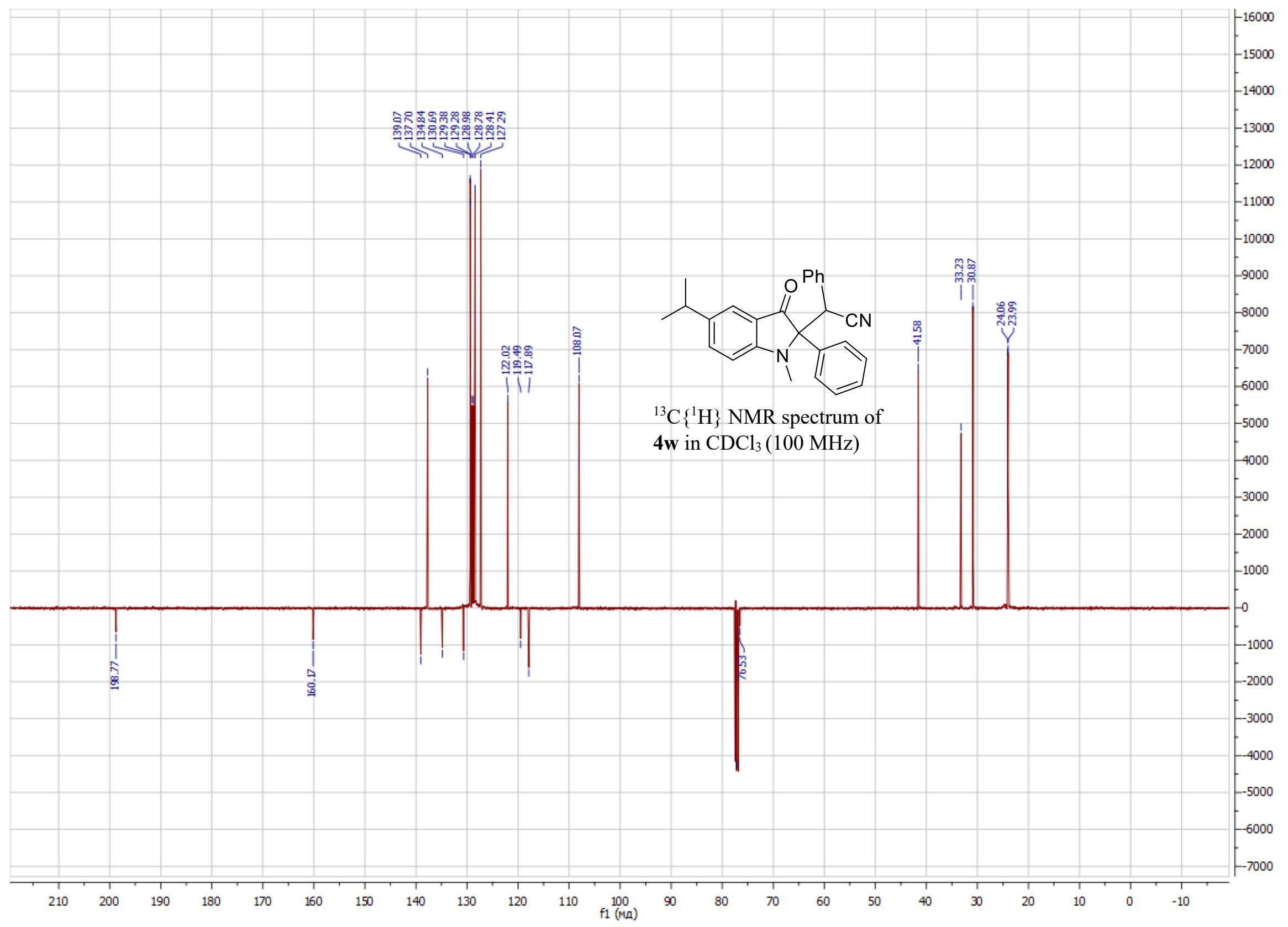




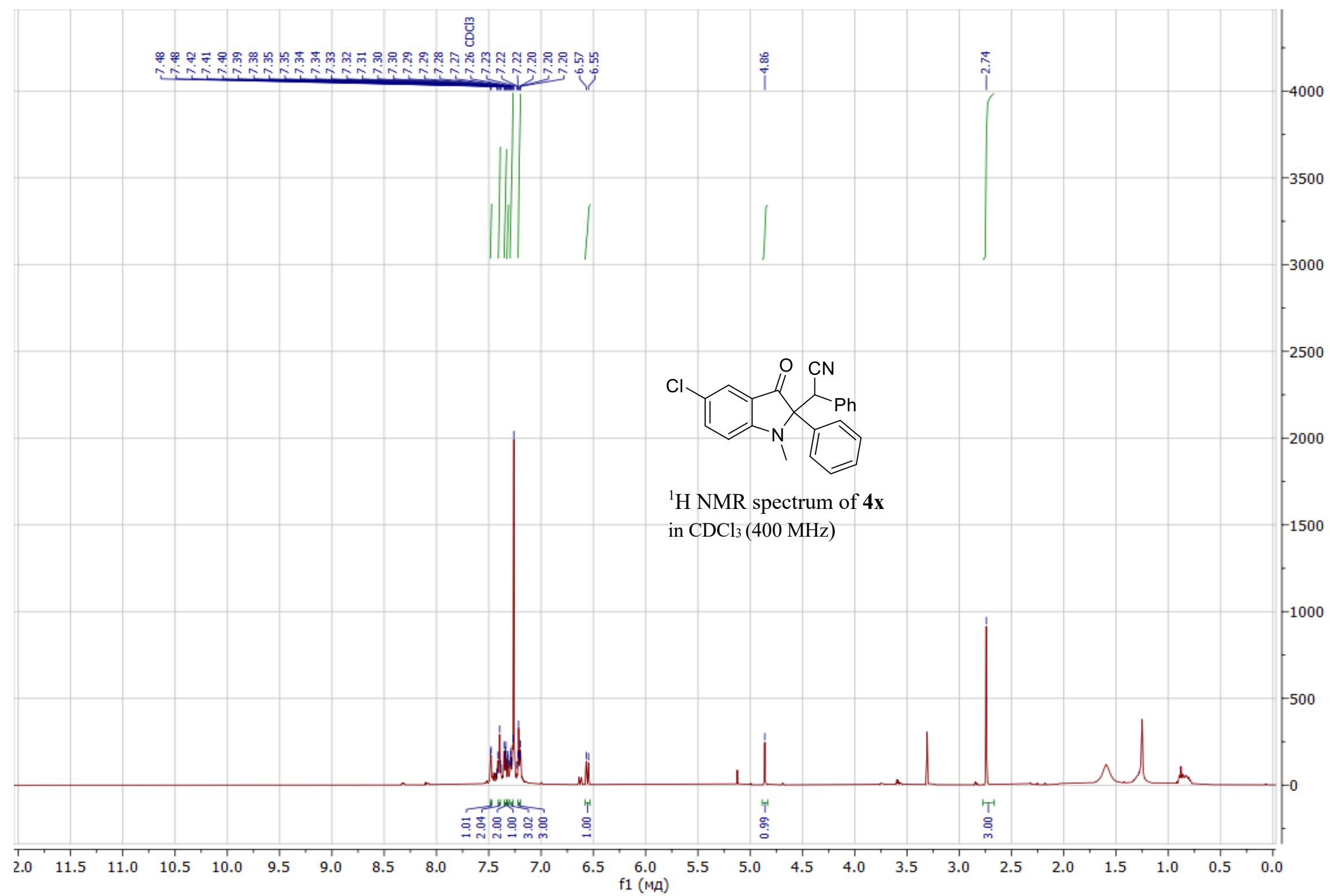




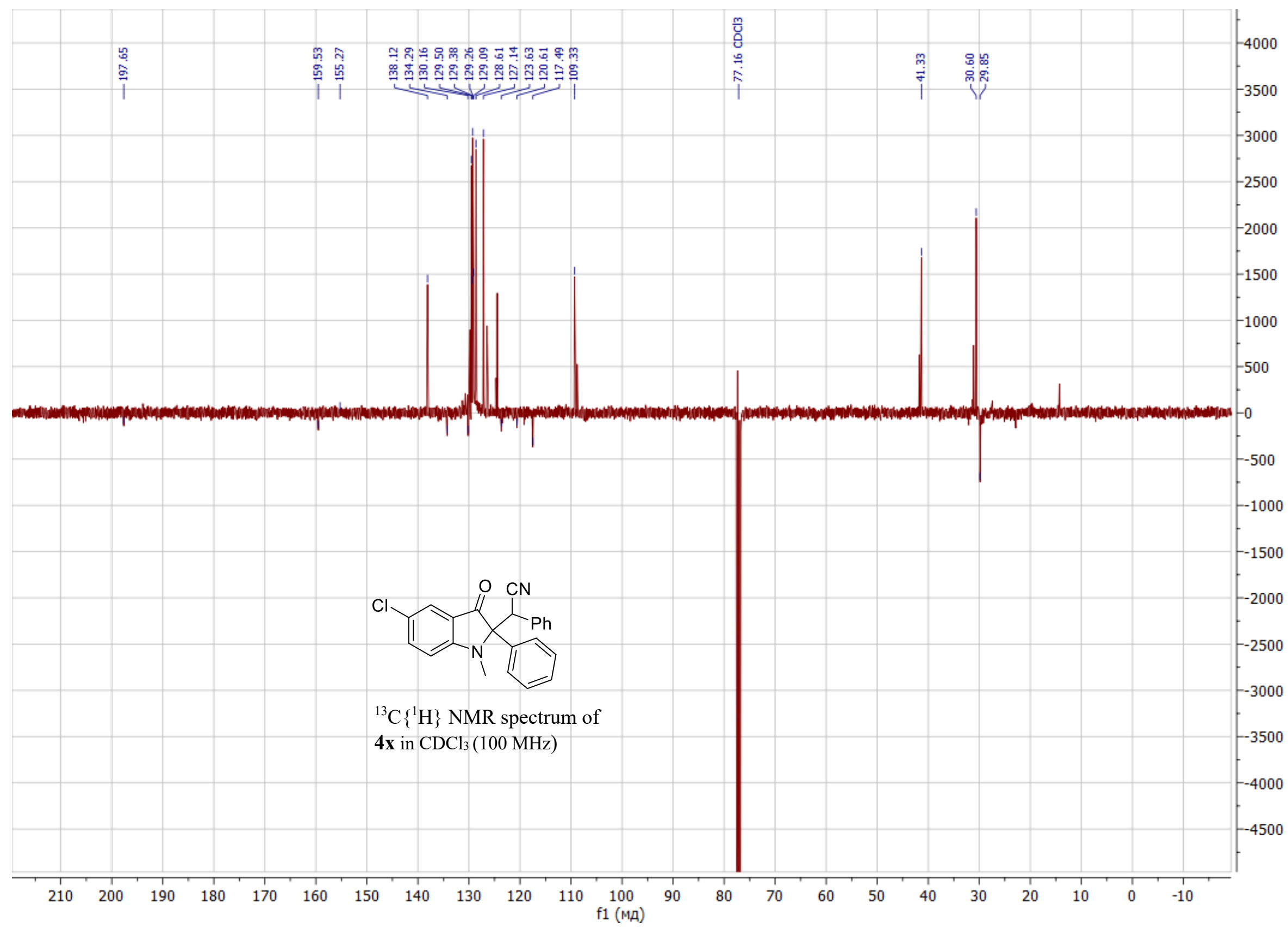


${ }^{1} \mathrm{H}$ and ${ }^{13} \mathrm{C}$ Spectral Charts of 1,3a,4,8b-tetrahydropyrrolo[3,2-b]indol-2(1H)-ones 5

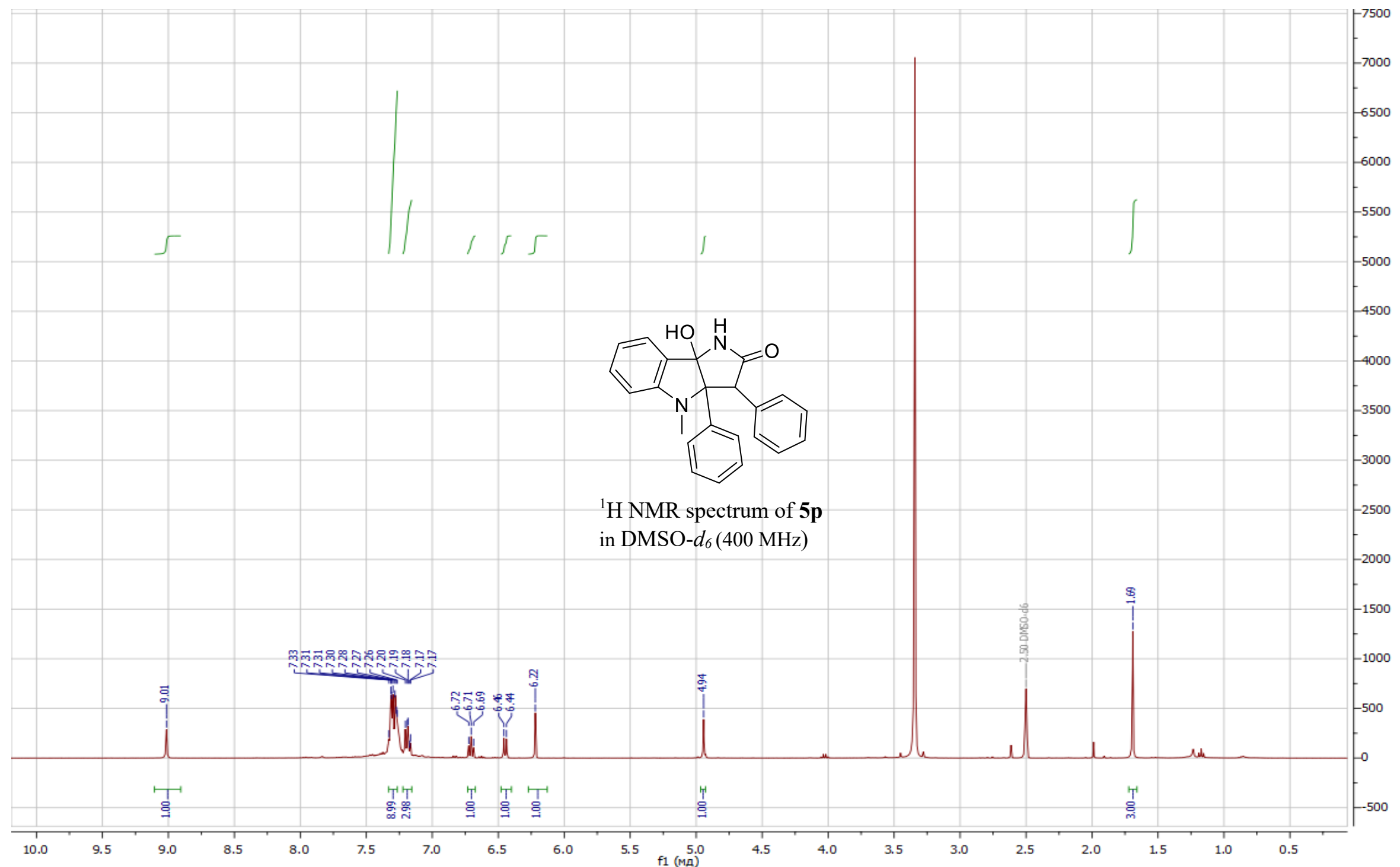




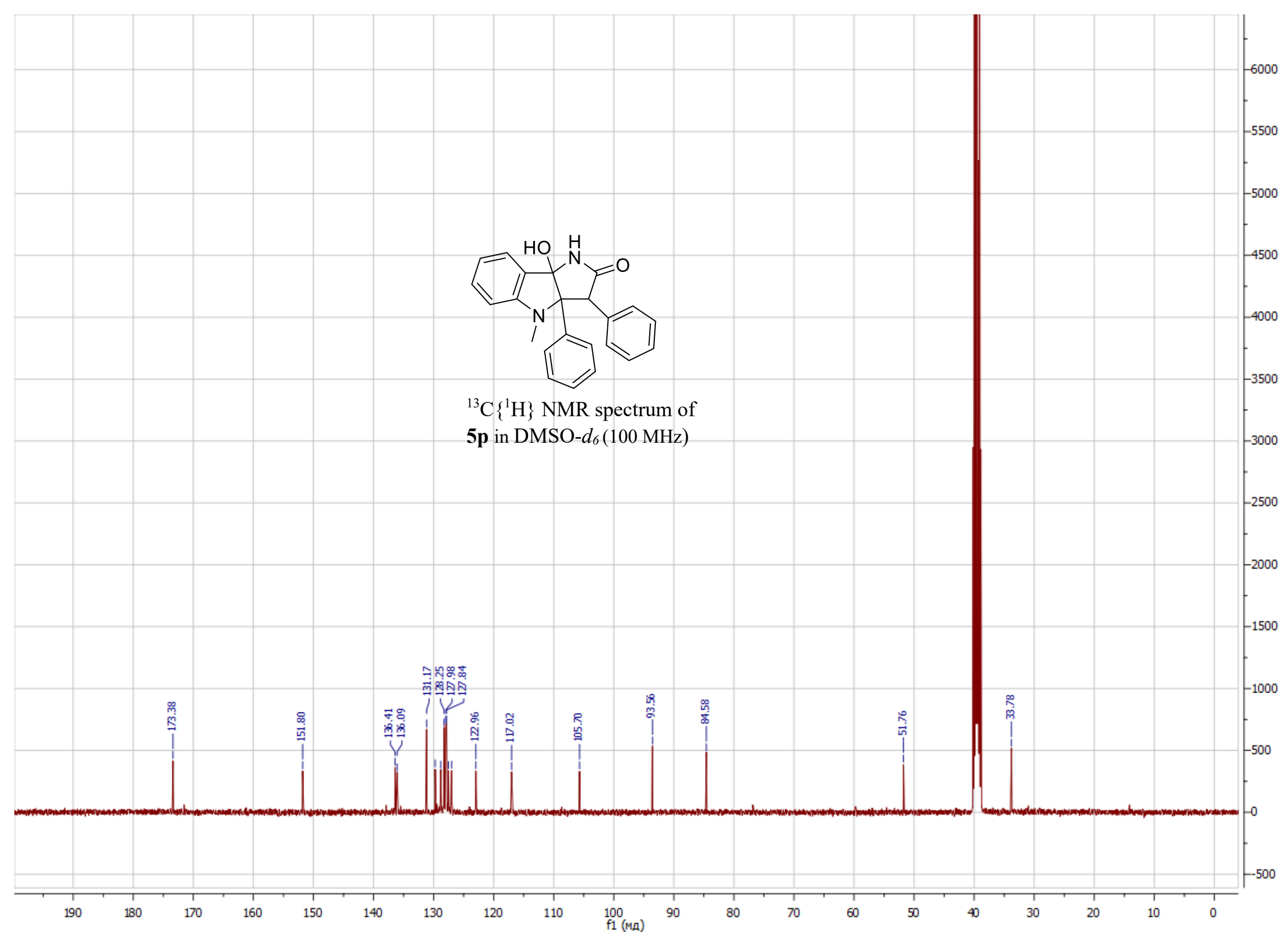




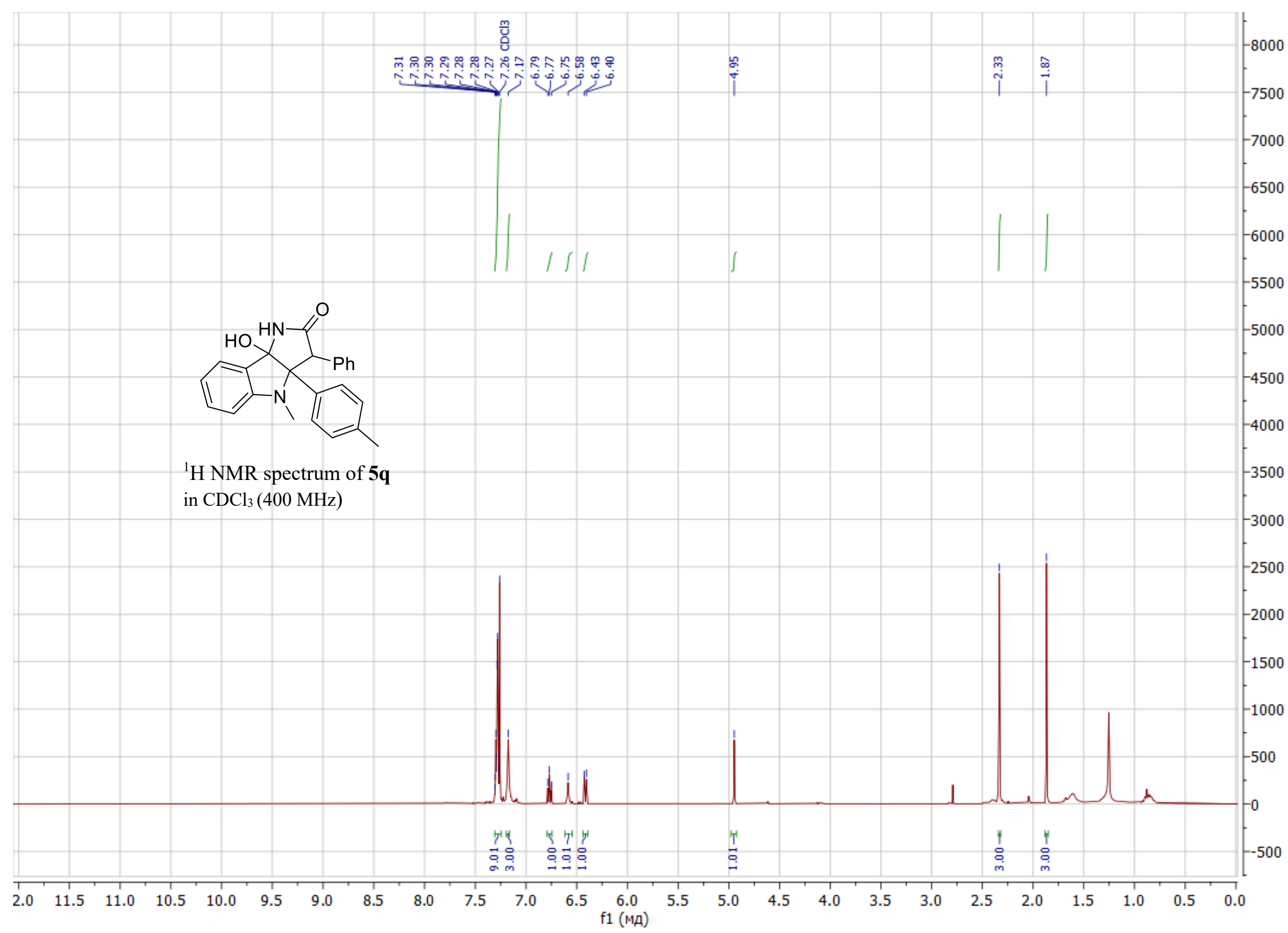




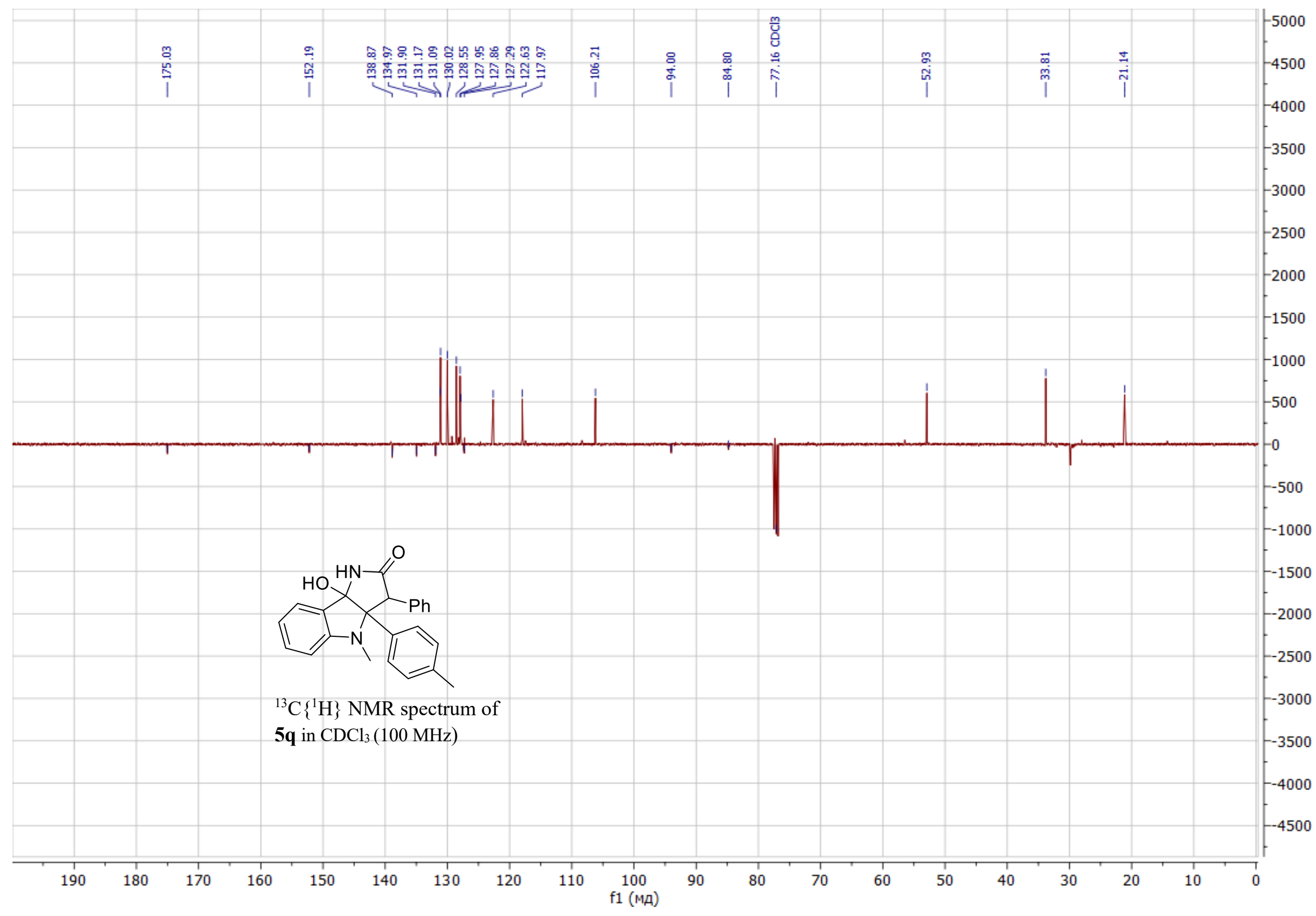




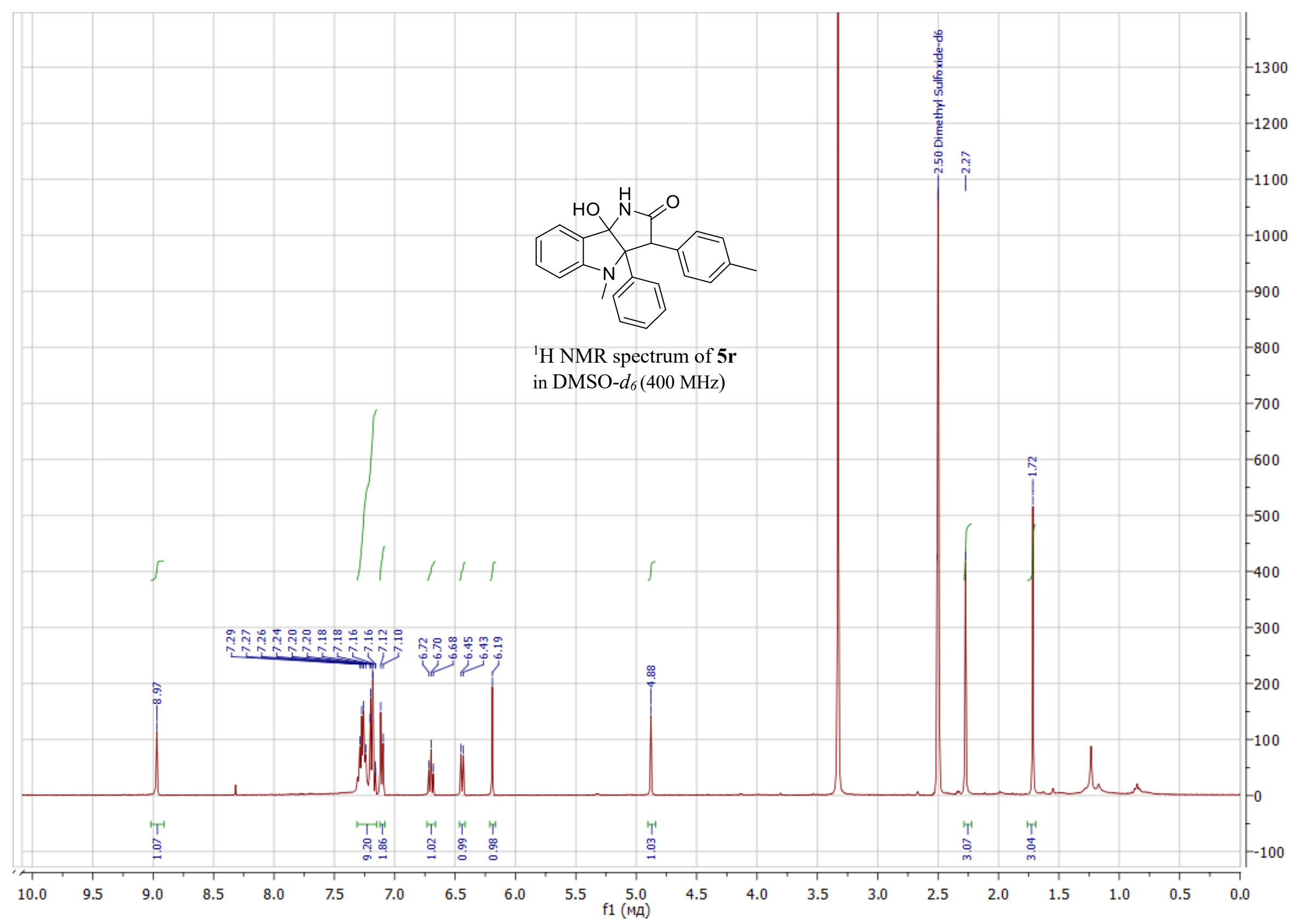




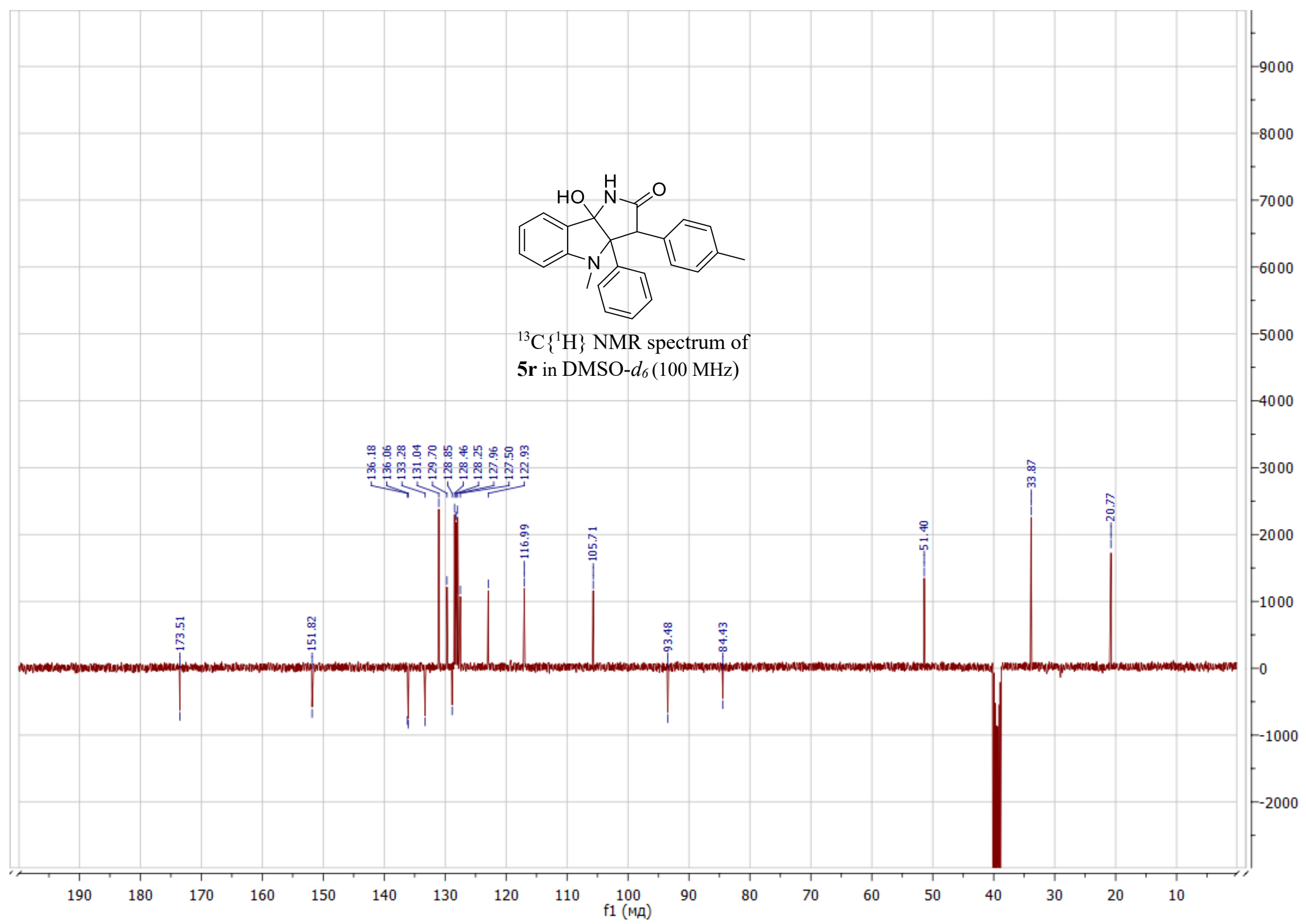




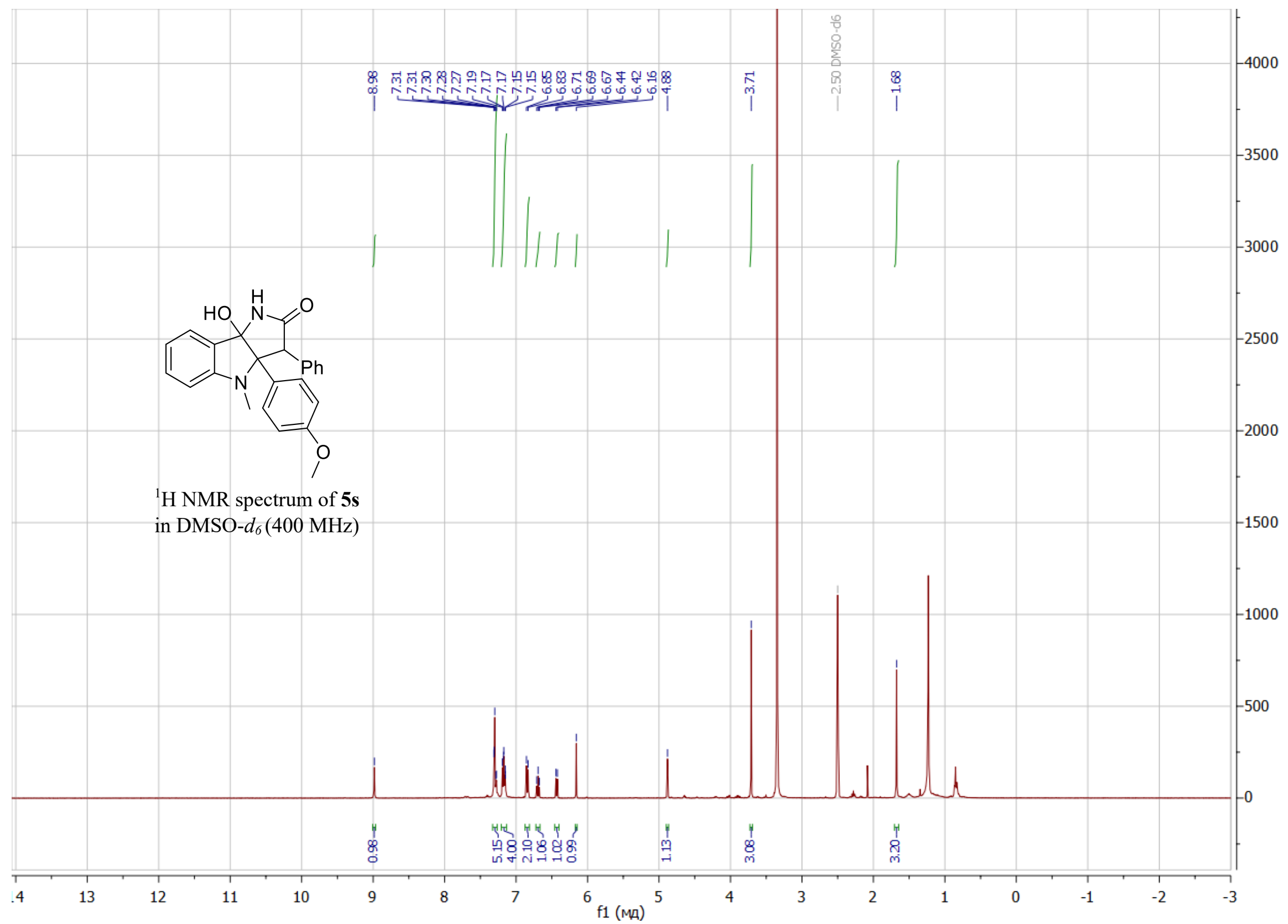




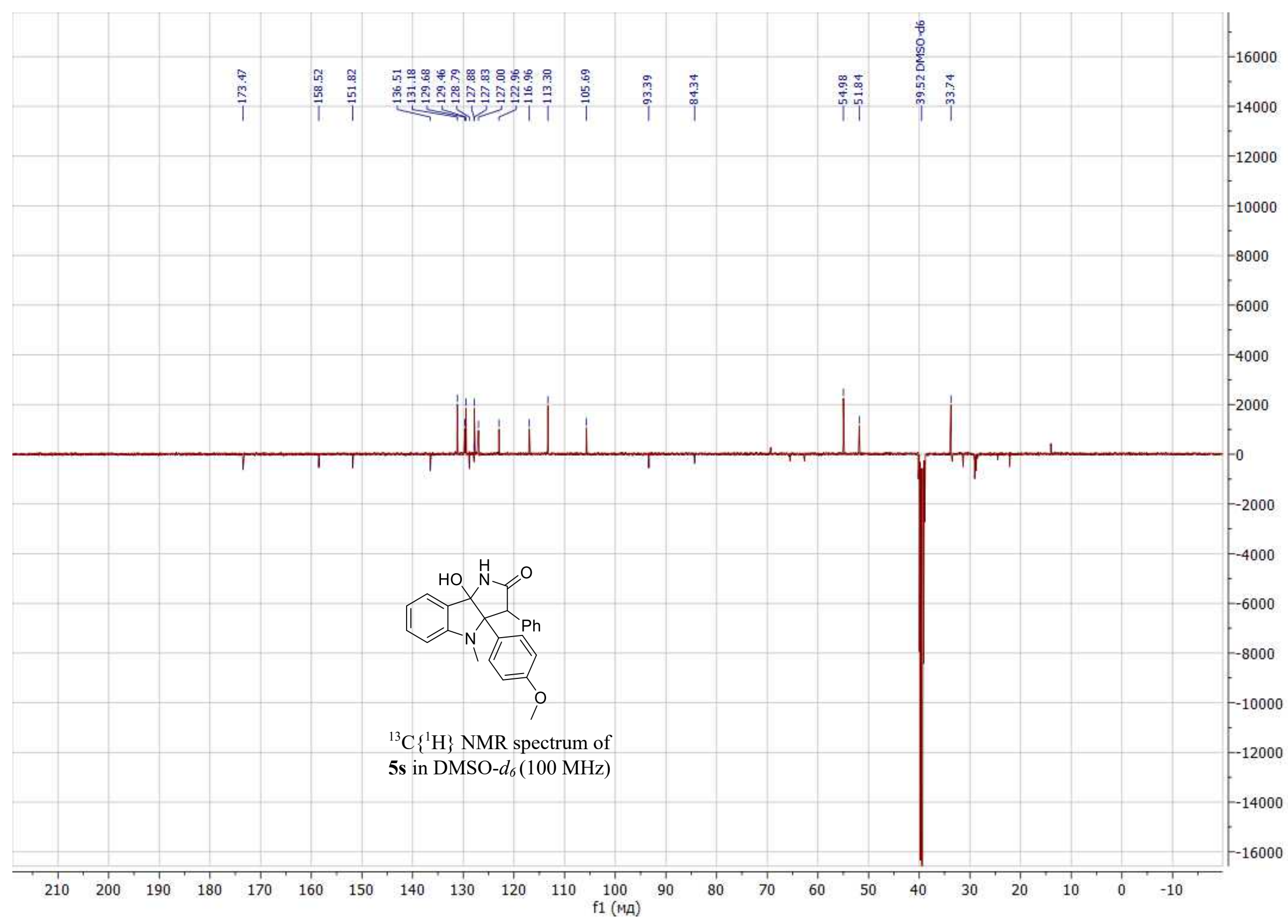




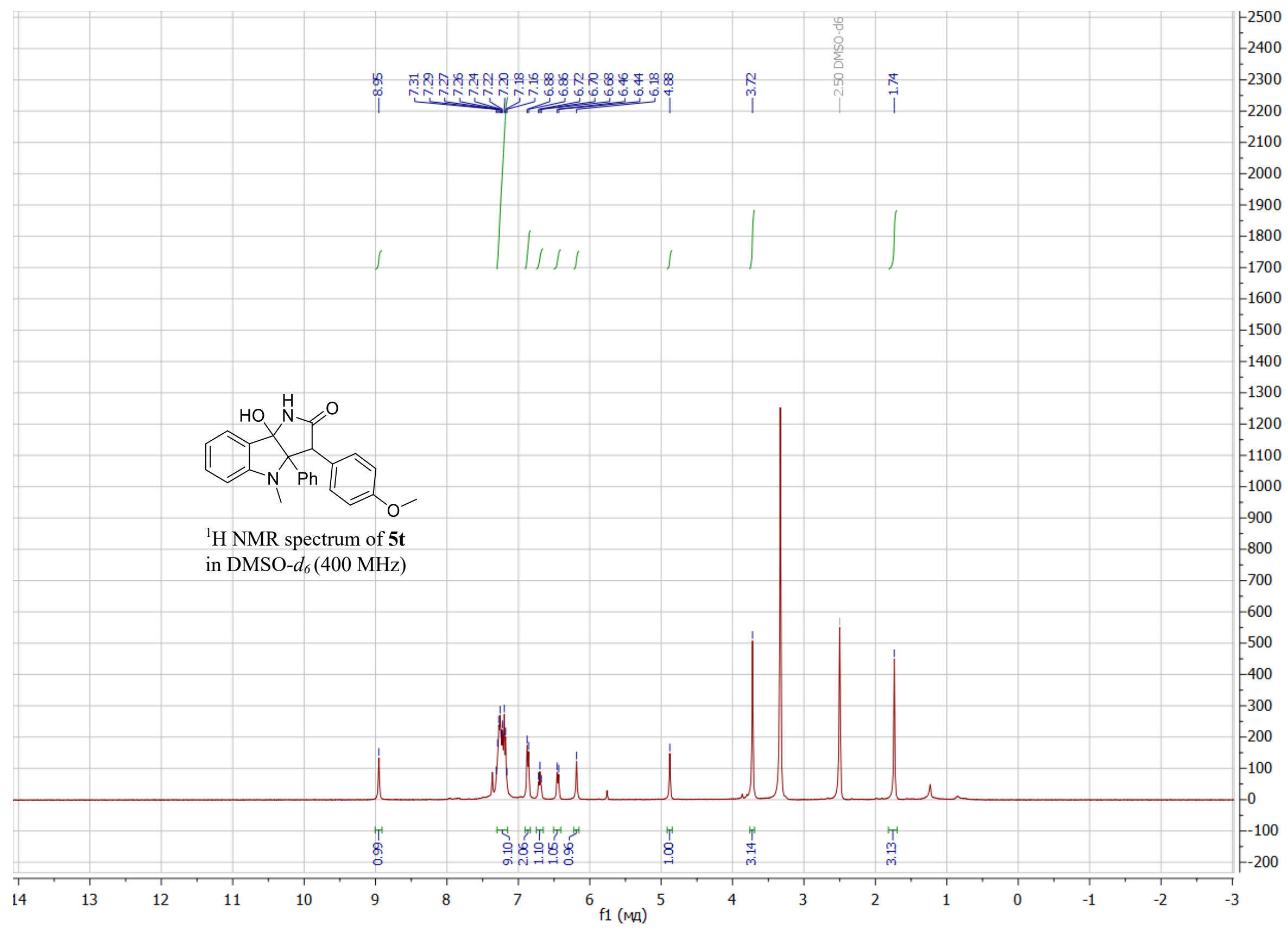




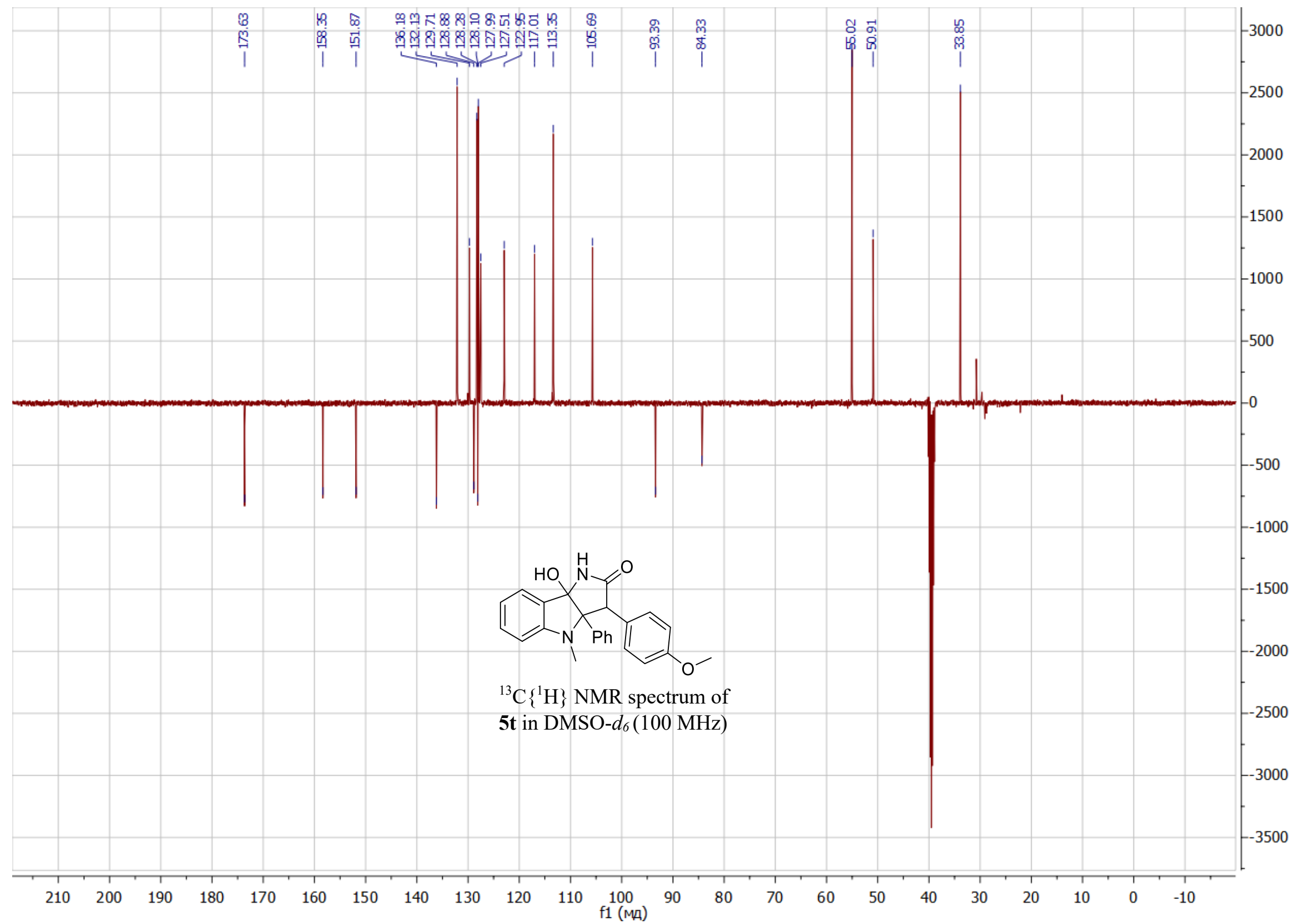




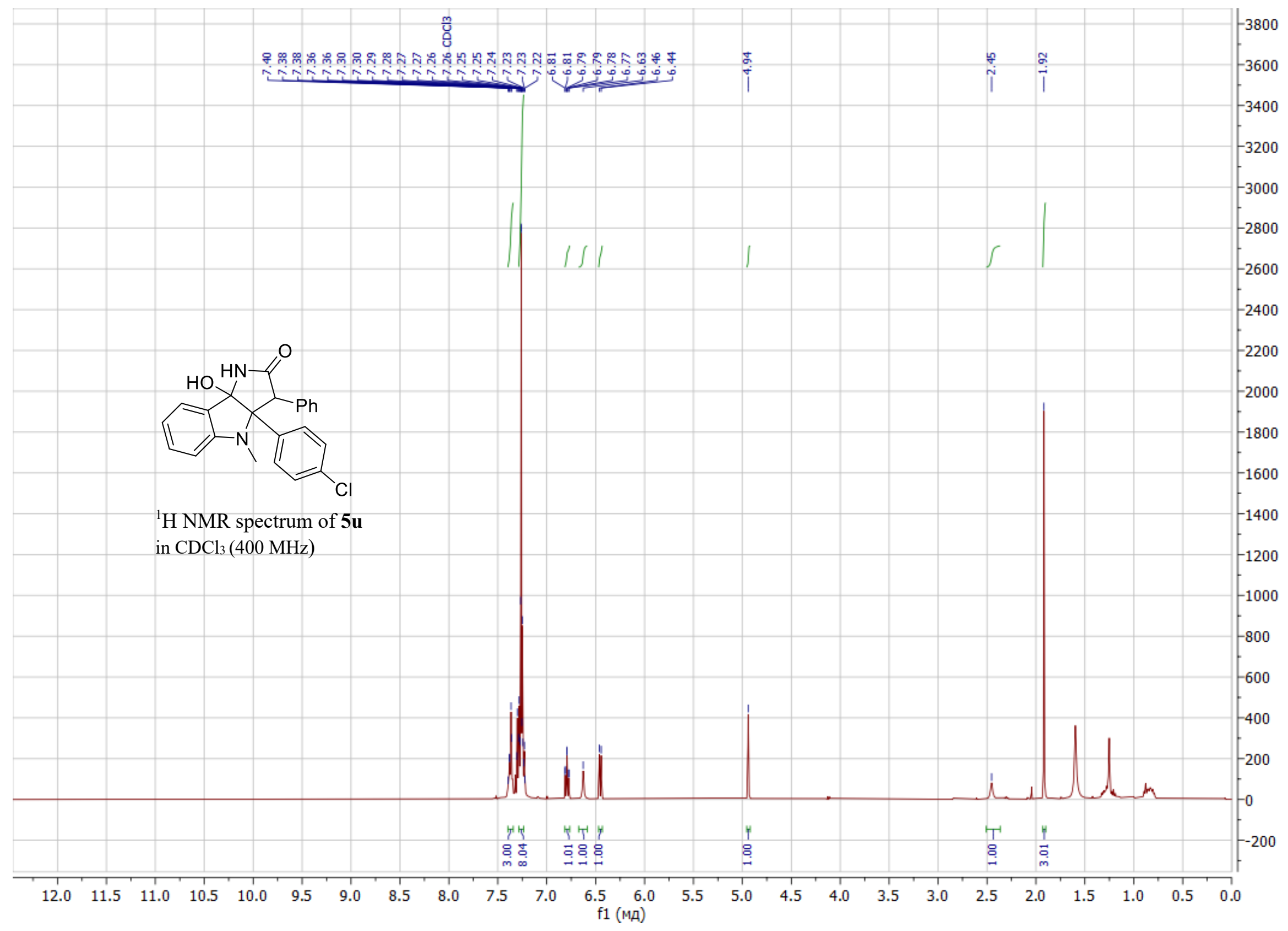




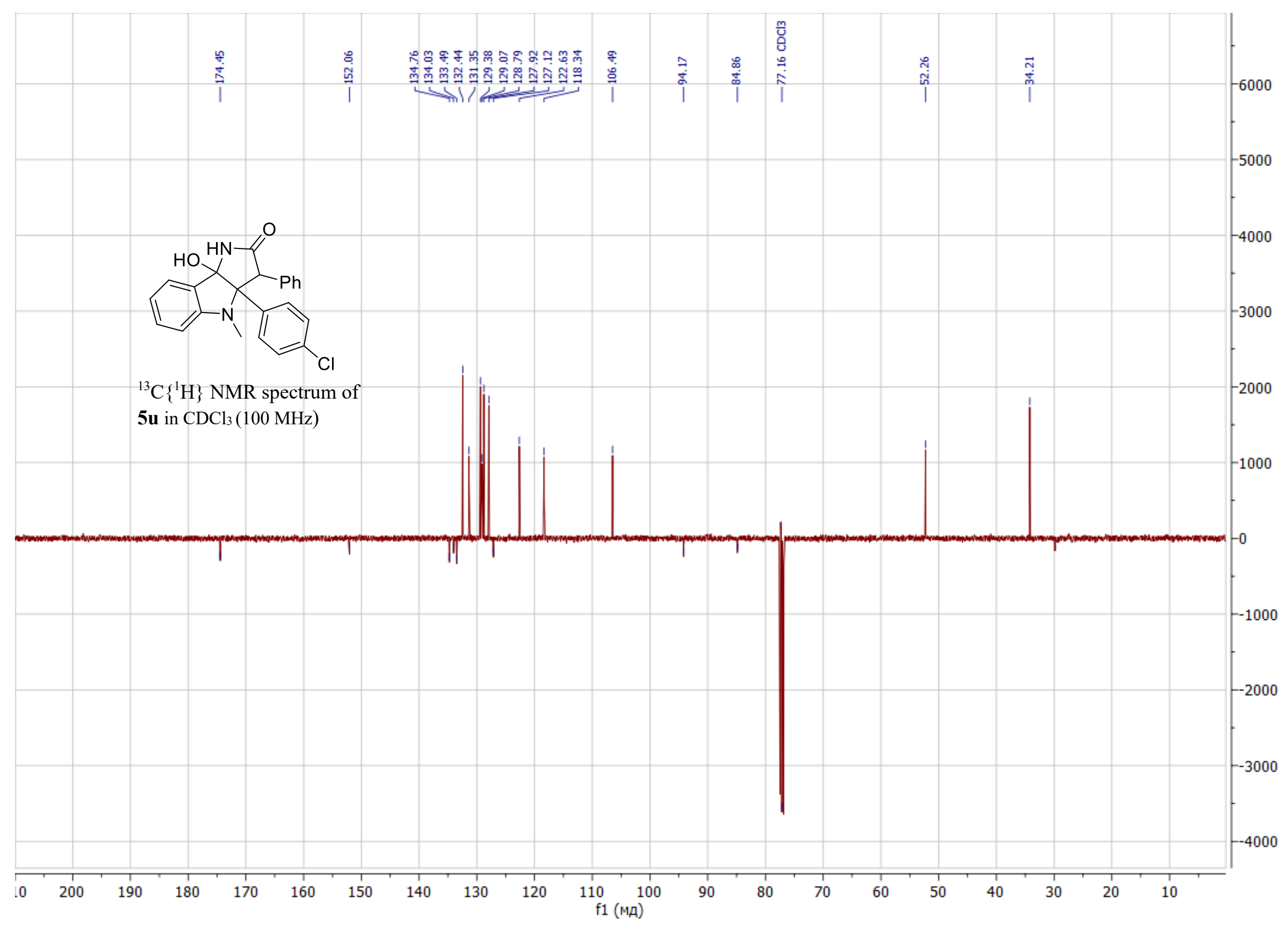




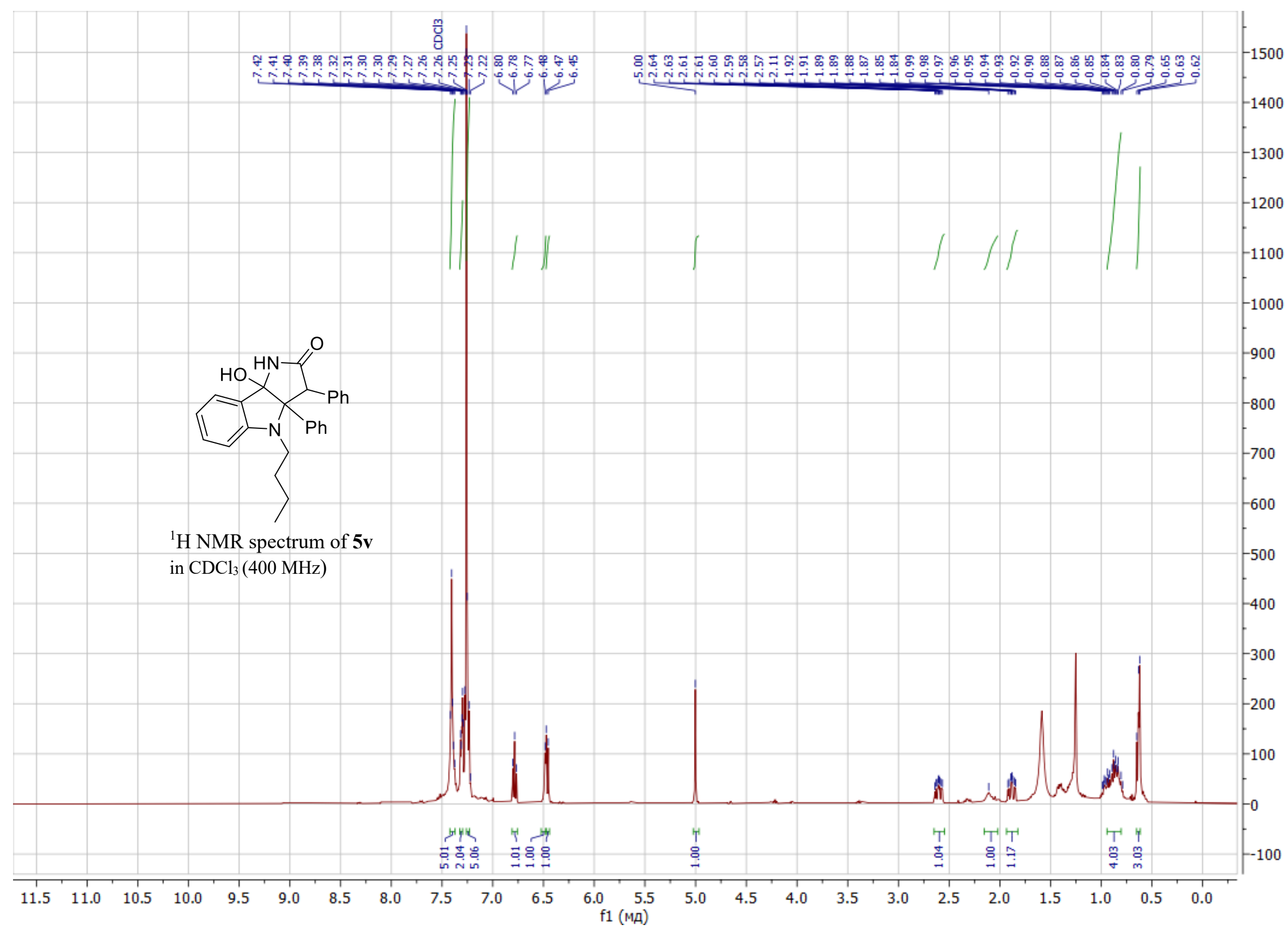




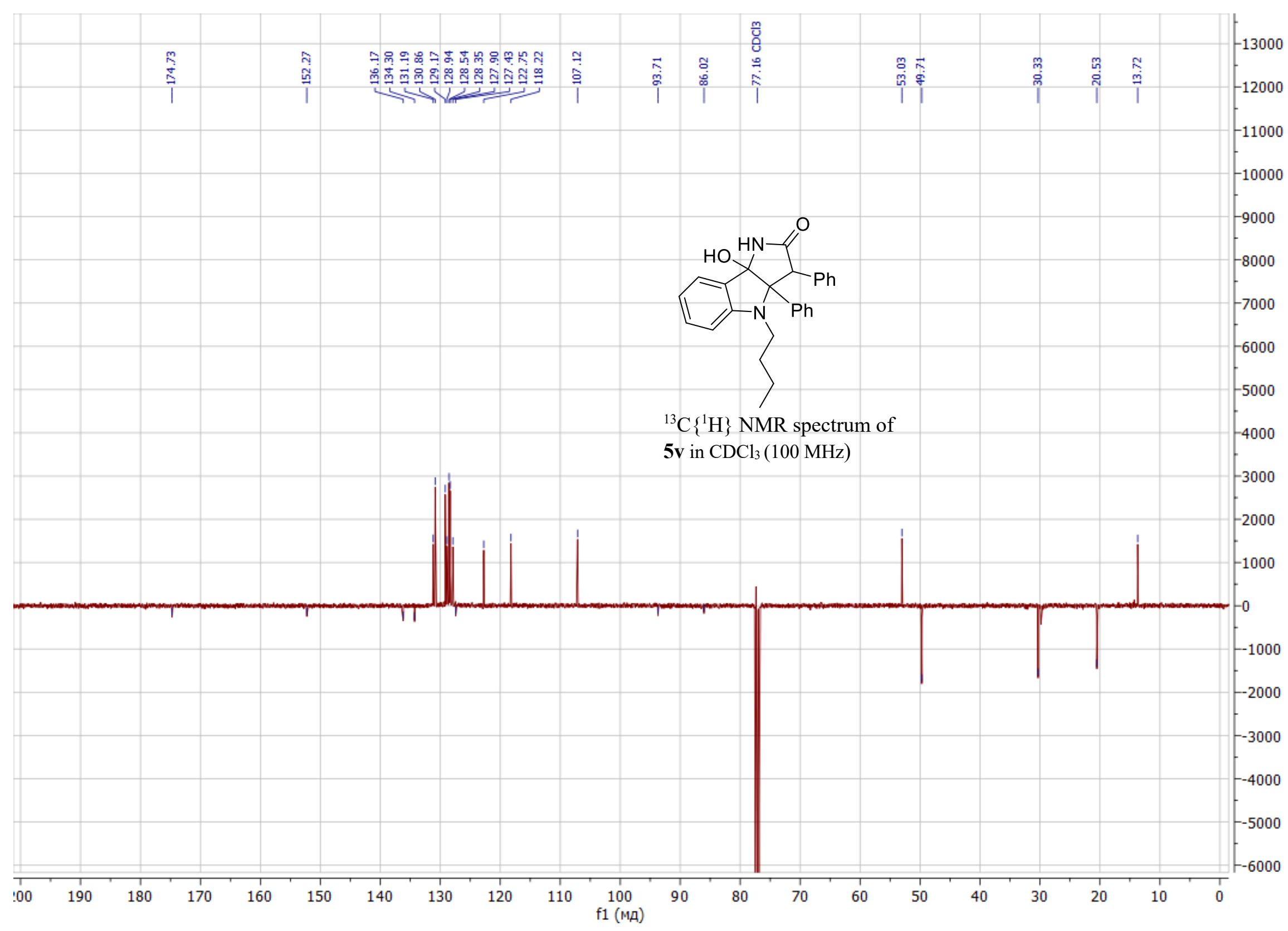




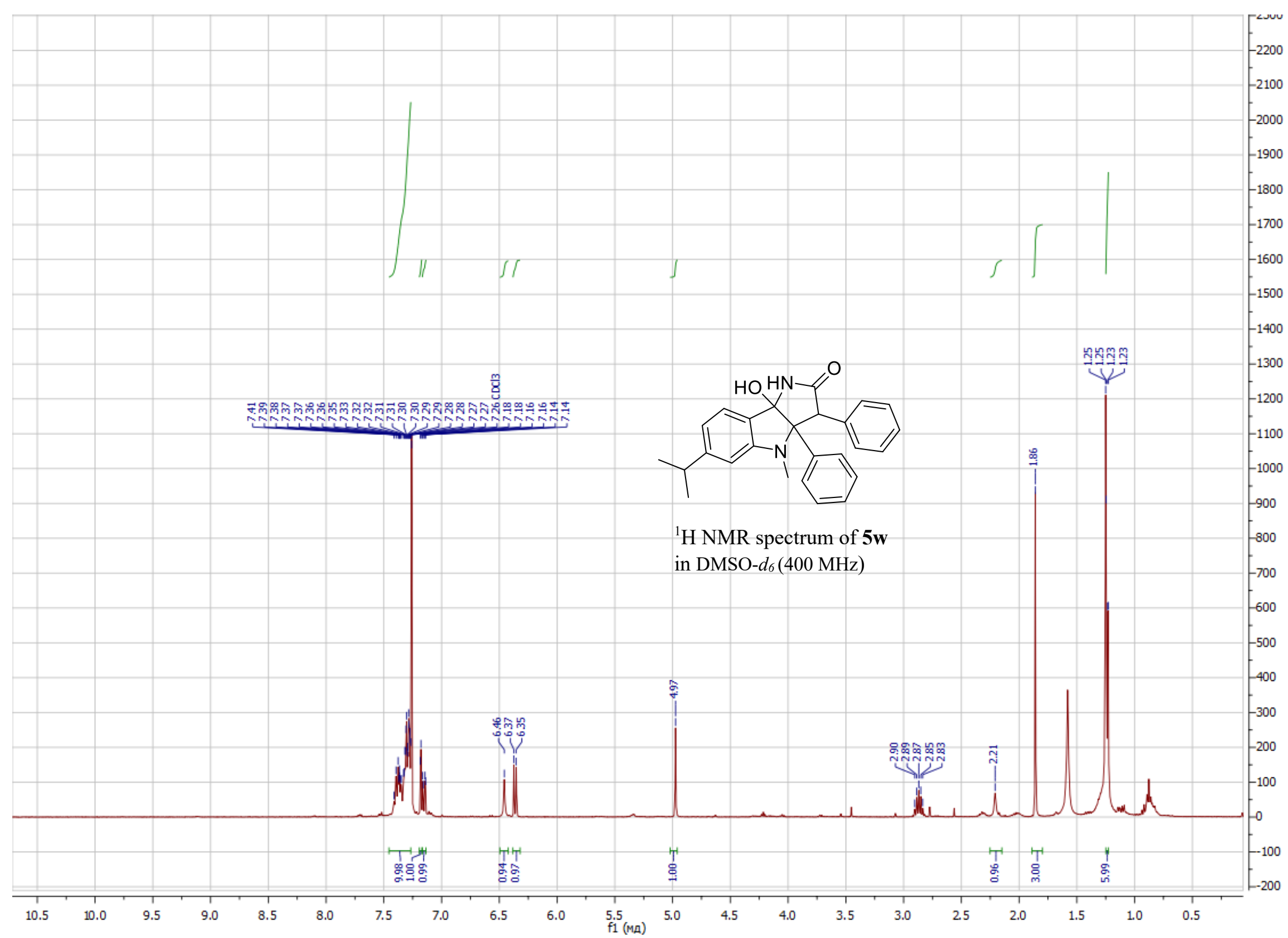



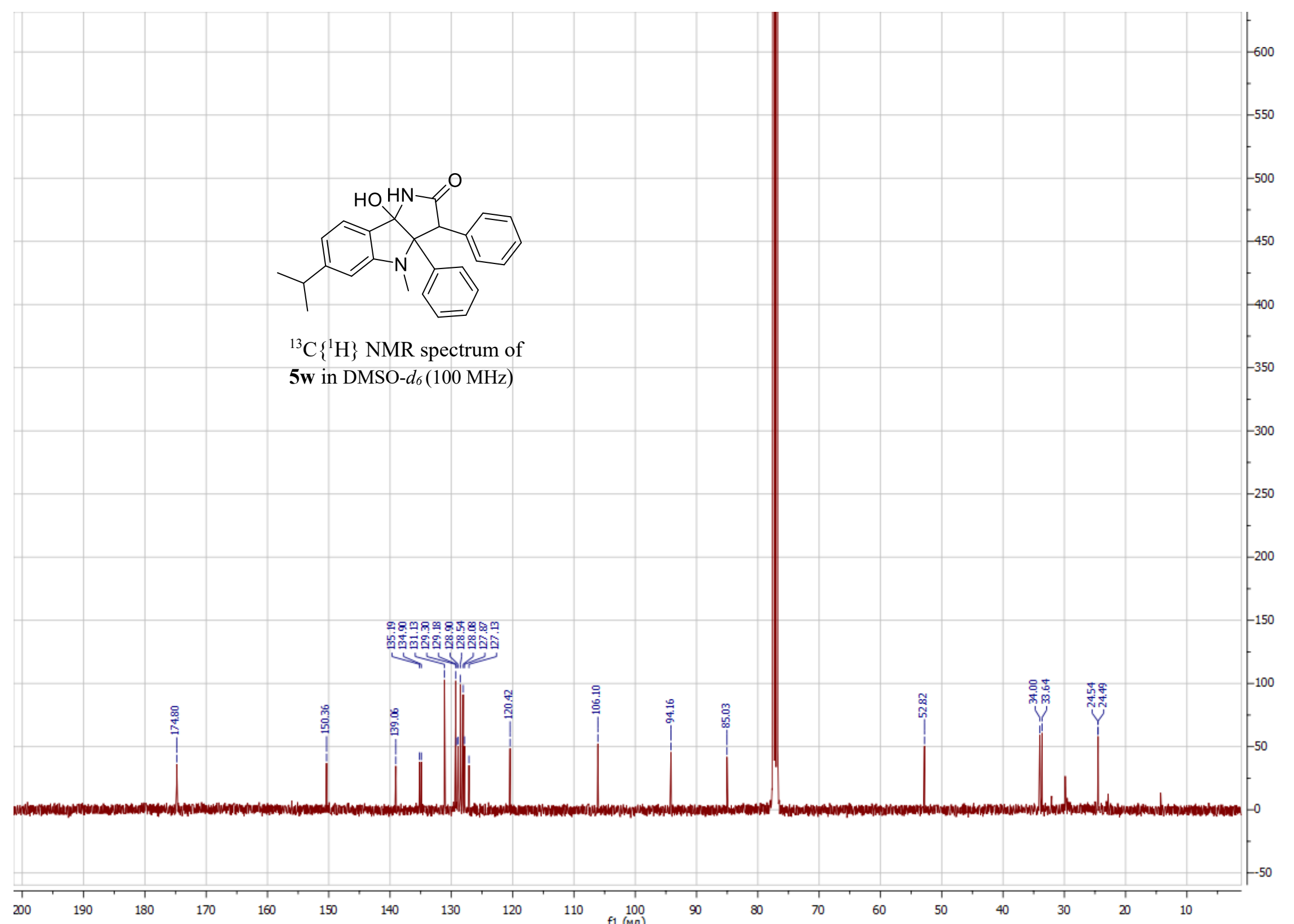


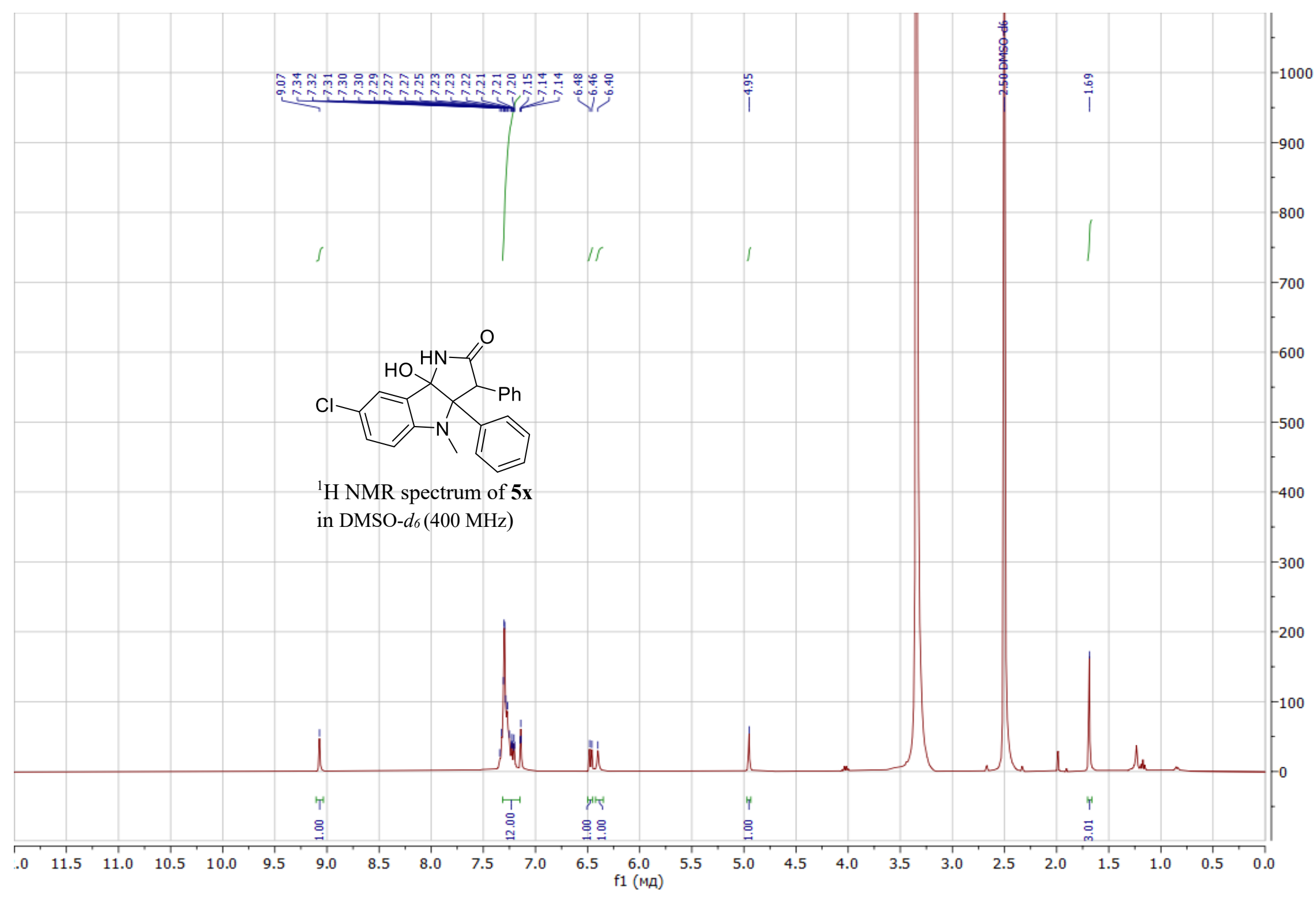




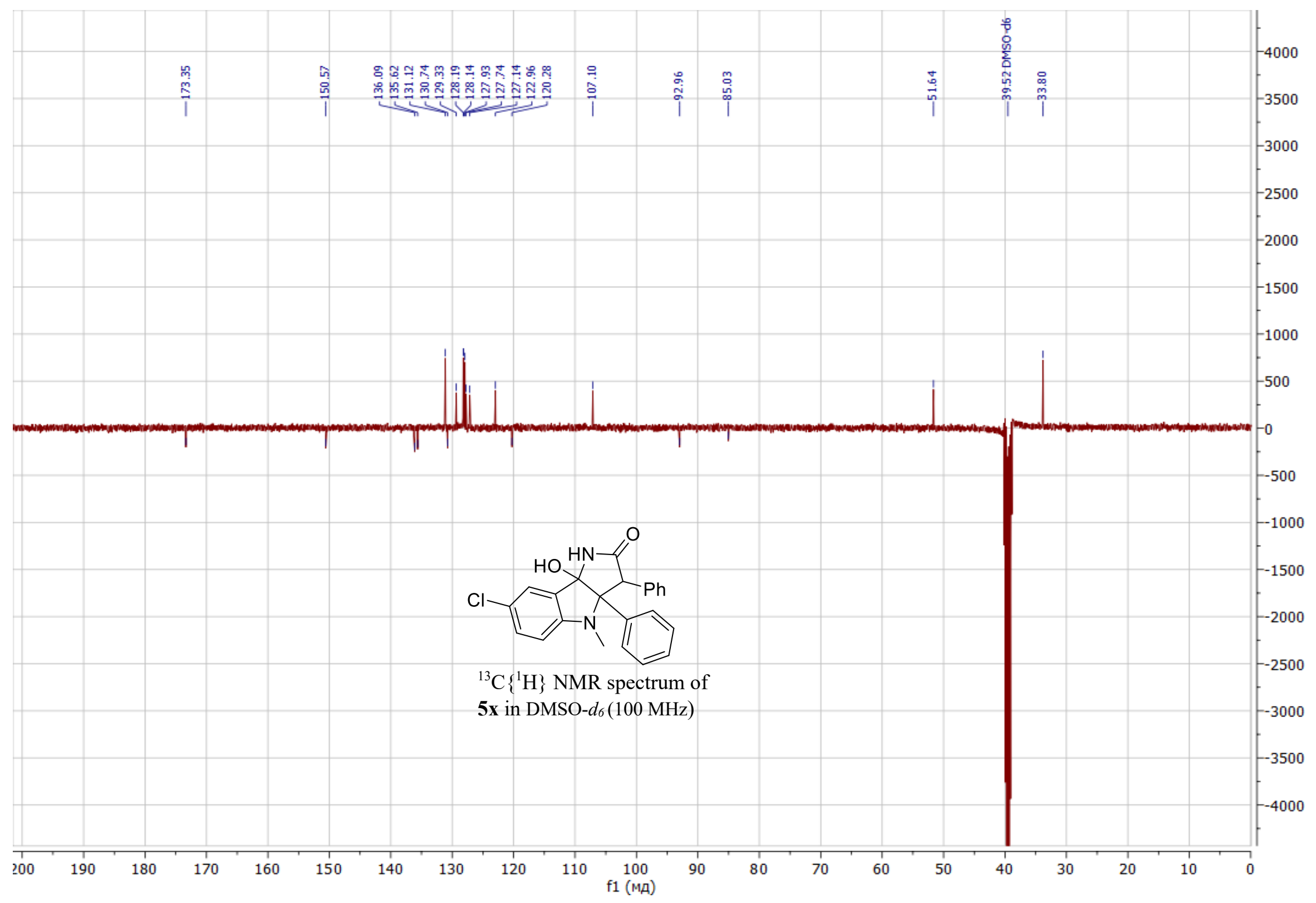


${ }^{1} \mathrm{H}$ and ${ }^{13} \mathrm{C}$ Spectral Charts for 3-hydroxyindolin-2-ones 6

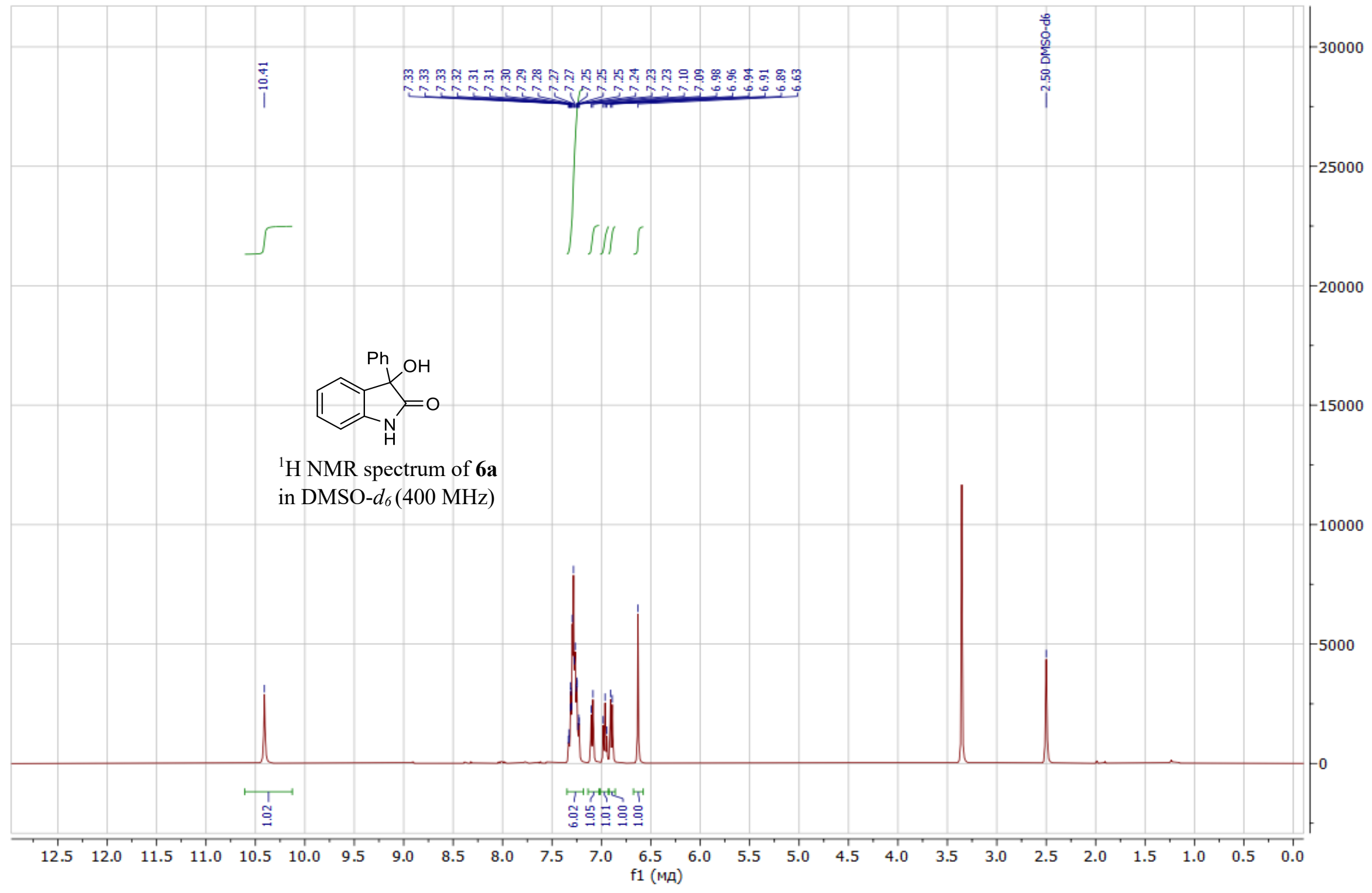




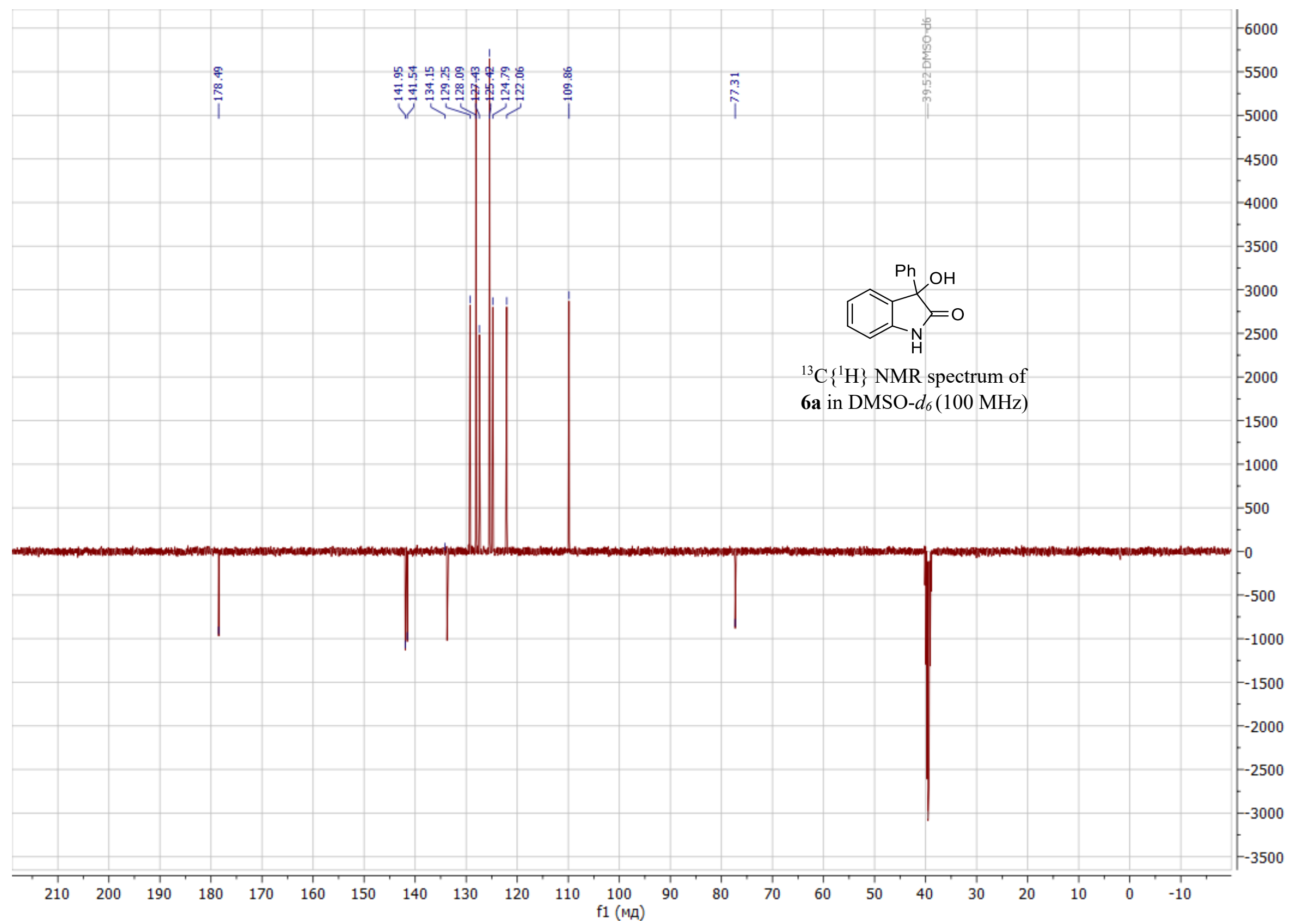




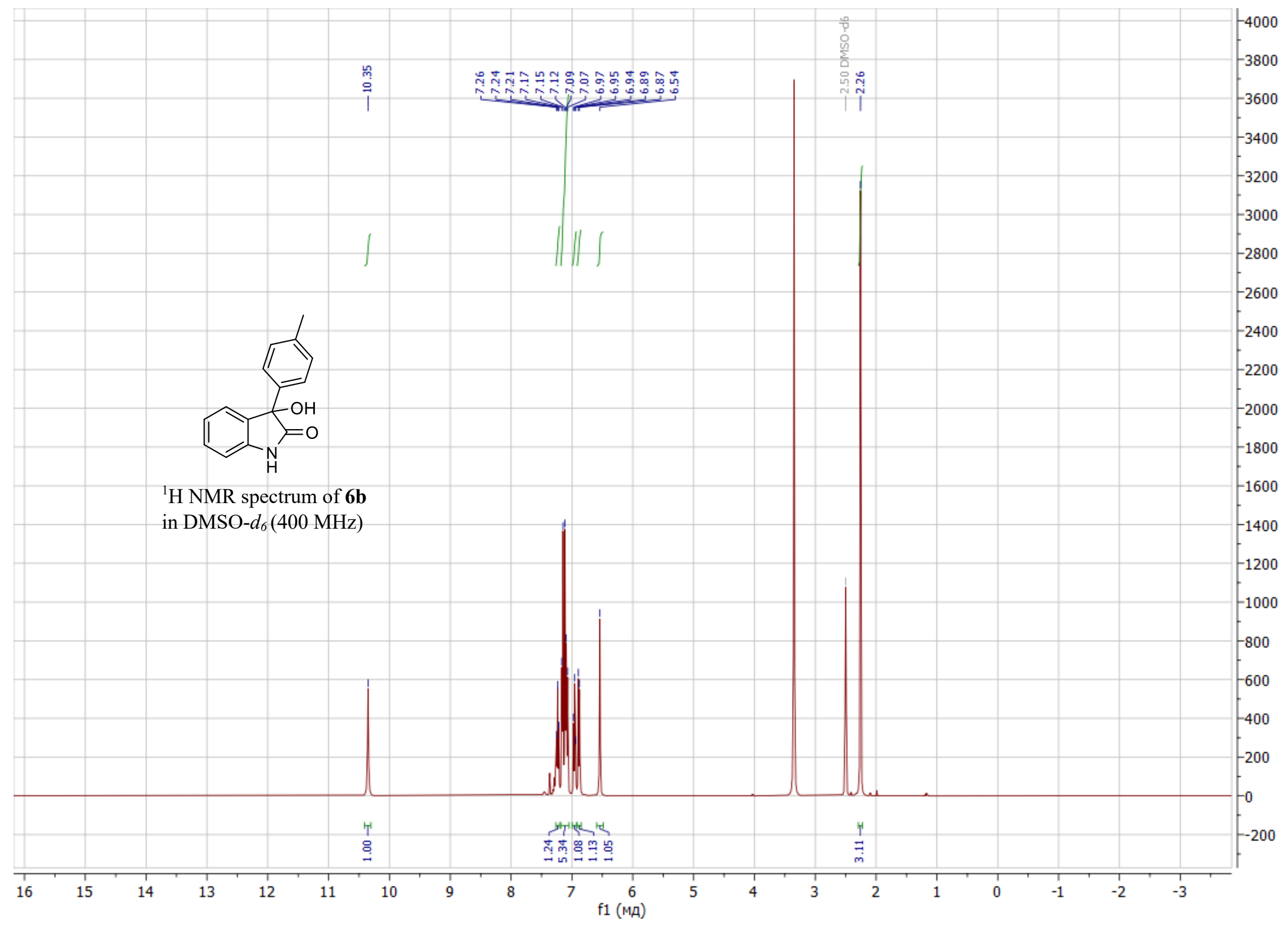




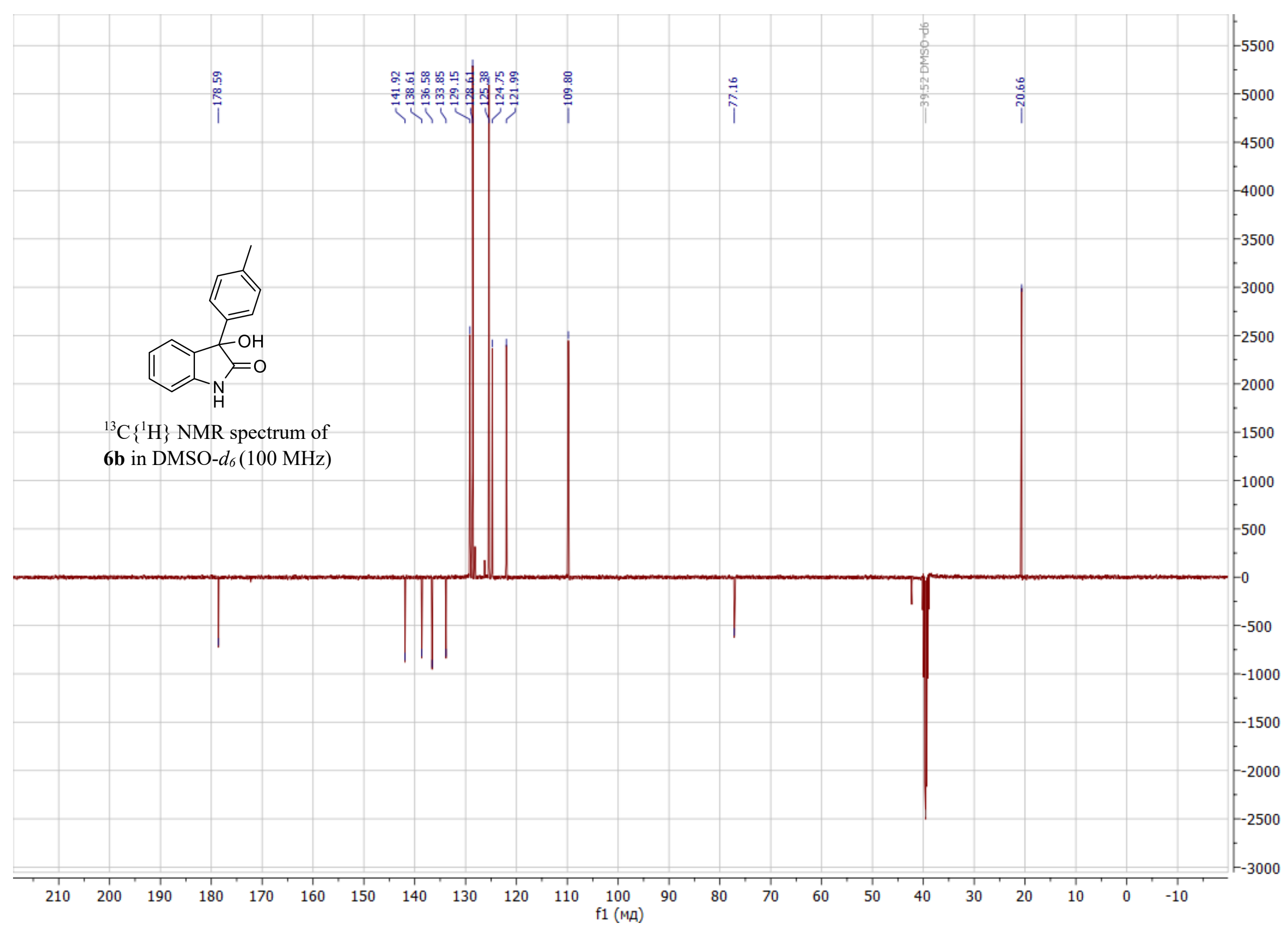




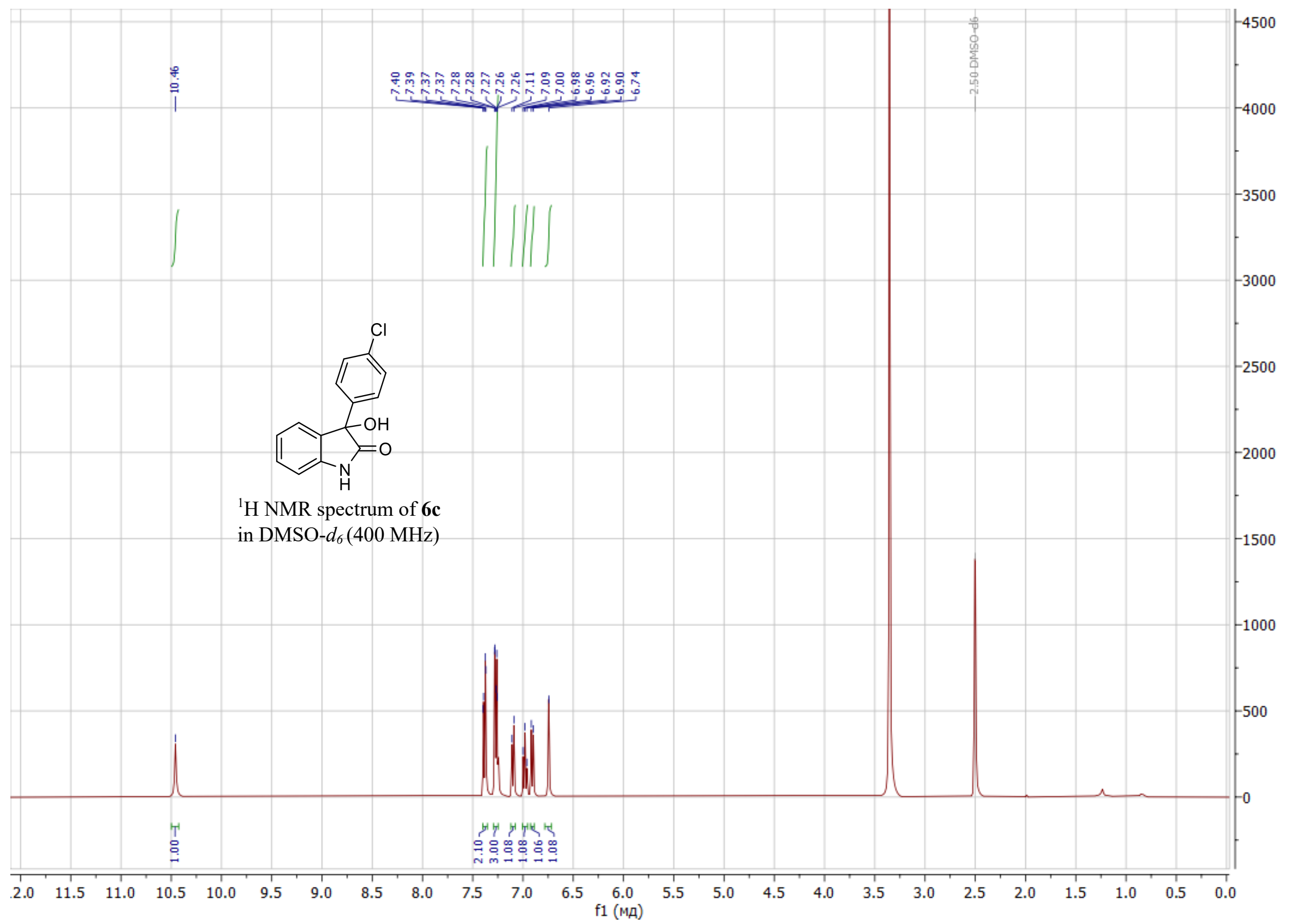




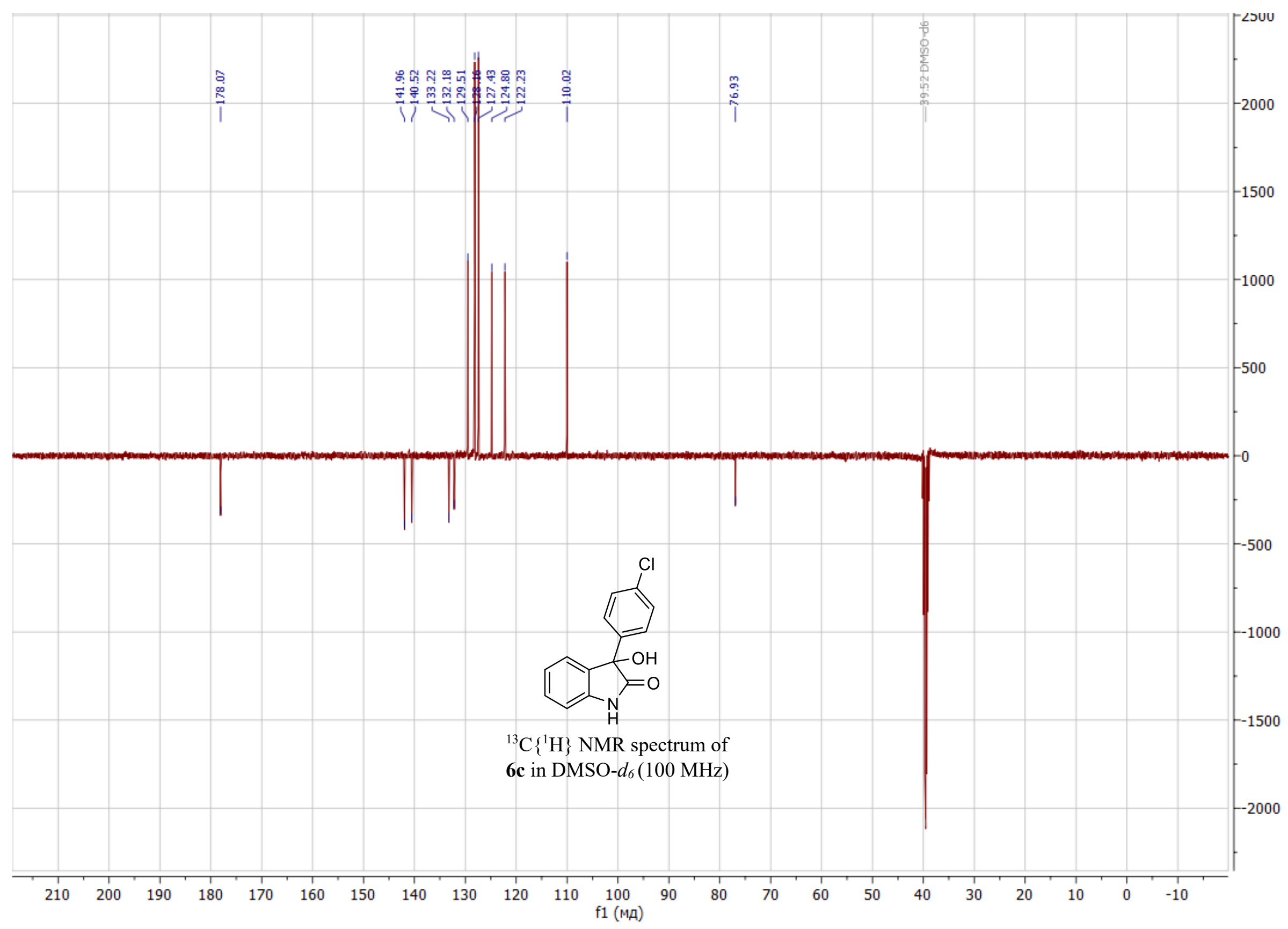




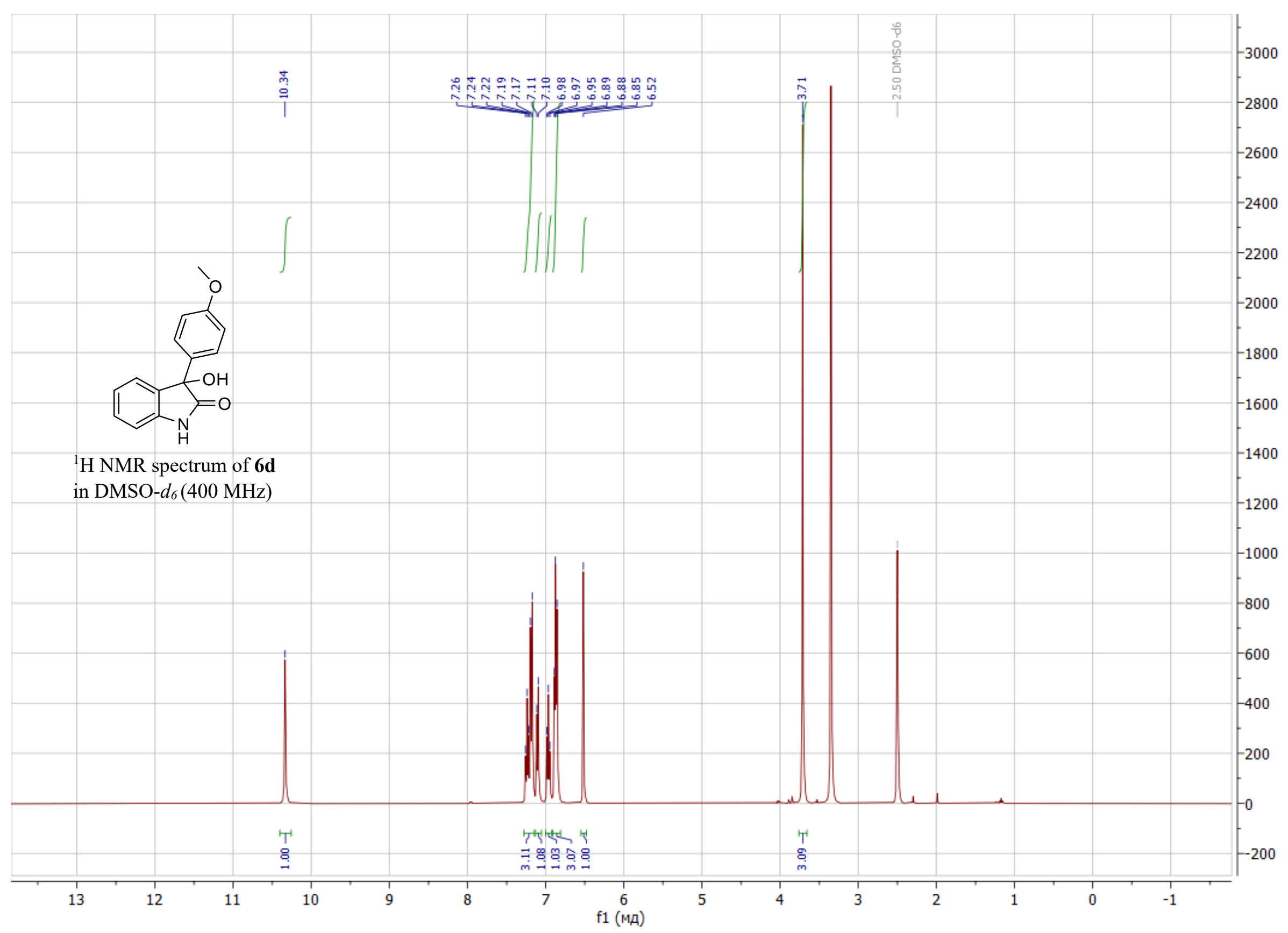




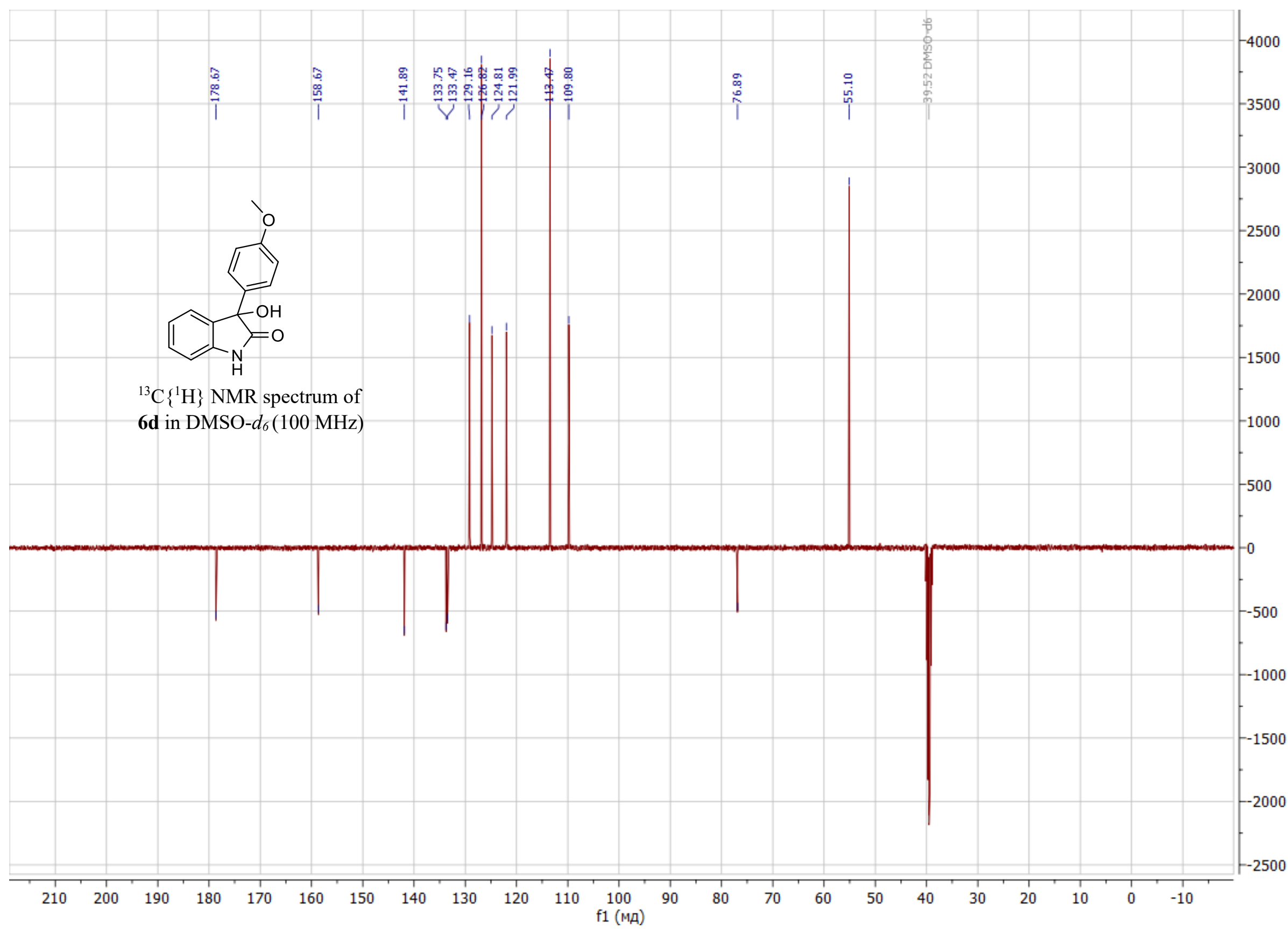




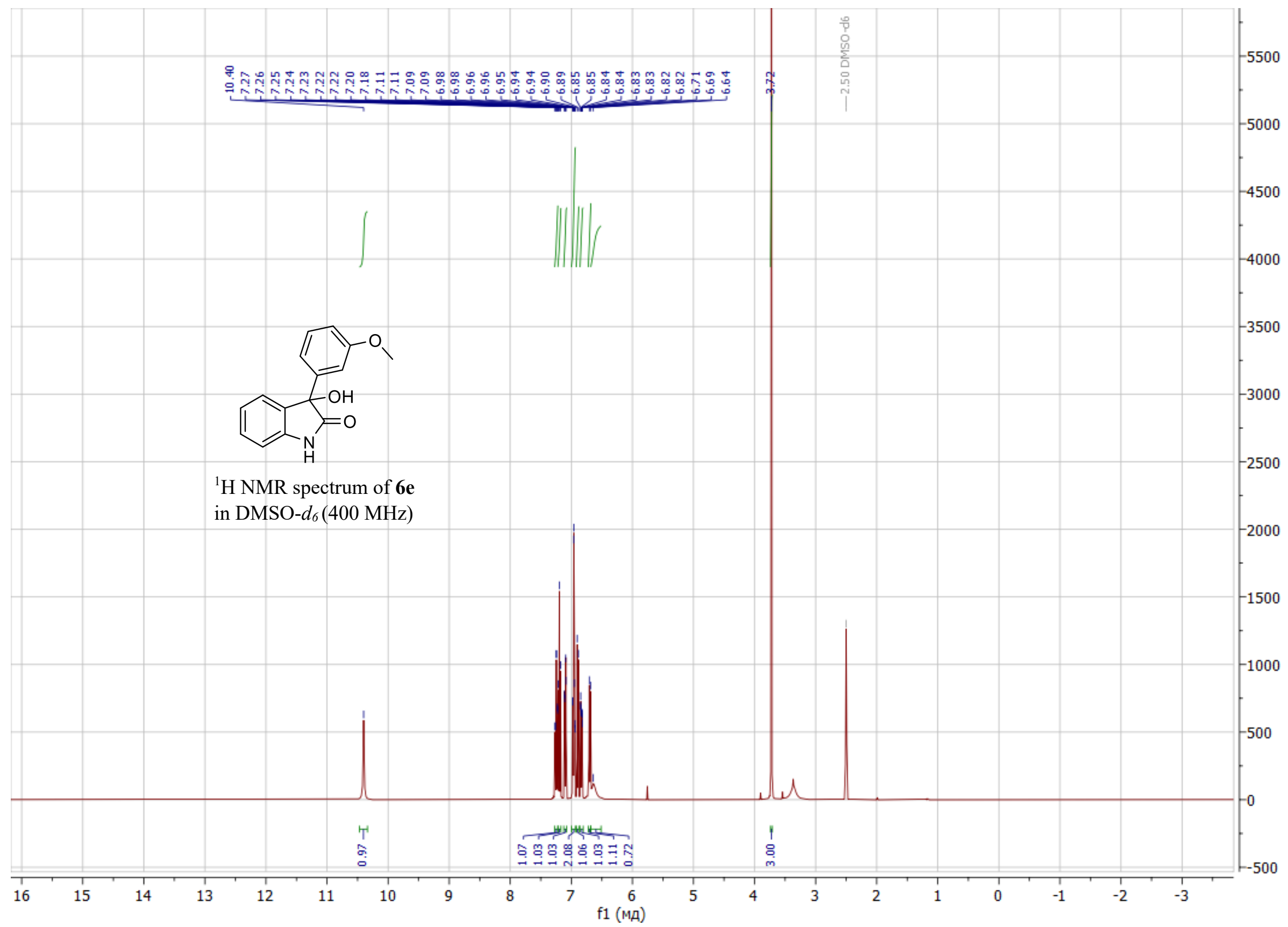




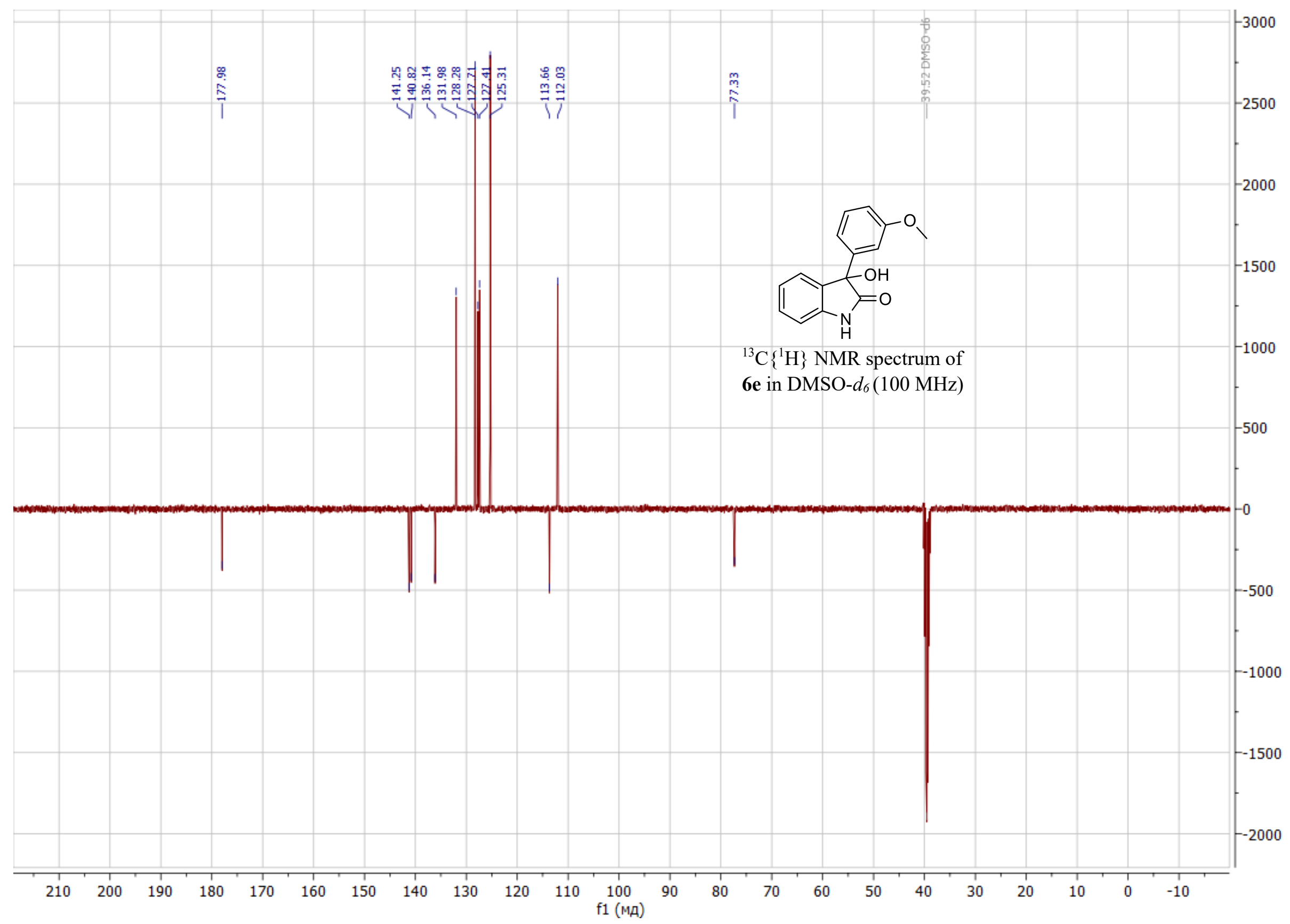




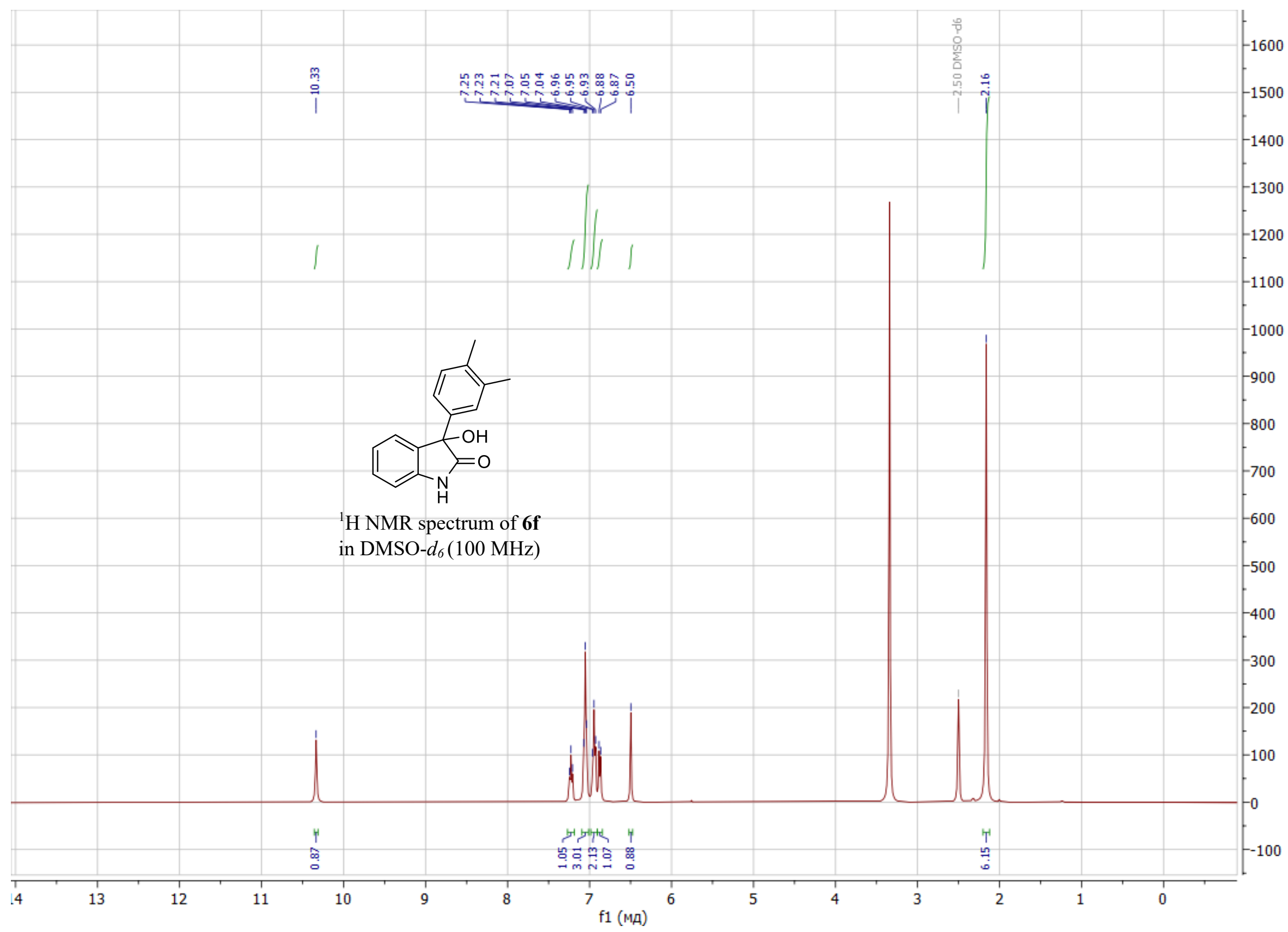




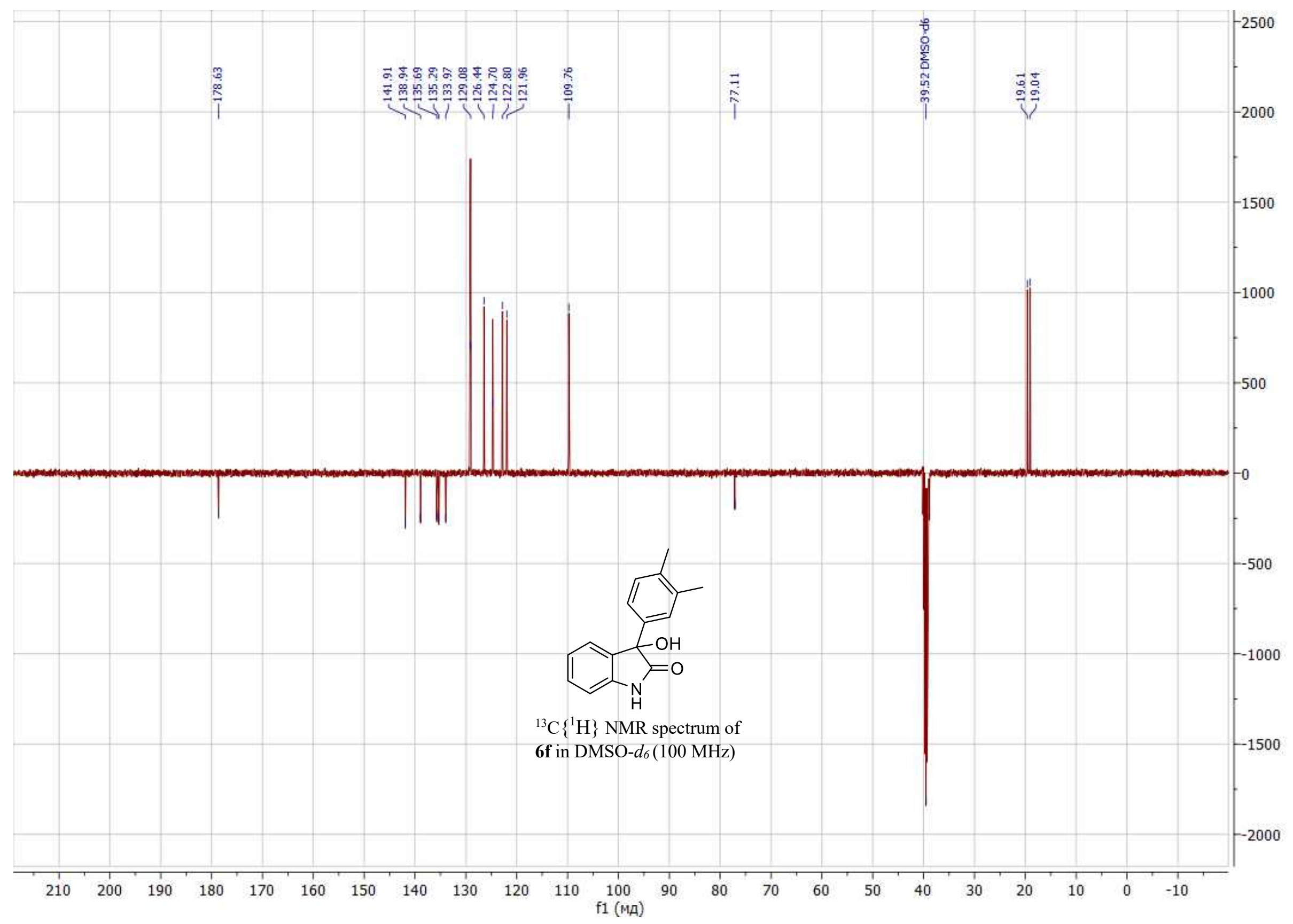




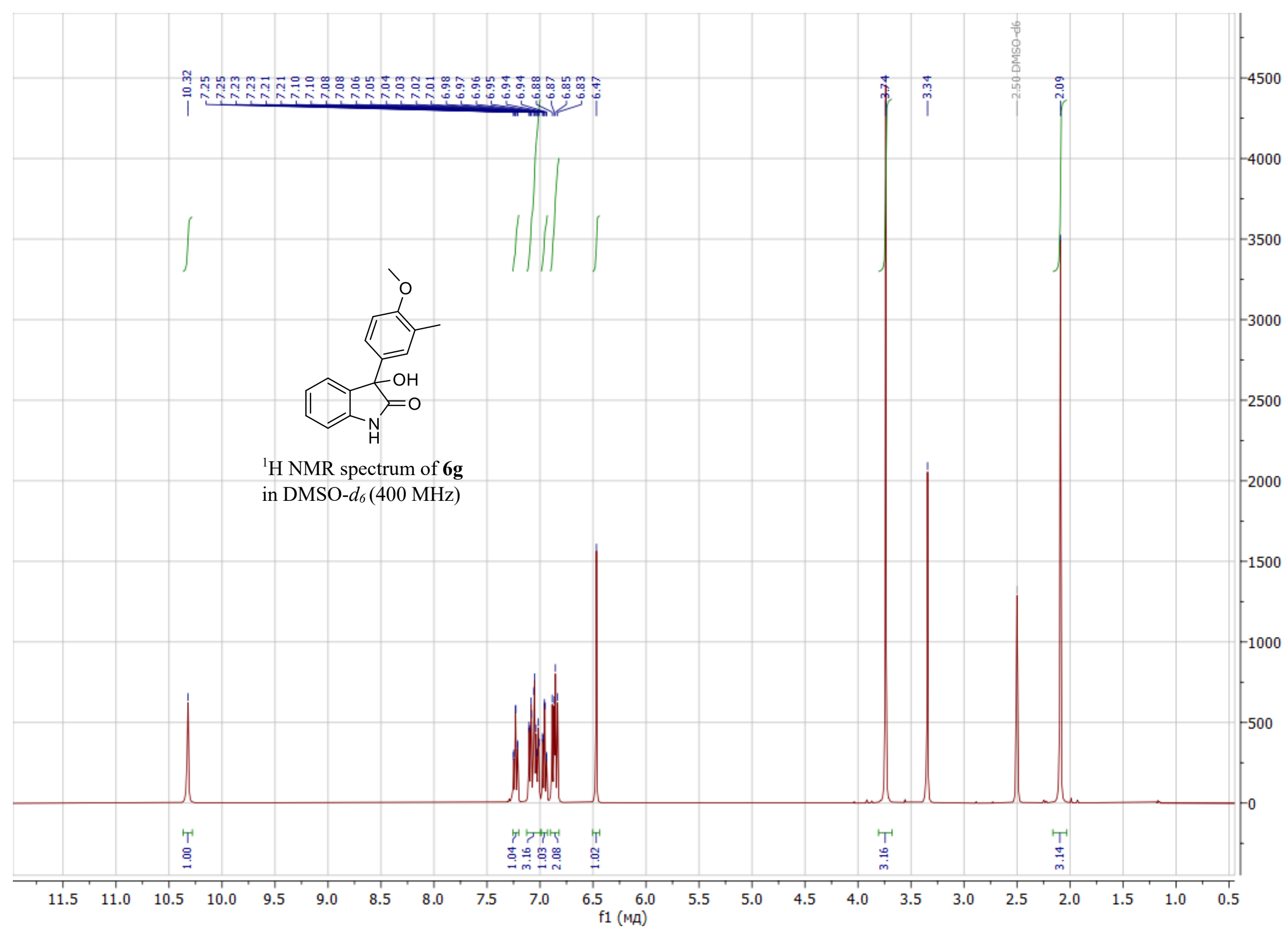




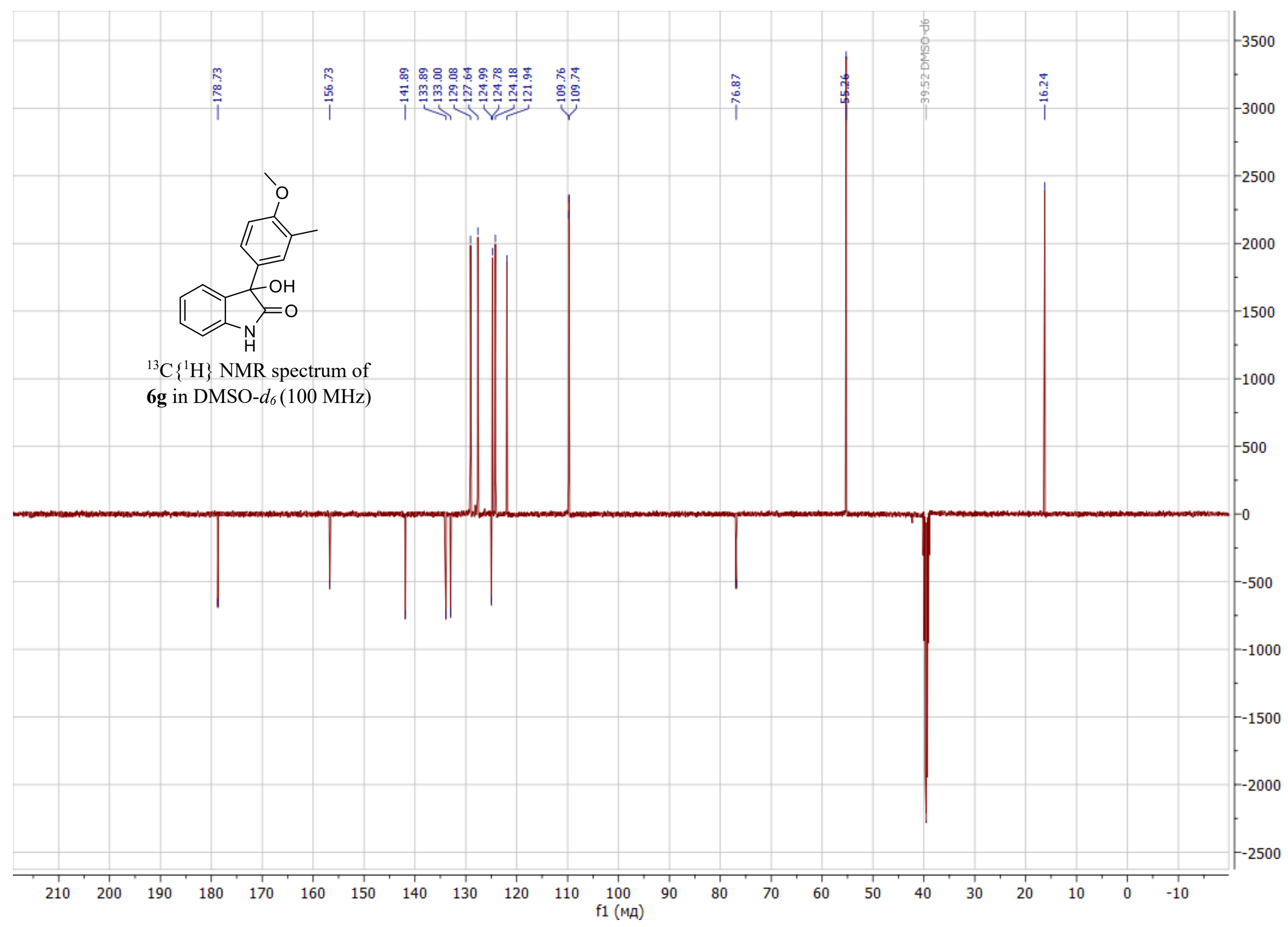




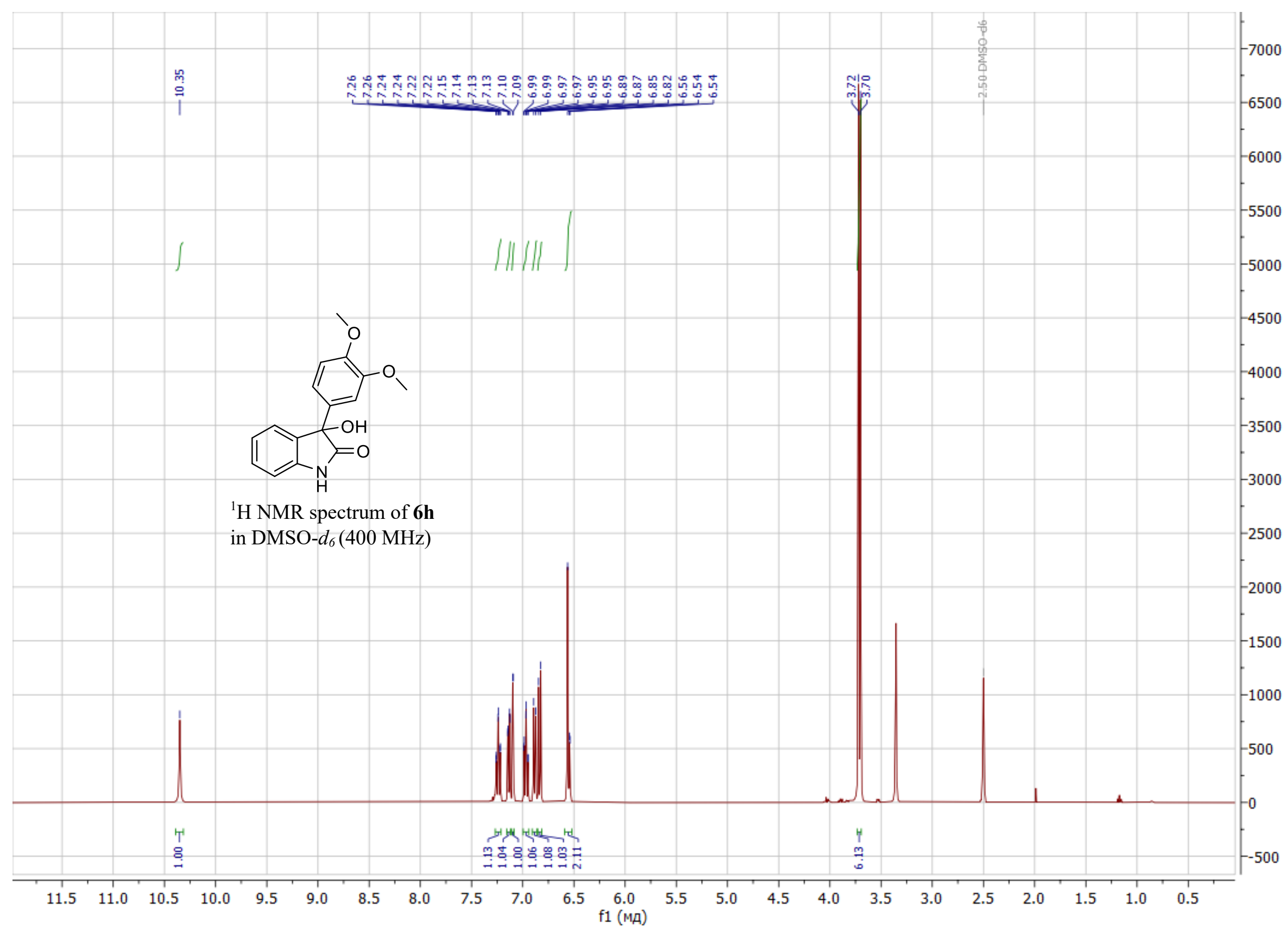




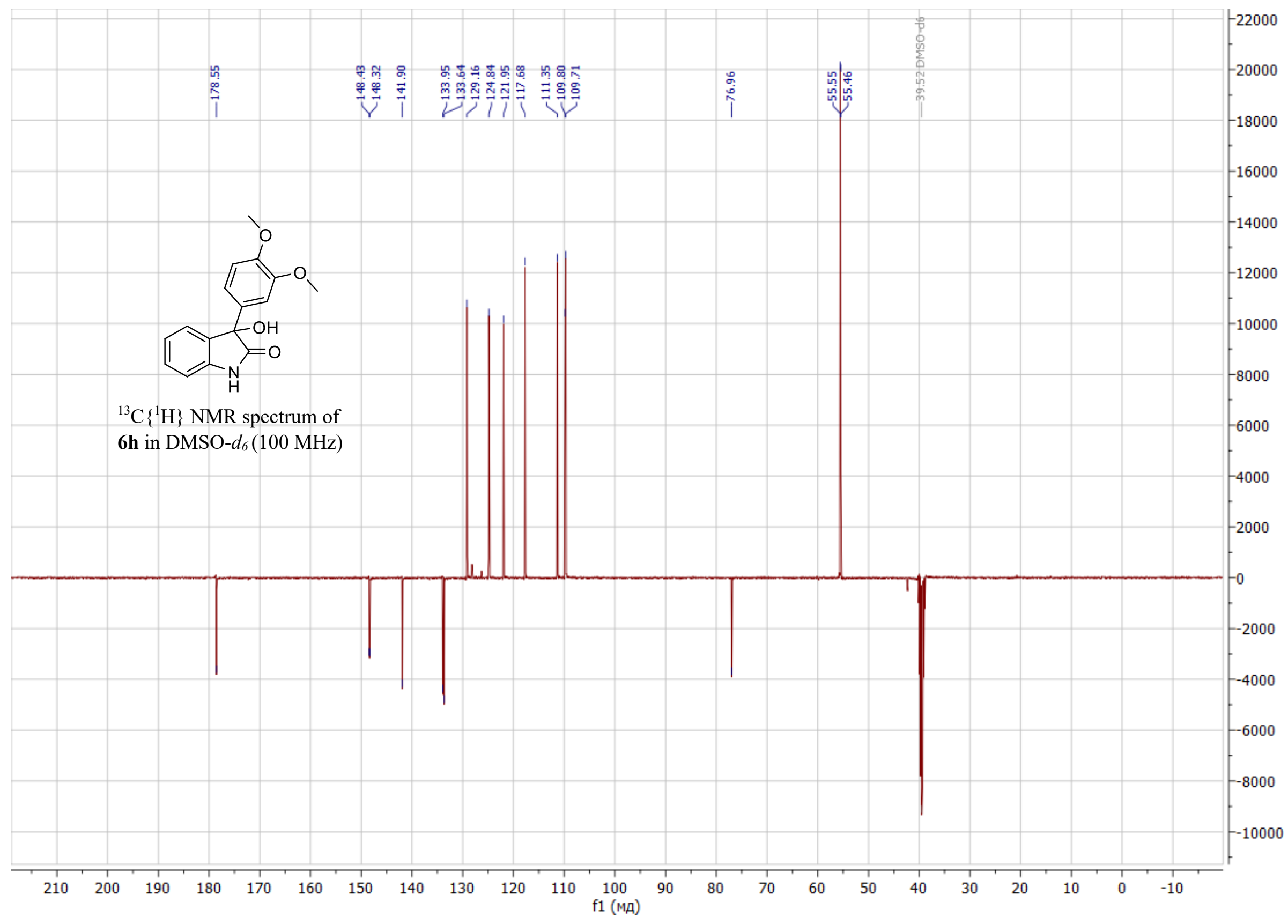




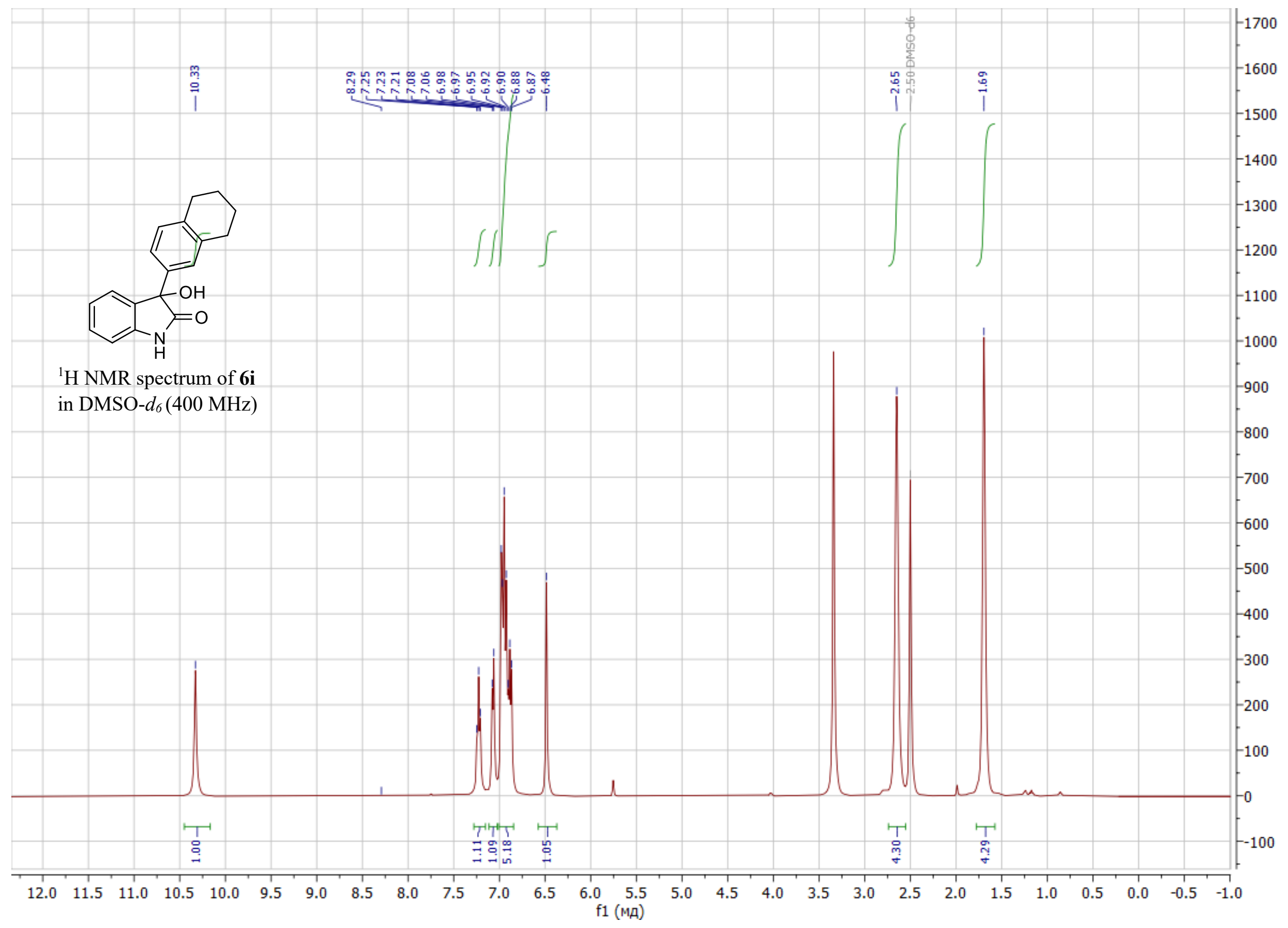




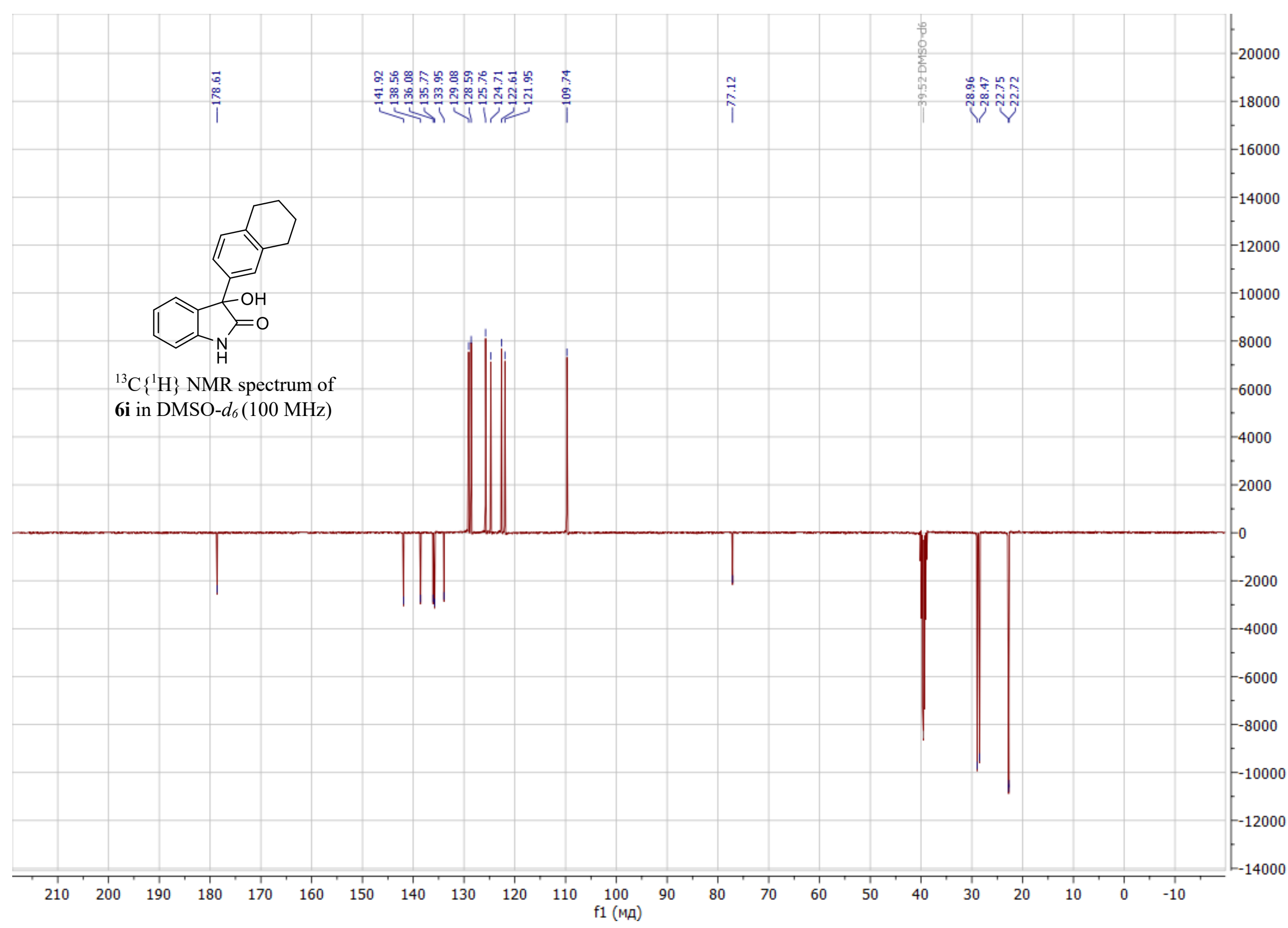




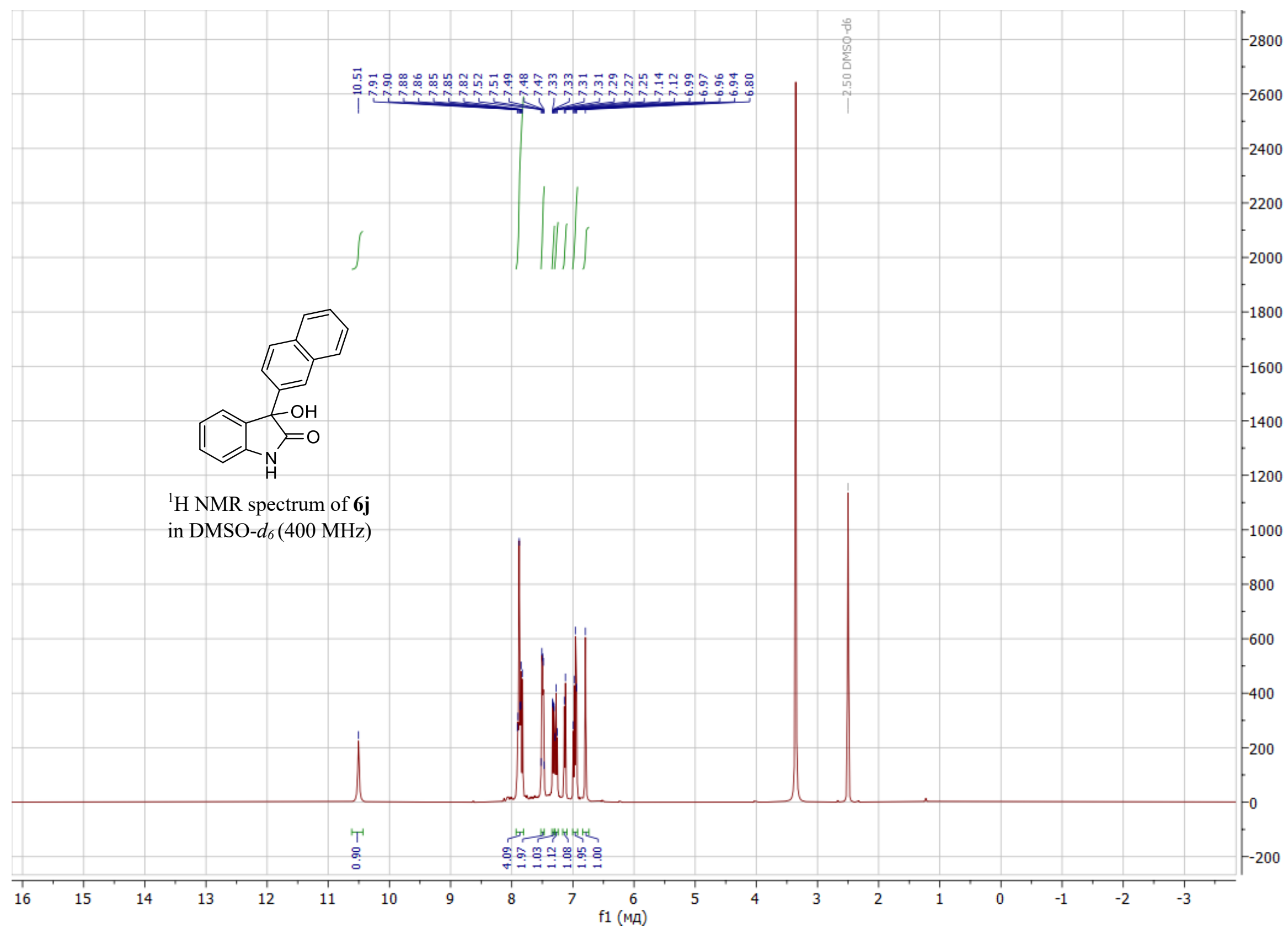




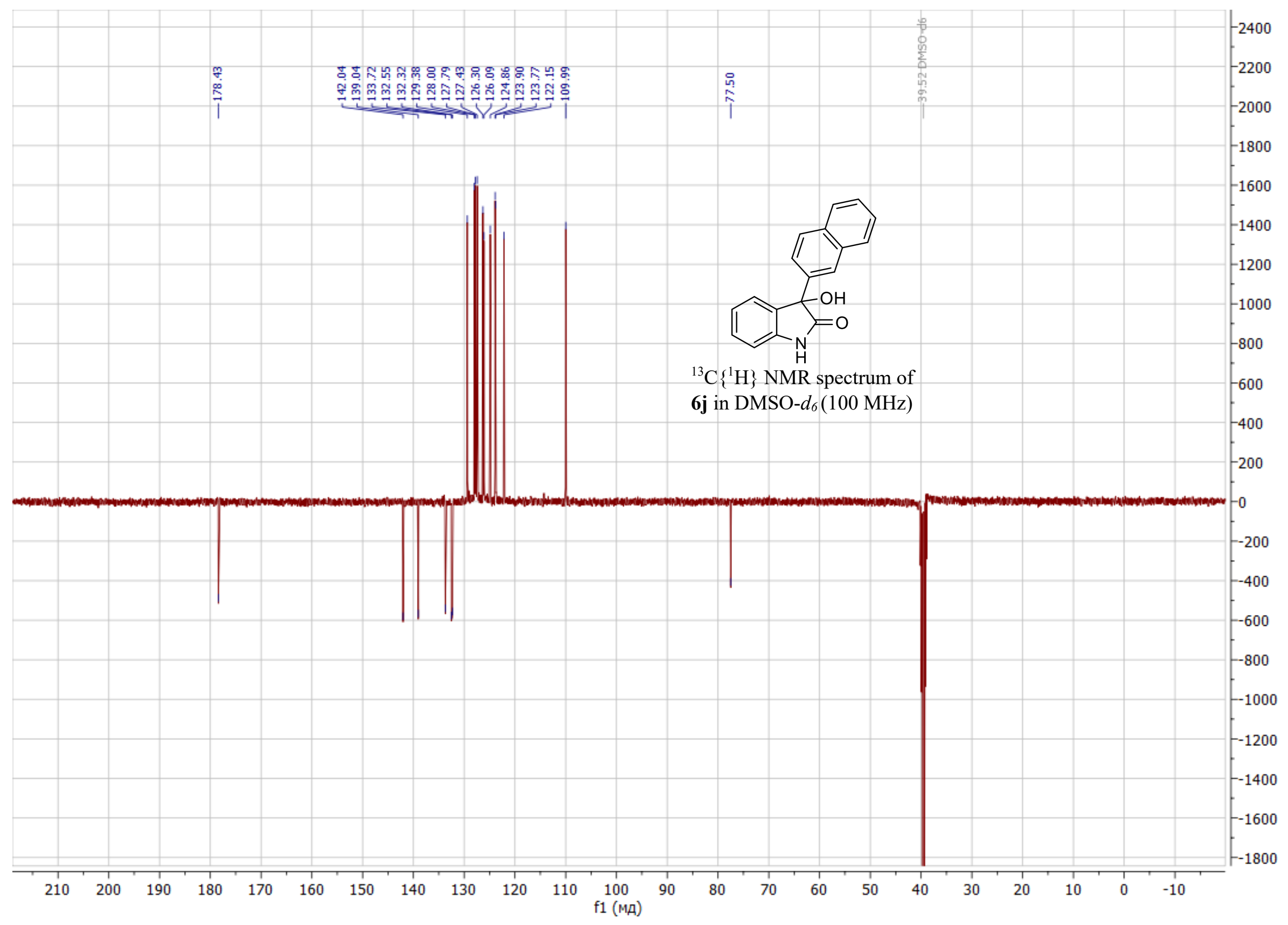




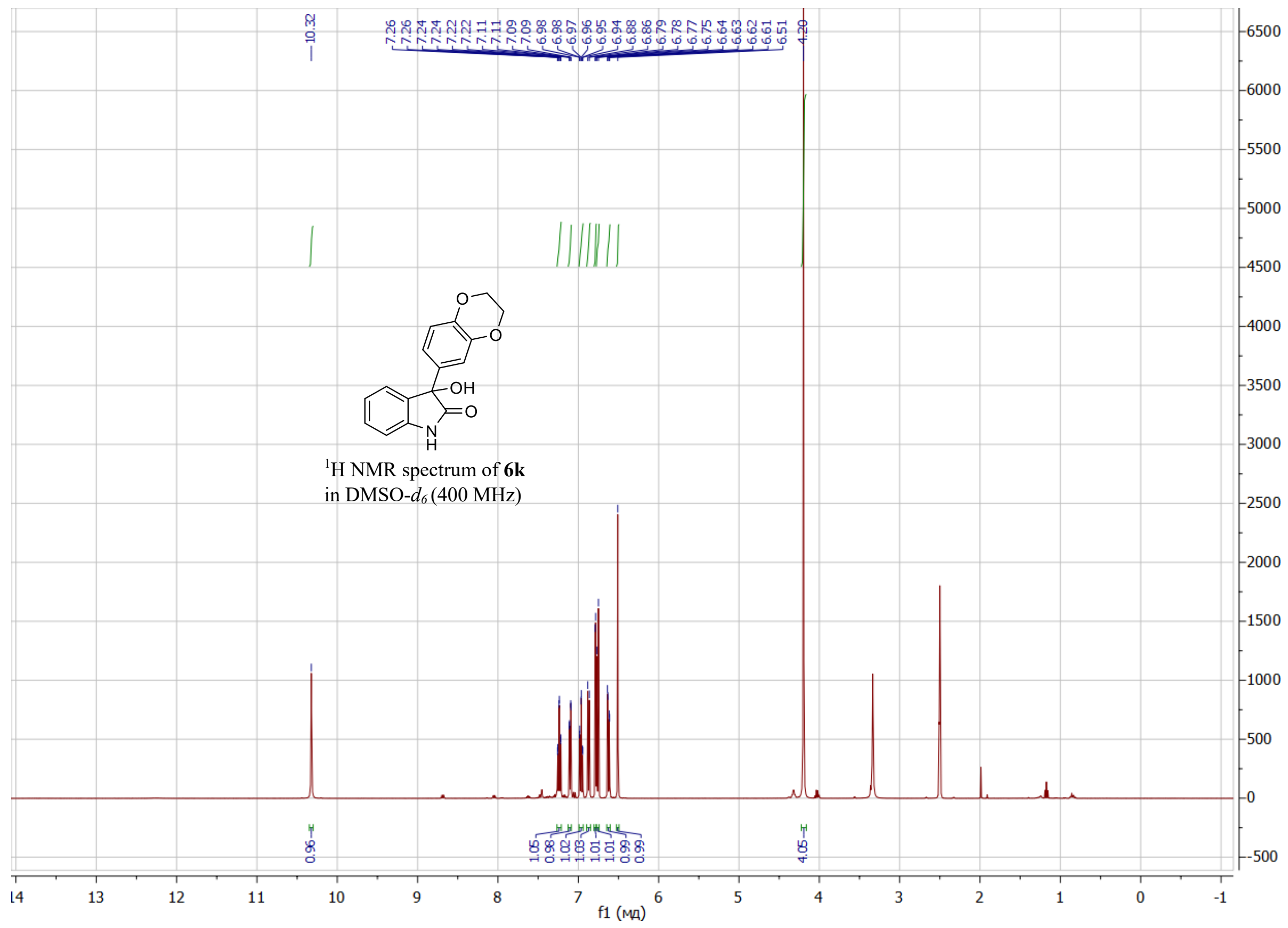




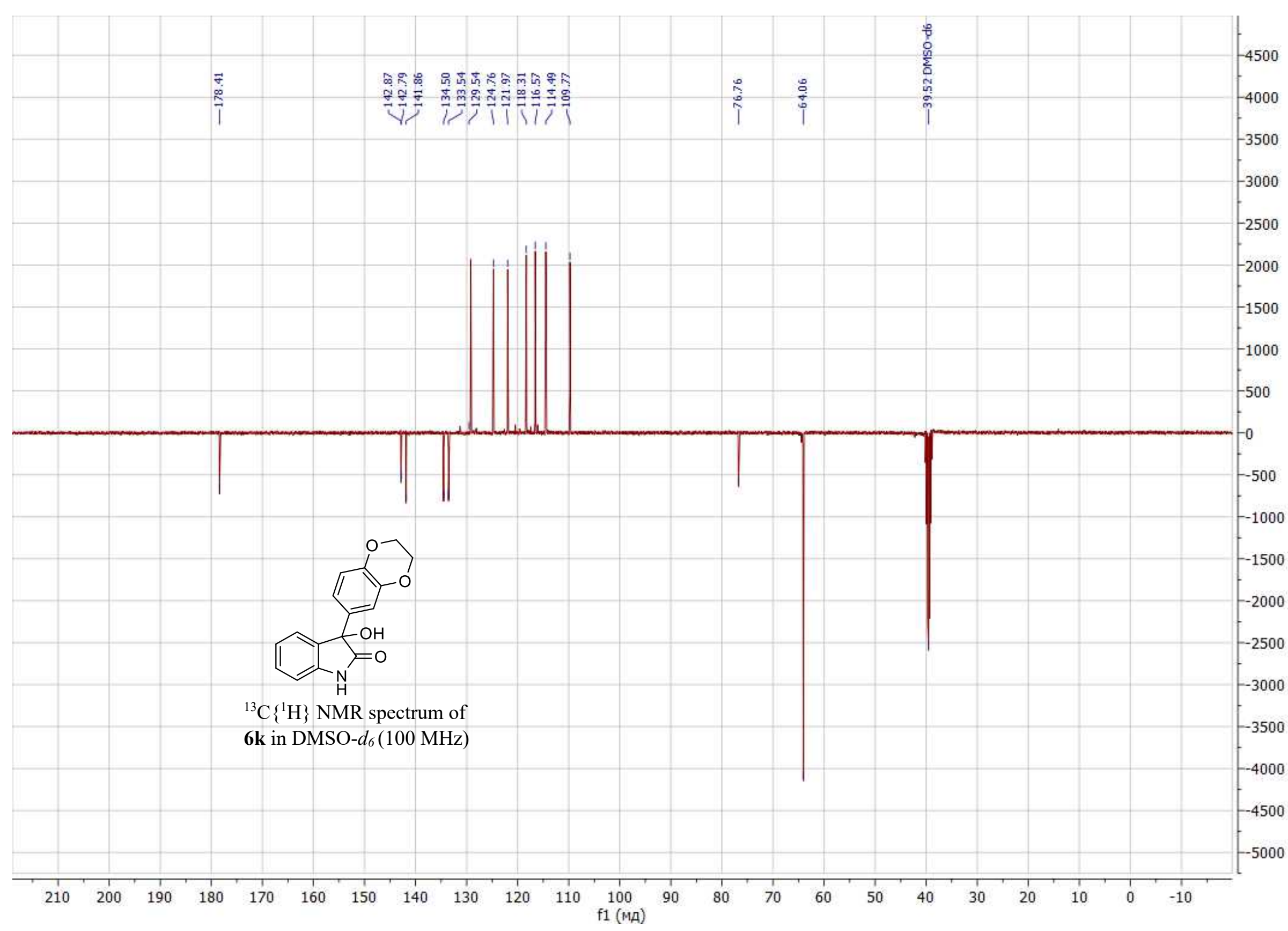




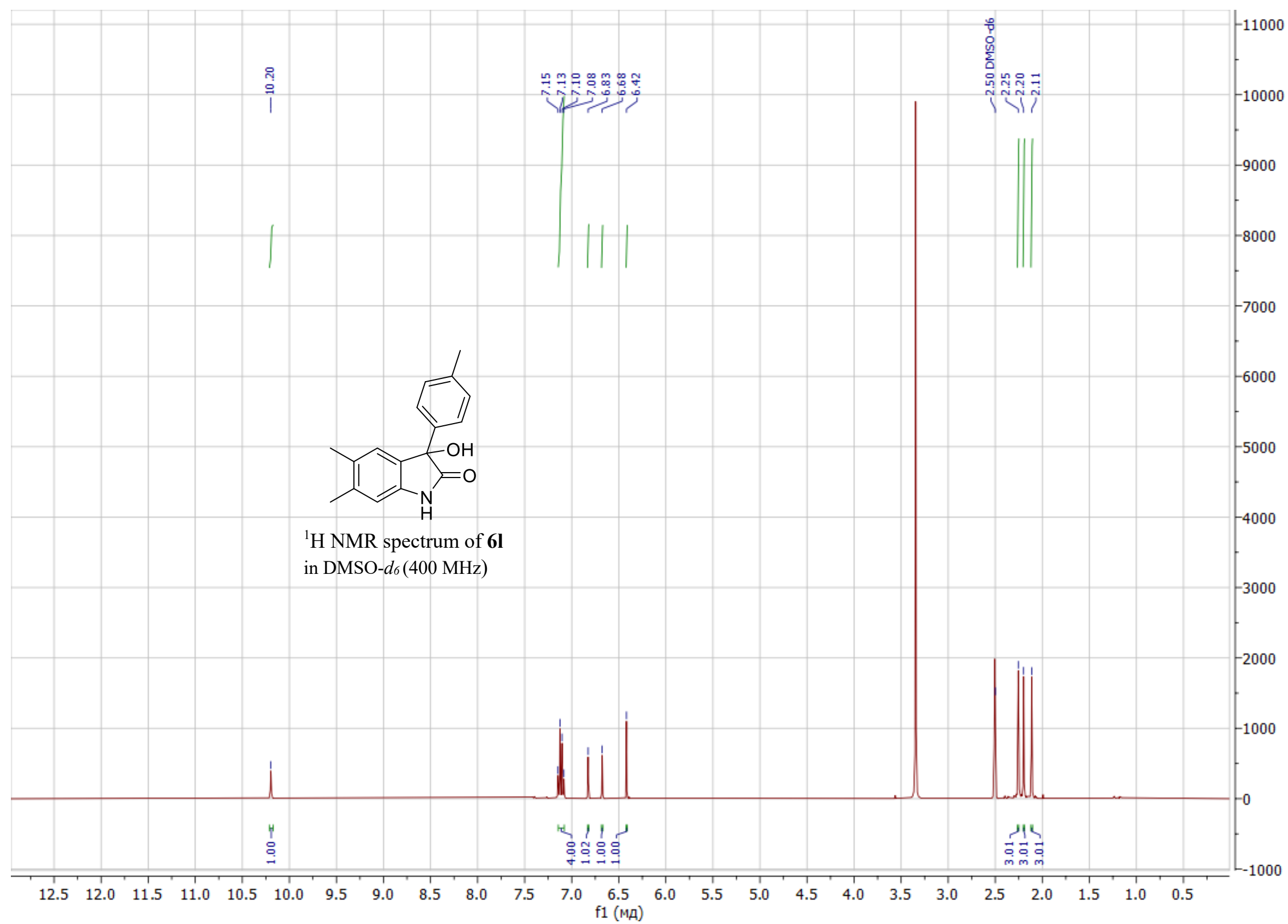




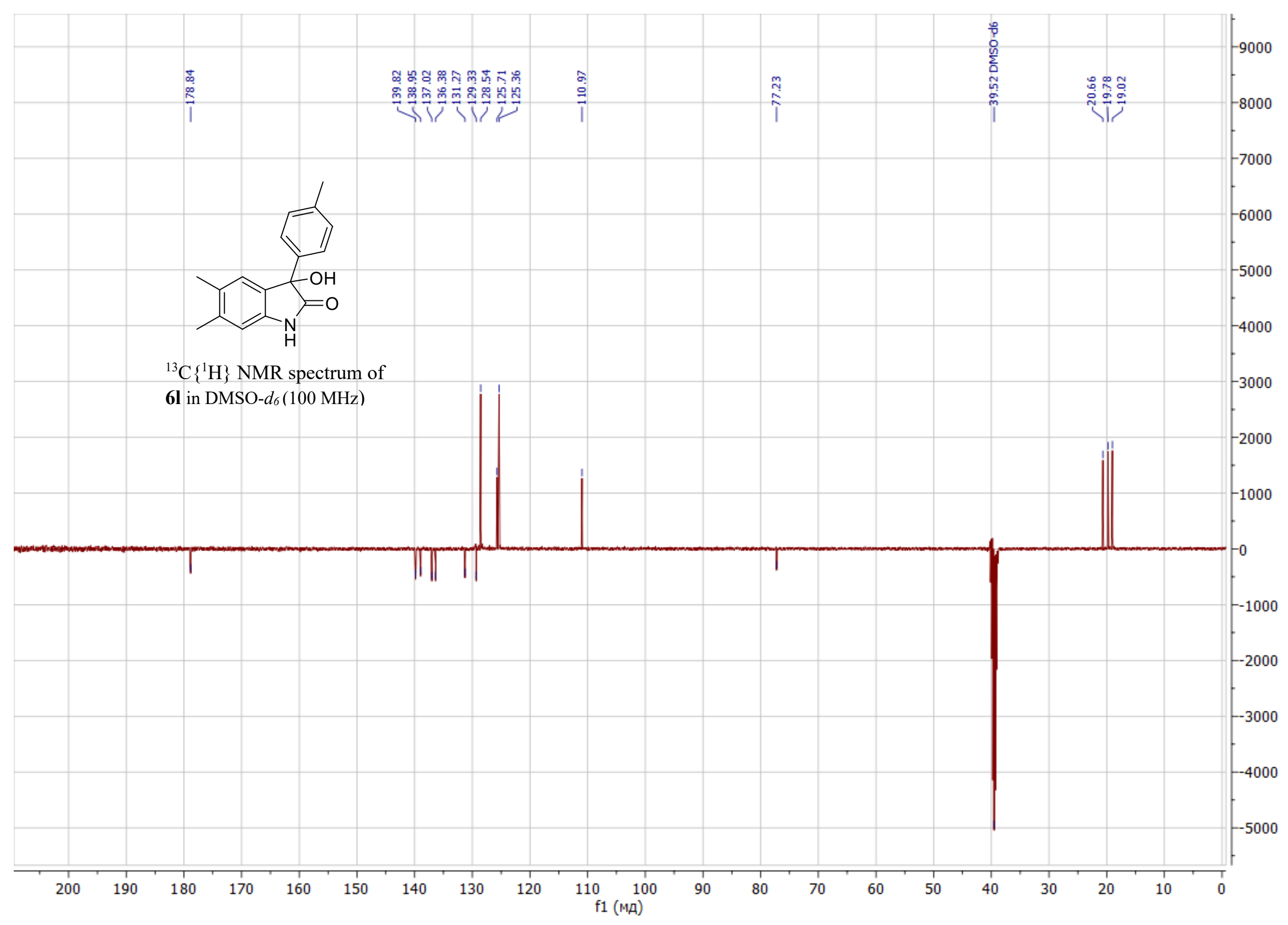




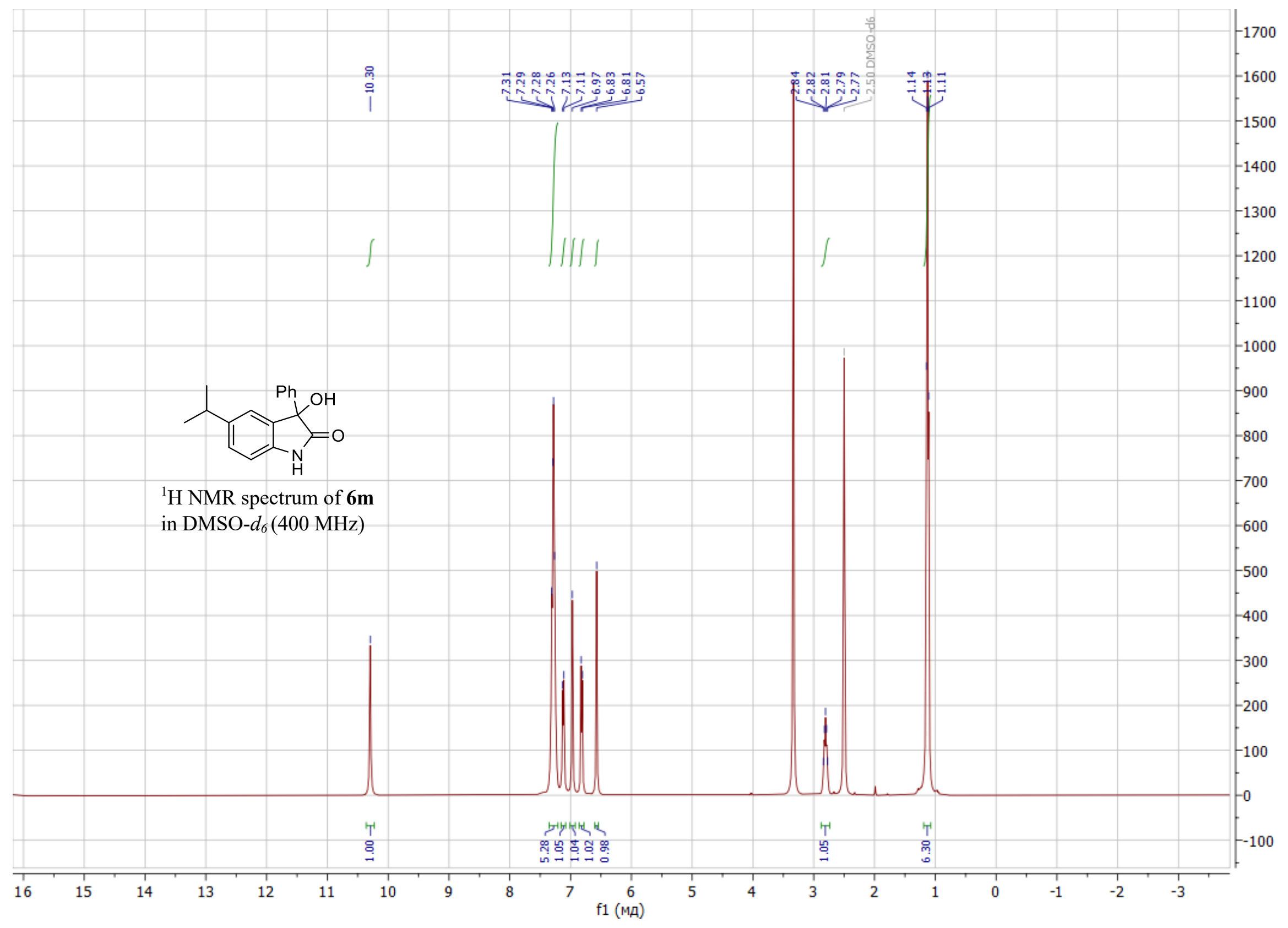




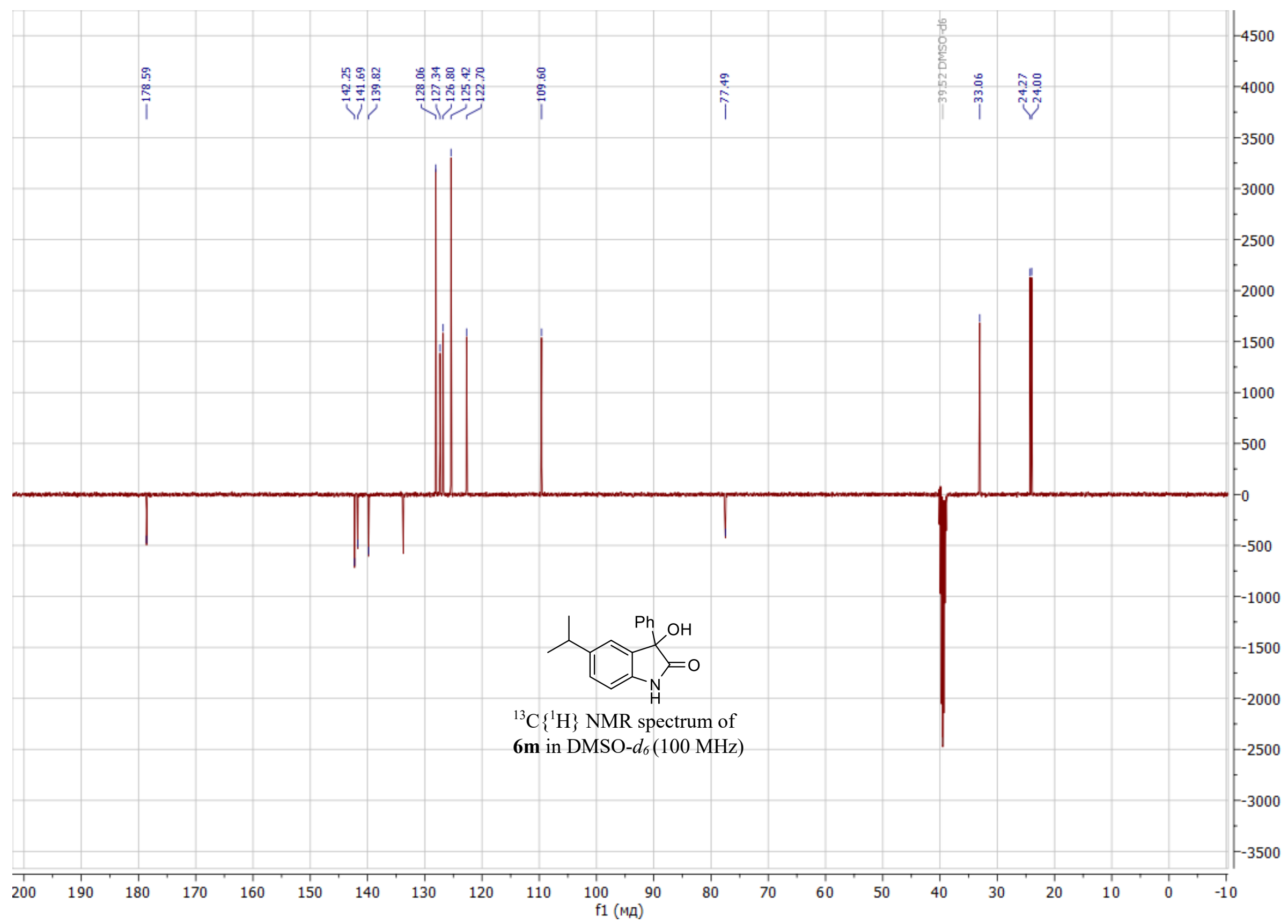




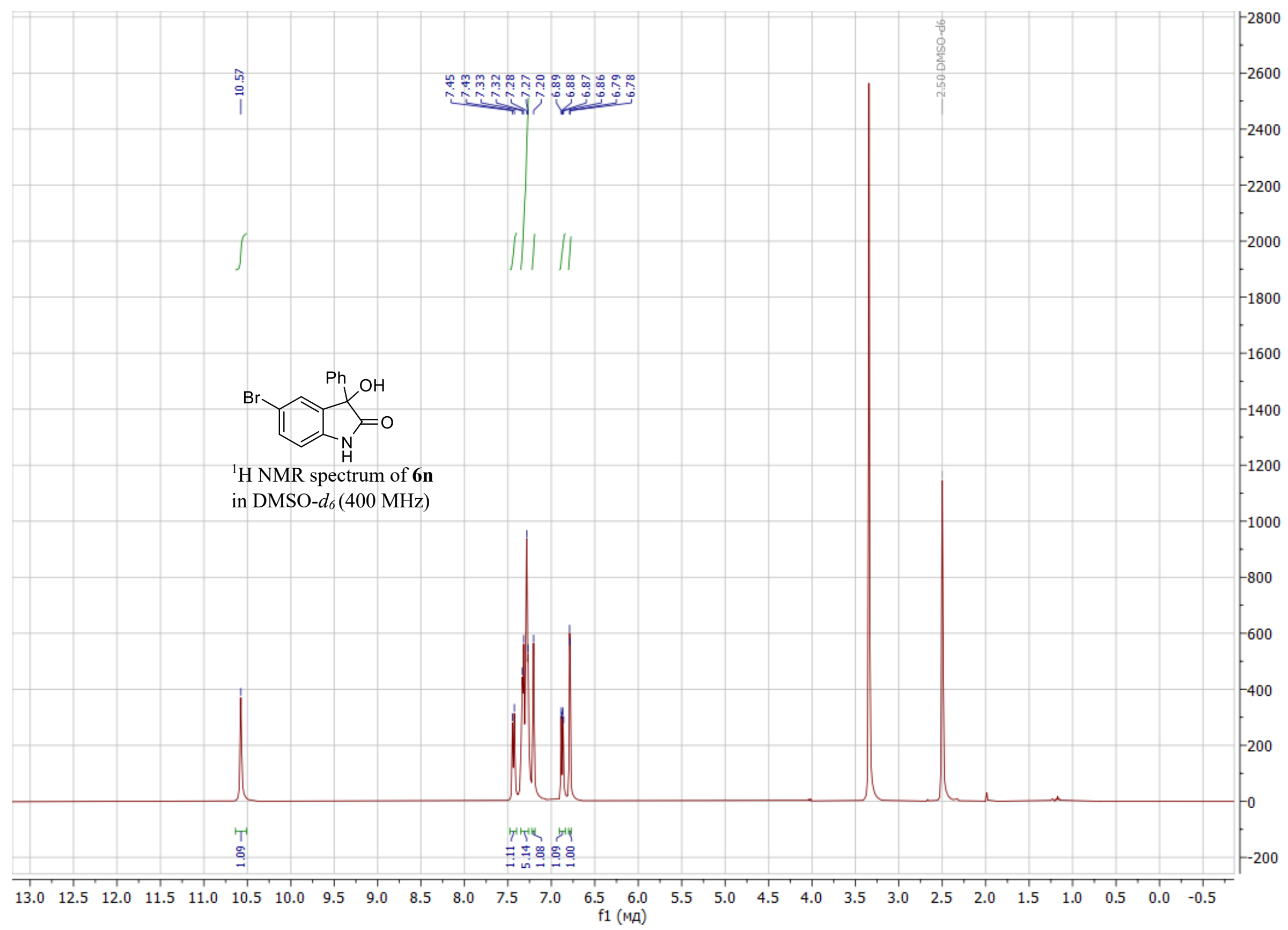




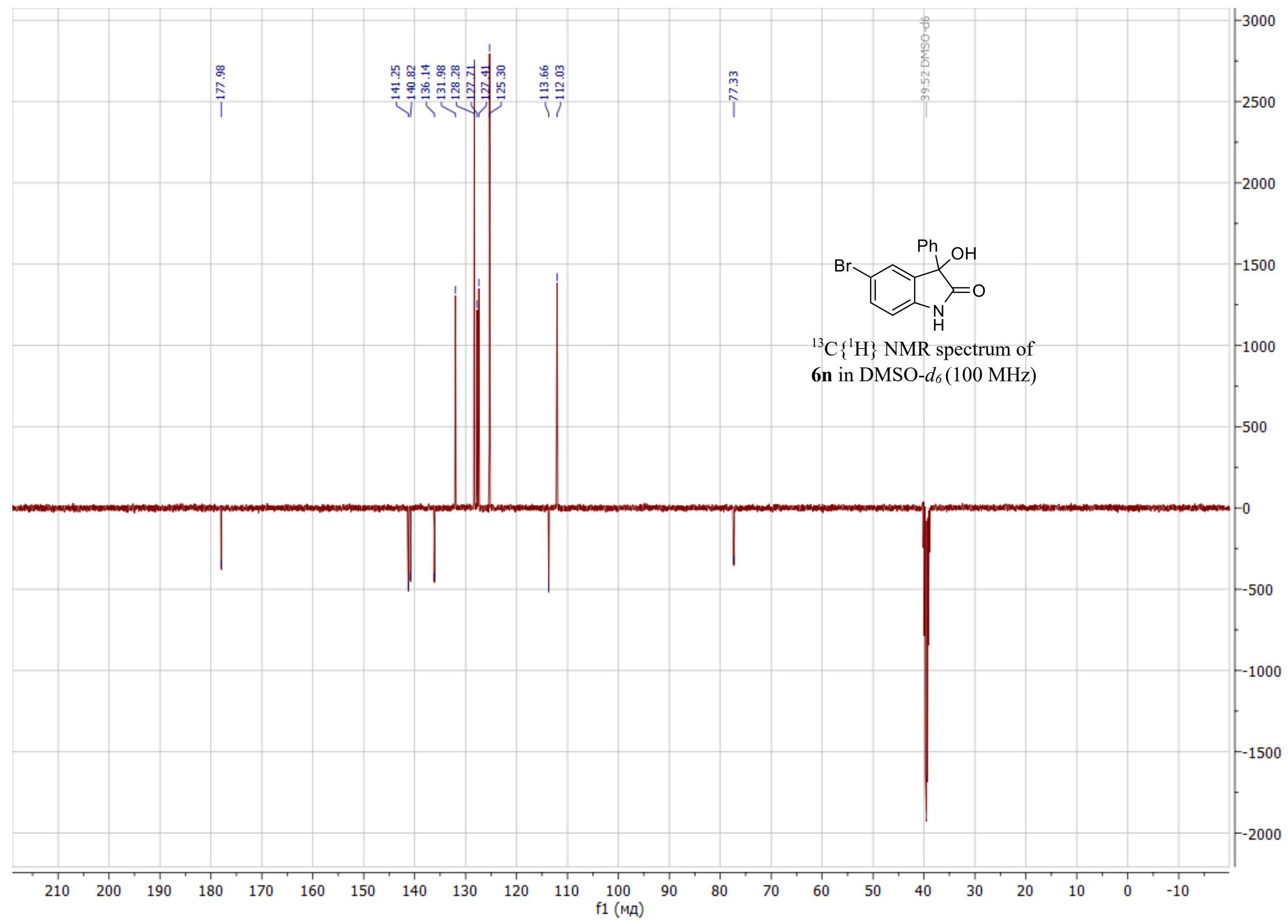




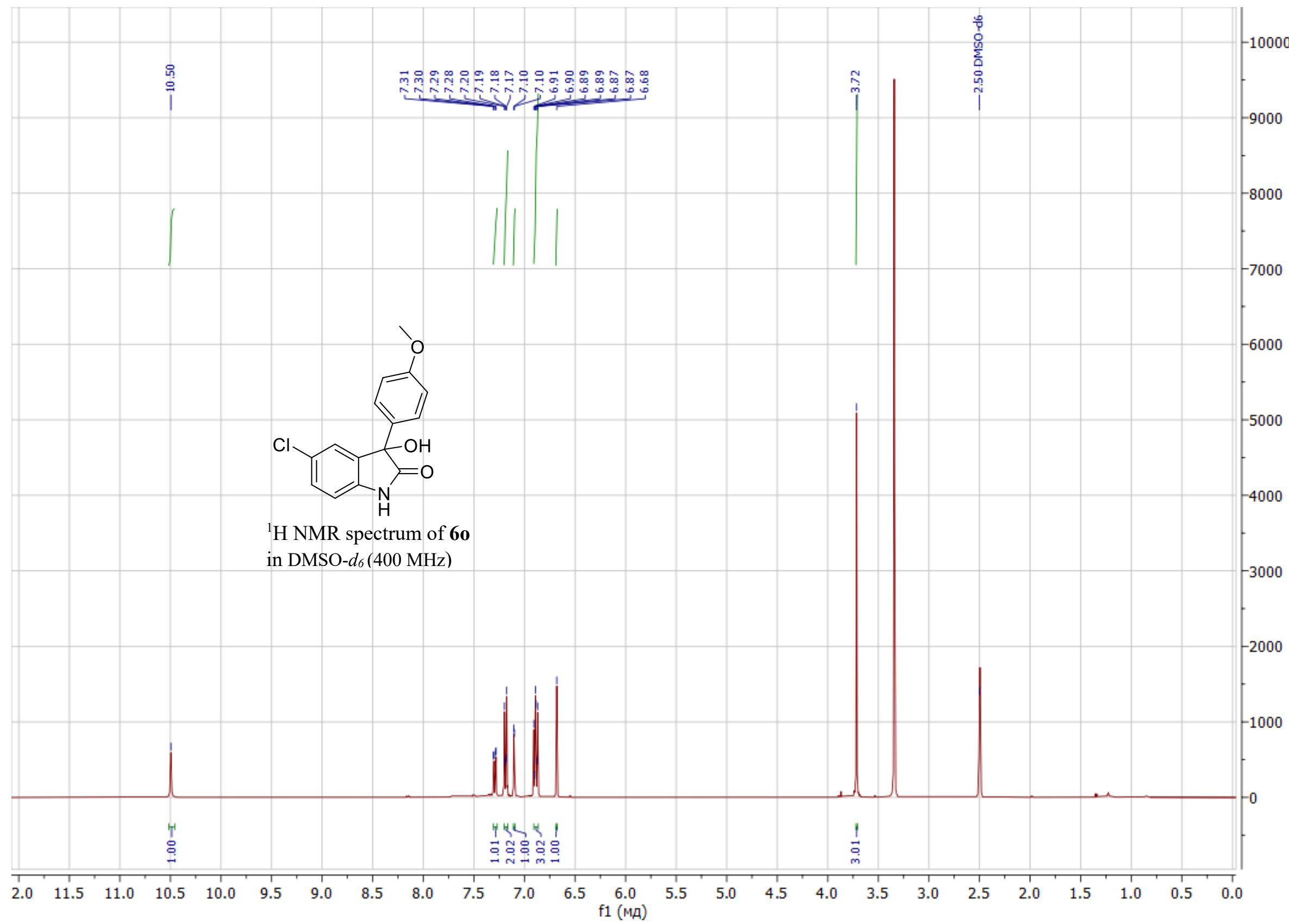




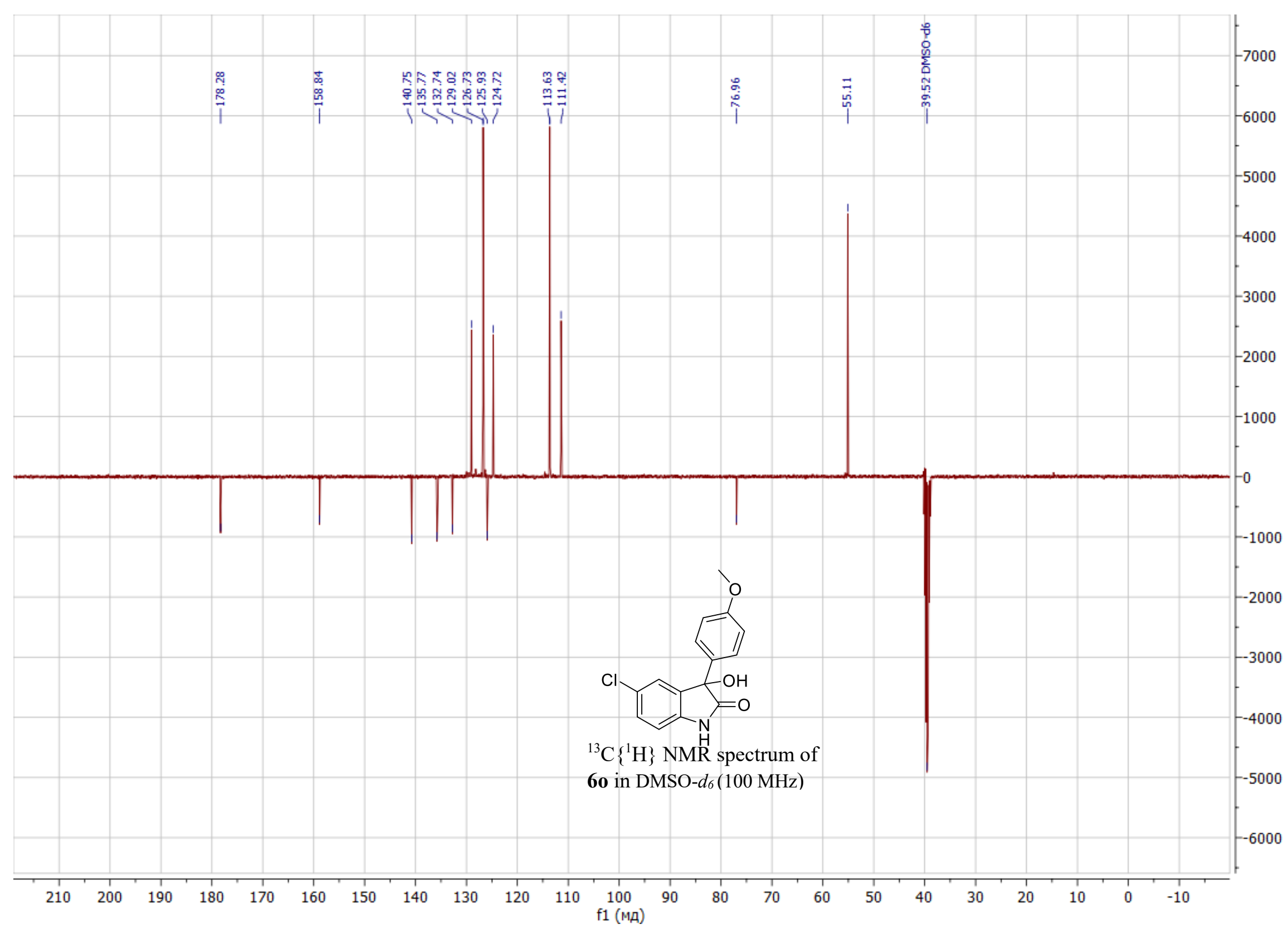


${ }^{1} \mathrm{H}$ and ${ }^{13} \mathrm{C}$ Spectral Charts for 2-benzamidobenzoic acids 13

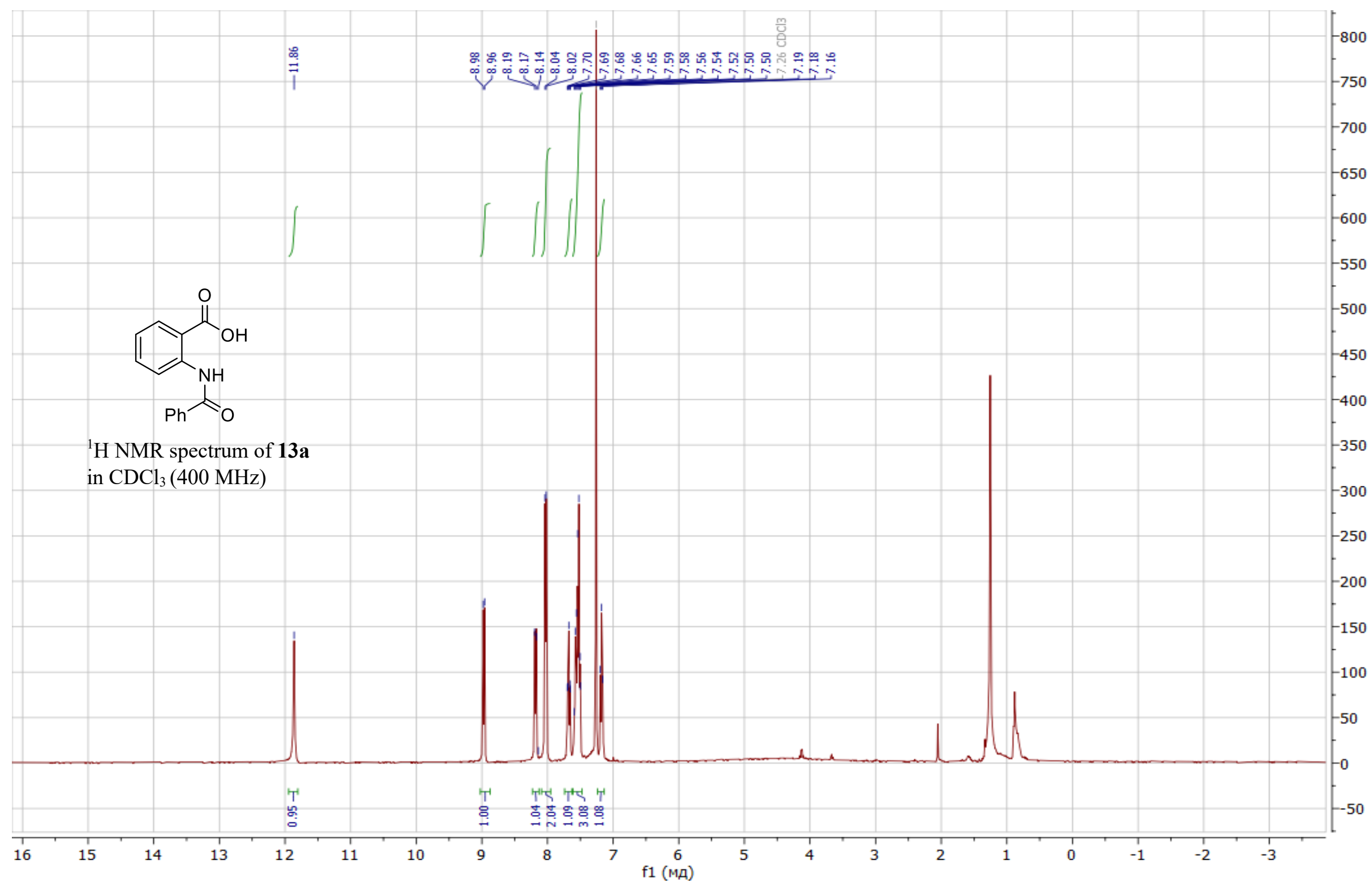




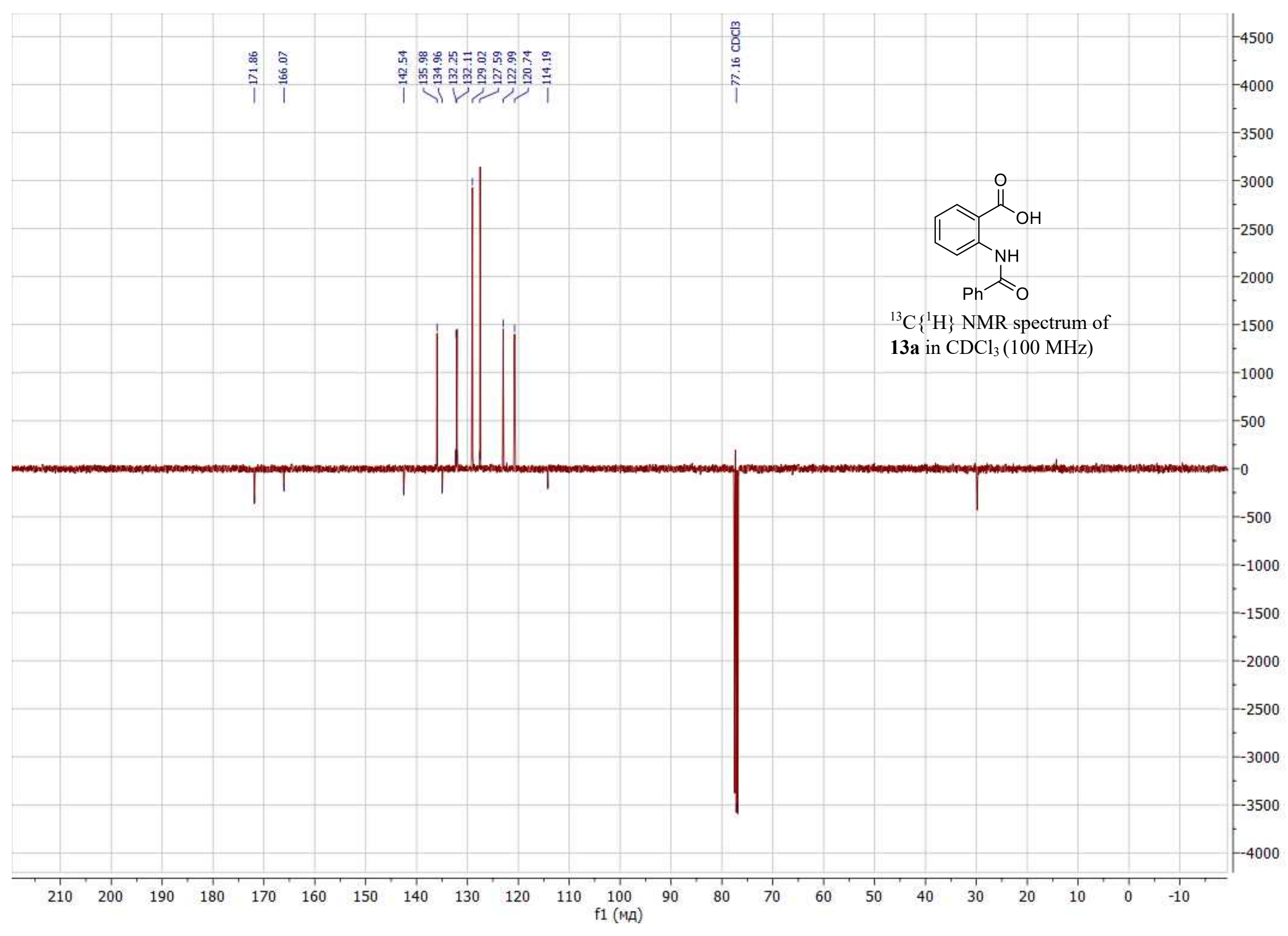




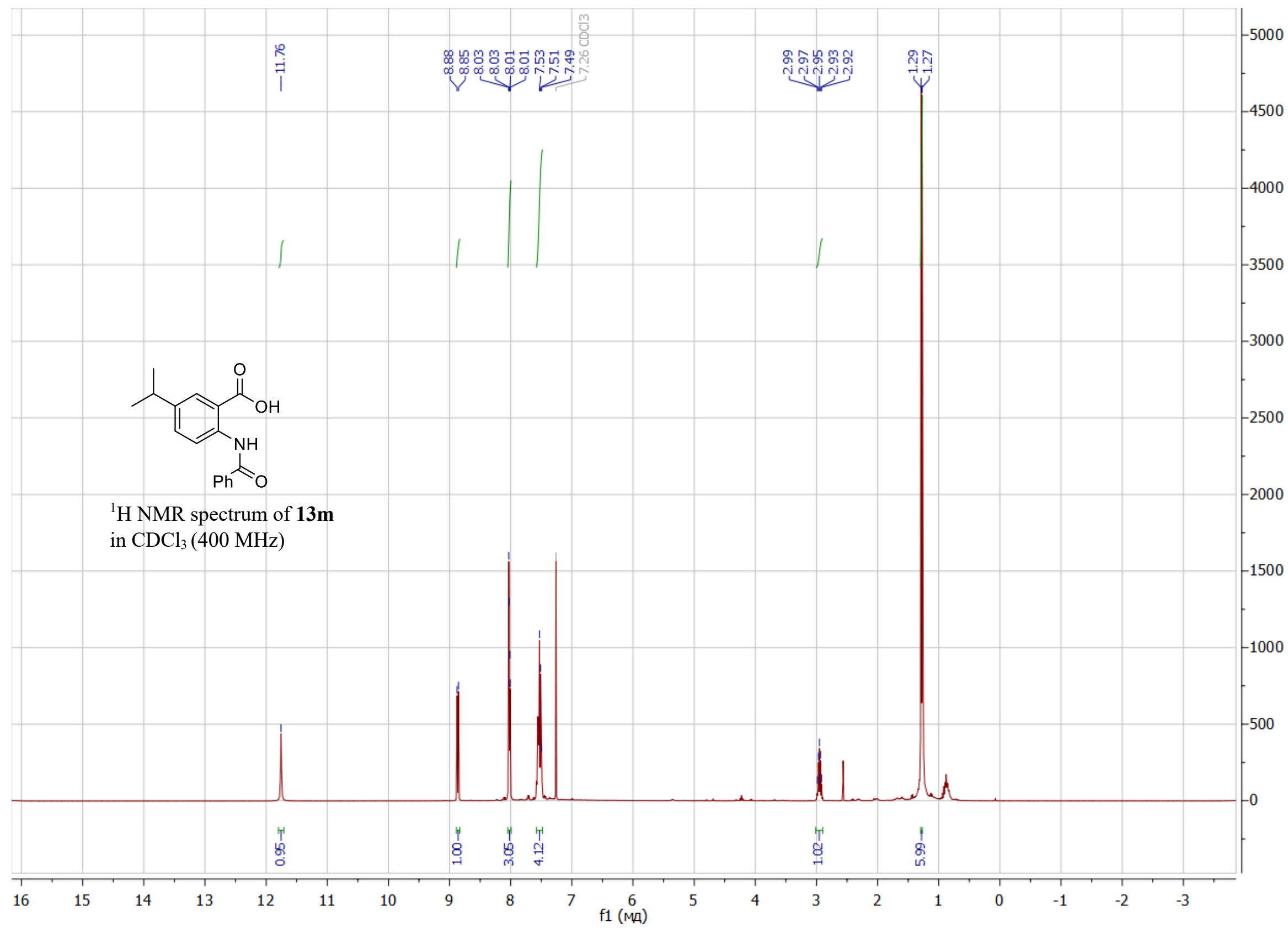




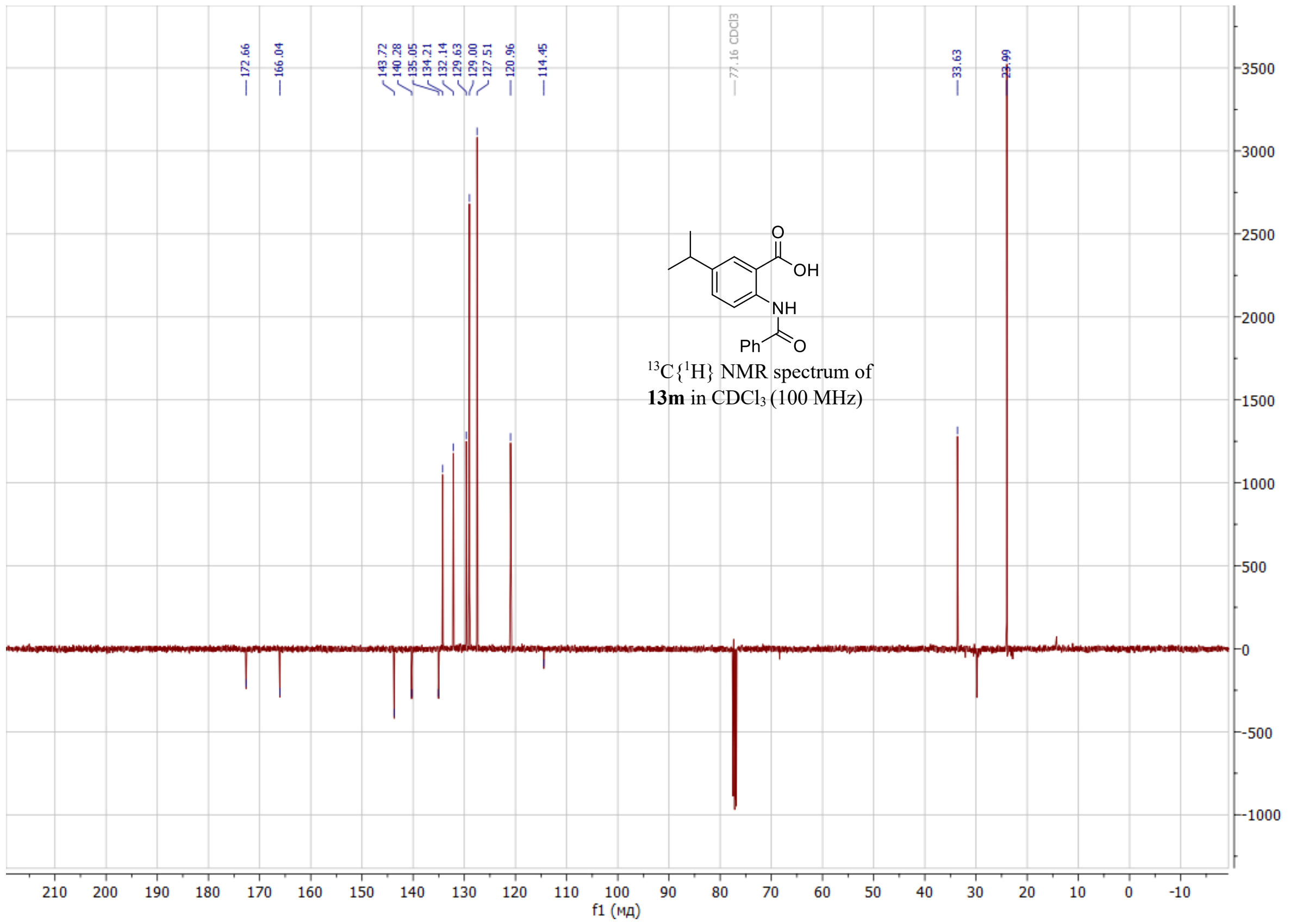

S93 


\section{HRMS Charts}

HRMS Charts for 5-isopropyl-1-methyl-2-phenyl-1H-indole (2w)

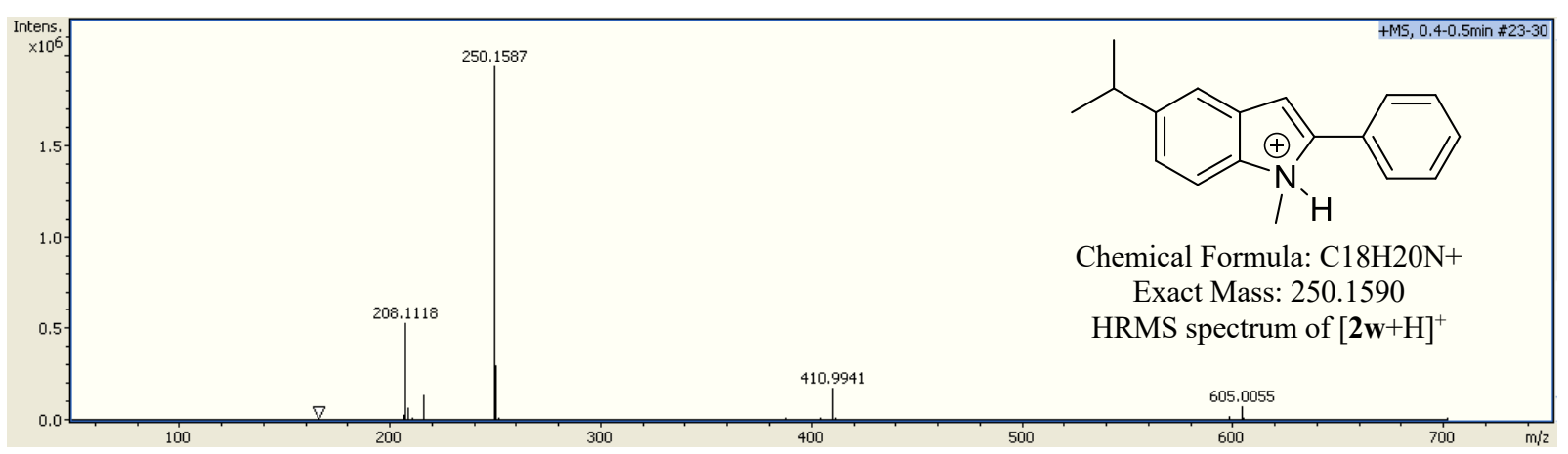


HRMS Charts for 4'H-spiro[indole-3,5'-isoxazoles] 3
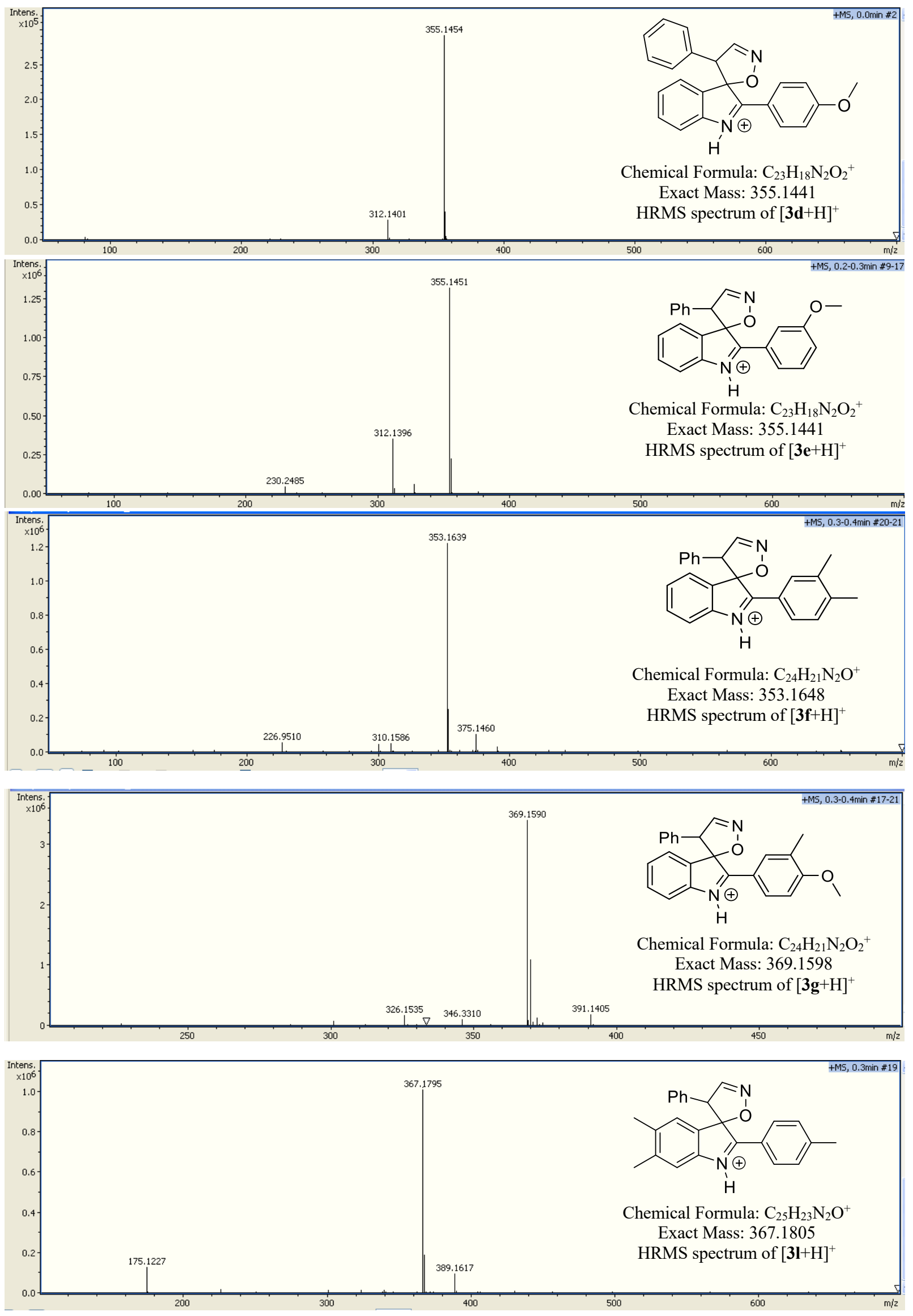

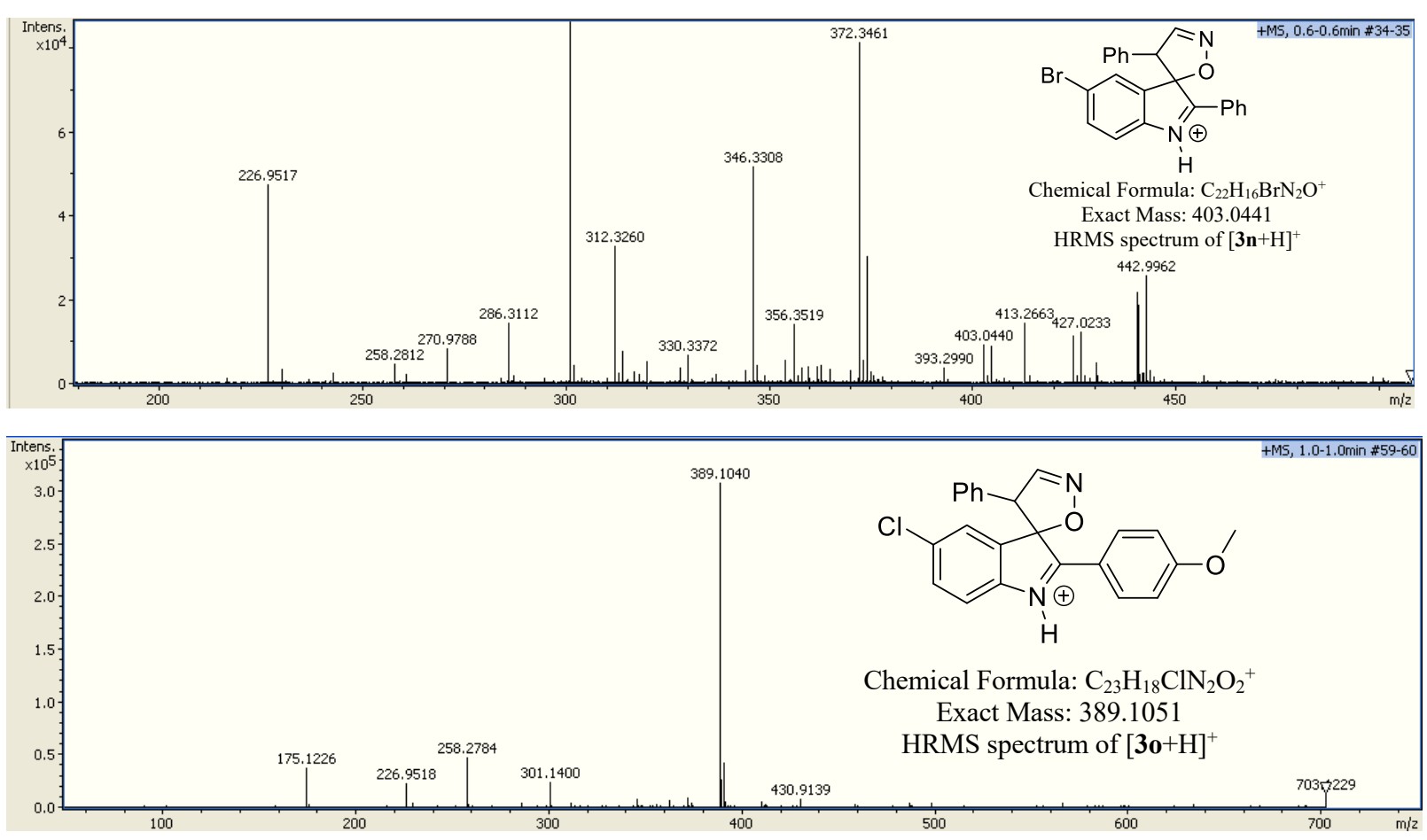

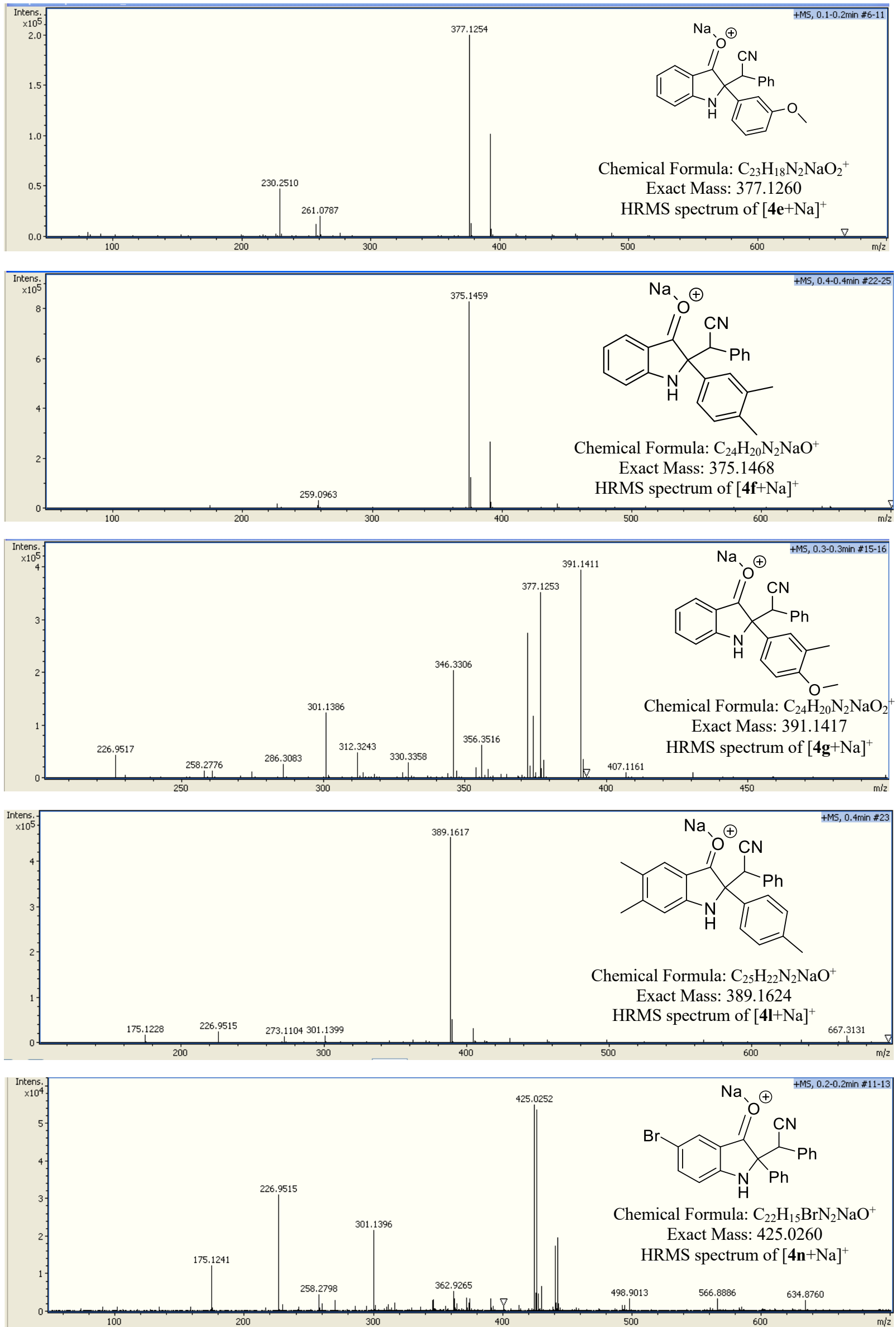

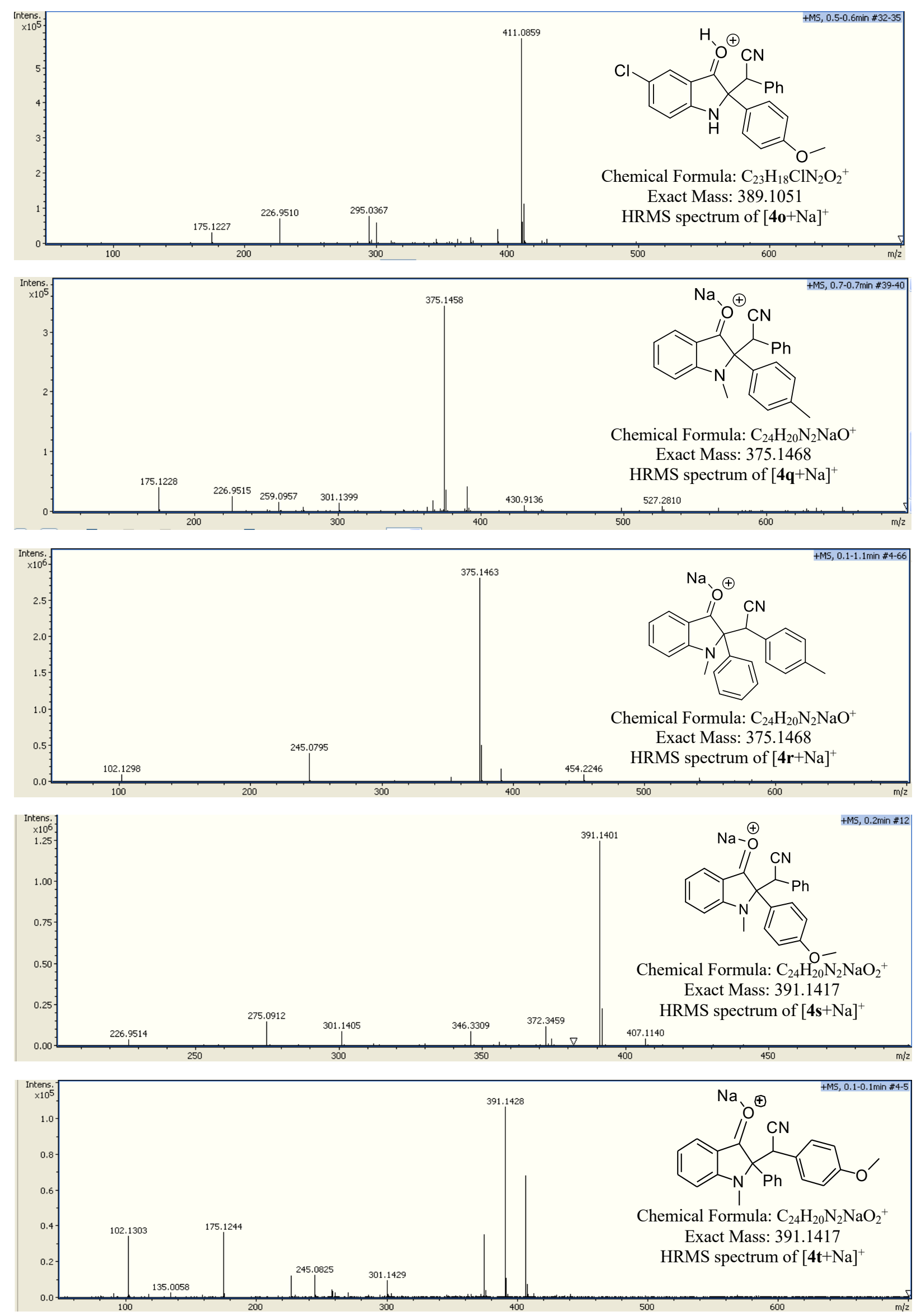

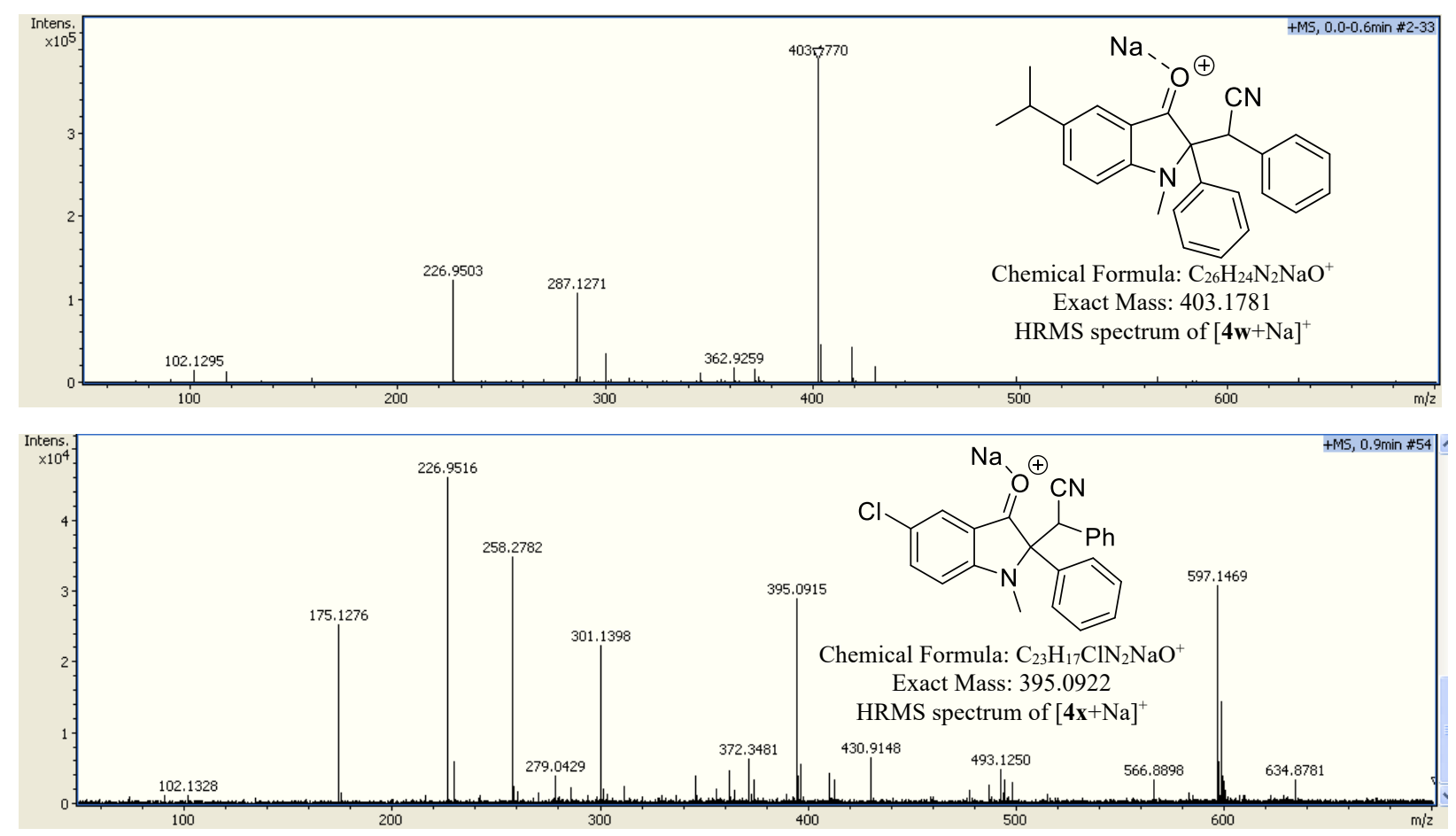
HRMS Charts for 1,3a,4,8b-tetrahydropyrrolo[3,2-b]indol-2(1H)-ones 5
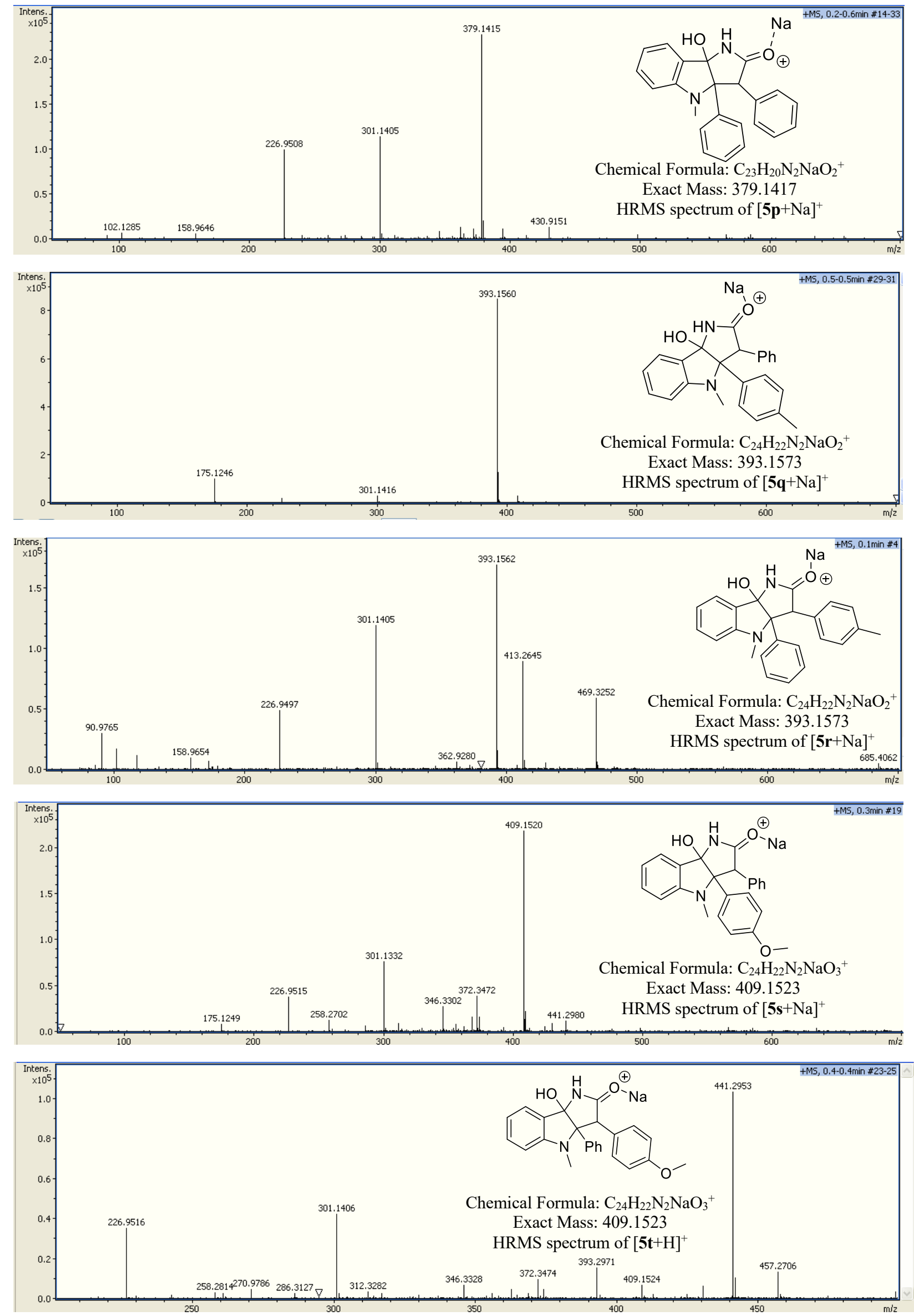

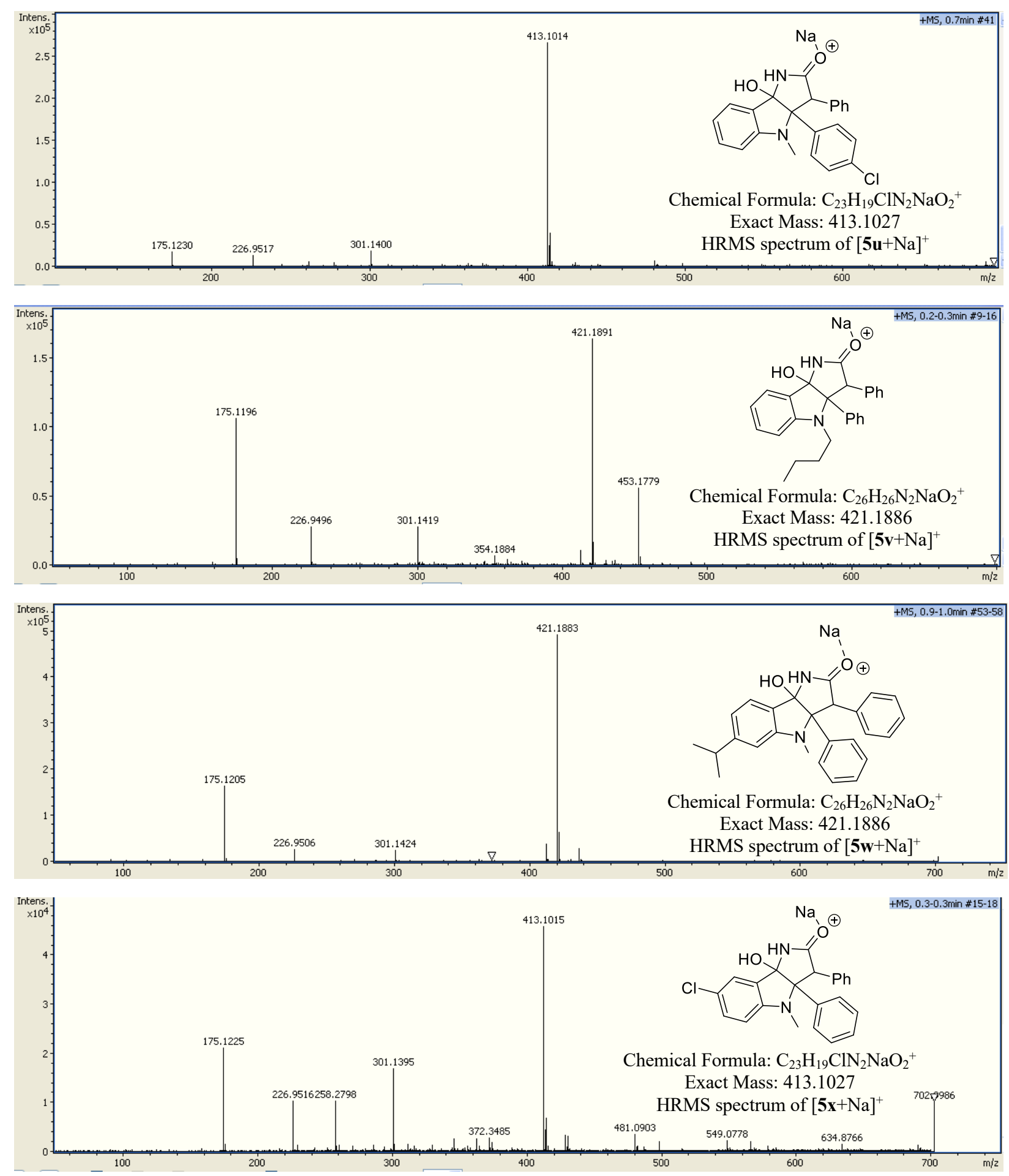

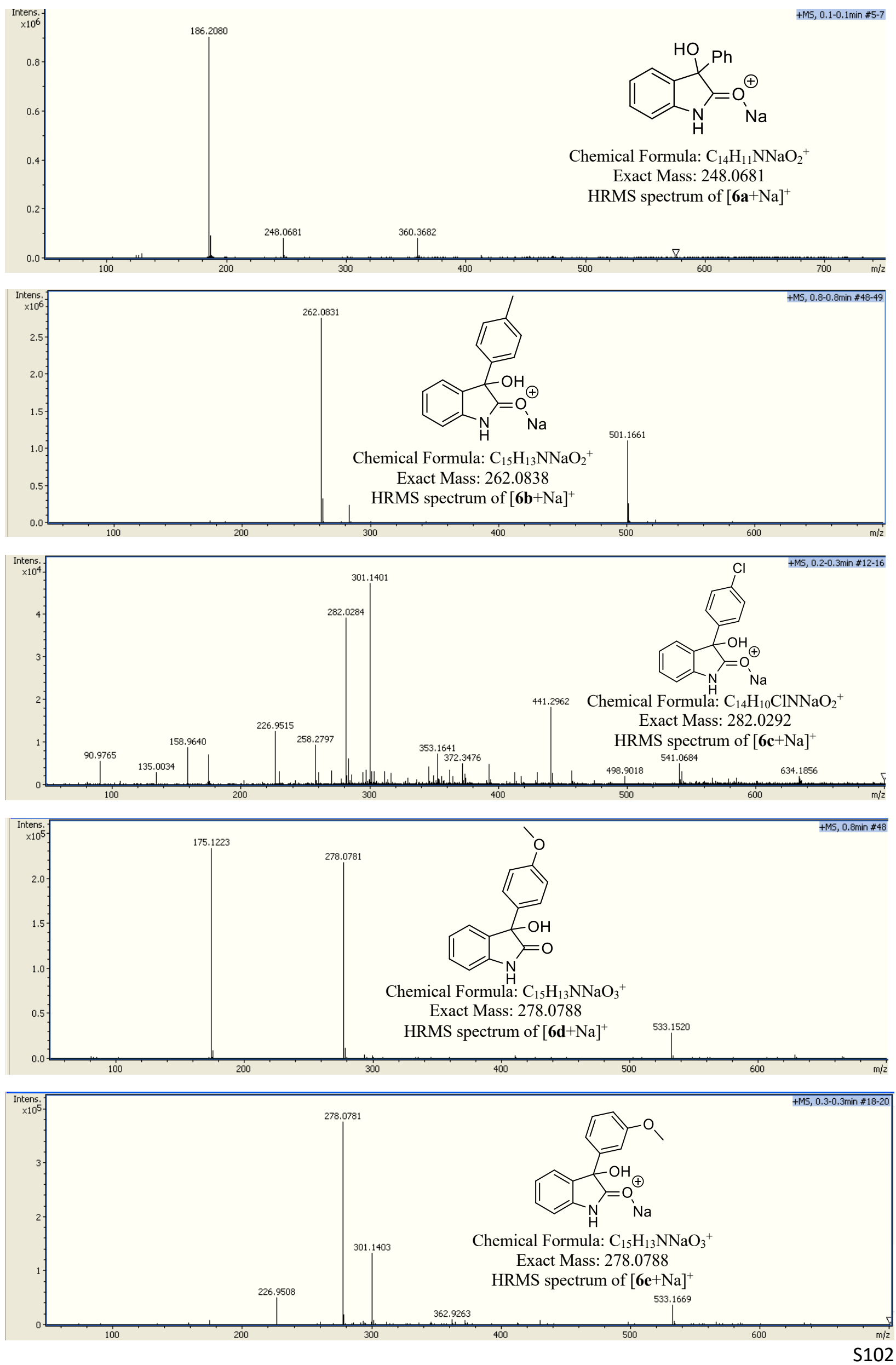

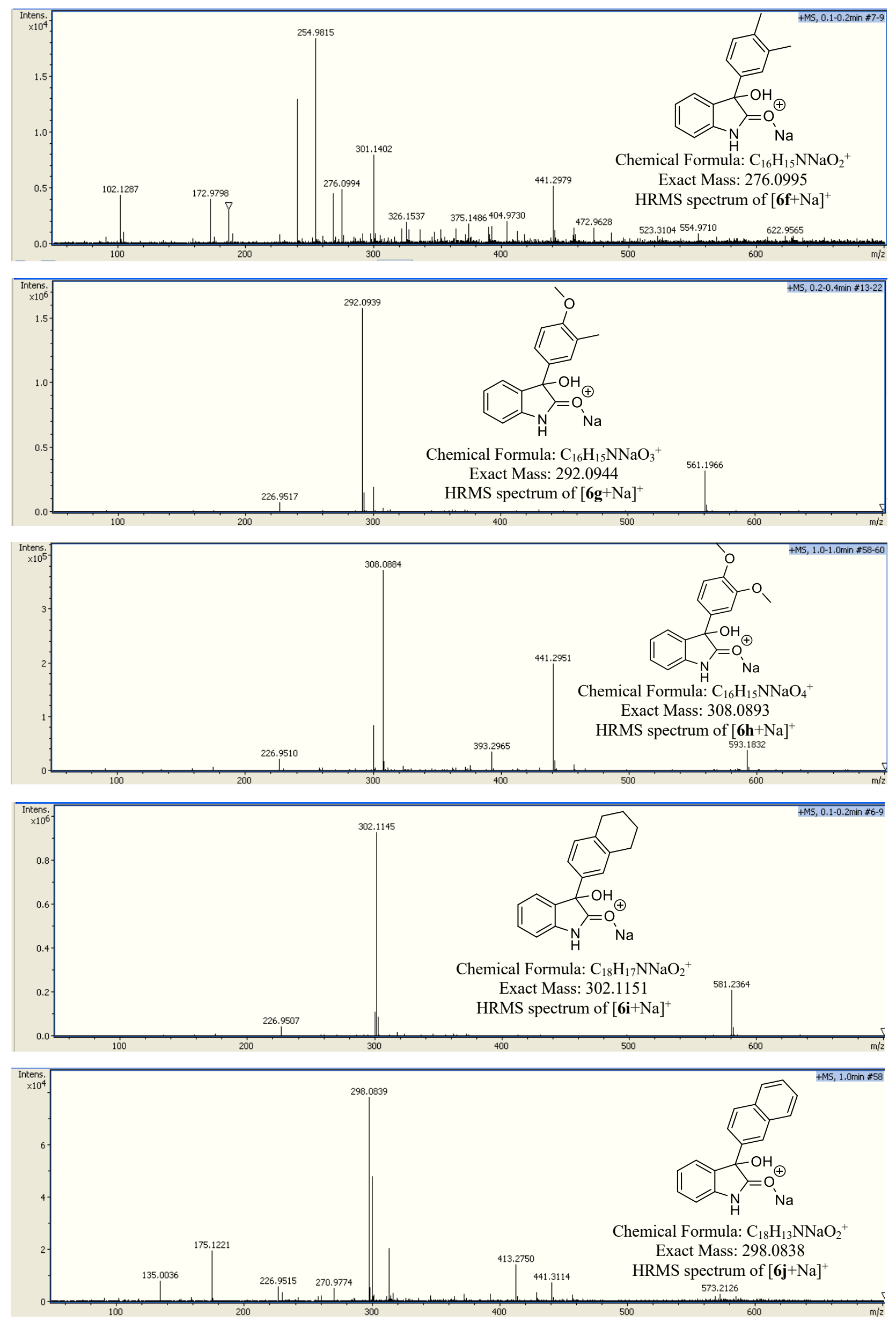

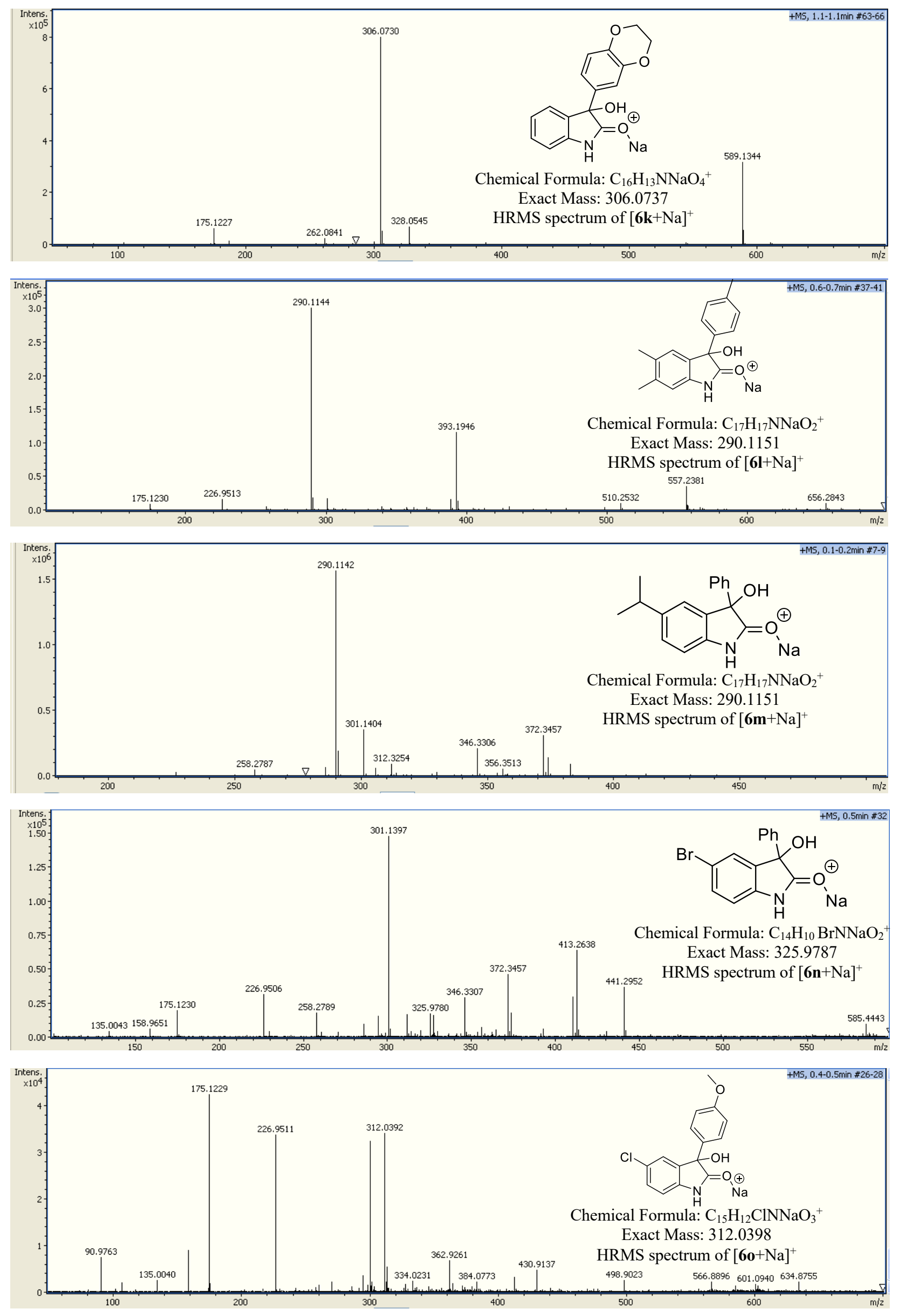


\section{X-Ray crystallography data for $5 p$}
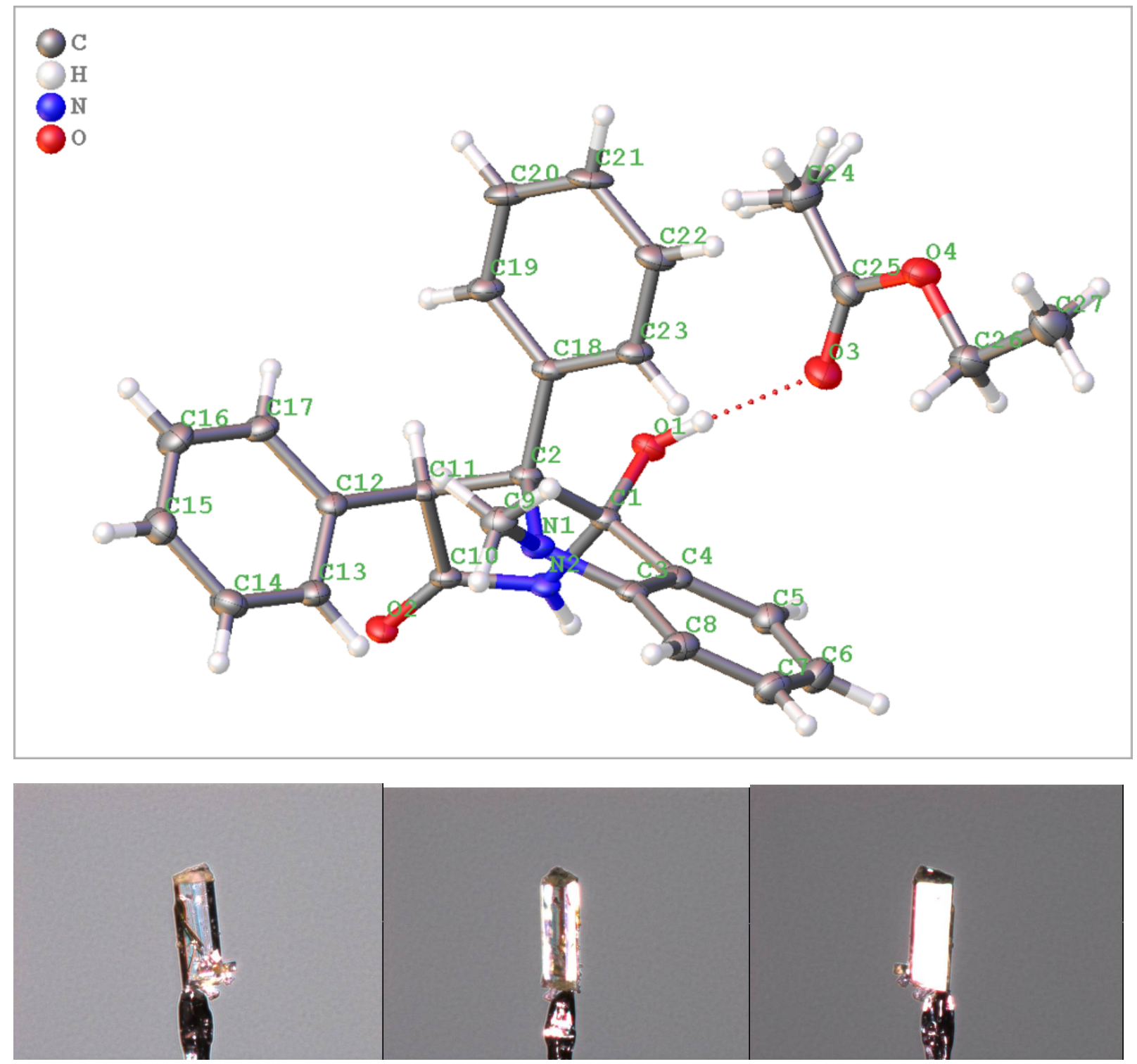

Figure S1. ORTEP drawing of the crystal structure showing 50\% probability thermal ellipsoids (top, CCDC 2105352) and microphotography of the single crystal of compound 5p used for XRay diffraction analysis at the bottom. 
Table S1 Crystal data and structure refinement for 5p.

Identification code

ANNA_ARK5XX_1

Empirical formula

$\mathrm{C}_{27} \mathrm{H}_{28} \mathrm{~N}_{2} \mathrm{O}_{4}$

Formula weight

444.51

Temperature/K

125(30)

Crystal system

Space group

triclinic

$\mathrm{a} / \AA$

P-1

$\mathrm{b} / \AA$

$8.9958(3)$

$\mathrm{c} / \AA$

$11.7405(6)$

$12.0993(5)$

$\alpha /{ }^{\circ}$

67.721(4)

$\beta /{ }^{\circ}$

$79.125(3)$

$\gamma /{ }^{\circ}$

$88.176(3)$

Volume $/ \AA^{3}$

1160.13(9)

Z

2

$\rho_{\text {calc }} / \mathrm{cm}^{3}$

1.272

$\mu / \mathrm{mm}^{-1}$

0.691

$\mathrm{F}(000)$

472.0

Crystal size $/ \mathrm{mm}^{3}$

$0.408 \times 0.153 \times 0.116$

Radiation

$\mathrm{Cu} \mathrm{K \alpha}(\lambda=1.54184)$

$2 \Theta$ range for data collection $/{ }^{\circ} 8.044$ to 161.988

Index ranges

$-8 \leq \mathrm{h} \leq 11,-15 \leq \mathrm{k} \leq 14,-15 \leq 1 \leq 15$

Reflections collected

23047

Independent reflections

$4868\left[\mathrm{R}_{\text {int }}=0.0599, \mathrm{R}_{\text {sigma }}=0.0334\right]$

Data/restraints/parameters

$4868 / 0 / 333$

Goodness-of-fit on $\mathrm{F}^{2}$

1.052

Final $R$ indexes $[\mathrm{I}>=2 \sigma(\mathrm{I})] \quad \mathrm{R}_{1}=0.0469, \mathrm{wR}_{2}=0.1254$

Final $\mathrm{R}$ indexes [all data] $\quad \mathrm{R}_{1}=0.0520, \mathrm{wR}_{2}=0.1314$

Largest diff. peak/hole / e $\AA^{-3} 0.41 /-0.26$ 
Table S2 Fractional Atomic Coordinates $\left(\times 10^{4}\right)$ and Equivalent Isotropic Displacement Parameters $\left(\AA^{2} \times 10^{3}\right)$ for $5 \mathrm{p} . \mathrm{U}_{\text {eq }}$ is defined as $1 / 3$ of of the trace of the orthogonalised UIJ tensor.

\begin{tabular}{|c|c|c|c|c|}
\hline Atom & $x$ & $y$ & $z$ & $\mathbf{U}(\mathbf{e q})$ \\
\hline $\mathrm{O} 1$ & $5902.8(10)$ & $4835.2(9)$ & $1888.0(9)$ & $19.6(2)$ \\
\hline $\mathrm{O} 2$ & $9339.3(10)$ & $4180.9(10)$ & $-820.6(9)$ & $20.9(2)$ \\
\hline $\mathrm{O} 4$ & $1984.0(11)$ & $3805.0(11)$ & $5529.4(10)$ & $27.3(3)$ \\
\hline $\mathrm{O} 3$ & $4053.6(12)$ & $4452.4(12)$ & $4079.5(11)$ & $32.6(3)$ \\
\hline N1 & $6703.4(12)$ & $1684.9(10)$ & $2246.3(10)$ & $17.0(2)$ \\
\hline $\mathrm{N} 2$ & $8148.9(12)$ & $4262.0(11)$ & $995.7(10)$ & $16.9(2)$ \\
\hline $\mathrm{C} 4$ & $7171.1(13)$ & $3038.1(13)$ & $3128.8(12)$ & $17.7(3)$ \\
\hline $\mathrm{C} 1$ & $6781.6(13)$ & $3814.9(12)$ & $1916.2(12)$ & $16.5(3)$ \\
\hline $\mathrm{C} 12$ & $6966.6(14)$ & $2330.2(13)$ & $-435.4(12)$ & $18.2(3)$ \\
\hline $\mathrm{C} 10$ & $8252.4(13)$ & $3944.7(12)$ & $32.0(12)$ & $16.7(3)$ \\
\hline $\mathrm{C} 2$ & $6061.8(13)$ & $2846.4(12)$ & $1515.0(12)$ & $15.8(3)$ \\
\hline C3 & $7112.4(13)$ & $1811.7(13)$ & $3250.2(12)$ & $17.8(3)$ \\
\hline C18 & $4330.5(14)$ & $2747.9(12)$ & $1828.4(13)$ & $17.8(3)$ \\
\hline $\mathrm{C} 11$ & $6769.7(13)$ & $3277.3(13)$ & $136.8(12)$ & $17.0(3)$ \\
\hline $\mathrm{C} 23$ & $3618.5(15)$ & $2264.2(14)$ & $3053.5(13)$ & $22.1(3)$ \\
\hline $\mathrm{C} 8$ & $7464.3(15)$ & $881.6(14)$ & $4279.8(13)$ & $22.7(3)$ \\
\hline C19 & $3429.0(15)$ & $3064.0(14)$ & $946.4(13)$ & $22.1(3)$ \\
\hline $\mathrm{C} 25$ & $2684.1(16)$ & $4410.5(15)$ & $4381.4(14)$ & $25.3(3)$ \\
\hline C9 & $6047.5(15)$ & $504.0(13)$ & $2406.5(14)$ & $22.2(3)$ \\
\hline $\mathrm{C} 17$ & $6041.8(15)$ & $2315.9(14)$ & $-1236.6(14)$ & $22.7(3)$ \\
\hline $\mathrm{C} 13$ & $8083.9(15)$ & $1462.1(14)$ & $-199.6(14)$ & $22.9(3)$ \\
\hline $\mathrm{C} 5$ & $7611.4(15)$ & $3371.6(14)$ & $3999.5(13)$ & $23.0(3)$ \\
\hline $\mathrm{C} 16$ & $6194.3(17)$ & $1438.3(15)$ & $-1758.6(15)$ & $27.6(3)$ \\
\hline $\mathrm{C} 21$ & $1167.6(15)$ & $2391.2(14)$ & $2486.5(15)$ & $26.2(3)$ \\
\hline $\mathrm{C} 22$ & $2047.8(15)$ & $2098.0(15)$ & $3378.0(14)$ & $25.3(3)$ \\
\hline $\mathrm{C} 20$ & $1855.0(15)$ & $2876.7(15)$ & $1276.1(15)$ & $25.6(3)$ \\
\hline $\mathrm{C} 14$ & $8215.9(16)$ & $570.4(15)$ & $-705.4(15)$ & $26.6(3)$ \\
\hline $\mathrm{C} 7$ & $7911.8(16)$ & $1224.8(15)$ & $5157.5(14)$ & $26.7(3)$ \\
\hline $\mathrm{C} 15$ & $7263.0(17)$ & $547.2(15)$ & $-1478.5(15)$ & $27.5(3)$ \\
\hline C6 & $7991.3(17)$ & $2447.3(16)$ & $5029.3(14)$ & $27.9(3)$ \\
\hline $\mathrm{C} 26$ & $2956.3(17)$ & $3176.5(16)$ & $6397.3(15)$ & $29.6(3)$ \\
\hline $\mathrm{C} 24$ & $1609.6(19)$ & $5013.6(19)$ & $3550.4(17)$ & $33.1(4)$ \\
\hline $\mathrm{C} 27$ & $1944(2)$ & $2606.1(19)$ & $7624.1(17)$ & $41.1(4)$ \\
\hline
\end{tabular}


Table S3 Anisotropic Displacement Parameters $\left(\AA^{2} \times 10^{3}\right)$ for $6 \mathrm{~m}$. The Anisotropic displacement factor exponent takes the form: $-2 \pi^{2}\left[h^{2} a * 2 U_{11}+2 h k a * b * U_{12}+\ldots\right]$.

\begin{tabular}{|c|c|c|c|c|c|c|}
\hline Atom & $\mathbf{U}_{11}$ & $\mathbf{U}_{22}$ & $\mathbf{U}_{\mathbf{3 3}}$ & $\mathbf{U}_{\mathbf{2 3}}$ & $\mathbf{U}_{13}$ & $\mathbf{U}_{12}$ \\
\hline $\mathrm{O} 1$ & $14.3(4)$ & $20.8(5)$ & $23.4(5)$ & $-9.2(4)$ & $-1.6(4)$ & $3.1(4)$ \\
\hline $\mathrm{O} 2$ & $11.3(4)$ & $29.7(5)$ & $23.0(5)$ & $-12.7(4)$ & $-0.2(3)$ & $-3.3(4)$ \\
\hline $\mathrm{O} 4$ & $16.7(5)$ & $34.6(6)$ & $28.8(6)$ & $-11.1(5)$ & $-2.5(4)$ & $1.9(4)$ \\
\hline $\mathrm{O} 3$ & $17.1(5)$ & $51.1(8)$ & $32.4(6)$ & $-21.3(6)$ & $0.7(4)$ & $-1.2(5)$ \\
\hline N1 & $12.2(5)$ & $16.8(6)$ & $22.6(6)$ & $-7 \cdot 3(5)$ & $-5.1(4)$ & $0.8(4)$ \\
\hline $\mathrm{N} 2$ & $9.2(5)$ & $20.6(6)$ & $21.9(6)$ & $-9.4(5)$ & $-1.6(4)$ & $-2.9(4)$ \\
\hline $\mathrm{C} 4$ & $9.3(5)$ & $23.0(7)$ & $20.8(6)$ & $-8.7(5)$ & $-1.4(4)$ & $-0.5(5)$ \\
\hline $\mathrm{C} 1$ & $10.3(5)$ & $18.7(6)$ & $21.1(6)$ & $-8.7(5)$ & $-2.1(5)$ & $0.1(4)$ \\
\hline $\mathrm{C} 12$ & $11.2(5)$ & $22.5(7)$ & $21.5(6)$ & $-9.5(5)$ & $-1.9(5)$ & $-2.4(5)$ \\
\hline $\mathrm{C} 10$ & $9.9(5)$ & $18.6(6)$ & $22.0(6)$ & $-7.7(5)$ & $-4.1(5)$ & $0.4(5)$ \\
\hline $\mathrm{C} 2$ & $9.8(6)$ & $16.4(6)$ & $21.3(6)$ & $-6.8(5)$ & $-3.5(5)$ & $0.5(4)$ \\
\hline $\mathrm{C} 3$ & $8.0(5)$ & $22.9(7)$ & $21.8(7)$ & $-8.1(5)$ & $-1.4(4)$ & $-1.0(4)$ \\
\hline $\mathrm{C} 18$ & $9.5(6)$ & $18.5(6)$ & $26.3(7)$ & $-9.4(5)$ & $-3.4(5)$ & $0.3(4)$ \\
\hline $\mathrm{C} 11$ & $9.0(5)$ & $21.3(7)$ & $21.5(7)$ & $-8.5(5)$ & $-3.8(4)$ & $-0.5(5)$ \\
\hline $\mathrm{C} 23$ & $11.6(6)$ & $27.0(7)$ & $26.2(7)$ & $-8 \cdot 3(6)$ & $-3.7(5)$ & $0.3(5)$ \\
\hline $\mathrm{C} 8$ & $16.3(6)$ & $23.7(7)$ & $25.3(7)$ & $-5.8(6)$ & $-4.5(5)$ & $-0.7(5)$ \\
\hline C19 & $12.4(6)$ & $27.4(7)$ & $25.1(7)$ & $-7.7(6)$ & $-4.9(5)$ & $0.5(5)$ \\
\hline $\mathrm{C} 25$ & $20.7(7)$ & $30.5(8)$ & $27.6(7)$ & $-16.0(6)$ & $-0.6(5)$ & $-2.0(6)$ \\
\hline C9 & $17.6(6)$ & $20.4(7)$ & $29.6(7)$ & $-9.9(6)$ & $-5.6(5)$ & $-1.1(5)$ \\
\hline $\mathrm{C} 17$ & $15.5(6)$ & $27.1(7)$ & $28.6(7)$ & $-12.1(6)$ & $-8.2(5)$ & $-0.2(5)$ \\
\hline $\mathrm{C} 13$ & $14.1(6)$ & $31.7(8)$ & $28.0(7)$ & $-16.2(6)$ & $-6.3(5)$ & $2.4(5)$ \\
\hline $\mathrm{C} 5$ & $17.9(6)$ & $27.8(8)$ & $25.1(7)$ & $-11.9(6)$ & $-3.7(5)$ & $-1.6(5)$ \\
\hline $\mathrm{C} 16$ & $23.0(7)$ & $33.8(8)$ & $32.9(8)$ & $-17.9(7)$ & $-10.7(6)$ & $-1.1(6)$ \\
\hline $\mathrm{C} 21$ & $7.6(6)$ & $27.6(8)$ & $39.1(9)$ & $-8.0(6)$ & $-3.8(5)$ & $1.2(5)$ \\
\hline $\mathrm{C} 22$ & $12.0(6)$ & $29.4(8)$ & $29.5(8)$ & $-7.5(6)$ & $0.3(5)$ & $-0.2(5)$ \\
\hline $\mathrm{C} 20$ & $12.0(6)$ & $29.5(8)$ & $34.5(8)$ & $-9.4(6)$ & $-8 \cdot 3(5)$ & $1.5(5)$ \\
\hline $\mathrm{C} 14$ & $18.0(6)$ & $31.2(8)$ & $34.9(8)$ & $-17.6(7)$ & $-5.6(6)$ & $5.6(6)$ \\
\hline $\mathrm{C} 7$ & $22.3(7)$ & $32.1(8)$ & $21.8(7)$ & $-4.4(6)$ & $-7.1(5)$ & $0.2(6)$ \\
\hline $\mathrm{C} 15$ & $24.2(7)$ & $32.0(8)$ & $32.9(8)$ & $-19.9(7)$ & $-3.6(6)$ & $-2.0(6)$ \\
\hline C6 & $25.4(7)$ & $37.1(9)$ & $23.8(7)$ & $-12.9(7)$ & $-7.5(6)$ & $-1.6(6)$ \\
\hline $\mathrm{C} 26$ & $22.1(7)$ & $35.0(9)$ & $31.8(8)$ & $-12.6(7)$ & $-6.0(6)$ & $6.1(6)$ \\
\hline $\mathrm{C} 24$ & $25.3(8)$ & $41.9(10)$ & $32.0(9)$ & $-13.1(8)$ & $-7 \cdot 3(6)$ & $0.2(7)$ \\
\hline $\mathrm{C} 27$ & $35.1(9)$ & $47.8(11)$ & $33.8(9)$ & $-11.4(8)$ & $-1 \cdot 3(7)$ & 7.1 ( 8 ) \\
\hline
\end{tabular}

Table S4 Bond Lengths for 5p.

Atom Atom Length $/ \AA$ Atom Atom Length $/ \AA$

$\begin{array}{lclccc}\mathrm{O} 1 & \mathrm{C} 1 & 1.4071(15) & \mathrm{C} 2 & \mathrm{C} 18 & 1.5291(16) \\ \mathrm{O} 2 & \mathrm{C} 10 & 1.2316(16) & \mathrm{C} 2 & \mathrm{C} 11 & 1.5564(18) \\ \mathrm{O} 4 & \mathrm{C} 25 & 1.3345(19) & \mathrm{C} 3 & \mathrm{C} 8 & 1.393(2) \\ \mathrm{O} 4 & \mathrm{C} 26 & 1.4595(18) & \mathrm{C} 18 & \mathrm{C} 23 & 1.399(2) \\ \mathrm{O} 3 & \mathrm{C} 25 & 1.2142(18) & \mathrm{C} 18 & \mathrm{C} 19 & 1.3933(19) \\ \mathrm{N} 1 & \mathrm{C} 2 & 1.4844(16) & \mathrm{C} 23 & \mathrm{C} 22 & 1.3924(18)\end{array}$


Table S4 Bond Lengths for 5p.

\begin{tabular}{|c|c|c|c|c|c|}
\hline \multicolumn{2}{|c|}{ Atom Atom } & \multirow{2}{*}{$\begin{array}{c}\text { Length/A } \\
1.3937(17)\end{array}$} & \multicolumn{2}{|c|}{ Atom Atom } & Length/Å \\
\hline N1 & C3 & & $\mathrm{C} 8$ & $\mathrm{C} 7$ & $1.396(2)$ \\
\hline N1 & C9 & $1.4537(18)$ & C19 & $\mathrm{C} 20$ & $1.3974(18)$ \\
\hline $\mathrm{N} 2$ & $\mathrm{C} 1$ & $1.4525(16)$ & $\mathrm{C} 25$ & $\mathrm{C} 24$ & $1.493(2)$ \\
\hline N2 & $\mathrm{C} 10$ & $1.3391(18)$ & $\mathrm{C} 17$ & $\mathrm{C} 16$ & $1.390(2)$ \\
\hline $\mathrm{C} 4$ & $\mathrm{C} 1$ & $1.5053(18)$ & $\mathrm{C} 13$ & $\mathrm{C} 14$ & 1.3 \\
\hline $\mathrm{C} 4$ & $\mathrm{C} 3$ & $1.393(2)$ & $\mathrm{C} 5$ & C6 & $1.400(2)$ \\
\hline $\mathrm{C} 4$ & $\mathrm{C} 5$ & $1.3784(19)$ & $\mathrm{C} 16$ & $\mathrm{C} 15$ & $1.387(2)$ \\
\hline $\mathrm{C} 1$ & $\mathrm{C} 2$ & $1.5912(18)$ & $\mathrm{C} 21$ & $\mathrm{C} 22$ & $1.390(2)$ \\
\hline $\mathrm{C} 12$ & $\mathrm{C} 11$ & $1.5074(18)$ & $\mathrm{C} 21$ & $\mathrm{C} 20$ & $1.378(2)$ \\
\hline $\mathrm{C} 12$ & $\mathrm{C} 17$ & $1.3963(18)$ & C14 & $\mathrm{C} 15$ & $1.391(2)$ \\
\hline $\mathrm{C} 12$ & C13 & $1.3974(19)$ & $\mathrm{C} 7$ & C6 & $1.387(2)$ \\
\hline $\mathrm{C} 10$ & C11 & $1.5286(16)$ & $\mathrm{C} 26$ & $\mathrm{C} 27$ & $1.502(2)$ \\
\hline
\end{tabular}

Table S5 Bond Angles for 5p.

\begin{tabular}{|c|c|c|c|c|c|c|c|}
\hline \multicolumn{3}{|c|}{ Atom Atom Atom } & \multirow{2}{*}{$\begin{array}{c}\text { Angle } /^{\circ} \\
116.21(11)\end{array}$} & \multicolumn{3}{|c|}{ Atom Atom Atom } & \multirow{2}{*}{$\begin{array}{c}\text { Angle }^{\circ} \\
120.40(13)\end{array}$} \\
\hline $\mathrm{C} 25$ & $\mathrm{O} 4$ & $\mathrm{C} 26$ & & $\mathrm{C} 4$ & $\mathrm{C} 3$ & $\mathrm{C} 8$ & \\
\hline C3 & N1 & $\mathrm{C} 2$ & $109.38(11)$ & $\mathrm{C} 8$ & $\mathrm{C} 3$ & N1 & $27.52(13)$ \\
\hline C3 & N1 & C9 & $8.17(11)$ & $\mathrm{C} 23$ & $\mathrm{C} 18$ & $\mathrm{C} 2$ & $118.72(12)$ \\
\hline C9 & N1 & $\mathrm{C} 2$ & $20.01(10)$ & $\mathrm{C} 19$ & $\mathrm{C} 18$ & $\mathrm{C} 2$ & $122.85(12)$ \\
\hline $\mathrm{C} 10$ & $\mathrm{~N} 2$ & $\mathrm{C} 1$ & $15.58(11)$ & C19 & $\mathrm{C} 18$ & $\mathrm{C} 23$ & $118.35(12)$ \\
\hline $\mathrm{C} 3$ & $\mathrm{C} 4$ & $\mathrm{C} 1$ & $7.75(11)$ & $\mathrm{C} 12$ & $\mathrm{C} 11$ & C10 & $13.56(10)$ \\
\hline C5 & $\mathrm{C} 4$ & $\mathrm{C} 1$ & 0.69 ( & $\mathrm{C} 12$ & $\mathrm{C} 11$ & $\mathrm{C}^{2}$ & $18.41(11)$ \\
\hline C5 & $\mathrm{C} 4$ & $\mathrm{C} 3$ & $.45(13)$ & $\mathrm{C} 10$ & $\mathrm{C} 11$ & $\mathrm{C} 2$ & $103.12(10)$ \\
\hline O1 & $\mathrm{C} 1$ & N2 & \pm( & $\mathrm{C} 22$ & $\mathrm{C} 23$ & $\mathrm{C} 18$ & 13) \\
\hline O1 & $\mathrm{C} 1$ & $\mathrm{C} 4$ & $93(11)$ & C3 & $\mathrm{C} 8$ & C7 & $14)$ \\
\hline O1 & $\mathrm{C} 1$ & $\mathrm{C} 2$ & $15.35(10)$ & C18 & C19 & $\mathrm{C} 20$ & $120.87(13)$ \\
\hline N2 & $\mathrm{C} 1$ & $\mathrm{C} 4$ & $10.55(10)$ & O4 & $\mathrm{C} 25$ & $\mathrm{C} 24$ & $12.84(13)$ \\
\hline N2 & $\mathrm{C} 1$ & $\mathrm{C} 2$ & $2.52(10)$ & O3 & $\mathrm{C} 25$ & $\mathrm{O} 4$ & (15) \\
\hline $\mathrm{C} 4$ & $\mathrm{C} 1$ & $\mathrm{C} 2$ & 811 & $\mathrm{O} 3$ & $\mathrm{C} 25$ & $\mathrm{C} 24$ & 1 \\
\hline C17 & $\mathrm{C} 12$ & C11 & ) & C16 & C17 & $\mathrm{C} 12$ & \\
\hline $\mathrm{C} 17$ & $\mathrm{C} 12$ & $\mathrm{C} 13$ & 118 & C14 & $\mathrm{C} 13$ & $\mathrm{C} 12$ & 120.5 \\
\hline $\mathrm{C} 13$ & $\mathrm{C} 12$ & $\mathrm{C} 11$ & 41( & 4 & $\mathrm{C} 5$ & C6 & $118.69(14)$ \\
\hline $\mathrm{O} 2$ & $\mathrm{C} 10$ & N2 & 02( & C15 & $\mathrm{C} 16$ & $\mathrm{C} 1$ & (14) \\
\hline $\mathrm{O} 2$ & $\mathrm{C} 10$ & $\mathrm{C} 11$ & $21(12)$ & $\mathrm{C} 20$ & $\mathrm{C} 21$ & $\mathrm{C} 22$ & $19.64(12)$ \\
\hline N2 & $\mathrm{C} 10$ & $\mathrm{C} 11$ & $108.74(11)$ & $\mathrm{C} 21$ & $\mathrm{C} 22$ & $\mathrm{C} 23$ & $120.33(14)$ \\
\hline N1 & $\mathrm{C} 2$ & $\mathrm{C} 1$ & $101.31(10)$ & $\mathrm{C} 21$ & $\mathrm{C} 20$ & C19 & $120.21(13)$ \\
\hline N1 & $\mathrm{C} 2$ & $\mathrm{C} 18$ & $110.47(10)$ & C15 & $\mathrm{C} 14$ & $\mathrm{C} 13$ & $120.55(14)$ \\
\hline N1 & $\mathrm{C} 2$ & C11 & $111.84(10)$ & C6 & C7 & $\mathrm{C} 8$ & $121.75(14)$ \\
\hline $\mathrm{C} 18$ & $\mathrm{C} 2$ & $\mathrm{C} 1$ & $113.46(10)$ & $\mathrm{C} 16$ & C15 & C14 & $119.23(14)$ \\
\hline $\mathrm{C} 18$ & $\mathrm{C} 2$ & C11 & $15.61(11)$ & C7 & C6 & $\mathrm{C} 5$ & $119.80(14)$ \\
\hline C11 & $\mathrm{C} 2$ & $\mathrm{C} 1$ & $03.06(10)$ & O4 & $\mathrm{C} 26$ & $\mathrm{C} 27$ & 107 \\
\hline C. & & 1 & $112.07(12)$ & & & & \\
\hline
\end{tabular}


Table S6 Torsion Angles for 5p.

\begin{tabular}{|c|c|c|c|c|}
\hline $\mathbf{A}$ & D & Angle/ ${ }^{\circ}$ & D & Angle ${ }^{\circ}$ \\
\hline $\mathrm{O} 1$ & C1 $\mathrm{C} 2 \quad \mathrm{~N} 1$ & $-150.10(11)$ & C3 N1 C2 C11 & $131.97(11)$ \\
\hline $\mathrm{O} 1$ & $\mathrm{C} 1 \mathrm{C} 2 \mathrm{C} 18$ & $-31.71(16)$ & $\begin{array}{llll}\mathrm{C} 3 & \mathrm{C} 4 & \mathrm{C} 1 & \mathrm{O} 1\end{array}$ & $142.35(11)$ \\
\hline 1 & $\mathrm{C} 1 \mathrm{C} 2 \mathrm{C} 11$ & $94.05(12)$ & C3 $\quad \mathrm{C} 4 \quad \mathrm{C} 1 \quad \mathrm{~N} 2$ & $-94.54(12)$ \\
\hline $\mathrm{O} 2$ & C10 C11 C12 & $32.42(19)$ & $\mathrm{C} 3 \mathrm{C}_{4} \mathrm{C} 1 \mathrm{C} 2$ & $14.77(13)$ \\
\hline $\mathrm{O} 2$ & $\mathrm{C} 10 \mathrm{C} 11 \mathrm{C} 2$ & $161.81(13)$ & C3 $\mathrm{C} 4 \quad \mathrm{C} 5 \quad \mathrm{C} 6$ & $-0.6(2)$ \\
\hline N1 & C2 C18 C23 & $46.22(16)$ & C3 $\quad$ C8 $\quad$ C7 $\quad$ C6 & $0.8(2)$ \\
\hline N1 & C2 C18C19 & $-130.61(14)$ & $\mathrm{C} 18 \mathrm{C} 2 \mathrm{C} 11 \mathrm{C} 12$ & $-83.55(14)$ \\
\hline N1 & C2 C11 C12 & $44.03(14)$ & $\mathrm{C} 18 \mathrm{C} 2 \mathrm{C} 1$ & $150.11(11)$ \\
\hline$\sqrt{1}$ & $\mathrm{C} 2 \mathrm{C} 11 \mathrm{C} 10$ & $-82.31(12)$ & $\mathrm{C} 18 \mathrm{C} 23 \mathrm{C} 22 \mathrm{C} 21$ & $1.1(2)$ \\
\hline N1 & C3 $\mathrm{C} 8 \mathrm{C} 7$ & $177.20(12)$ & C18C19C20C21 & $1.0(2)$ \\
\hline N2 & C1 1 C2 N1 & $92.99(11)$ & $\mathrm{C} 11 \mathrm{C} 12 \mathrm{C} 17 \mathrm{C} 16$ & $-179.09(13)$ \\
\hline $\mathrm{N} 2$ & $\mathrm{C} 1 \mathrm{C} 2 \mathrm{C} 18$ & $-148.62(11)$ & C11C12C13C14 & $177.91(13)$ \\
\hline N2 & $\mathrm{C} 1 \mathrm{C} 2 \mathrm{C} 11$ & $-22.86(12)$ & $\mathrm{C} 11 \mathrm{C} 2 \mathrm{C} 18 \mathrm{C} 23$ & $174.49(12)$ \\
\hline N2 & C10C11 C12 & $-149.62(12)$ & C11 C2 C18C19 & $-2.34(19)$ \\
\hline N2 & $\mathrm{C} 10 \mathrm{C} 11 \mathrm{C} 2$ & $-20.23(14)$ & C $23 \mathrm{C} 18 \mathrm{C} 19 \mathrm{C} 20$ & $-1.4(2)$ \\
\hline $\mathrm{C4}$ & $\mathrm{C} 1 \mathrm{C} 2 \mathrm{~N} 1$ & $-22.16(12)$ & C8 C7 C6 C5 & $0.2(2)$ \\
\hline $\mathrm{C} 4$ & $\mathrm{C} 1 \mathrm{C} 2 \mathrm{C} 18$ & $96.23(12)$ & C19C18C23C22 & $0.4(2)$ \\
\hline $\mathrm{C} 4$ & $\mathrm{C} 1 \mathrm{C} 2 \mathrm{C} 11$ & $-138.01(10)$ & $\mathrm{C} 25 \mathrm{O} 4 \mathrm{C} 26 \mathrm{C} 27$ & $177.87(14)$ \\
\hline $\mathrm{C} 4$ & C3 $\mathrm{C} 8 \mathrm{C} 7$ & $-1.69(19)$ & C9 $\mathrm{N} 1 \mathrm{C} 2 \mathrm{C} 1$ & $164.16(11)$ \\
\hline $\mathrm{C} 4$ & C5 C6 C7 & $-0.3(2)$ & C9 N1 C2 C18 & $43.63(16)$ \\
\hline $\mathrm{C} 1$ & $\mathrm{~N} 2 \mathrm{C} 10 \mathrm{O} 2$ & $-176.67(13)$ & C9 N1 C2 C11 & $-86.67(14)$ \\
\hline $\mathrm{C} 1$ & N2 C10C11 & $5.39(16)$ & C9 N1 C3 C4 & $-157.24(11)$ \\
\hline $\mathrm{C} 1$ & C4 C3 N1 & $-0.76(14)$ & C9 N1 C3 C8 & $23.79(19)$ \\
\hline $\mathrm{C} 1$ & $\mathrm{C} 4 \mathrm{C} 3 \mathrm{C} 8$ & $178.29(11)$ & $\mathrm{C} 17 \mathrm{C} 12 \mathrm{C} 11 \mathrm{C} 10$ & $-130.73(13$ \\
\hline $\mathrm{C} 1$ & $\mathrm{C} 4 \mathrm{C} 5 \mathrm{C} 6$ & $-176.40(13)$ & $\mathrm{C} 17 \mathrm{C} 12 \mathrm{C} 11 \mathrm{C} 2$ & $108.11(14$ \\
\hline $\mathrm{C} 1$ & $\mathrm{C} 2 \mathrm{C} 18 \mathrm{C} 23$ & $-66.74(16)$ & $\mathrm{C} 13 \mathrm{C} 14$ & $-3.1(2)$ \\
\hline $\mathrm{C} 1$ & C2 C18C19 & $116.43(14)$ & $\mathrm{C} 17 \mathrm{C} 16 \mathrm{C} 15 \mathrm{C} 14$ & $-2.4(2)$ \\
\hline $\mathrm{C} 1$ & C2 C11 C12 & $152.09(10)$ & $\mathrm{C} 13 \mathrm{C} 12 \mathrm{C} 11 \mathrm{C} 10$ & $48.18(17)$ \\
\hline $\mathrm{C} 1$ & $\mathrm{C} 2 \mathrm{C} 11 \mathrm{C} 10$ & $25.74(12)$ & $\mathrm{C} 13 \mathrm{C} 12 \mathrm{C} 11 \mathrm{C} 2$ & $-72.97(16$ \\
\hline 12 & $\mathrm{C} 17 \mathrm{C} 16 \mathrm{C} 15$ & $0.8(2)$ & $\mathrm{C} 13 \mathrm{C} 12 \mathrm{C} 17 \mathrm{C} 16$ & $2.0(2)$ \\
\hline $\mathrm{C} 12$ & $\mathrm{C} 13 \mathrm{C} 14 \mathrm{C} 15$ & $1.6(2)$ & $\mathrm{C} 13 \mathrm{C} 14 \mathrm{C} 15 \mathrm{C} 16$ & $1.2(2)$ \\
\hline $\mathrm{C} 10$ & $\mathrm{~N} 2 \quad \mathrm{C} 1 \quad \mathrm{O} 1$ & $-110.60(13)$ & $\begin{array}{llll}\mathrm{C} 5 & \mathrm{C} 4 & \mathrm{C} 1 & \mathrm{O} 1\end{array}$ & $-41.42(19$ \\
\hline $\mathrm{C} 10$ & $\mathrm{~N} 2 \mathrm{C} 1 \mathrm{C} 4$ & $121.71(13)$ & $\begin{array}{llll}\mathrm{C} 5 & \mathrm{C} 4 & \mathrm{C} 1 & \mathrm{~N} 2\end{array}$ & $81.70(17)$ \\
\hline $\mathrm{C} 10$ & $\mathrm{~N} 2 \quad \mathrm{C} 1 \quad \mathrm{C} 2$ & $11.56(14)$ & $\begin{array}{llll}\mathrm{C} 5 & \mathrm{C} 4 & \mathrm{C} 1 & \mathrm{C} 2\end{array}$ & $-169.00(13$ \\
\hline $\mathrm{C} 2$ & N1 C3 C4 & $-15.07(14)$ & $\begin{array}{llll}\mathrm{C} 5 & \mathrm{C} 4 & \mathrm{C} 3 & \mathrm{~N} 1\end{array}$ & -177.41 ( 11 \\
\hline $\mathrm{C} 2$ & N1 C3 C8 & $165.96(12)$ & $\begin{array}{llll}\mathrm{C} 5 & \mathrm{C} 4 & \mathrm{C} 3 & \mathrm{C} 8\end{array}$ & $1.64(19)$ \\
\hline $\mathrm{C} 2$ & C18 C23 C22 & $-176.60(13)$ & $\mathrm{C} 22 \mathrm{C} 21 \mathrm{C} 20 \mathrm{C} 19$ & $0.5(2)$ \\
\hline $\mathrm{C} 2$ & C18C19C20 & $175.40(13)$ & $\mathrm{C} 20 \mathrm{C} 21 \mathrm{C} 22 \mathrm{C} 23$ & $-1.6(2)$ \\
\hline $\mathrm{C} 3$ & $\mathrm{~N} 1 \mathrm{C} 2 \mathrm{C} 1$ & $22.80(12)$ & $\mathrm{C} 26 \mathrm{O} 4 \mathrm{C} 25 \mathrm{O} 3$ & $-1.1(2)$ \\
\hline $\mathrm{C} 3$ & N1 C2 C18 & $-97.74(12)$ & $\mathrm{C} 26 \mathrm{O} 4 \mathrm{C} 25 \mathrm{C} 24$ & $179.28(14)$ \\
\hline
\end{tabular}


Table S7 Hydrogen Atom Coordinates $\left(\AA \times 10^{4}\right)$ and Isotropic Displacement Parameters $\left(\AA^{2} \times 10^{3}\right)$ for $5 p$.

\begin{tabular}{|c|c|c|c|c|}
\hline Atom & $x$ & $y$ & $z$ & $\mathbf{U}(\mathbf{e q})$ \\
\hline H11 & 6117.03 & 3901.24 & -304.15 & 20 \\
\hline $\mathrm{H} 23$ & 4199.16 & 2051.76 & 3656.45 & 27 \\
\hline H8 & 7403.07 & 58.13 & 4379.36 & 27 \\
\hline H19 & 3880.83 & 3404.14 & 127.42 & 27 \\
\hline H9A & 5279.88 & 221.79 & 3131.35 & 33 \\
\hline H9B & 6825.01 & -85.02 & 2477.07 & 33 \\
\hline H9C & 5604.62 & 595.24 & 1716.73 & 33 \\
\hline H17 & 5312.67 & 2902.57 & -1423.75 & 27 \\
\hline H13 & 8744.61 & 1480.75 & 299.58 & 27 \\
\hline H5 & 7655.26 & 4194.62 & 3904.46 & 28 \\
\hline H16 & 5577.21 & 1448.61 & -2298.2 & 33 \\
\hline $\mathrm{H} 21$ & 120.74 & 2260.42 & 2705.53 & 31 \\
\hline $\mathrm{H} 22$ & 1585.26 & 1789.3 & 4195.3 & 30 \\
\hline $\mathrm{H} 20$ & 1269.3 & 3080.35 & 676.98 & 31 \\
\hline H14 & 8947.5 & -15.07 & -525.09 & 32 \\
\hline $\mathrm{H} 7$ & 8163.25 & 617.47 & 5846.53 & 32 \\
\hline H15 & 7341.34 & -58.83 & -1803.88 & 33 \\
\hline H6 & 8296.1 & 2652.35 & 5625.94 & 33 \\
\hline $\mathrm{H} 26 \mathrm{~A}$ & 3687.8 & 3758.83 & 6409.84 & 36 \\
\hline $\mathrm{H} 26 \mathrm{~B}$ & 3501.04 & 2545.88 & 6173.19 & 36 \\
\hline $\mathrm{H} 27 \mathrm{~A}$ & 1210.57 & 2047.77 & 7593.92 & 62 \\
\hline $\mathrm{H} 27 \mathrm{~B}$ & 1432.66 & 3241.25 & 7844.31 & 62 \\
\hline $\mathrm{H} 27 \mathrm{C}$ & 2543.34 & 2164.5 & 8218.27 & 62 \\
\hline $\mathrm{H} 2$ & $8820(20)$ & $4740(20)$ & $1043(18)$ & $36(5)$ \\
\hline $\mathrm{H} 1$ & $5270(30)$ & $4650(20)$ & $2600(20)$ & 41 ( 6 ) \\
\hline $\mathrm{H} 24 \mathrm{~A}$ & $1820(40)$ & $4730(40)$ & $2910(40)$ & $24(13)$ \\
\hline H24B & $1880(50)$ & $5900(50)$ & $3180(40)$ & $41(16)$ \\
\hline $\mathrm{H} 24 \mathrm{C}$ & $570(60)$ & $4770(60)$ & $3970(50)$ & $58(17)$ \\
\hline H24D & $940(70)$ & $4390(60)$ & $3550(50)$ & $40(20)$ \\
\hline H24E & $2150(70)$ & $5450(70)$ & $2690(70)$ & $60(20)$ \\
\hline $\mathrm{H} 24 \mathrm{~F}$ & $810(60)$ & $5450(50)$ & $3900(50)$ & $32(17)$ \\
\hline
\end{tabular}

Table S8 Atomic Occupancy for 5p.

$\begin{array}{cccccc}\text { Atom } & \text { Occupancy } & \text { Atom } & \text { Occupancy } & \text { Atom } & \text { Occupancy } \\ \text { H24A } & 0.55(7) & \text { H24B } & 0.55(7) & \text { H24C } & 0.55(7) \\ \text { H24D } & 0.45(7) & \text { H24E } & 0.45(7) & \text { H24F } & 0.45(7)\end{array}$

\section{Experimental}

Single crystals of $\mathrm{C}_{27} \mathrm{H}_{28} \mathrm{~N}_{2} \mathrm{O}_{4} \mathbf{5 p}$ were prepared by slow evaporation of saturated solution in EtOAc. A suitable crystal was selected and mounted on the glass stick by acrylic glue on a SuperNova, Dual, $\mathrm{Cu}$ at home/near, AtlasS2 diffractometer. The crystal was kept at 125(30) K during data collection. Using Olex2 [1], the structure was solved with the SHELXT [2] structure solution program using Intrinsic Phasing and refined with the SHELXL [3] refinement package using Least Squares minimization. 


\section{Crystal structure determination of 5p}

Crystal Data for $\mathrm{C}_{27} \mathrm{H}_{28} \mathrm{~N}_{2} \mathrm{O}_{4}(M=444.51 \mathrm{~g} / \mathrm{mol})$ : triclinic, space group P-1 (no. 2), $a=8.9958(3) \AA, b=$ 11.7405(6) $\AA, c=12.0993(5) \AA, \alpha=67.721(4)^{\circ}, \beta=79.125(3)^{\circ}, \gamma=88.176(3)^{\circ}, V=1160.13(9) \AA^{3}, Z=2, T=$ $125(30) \mathrm{K}, \mu(\mathrm{Cu} \mathrm{K} \alpha)=0.691 \mathrm{~mm}^{-1}$, Dcalc $=1.272 \mathrm{~g} / \mathrm{cm}^{3}, 23047$ reflections measured $\left(8.044^{\circ} \leq 2 \Theta \leq 161.988^{\circ}\right)$, 4868 unique $\left(R_{\text {int }}=0.0599, R_{\text {sigma }}=0.0334\right)$ which were used in all calculations. The final $R_{1}$ was $0.0469(\mathrm{I}>2 \sigma(\mathrm{I}))$ and $w R_{2}$ was 0.1314 (all data).

\section{Refinement model description}

Details:

Number of restraints - 0, number of constraints - unknown.

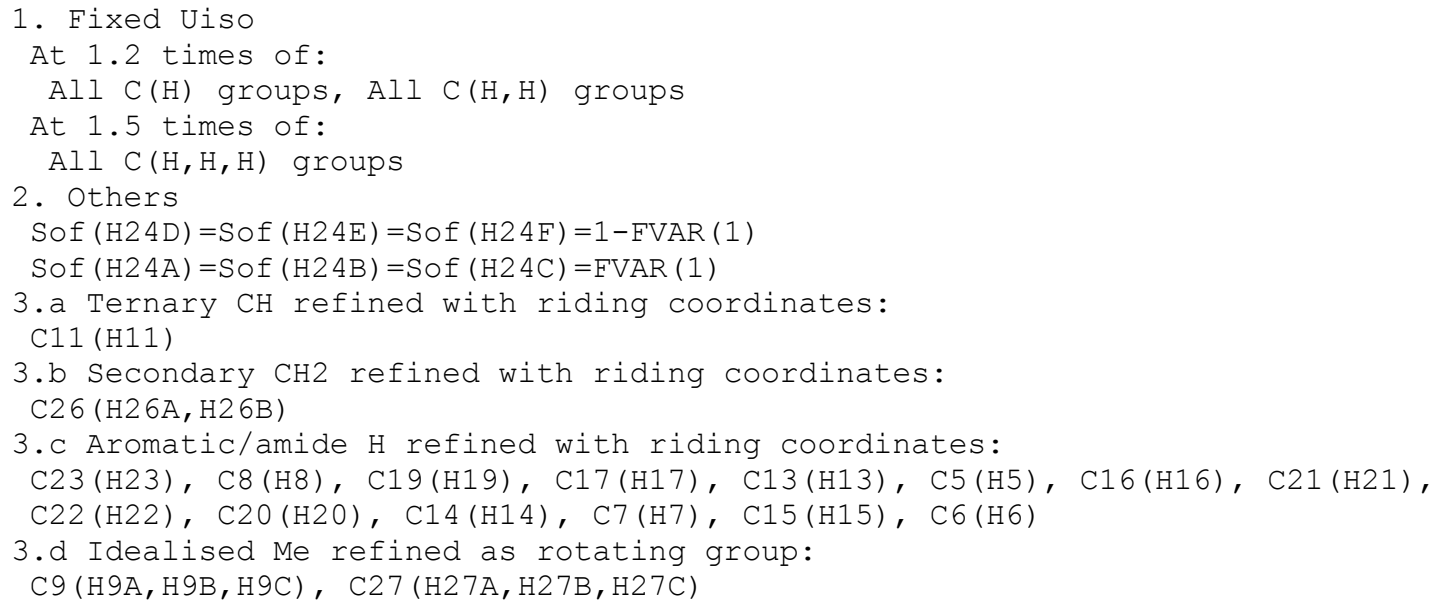

This report has been created with Olex2, compiled on 2020.11 .12 svn.r5f609507 for OlexSys. Please let us know if there are any errors or if you would like to have additional features. 


\section{X-Ray crystallography data for $6 a$}

\section{C \\ $\mathrm{H}$ \\ $Q^{N}$ \\ O}
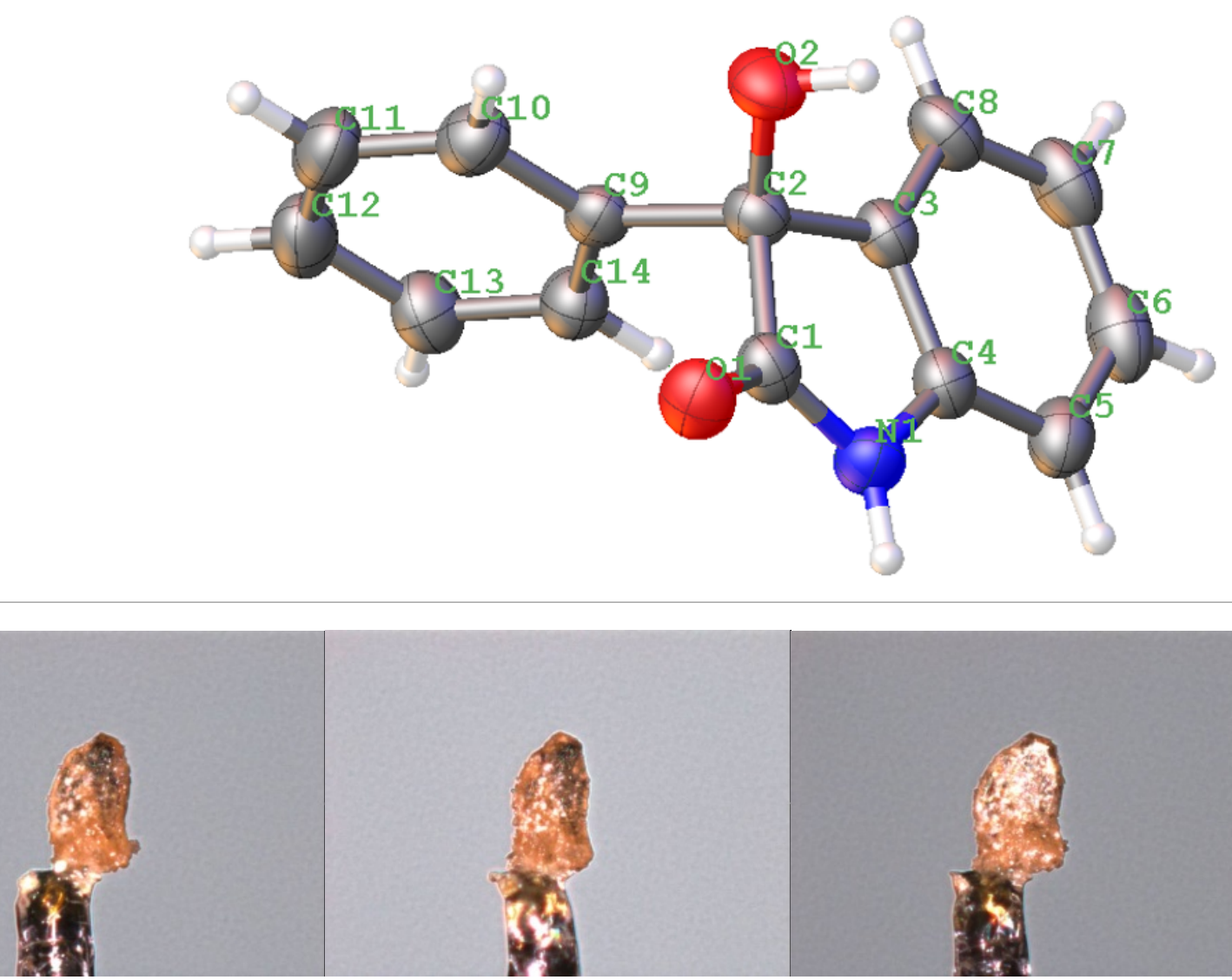

Figure S2. ORTEP drawing of the crystal structure showing 50\% probability thermal ellipsoids (top, CCDC 2105382) and microphotography of the single crystal of compound $\mathbf{6 a}$ used for XRay diffraction analysis at the bottom. 
Table S9 Crystal data and structure refinement for $6 \mathrm{a}$.

Identification code

ANNA VS12

Empirical formula

$\mathrm{C}_{14} \mathrm{H}_{11} \mathrm{NO}_{2}$

Formula weight

225.24

Temperature/K

$302.56(10)$

Crystal system

Space group

orthorhombic

$\mathrm{a} / \AA$

Pbca

$\mathrm{b} / \AA$

$10.3873(3)$

$\mathrm{c} / \AA$

$7.7716(3)$

$\alpha /{ }^{\circ}$

26.5900(10)

$\beta /{ }^{\circ}$

90

$\gamma /{ }^{\circ}$

90

Volume $/ \AA^{3}$

90

Z

2146.51(12)

8

$\rho_{\text {calc g }} / \mathrm{cm}^{3}$

1.394

$\mu / \mathrm{mm}^{-1}$

0.763

$\mathrm{F}(000)$

944.0

Crystal size $/ \mathrm{mm}^{3}$

$0.375 \times 0.229 \times 0.111$

Radiation

$\mathrm{Cu} \mathrm{K \alpha}(\lambda=1.54184)$

$2 \Theta$ range for data collection $/{ }^{\circ} 10.808$ to 152.706

Index ranges

$-9 \leq \mathrm{h} \leq 13,-9 \leq \mathrm{k} \leq 9,-33 \leq 1 \leq 32$

Reflections collected

11366

Independent reflections

$2223\left[\mathrm{R}_{\text {int }}=0.0805, \mathrm{R}_{\text {sigma }}=0.0408\right]$

Data/restraints/parameters

$2223 / 0 / 150$

Goodness-of-fit on $\mathrm{F}^{2}$

1.042

Final $R$ indexes $[\mathrm{I}>=2 \sigma(\mathrm{I})] \quad \mathrm{R}_{1}=0.0499, \mathrm{wR}_{2}=0.1377$

Final $\mathrm{R}$ indexes [all data] $\quad \mathrm{R}_{1}=0.0567, \mathrm{wR}_{2}=0.1485$

Largest diff. peak/hole / e $\AA^{-3} 0.24 /-0.26$ 
Table S10 Fractional Atomic Coordinates $\left(\times 10^{4}\right)$ and Equivalent Isotropic Displacement Parameters $\left(\AA^{2} \times 10^{3}\right)$ for $6 a$. $U_{\text {eq }}$ is defined as $1 / 3$ of of the trace of the orthogonalised UIJ tensor.

$\begin{array}{cc}\text { Atom } & \boldsymbol{x} \\ \mathrm{O} 1 & 3791.8(11) \\ \mathrm{O} 2 & 2807.1(10) \\ \mathrm{N} 1 & 5186.5(13) \\ \mathrm{C} 9 & 4780.1(8) \\ \mathrm{C} 14 & 6109.6(8) \\ \mathrm{C} 13 & 6719.2(7) \\ \mathrm{C} 12 & 5999.2(10) \\ \mathrm{C} 11 & 4669.7(10) \\ \mathrm{C} 10 & 4060.1(7) \\ \mathrm{C} 3 & 4834.7(14) \\ \mathrm{C} 2 & 4123.2(13) \\ \mathrm{C} 1 & 4322.9(14) \\ \mathrm{C} 4 & 5481.6(14) \\ \mathrm{C} 8 & 4937.2(16) \\ \mathrm{C} 5 & 6264.3(15) \\ \mathrm{C} 6 & 6352.8(16) \\ \mathrm{C} 7 & 5699.0(18)\end{array}$

$\boldsymbol{y}$
$10324.6(15)$
$7668.4(16)$
$8122.3(18)$
$8871.2(12)$
$8790.2(13)$
$9781.2(15)$
$10853.1(15)$
$10934.1(14)$
$9943.1(14)$
$6232.6(18)$
$7866.5(18)$
$8949.5(19)$
$6480.6(19)$
$4694(2)$
$5230(2)$
$3680(2)$
$3406(2)$

$\boldsymbol{z}$
$5556.4(4)$
$6257.4(5)$
$5362.3(5)$
$6566.1(3)$
$6620.5(4)$
$6984.7(4)$
$7294.6(4)$
$7240.2(4)$
$6875.9(4)$
$6008.1(6)$
$6140.6(6)$
$5653.4(6)$
$5557.3(6)$
$6263.1(7)$
$5348.5(7)$
$5611.6(8)$
$6055.2(8)$

$\mathrm{U}(\mathbf{e q})$

$47.2(3)$

$49.0(3)$

$42.5(3)$

$35.6(3)$

$42.6(4)$

$50.1(4)$

$54.1(4)$

$55.1(5)$

$45.7(4)$

$37.3(3)$

$35.8(3)$

$37.4(3)$

$38.6(4)$

$46.2(4)$

$47.4(4)$

$53.7(5)$

$53.2(5)$

Table S11 Anisotropic Displacement Parameters $\left(\AA^{2} \times 10^{3}\right)$ for 6a. The Anisotropic displacement factor exponent takes the form: $-2 \pi^{2}\left[h^{2} a * 2 U_{11}+2 h k a * b * U_{12}+\ldots\right]$.

$\begin{array}{ccccccc}\text { Atom } & \mathbf{U}_{11} & \mathbf{U}_{22} & \mathbf{U}_{33} & \mathbf{U}_{23} & \mathbf{U}_{13} & \mathbf{U}_{12} \\ \text { O1 } & 58.3(7) & 37.4(6) & 45.8(6) & 6.3(5) & -3.6(5) & 7.5(5) \\ \text { O2 } & 38.2(6) & 48.4(7) & 60.3(8) & -2.5(5) & 6.2(5) & -4.9(5) \\ \text { N1 } & 52.4(7) & 37.0(7) & 38.1(7) & 3.8(5) & 4.2(5) & -0.4(5) \\ \text { C9 } & 43.7(7) & 28.9(7) & 34.0(7) & 4.6(5) & 2.4(6) & 0.0(5) \\ \text { C14 } & 44.7(7) & 39.4(8) & 43.8(8) & -3.7(6) & 1.2(6) & 1.0(6) \\ \text { C13 } & 51.4(8) & 45.4(9) & 53.3(10) & -2.4(7) & -7.1(7) & -3.8(7) \\ \text { C12 } & 74.2(11) & 45.0(9) & 43.1(9) & -6.6(7) & -5.0(8) & -7.2(8) \\ \text { C11 } & 73.6(11) & 45.8(9) & 45.9(9) & -9.2(7) & 9.1(8) & 4.0(8) \\ \text { C10 } & 49.5(8) & 42.5(8) & 45.2(9) & -0.5(6) & 6.3(6) & 5.8(7) \\ \text { C3 } & 39.3(7) & 29.3(7) & 43.5(8) & -0.8(6) & -2.7(6) & -2.2(5) \\ \text { C2 } & 37.0(7) & 31.2(7) & 39.2(7) & 2.8(5) & 1.2(5) & -0.6(5) \\ \text { C1 } & 42.5(7) & 32.2(7) & 37.4(7) & 0.6(5) & -5.0(6) & -1.8(6) \\ \text { C4 } & 40.4(7) & 33.2(7) & 42.0(8) & -2.8(6) & -3.1(6) & -2.3(6) \\ \text { C8 } & 49.9(8) & 33.4(8) & 55.4(10) & 6.6(6) & -4.7(7) & -4.9(6) \\ \text { C5 } & 44.4(8) & 46.2(9) & 51.7(9) & -12.1(7) & 0.8(7) & 1.0(6) \\ \text { C6 } & 47.1(8) & 38.8(9) & 75.2(13) & -15.5(8) & -12.8(8) & 7.4(7) \\ \text { C7 } & 56.6(9) & 29.8(8) & 73.2(12) & 1.2(7) & -13.1(9) & 1.2(7)\end{array}$


Table S12 Bond Lengths for $6 a$.

\begin{tabular}{|c|c|c|c|c|c|}
\hline \multicolumn{2}{|c|}{ Atom Atom } & Length/Å & Atom & Atom & Length/Å \\
\hline $\mathrm{O} 1$ & $\mathrm{C} 1$ & $1.2300(18)$ & $\mathrm{C} 11$ & $\mathrm{C} 10$ & 1.3900 \\
\hline $\mathrm{O} 2$ & $\mathrm{C} 2$ & $1.4103(17)$ & $\mathrm{C} 3$ & $\mathrm{C} 2$ & $1.5108(19)$ \\
\hline N1 & $\mathrm{C} 1$ & $1.348(2)$ & $\mathrm{C} 3$ & $\mathrm{C} 4$ & $1.388(2)$ \\
\hline N1 & $\mathrm{C} 4$ & $1.4109(19)$ & $\mathrm{C} 3$ & $\mathrm{C} 8$ & $1.379(2)$ \\
\hline C9 & $\mathrm{C} 14$ & 1.3900 & $\mathrm{C} 2$ & $\mathrm{C} 1$ & $1.559(2)$ \\
\hline $\mathrm{C} 9$ & $\mathrm{C} 10$ & 1.3900 & $\mathrm{C} 4$ & $\mathrm{C} 5$ & $1.384(2)$ \\
\hline C9 & $\mathrm{C} 2$ & $1.5346(16)$ & $\mathrm{C} 8$ & $\mathrm{C} 7$ & $1.390(3)$ \\
\hline $\mathrm{C} 14$ & C13 & 1.3900 & $\mathrm{C} 5$ & C6 & $1.396(3)$ \\
\hline $\mathrm{C} 13$ & $\mathrm{C} 12$ & 1.3900 & C6 & $\mathrm{C} 7$ & $1.378(3)$ \\
\hline $\mathrm{C} 12$ & C11 & 1.3900 & & & \\
\hline
\end{tabular}

Table S13 Bond Angles for 6a.

$\begin{array}{cccc}\text { Atom } & \text { Atom } & \text { Atom } & \text { Angle }{ }^{\circ} \\ \text { C1 } & \text { N1 } & \text { C4 } & 11.39(13) \\ \text { C14 } & \text { C9 } & \text { C10 } & 120.0 \\ \text { C14 } & \text { C9 } & \text { C2 } & 119.70(8) \\ \text { C10 } & \text { C9 } & \text { C2 } & 120.18(8) \\ \text { C13 } & \text { C14 } & \text { C9 } & 120.0 \\ \text { C14 } & \text { C13 } & \text { C12 } & 120.0 \\ \text { C11 } & \text { C12 } & \text { C13 } & 120.0 \\ \text { C10 } & \text { C11 } & \text { C12 } & 120.0 \\ \text { C11 } & \text { C10 } & \text { C9 } & 120.0 \\ \text { C4 } & \text { C3 } & \text { C2 } & 108.75(13) \\ \text { C8 } & \text { C3 } & \text { C2 } & 130.69(15) \\ \text { C8 } & \text { C3 } & \text { C4 } & 120.53(15) \\ \text { O2 } & \text { C2 } & \text { C9 } & 108.92(11) \\ \text { O2 } & \text { C2 } & \text { C3 } & 115.70(12)\end{array}$

\begin{tabular}{cccc} 
Atom Atom & Atom & \multicolumn{1}{c}{ Angle $^{\circ}$} \\
O2 & C2 & C1 & $111.77(12)$ \\
C9 & C2 & C1 & $106.19(11)$ \\
C3 & C2 & C9 & $112.45(11)$ \\
C3 & C2 & C1 & $101.24(12)$ \\
O1 & C1 & N1 & $126.33(14)$ \\
O1 & C1 & C2 & $125.71(14)$ \\
N1 & C1 & C2 & $107.95(12)$ \\
C3 & C4 & N1 & $109.75(13)$ \\
C5 & C4 & N1 & $127.99(16)$ \\
C5 & C4 & C3 & $122.25(15)$ \\
C3 & C8 & C7 & $118.20(17)$ \\
C4 & C5 & C6 & $116.36(17)$ \\
C7 & C6 & C5 & $121.97(15)$ \\
C6 & C7 & C8 & $120.68(16)$
\end{tabular}


Table S14 Torsion Angles for 6a.

\begin{tabular}{|c|c|c|c|c|c|}
\hline $\mathbf{A}$ & B & D & Angle $/^{\circ}$ & A B C & Angle $/^{\circ}$ \\
\hline $\mathrm{O} 2$ & $\mathrm{C} 2$ & C1 O1 & $47.4(2)$ & C2 C9 C14 C13 & $175.94(10)$ \\
\hline $\mathrm{O} 2$ & $\mathrm{C} 2$ & $\mathrm{C} 1 \mathrm{~N} 1$ & $-133.23(13)$ & C2 C9 C10 C11 & $-175.92(10)$ \\
\hline N1 & $\mathrm{C} 4$ & C5 C6 & $-177.49(15)$ & $\mathrm{C} 2 \mathrm{C} 3 \mathrm{C} 4 \mathrm{~N} 1$ & $-3.81(17)$ \\
\hline C9 & $\mathrm{C} 14$ & C13 C12 & 0.0 & $\mathrm{C} 2 \mathrm{C} 3 \mathrm{C} 4 \mathrm{C} 5$ & $177.32(14)$ \\
\hline $\mathrm{C} 9$ & $\mathrm{C} 2$ & C1 O1 & $-71.24(17)$ & $\mathrm{C} 2 \mathrm{C} 3 \mathrm{C} 8 \mathrm{C} 7$ & $-178.17(15)$ \\
\hline $\mathrm{C} 9$ & $\mathrm{C} 2$ & C1 N1 & $108.12(13)$ & $\mathrm{C} 1 \mathrm{~N} 1 \mathrm{C} 4 \mathrm{C} 3$ & $-2.80(18)$ \\
\hline $\mathrm{C} 14$ & C9 & $\mathrm{C} 10 \mathrm{C} 11$ & 0.0 & $\mathrm{C} 1 \mathrm{~N} 1 \mathrm{C} 4 \mathrm{C} 5$ & $175.99(15)$ \\
\hline $\mathrm{C} 14$ & C9 & $\mathrm{C} 2 \quad \mathrm{O} 2$ & $160.50(9)$ & C4N1 C1 O1 & $-172 \cdot 72(14)$ \\
\hline C14 & $\mathrm{C} 9$ & $\mathrm{C} 2 \mathrm{C} 3$ & $30.87(14)$ & $\mathrm{C} 4 \mathrm{~N} 1 \mathrm{C} 1 \mathrm{C} 2$ & $7.93(17)$ \\
\hline $\mathrm{C} 14$ & $\mathrm{C} 9$ & $\mathrm{C} 2 \mathrm{C} 1$ & $-78.98(12)$ & $3 \mathrm{C} 2 \mathrm{O} 2$ & $128.85(14)$ \\
\hline C14 & $\mathrm{C} 13$ & C12 C11 & 0.0 & $\mathrm{C} 4 \mathrm{C} 3 \mathrm{C} 2 \mathrm{C} 9$ & $-105.11(14)$ \\
\hline C13 & $\mathrm{C} 12$ & $\mathrm{C} 11 \mathrm{C} 10$ & 0.0 & $\mathrm{C} 4 \mathrm{C} 3 \mathrm{C} 2 \mathrm{C} 1$ & $7.83(15)$ \\
\hline $\mathrm{C}$ & & C10 & 0.0 & $\mathrm{C} 4 \mathrm{C} 3 \mathrm{C} 8 \mathrm{C} 7$ & (2) \\
\hline $\mathrm{C} 10$ & C9 & $\mathrm{C} 14 \mathrm{C} 13$ & 0.0 & $\mathrm{C} 4 \mathrm{C} 5 \mathrm{C} 6 \mathrm{C} 7$ & $-0.1(2)$ \\
\hline $\mathrm{C} 10$ & C9 & $\mathrm{C} 2 \mathrm{O} 2$ & $-23.57(14)$ & $\mathrm{C} 8 \mathrm{C} 3 \mathrm{C} 2 \mathrm{O} 2$ & $-53.0(2)$ \\
\hline $\mathrm{C} 10$ & C9 & $\mathrm{C} 2 \mathrm{C} 3$ & $-153.19(10)$ & $\mathrm{C} 8 \mathrm{C} 3 \mathrm{C} 2 \mathrm{C} 9$ & $73.02(19)$ \\
\hline $\mathrm{C} 10$ & C9 & $\mathrm{C} 2 \mathrm{C} 1$ & $96.95(11)$ & $\mathrm{C} 8 \mathrm{C} 3 \mathrm{C} 2 \mathrm{C} 1$ & $-174.04(16)$ \\
\hline $\mathrm{C} 3$ & $\mathrm{C} 2$ & $\mathrm{C} 1 \mathrm{O} 1$ & $171.17(14)$ & $\mathrm{C} 8 \mathrm{C} 3 \mathrm{C} 4 \mathrm{~N} 1$ & $177.84(14)$ \\
\hline $\mathrm{C} 3$ & $\mathrm{C} 2$ & $\mathrm{C} 1 \mathrm{~N} 1$ & $-9.48(15)$ & $\mathrm{C} 8 \mathrm{C} 3 \mathrm{C} 4 \mathrm{C} 5$ & $-1.0(2)$ \\
\hline $\mathrm{C} 3$ & $\mathrm{C} 4$ & C5 C6 & $1.2(2)$ & $\mathrm{C} 5 \mathrm{C} 6 \mathrm{C} 7 \mathrm{C} 8$ & $-1.2(3)$ \\
\hline $\mathrm{C} 3$ & $\mathrm{C} 8$ & C7 C6 & $1.3(3)$ & & \\
\hline
\end{tabular}

Table S15 Hydrogen Atom Coordinates $\left(\AA \times 10^{4}\right)$ and Isotropic Displacement Parameters $\left(\AA^{2} \times 10^{3}\right)$ for 6 .

$\begin{array}{ccccc}\text { Atom } & \boldsymbol{x} & \boldsymbol{y} & \boldsymbol{z} & \mathbf{U}(\mathbf{e q}) \\ \text { H14 } & 6591.35 & 8073.03 & 6413.17 & 51 \\ \text { H13 } & 7608.69 & 9727.02 & 7021.14 & 60 \\ \text { H12 } & 6407 & 11516.12 & 7538.3 & 65 \\ \text { H11 } & 4187.95 & 11651.26 & 7447.49 & 66 \\ \text { H10 } & 3170.58 & 9997.28 & 6839.53 & 55 \\ \text { H8 } & 4508.27 & 4521.96 & 6566.02 & 55 \\ \text { H5 } & 6707.24 & 5411.79 & 5049.28 & 57 \\ \text { H6 } & 6868.55 & 2805.17 & 5483.43 & 64 \\ \text { H7 } & 5768 & 2350.03 & 6217.28 & 64 \\ \text { H1 } & 5479(19) & 8580(30) & 5077(9) & 55(6) \\ \text { H2 } & 2440(30) & 6980(40) & 6036(10) & 81(8)\end{array}$

\section{Experimental}

Single crystals of $\mathrm{C}_{14} \mathrm{H}_{11} \mathrm{NO}_{2}$ 6a were prepared by slow evaporation of saturated solution in EtOAc. A suitable crystal was selected and mounted on the glass stick by acrylic glue on a SuperNova, Dual, $\mathrm{Cu}$ at zero, AtlasS2 diffractometer. The crystal was kept at 302.56(10) K during data collection. Using Olex2 [S1], the structure was solved with the SHELXT [S2] structure solution program using Intrinsic Phasing and refined with the SHELXL [S3] refinement package using Least Squares minimization. Structures identical to compound 6a have been reported previously [S4-S6]. 


\section{Crystal structure determination of 6 a}

Crystal Data for $\mathrm{C}_{14} \mathrm{H}_{11} \mathrm{NO}_{2}(M=225.24 \mathrm{~g} / \mathrm{mol})$ : orthorhombic, space group Pbca (no. 61), $a=$ 10.3873(3) $\AA, b=7.7716(3) \AA, c=26.5900(10) \AA, V=2146.51(12) \AA^{3}, Z=8, T=302.56(10) \mathrm{K}, \mu(\mathrm{Cu} \mathrm{K \alpha})=0.763$ $\mathrm{mm}^{-1}$, Dcalc $=1.394 \mathrm{~g} / \mathrm{cm}^{3}, 11366$ reflections measured $\left(10.808^{\circ} \leq 2 \Theta \leq 152.706^{\circ}\right), 2223$ unique $\left(R_{\text {int }}=0.0805\right.$, $\left.\mathrm{R}_{\text {sigma }}=0.0408\right)$ which were used in all calculations. The final $R_{1}$ was $0.0499\left(\mathrm{I}>2 \sigma(\mathrm{I})\right.$ ) and $w R_{2}$ was 0.1485 (all data).

\section{Refinement model description}

Number of restraints - 0 , number of constraints - unknown.

Details:

1. Fixed Uiso

At 1.2 times of:

All C $(\mathrm{H})$ groups

2.a Aromatic/amide $\mathrm{H}$ refined with riding coordinates:

$\mathrm{C} 14(\mathrm{H} 14), \mathrm{C} 13(\mathrm{H} 13), \mathrm{C} 12(\mathrm{H} 12), \mathrm{C} 11(\mathrm{H} 11), \mathrm{C} 10(\mathrm{H} 10), \mathrm{C} 8(\mathrm{H} 8), \mathrm{C} 5(\mathrm{H} 5), \mathrm{C} 6(\mathrm{H} 6)$, $\mathrm{C} 7$ (H7)

2.b Fitted hexagon refined as free rotating group:

$\mathrm{C} 9(\mathrm{C} 14, \mathrm{C} 13, \mathrm{C} 12, \mathrm{C} 11, \mathrm{C} 10)$ 


\section{X-Ray crystallography data for $13 m$}
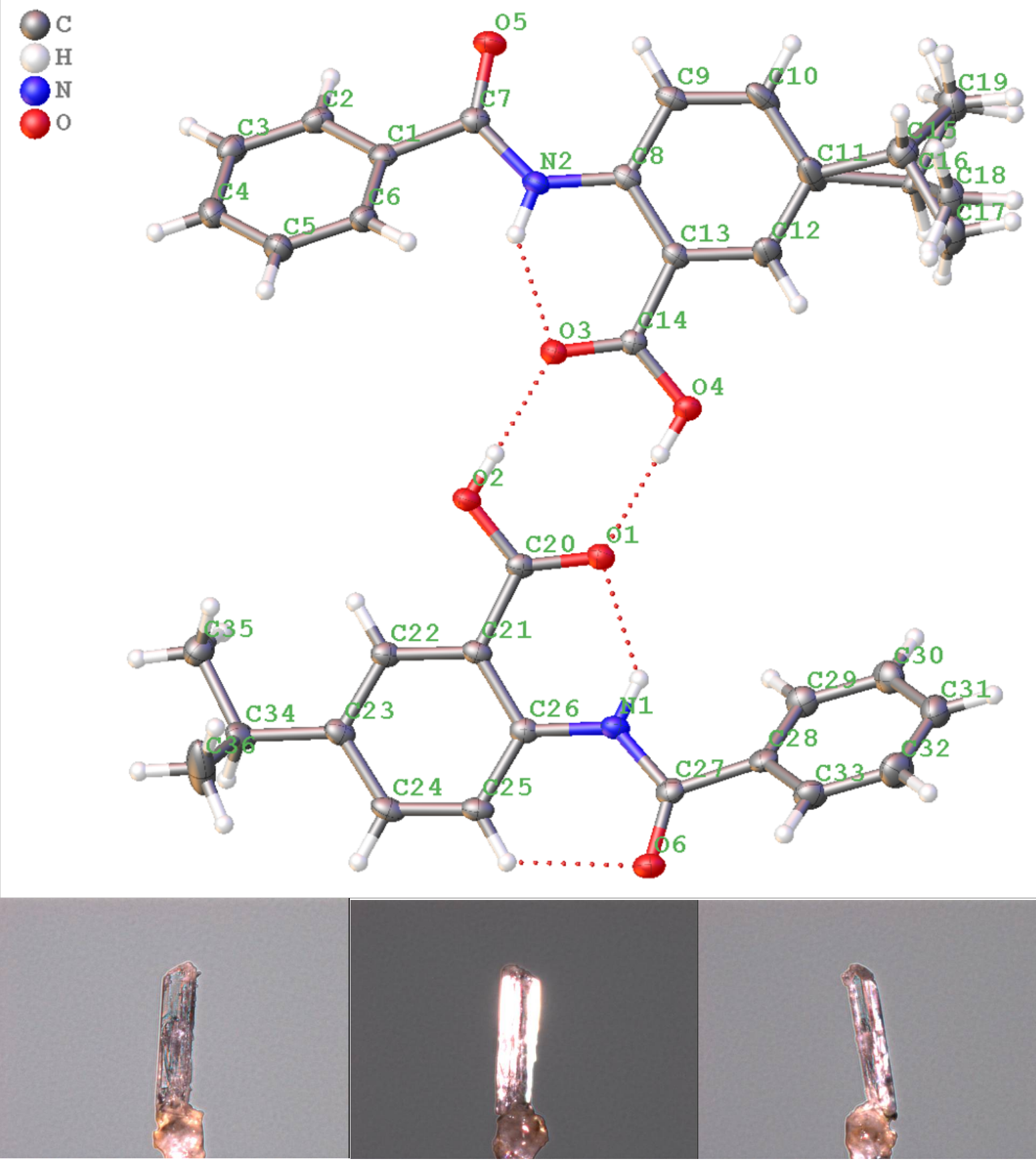

Figure S3. ORTEP drawing of the crystal structure showing 50\% probability thermal ellipsoids (top, CCDC 2105351) and microphotography of the single crystal of compound $\mathbf{1 3 m}$ used for XRay diffraction analysis at the bottom. 
Table S16 Crystal data and structure refinement for $13 \mathrm{~m}$.

Identification code

ANNA_ALV140_7

Empirical formula

$\mathrm{C}_{17} \mathrm{H}_{17} \mathrm{NO}_{3}$

Formula weight

283.31

Temperature/K

99.99(10)

Crystal system

orthorhombic

Space group

Pca2 1

$\begin{array}{ll}\mathrm{a} / \AA & 16.5446(3) \\ \mathrm{b} / \AA & 6.68250(10) \\ \mathrm{c} / \AA & 26.0422(6) \\ \alpha /^{\circ} & 90 \\ \beta /{ }^{\circ} & 90 \\ \gamma /{ }^{\circ} & 90 \\ \mathrm{Volume} / \AA^{3} & 2879.21(9) \\ \mathrm{Z} & 8 \\ \rho_{\text {calcg }} / \mathrm{cm}^{3} & 1.307 \\ \mu / \mathrm{mm}^{-1} & 0.729 \\ \mathrm{~F}(000) & 1200.0 \\ \mathrm{Crystal} \text { size } / \mathrm{mm}^{3} & 0.52 \times 0.151 \times 0.094 \\ \text { Radiation } & \mathrm{Cu} \mathrm{K \alpha}(\lambda=1.54184)\end{array}$

$2 \Theta$ range for data collection $/{ }^{\circ} 6.788$ to 152.786

Index ranges

$-20 \leq \mathrm{h} \leq 20,-5 \leq \mathrm{k} \leq 8,-29 \leq 1 \leq 32$

Reflections collected

16520

Independent reflections

$5127\left[\mathrm{R}_{\text {int }}=0.0368, \mathrm{R}_{\text {sigma }}=0.0350\right]$

Data/restraints/parameters

$5127 / 1 / 381$

Goodness-of-fit on $\mathrm{F}^{2}$

1.015

Final $R$ indexes $[\mathrm{I}>=2 \sigma(\mathrm{I})] \quad \mathrm{R}_{1}=0.0366, \mathrm{wR}_{2}=0.0916$

Final $\mathrm{R}$ indexes [all data] $\quad \mathrm{R}_{1}=0.0380, \mathrm{wR}_{2}=0.0931$

Largest diff. peak/hole / e $\AA^{-3} 0.24 /-0.22$

Flack parameter $\quad 0.19(9)$ 
Table S17 Fractional Atomic Coordinates $\left(\times 10^{4}\right)$ and Equivalent Isotropic Displacement Parameters $\left(\AA^{2} \times 10^{3}\right)$ for $6 \mathrm{~m}$. $U_{\text {eq }}$ is defined as $1 / 3$ of of the trace of the orthogonalised UIJ tensor.

\begin{tabular}{|c|c|c|c|c|}
\hline Atom & $x$ & $y$ & $z$ & $\mathbf{U}(\mathbf{e q})$ \\
\hline $\mathrm{O} 4$ & $6486.1(10)$ & $5902(2)$ & $5516.7(7)$ & $20.1(3)$ \\
\hline $\mathrm{O} 2$ & $5516.3(11)$ & $-874(2)$ & $4559.8(7)$ & $20.7(3)$ \\
\hline $\mathrm{O} 3$ & $6367.5(11)$ & $5834(2)$ & $4663.9(7)$ & $20.5(3)$ \\
\hline $\mathrm{O} 1$ & $5662.9(10)$ & $-738(2)$ & $5412.2(7)$ & $20.6(3)$ \\
\hline O5 & $6985.9(11)$ & $-58(2)$ & $3625.7(8)$ & $26.0(4)$ \\
\hline O6 & $5189.8(12)$ & $5547(2)$ & $6348.4(7)$ & $24.9(4)$ \\
\hline $\mathrm{N} 2$ & $6831.2(12)$ & $2592(3)$ & $4178.8(8)$ & $18.7(4)$ \\
\hline N1 & $5240.3(12)$ & $2634(3)$ & $5878.2(8)$ & $18.5(4)$ \\
\hline $\mathrm{C} 14$ & $6599.7(13)$ & $5055(3)$ & $5067.2(9)$ & $16.3(4)$ \\
\hline $\mathrm{C} 13$ & $7023.0(13)$ & $3099(3)$ & $5089.6(10)$ & $17.3(4)$ \\
\hline $\mathrm{C} 21$ & $4969.9(13)$ & $1924(3)$ & $4978.9(10)$ & $17.5(4)$ \\
\hline $\mathrm{C} 20$ & $5412.9(13)$ & $-1(3)$ & $5005.5(10)$ & $16.8(4)$ \\
\hline $\mathrm{C} 7$ & $6779.3(14)$ & $1669(3)$ & $3712.3(10)$ & $19.4(4)$ \\
\hline $\mathrm{C} 8$ & $7132.1(13)$ & $1902(3)$ & $4645.7(10)$ & $17.1(4)$ \\
\hline C34 & $3825.7(15)$ & $4882(3)$ & $3937.3(11)$ & $25.3(5)$ \\
\hline $\mathrm{C} 26$ & $4888.2(13)$ & $3191(3)$ & $5410.3(10)$ & $17.4(4)$ \\
\hline C9 & $7542.5(14)$ & $75(3)$ & $4700.0(11)$ & $21.0(5)$ \\
\hline C12 & $7305.4(16)$ & $2431(3)$ & $5566.9(11)$ & $23.7(5)$ \\
\hline $\mathrm{C} 1$ & $6442.2(9)$ & $3032.5(18)$ & $3301.7(5)$ & $19.0(4)$ \\
\hline C6 & $6625.2(9)$ & $5062.4(19)$ & $3294.4(6)$ & $21.3(5)$ \\
\hline $\mathrm{C} 5$ & $6328.6(10)$ & $6270.7(15)$ & $2902.9(7)$ & $24.4(5)$ \\
\hline $\mathrm{C} 4$ & $5849.0(11)$ & $5449(2)$ & $2518.7(6)$ & $26.8(5)$ \\
\hline $\mathrm{C} 3$ & $5666.0(10)$ & $3419(2)$ & $2526.0(6)$ & $24.7(5)$ \\
\hline $\mathrm{C} 2$ & $5962.6(10)$ & $2210.9(15)$ & $2917.5(6)$ & $22.6(5)$ \\
\hline $\mathrm{C} 28$ & $5879.7(9)$ & $2735(2)$ & $6708.4(5)$ & $19.7(5)$ \\
\hline $\mathrm{C} 33$ & $6442.0(10)$ & $3869.9(16)$ & $6977.5(7)$ & $26.1(5)$ \\
\hline $\mathrm{C} 32$ & $6899.3(10)$ & $2993(2)$ & $7364.6(7)$ & $31.9(6)$ \\
\hline $\mathrm{C} 31$ & $6794.2(10)$ & $981(2)$ & $7482.7(6)$ & $28.9(6)$ \\
\hline $\mathrm{C} 30$ & $6231.8(10)$ & $-153.7(16)$ & $7213.6(7)$ & $26.7(5)$ \\
\hline $\mathrm{C} 29$ & $5774.6(9)$ & $723(2)$ & $6826.4(6)$ & $24.6(5)$ \\
\hline $\mathrm{C} 23$ & $4198.2(14)$ & 4247 ( 3 ) & $4448.6(10)$ & $20.3(5)$ \\
\hline $\mathrm{C} 27$ & $5397.5(14)$ & $3797(3)$ & $6298.5(10)$ & $19.6(4)$ \\
\hline $\mathrm{C} 22$ & $4629.0(14)$ & $2483(3)$ & $4507.0(10)$ & $18.8(4)$ \\
\hline $\mathrm{C} 10$ & $7819.0(15)$ & $-534(3)$ & $5180.8(12)$ & $25.9(5)$ \\
\hline $\mathrm{C} 24$ & $4122.8(14)$ & $5475(3)$ & $4880.1(11)$ & $21.3(5)$ \\
\hline $\mathrm{C} 25$ & $4454.4(13)$ & $4985(3)$ & $5349.2(10)$ & $19.7(5)$ \\
\hline C19 & $7989.0(17)$ & $-2222(4)$ & $6248.8(12)$ & $28.9(5)$ \\
\hline $\mathrm{C} 35$ & $3802.4(16)$ & $3239(4)$ & $3534.3(11)$ & $27.8(5)$ \\
\hline $\mathrm{C} 11$ & $7696.7(18)$ & $608(3)$ & $5624.2(12)$ & $29.9(6)$ \\
\hline $\mathrm{C} 16$ & $7897(3)$ & $-13(5)$ & $6189.1(16)$ & $15.3(12)$ \\
\hline $\mathrm{C} 18$ & $8672(4)$ & $1057(8)$ & $6344(2)$ & $26.9(12)$ \\
\hline $\mathrm{C} 15$ & $8298(8)$ & $-46(12)$ & $6025(5)$ & $27(3)$ \\
\hline $\mathrm{C} 17$ & $8310(12)$ & 1435 ( 18$)$ & $6457(5)$ & $34(3)$ \\
\hline
\end{tabular}


Table S17 Fractional Atomic Coordinates $\left(\times 10^{4}\right)$ and Equivalent Isotropic Displacement Parameters $\left(\AA^{2} \times 10^{3}\right)$ for $6 \mathrm{~m}$. $U_{\text {eq }}$ is defined as $1 / 3$ of of the trace of the orthogonalised UIJ tensor.

$\begin{array}{ccccc}\text { Atom } & \boldsymbol{x} & \boldsymbol{y} & \boldsymbol{z} & \mathbf{U}(\mathbf{e q}) \\ \mathrm{C} 36 & 4259(3) & 6727(5) & 3725.2(15) & 55.2(11)\end{array}$

Table S18 Anisotropic Displacement Parameters $\left(\AA^{2} \times 10^{3}\right)$ for $6 \mathrm{~m}$. The Anisotropic displacement factor exponent takes the form: $-2 \pi^{2}\left[h^{2} a * 2 U_{11}+2 h k a * b * U_{12}+\ldots\right]$.

\begin{tabular}{|c|c|c|c|c|c|c|}
\hline Atom & $\mathbf{U}_{11}$ & $\mathbf{U}_{22}$ & $\mathbf{U}_{33}$ & $\mathbf{U}_{23}$ & $\mathbf{U}_{13}$ & $\mathbf{U}_{12}$ \\
\hline O4 & $24.2(8)$ & $15.6(7)$ & $20.5(9)$ & $-2.1(6)$ & $-1.6(7)$ & $1.5(6)$ \\
\hline $\mathrm{O} 2$ & $22.6(8)$ & $16.9(7)$ & $22.6(9)$ & $-2.7(6)$ & $-3.0(7)$ & $5.8(6)$ \\
\hline $\mathrm{O} 3$ & $24.8(8)$ & $17.3(7)$ & $19.5(9)$ & $-0.7(6)$ & $-0.3(7)$ & $4.6(6)$ \\
\hline $\mathrm{O} 1$ & $24.0(8)$ & $16.1(7)$ & $21.6(9)$ & $-0.9(6)$ & $-1 \cdot 3(7)$ & $1.7(6)$ \\
\hline O5 & $32.0(9)$ & $16.9(7)$ & $29.0(10)$ & $-4.2(7)$ & $-2 \cdot 3(8)$ & $5.5(6)$ \\
\hline O6 & $30.6(9)$ & $19.5(7)$ & $24.6(9)$ & $-4.7(7)$ & $0.9(7)$ & $4.8(7)$ \\
\hline $\mathrm{N} 2$ & $20.5(9)$ & $15.0(7)$ & $20.5(10)$ & $0.7(7)$ & $1.1(8)$ & $3.5(7)$ \\
\hline N1 & $18.8(9)$ & $14.2(8)$ & $22.6(11)$ & $-2.4(7)$ & $0.0(8)$ & $1.3(6)$ \\
\hline C14 & $13.3(9)$ & $15.4(9)$ & $20.2(11)$ & $-0.5(8)$ & 0.7 ( 8 ) & $-2.5(7)$ \\
\hline $\mathrm{C} 13$ & $14.3(9)$ & $14.8(9)$ & $22.8(12)$ & $0.3(8)$ & $-1.1(9)$ & $-1 \cdot 3(8)$ \\
\hline $\mathrm{C} 21$ & $13.9(9)$ & $14.6(9)$ & $23.9(12)$ & $-0.8(8)$ & $1.6(9)$ & $0.4(7)$ \\
\hline $\mathrm{C} 20$ & $14.0(9)$ & $14.9(9)$ & $21.6(12)$ & $-2.5(8)$ & $-0.3(9)$ & $-2.0(7)$ \\
\hline $\mathrm{C} 7$ & $16.4(10)$ & $18.7(9)$ & $23.3(12)$ & $-1.9(9)$ & $0.9(9)$ & $-0.5(8)$ \\
\hline $\mathrm{C} 8$ & $12.4(9)$ & $15.3(9)$ & $23.6(12)$ & $1.1(8)$ & $1.4(9)$ & $-1.5(7)$ \\
\hline C34 & $21.6(11)$ & $23.2(10)$ & $31.1(14)$ & $-4.1(10)$ & $-9.1(10)$ & $7.4(9)$ \\
\hline $\mathrm{C} 26$ & $13.7(9)$ & $16.6(9)$ & $21.8(11)$ & $-0.9(9)$ & $1.9(9)$ & $-2.0(8)$ \\
\hline C9 & $16.9(10)$ & $14.7(9)$ & $31.4(13)$ & $-0.4(9)$ & $0.6(9)$ & $1.3(8)$ \\
\hline $\mathrm{C} 12$ & $28.9(12)$ & $16.1(10)$ & $26.2(13)$ & $-2.2(9)$ & $-7.3(10)$ & $-3.3(9)$ \\
\hline $\mathrm{C} 1$ & $17.1(10)$ & $19.2(9)$ & $20.5(12)$ & $-2.9(9)$ & $2.5(9)$ & $3.2(8)$ \\
\hline C6 & $21.5(11)$ & $20.6(10)$ & $21.8(12)$ & $-3.5(9)$ & $-3.0(9)$ & $1.8(8)$ \\
\hline $\mathrm{C} 5$ & $30.6(12)$ & $18.5(10)$ & $24.2(13)$ & $0.2(9)$ & $-2.2(10)$ & $2.3(9)$ \\
\hline $\mathrm{C} 4$ & $26.5(12)$ & $29.8(11)$ & $24.0(13)$ & $0.0(10)$ & $-4.0(10)$ & $7.8(10)$ \\
\hline $\mathrm{C} 3$ & $20.8(11)$ & $30.5(11)$ & $22.9(13)$ & $-6.8(10)$ & $-3.4(9)$ & $1.4(9)$ \\
\hline $\mathrm{C} 2$ & $22.1(10)$ & $20.9(10)$ & $24.7(13)$ & $-6.6(9)$ & $0.5(10)$ & $-0.8(8)$ \\
\hline $\mathrm{C} 28$ & $20.0(10)$ & $20.7(10)$ & $18.5(12)$ & $-4.2(9)$ & $5.1(9)$ & $2.4(8)$ \\
\hline $\mathrm{C} 33$ & $28.1(12)$ & $21.0(10)$ & $29.3(13)$ & $-5.1(10)$ & $-2.1(11)$ & $-1.6(9)$ \\
\hline $\mathrm{C} 32$ & $31.5(14)$ & $31.2(12)$ & $33.0(15)$ & $-6.2(11)$ & $-9.8(12)$ & $-2.1(10)$ \\
\hline $\mathrm{C} 31$ & $27.9(13)$ & $33.3(13)$ & $25.4(14)$ & $-0.8(10)$ & $-3.9(11)$ & $5.2(10)$ \\
\hline $\mathrm{C} 30$ & $29.7(13)$ & $24.0(10)$ & $26.3(13)$ & $4.1(10)$ & $2.8(11)$ & $-0.9(9)$ \\
\hline $\mathrm{C} 29$ & $24.5(11)$ & $22.7(10)$ & $26.8(13)$ & $-0.3(10)$ & $1.5(10)$ & $-3.9(9)$ \\
\hline $\mathrm{C} 23$ & $13.8(10)$ & $19.0(10)$ & $28.0(13)$ & $-1.3(9)$ & $-3.0(9)$ & $0.9(8)$ \\
\hline $\mathrm{C} 27$ & $19.3(10)$ & $20.9(10)$ & $18.7(11)$ & $-1.3(9)$ & $5.0(9)$ & $-0.3(8)$ \\
\hline $\mathrm{C} 22$ & $15.0(9)$ & $17.5(9)$ & $23.9(12)$ & $-3.2(9)$ & $-0.9(9)$ & $-0.6(8)$ \\
\hline $\mathrm{C} 10$ & $20.8(11)$ & $14.7(9)$ & $42.3(16)$ & $0.4(10)$ & $-11.1(11)$ & $2.3(8)$ \\
\hline $\mathrm{C} 24$ & $14.4(10)$ & $17.0(9)$ & $32.4(14)$ & $-1.0(9)$ & $0.7(9)$ & $2.4(8)$ \\
\hline $\mathrm{C} 25$ & $16.1(10)$ & $15.7(9)$ & $27.4(13)$ & $-3.2(8)$ & $3.2(9)$ & 0.7 ( 8 ) \\
\hline
\end{tabular}


Table S18 Anisotropic Displacement Parameters $\left(\AA^{2} \times 10^{3}\right)$ for $6 \mathrm{~m}$. The Anisotropic displacement factor exponent takes the form: $-2 \pi^{2}\left[h^{2} a * 2 U_{11}+2 h k a * b * U_{12}+\ldots\right]$.

$\begin{array}{ccccccc}\text { Atom } & \mathbf{U}_{11} & \mathbf{U}_{22} & \mathbf{U}_{33} & \mathbf{U}_{23} & \mathbf{U}_{13} & \mathbf{U}_{12} \\ \text { C19 } & 32.8(13) & 25.9(11) & 27.9(14) & 2.5(10) & -5.9(11) & -2.6(10) \\ \text { C35 } & 27.8(12) & 30.2(12) & 25.5(13) & -3.6(11) & -2.1(11) & 2.5(10) \\ \text { C11 } & 36.8(14) & 16.8(10) & 36.0(16) & 1.2(10) & -18.5(13) & -2.1(10) \\ \text { C16 } & 16(2) & 17.6(15) & 12.6(18) & -2.1(12) & 1.6(17) & 0.8(12) \\ \text { C18 } & 25(3) & 28(2) & 27(2) & 4.0(18) & -10(2) & -7.0(19) \\ \text { C15 } & 25(6) & 28(4) & 27(5) & 0(3) & -1(5) & 0(3) \\ \text { C17 } & 40(8) & 32(5) & 31(6) & 4(4) & -9(6) & -7(5) \\ \text { C36 } & 88(3) & 32.4(14) & 45(2) & 15.7(15) & -37(2) & -18.5(16)\end{array}$

Table S19 Bond Lengths for 13m.

\begin{tabular}{|c|c|c|c|c|c|}
\hline \multicolumn{2}{|c|}{ Atom Atom } & \multirow{2}{*}{$\begin{array}{l}\text { Length/Å } \\
1.314(3)\end{array}$} & \multicolumn{2}{|c|}{ Atom Atom } & \multirow{2}{*}{$\begin{array}{c}\text { Length/Å } \\
1.3900\end{array}$} \\
\hline $\mathrm{O} 4$ & $\mathrm{C} 14$ & & $\mathrm{C} 1$ & C6 & \\
\hline $\mathrm{O} 2$ & $\mathrm{C} 20$ & $1.310(3)$ & $\mathrm{C} 1$ & 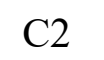 & 1.3900 \\
\hline $\mathrm{O} 3$ & $\mathrm{C} 14$ & ) & 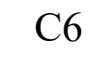 & & \\
\hline $\mathrm{O} 1$ & C & 3) & 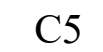 & & \\
\hline $\mathrm{O} 5$ & $\mathrm{C} 7$ & 3) & 4 & $C^{3}$ & 00 \\
\hline O6 & $\mathrm{C} 27$ & .2 & 3 & 2 & 00 \\
\hline $\mathrm{N} 2$ & & .3 & $\mathrm{C} 28$ & 33 & 00 \\
\hline N2 & 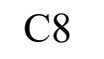 & & & & \\
\hline N1 & $\mathrm{C} 26$ & 1.40 & $\mathrm{c} 28$ & $\mathrm{C} 27$ & $1.510(3)$ \\
\hline N1 & $\mathrm{C} 27$ & .36 & 33 & $\mathrm{C} 32$ & \\
\hline C14 & $\mathrm{C} 13$ & ) & 32 & $\mathrm{C} 31$ & \\
\hline C13 & $\mathrm{C} 8$ & .4 & 31 & $\mathrm{C} 30$ & 00 \\
\hline C13 & $\mathrm{C} 12$ & 1.4 & 30 & $\mathrm{C} 29$ & 00 \\
\hline $\mathrm{C} 21$ & $\mathrm{C} 20$ & 1.4 & 23 & $\mathrm{C} 22$ & $1.386(3)$ \\
\hline $\mathrm{C} 21$ & $\mathrm{C} 26$ & 1.4 & 23 & $\mathrm{C} 24$ & $1.397(4)$ \\
\hline $\mathrm{C} 21$ & $\mathrm{C} 22$ & & & & \\
\hline $\mathrm{C} 7$ & $\mathrm{C} 1$ & 1.5 & 24 & $\mathrm{C} 25$ & (4) \\
\hline $\mathrm{C} 8$ & C9 & 1.4 & 19 & $\mathrm{C} 16$ & $1.492(4)$ \\
\hline C34 & $\mathrm{C} 23$ & 1.5 & C19 & $\mathrm{C} 15$ & $1.648(10)$ \\
\hline C34 & $\mathrm{C} 35$ & $1.519(4)$ & $\mathrm{C} 11$ & $\mathrm{C} 16$ & $1.564(5)$ \\
\hline C34 & $\mathrm{C} 36$ & $1.530(4)$ & $\mathrm{C} 11$ & $\mathrm{C} 15$ & $1.506(9)$ \\
\hline $\mathrm{C} 26$ & $\mathrm{C} 25$ & 1.40 & $\mathrm{C} 16$ & $\mathrm{C} 18$ & $1.524(7)$ \\
\hline C9 & $\mathrm{C} 10$ & $1.394(4)$ & $\mathrm{C} 15$ & $\mathrm{C} 17$ & 1.499 (19 \\
\hline $\mathrm{C} 12$ & $\mathrm{C} 11$ & $1.388(3)$ & & & \\
\hline
\end{tabular}

Table S20 Bond Angles for $13 \mathrm{~m}$.

\begin{tabular}{cccccccc}
\multicolumn{2}{c}{ Atom Atom Atom } & \multicolumn{1}{c}{ Angle $/^{\circ}$} & \multicolumn{4}{c}{ Atom Atom Atom } & Angle $/^{\circ}$ \\
C7 & N2 & C8 & $130.52(18)$ & C3 & C4 & C5 & 120.0 \\
C27 & N1 & C26 & $128.61(19)$ & C4 & C3 & C2 & 120.0
\end{tabular}


Table S20 Bond Angles for $13 \mathrm{~m}$.

\begin{tabular}{|c|c|c|c|c|c|c|c|}
\hline \multicolumn{3}{|c|}{ Atom Atom Atom } & \multirow{2}{*}{$\begin{array}{c}\text { Angle }^{\circ} \\
114.3(2)\end{array}$} & \multicolumn{3}{|c|}{ Atom Atom Atom } & \multirow{2}{*}{$\begin{array}{c}\text { Angle } /^{\circ} \\
120.0\end{array}$} \\
\hline $\mathrm{O} 4$ & $\mathrm{C} 14$ & $\mathrm{C} 13$ & & $\mathrm{C} 3$ & $\mathrm{C} 2$ & $\mathrm{C} 1$ & \\
\hline $\mathrm{O} 3$ & $\mathrm{C} 14$ & $\mathrm{O} 4$ & $122.16(19)$ & $\mathrm{C} 33$ & $\mathrm{C} 28$ & $\mathrm{C} 29$ & 120.0 \\
\hline $\mathrm{O} 3$ & $\mathrm{C} 14$ & $\mathrm{C} 13$ & $123.5(2)$ & $\mathrm{C} 33$ & $\mathrm{C} 28$ & $\mathrm{C} 27$ & $116.97(13)$ \\
\hline $\mathrm{C} 8$ & $\mathrm{C} 13$ & $\mathrm{C} 14$ & $121.7(2)$ & $\mathrm{C} 29$ & $\mathrm{C} 28$ & $\mathrm{C} 27$ & $123.02(13)$ \\
\hline $\mathrm{C} 12$ & $\mathrm{C} 13$ & $\mathrm{C} 14$ & $118.2(2)$ & $\mathrm{C} 28$ & C33 & C32 & 120.0 \\
\hline $\mathrm{C} 12$ & $\mathrm{C} 13$ & $\mathrm{C} 8$ & 120.09 (19) & C31 & C32 & C33 & 120.0 \\
\hline $\mathrm{C} 26$ & $\mathrm{C} 21$ & $\mathrm{C} 20$ & $122.0(2)$ & $\mathrm{C} 30$ & $\mathrm{C} 31$ & $\mathrm{C} 32$ & 120.0 \\
\hline $\mathrm{C} 22$ & $\mathrm{C} 21$ & $\mathrm{C} 20$ & $118.1(2)$ & $\mathrm{C} 29$ & $\mathrm{C} 30$ & C31 & 120.0 \\
\hline $\mathrm{C} 22$ & $\mathrm{C} 21$ & $\mathrm{C} 26$ & $119.88(19)$ & $\mathrm{C} 30$ & $\mathrm{C} 29$ & $\mathrm{C} 28$ & 120.0 \\
\hline $\mathrm{O} 2$ & $\mathrm{C} 20$ & $\mathrm{C} 21$ & $114.2(2)$ & $\mathrm{C} 22$ & $\mathrm{C} 23$ & C34 & $122.7(2)$ \\
\hline $\mathrm{O} 1$ & $\mathrm{C} 20$ & $\mathrm{O} 2$ & $122.49(19)$ & $\mathrm{C} 22$ & $\mathrm{C} 23$ & $\mathrm{C} 24$ & $117.2(2)$ \\
\hline $\mathrm{O} 1$ & $\mathrm{C} 20$ & $\mathrm{C} 21$ & $123.4(2)$ & $\mathrm{C} 24$ & $\mathrm{C} 23$ & $\mathrm{C} 34$ & $120.14(19)$ \\
\hline $\mathrm{O} 5$ & $\mathrm{C} 7$ & $\mathrm{~N} 2$ & $124.9(2)$ & O6 & $\mathrm{C} 27$ & $\mathrm{~N} 1$ & $125.0(2)$ \\
\hline $\mathrm{O} 5$ & $\mathrm{C} 7$ & $\mathrm{C} 1$ & $122.7(2)$ & O6 & $\mathrm{C} 27$ & $\mathrm{C} 28$ & $121.4(2)$ \\
\hline N2 & $\mathrm{C} 7$ & $\mathrm{C} 1$ & $112.36(17)$ & N1 & $\mathrm{C} 27$ & $\mathrm{C} 28$ & $113.51(18)$ \\
\hline N2 & $\mathrm{C} 8$ & $\mathrm{C} 13$ & 118.65 ( 18$)$ & $\mathrm{C} 23$ & $\mathrm{C} 22$ & $\mathrm{C} 21$ & $122.0(2)$ \\
\hline $\mathrm{N} 2$ & $\mathrm{C} 8$ & $\mathrm{C} 9$ & $123.3(2)$ & C9 & $\mathrm{C} 10$ & $\mathrm{C} 11$ & $122.4(2)$ \\
\hline C9 & $\mathrm{C} 8$ & $\mathrm{C} 13$ & $118.1(2)$ & $\mathrm{C} 25$ & $\mathrm{C} 24$ & $\mathrm{C} 23$ & $122.6(2)$ \\
\hline $\mathrm{C} 23$ & $\mathrm{C} 34$ & $\mathrm{C} 36$ & $110.5(2)$ & $\mathrm{C} 24$ & $\mathrm{C} 25$ & $\mathrm{C} 26$ & $120.4(2)$ \\
\hline $\mathrm{C} 35$ & $\mathrm{C} 34$ & $\mathrm{C} 23$ & $114.31(19)$ & $\mathrm{C} 12$ & $\mathrm{C} 11$ & $\mathrm{C} 10$ & $117.2(2)$ \\
\hline $\mathrm{C} 35$ & $\mathrm{C} 34$ & C36 & $110.2(3)$ & $\mathrm{C} 12$ & $\mathrm{C} 11$ & $\mathrm{C} 16$ & $115.6(3)$ \\
\hline N1 & $\mathrm{C} 26$ & $\mathrm{C} 21$ & 119.49 (19) & $\mathrm{C} 12$ & C11 & $\mathrm{C} 15$ & $129.6(4)$ \\
\hline N1 & $\mathrm{C} 26$ & $\mathrm{C} 25$ & $122.5(2)$ & $\mathrm{C} 10$ & C11 & $\mathrm{C} 16$ & $127.0(2)$ \\
\hline $\mathrm{C} 25$ & $\mathrm{C} 26$ & $\mathrm{C} 21$ & $118.0(2)$ & $\mathrm{C} 10$ & $\mathrm{C} 11$ & $\mathrm{C} 15$ & $108.5(5)$ \\
\hline $\mathrm{C} 10$ & $\mathrm{C} 9$ & $\mathrm{C} 8$ & $120.2(2)$ & C19 & $\mathrm{C} 16$ & $\mathrm{C} 11$ & $112.5(3)$ \\
\hline $\mathrm{C} 11$ & $\mathrm{C} 12$ & C13 & $122.0(2)$ & C19 & $\mathrm{C} 16$ & $\mathrm{C} 18$ & $110.5(4)$ \\
\hline C6 & $\mathrm{C} 1$ & $\mathrm{C} 7$ & $121.16(12)$ & $\mathrm{C} 18$ & $\mathrm{C} 16$ & C11 & $107.6(4)$ \\
\hline C6 & $\mathrm{C} 1$ & $\mathrm{C} 2$ & 120.0 & C11 & $\mathrm{C} 15$ & C19 & $107.2(6)$ \\
\hline $\mathrm{C} 2$ & $\mathrm{C} 1$ & $\mathrm{C} 7$ & $118.80(12)$ & C17 & $\mathrm{C} 15$ & C19 & $108.7(10)$ \\
\hline $\mathrm{C} 1$ & $\mathrm{C} 6$ & $\mathrm{C} 5$ & 120.0 & $\mathrm{C} 17$ & $\mathrm{C} 15$ & $\mathrm{C} 11$ & $109.7(10)$ \\
\hline C6 & $\mathrm{C} 5$ & $\mathrm{C} 4$ & 1 & & & & \\
\hline
\end{tabular}

Table S21 Hydrogen Bonds for 13m.

$\begin{array}{ccccccc}\mathbf{D} & \mathbf{H} & \mathbf{A} & \mathbf{d}(\mathbf{D}-\mathbf{H}) / \AA & \mathbf{d}(\mathbf{H}-\mathbf{A}) / \AA & \mathbf{d}(\mathbf{D}-\mathbf{A}) / \AA & \mathbf{D}-\mathbf{H}-\mathbf{A} /{ }^{\circ} \\ \mathrm{O} 4 & \mathrm{H} 4 & \mathrm{O}^{1} & 0.82 & 1.82 & 2.640(2) & 174.1 \\ \mathrm{O} 2 & \mathrm{H} 2 \mathrm{~B} & \text { O3 } & & & \\ & 0.82 & 1.81 & 2.626(2) & 176.7 \\ \mathrm{~N} 2 & \mathrm{H} 2 & \mathrm{O} 3 & 0.86 & 1.90 & 2.622(2) & 140.2 \\ \mathrm{~N} 1 & \mathrm{H} 1 & \mathrm{O} 1 & 0.86 & 1.98 & 2.653(2) & 134.8 \\ \mathrm{C} 25 & \mathrm{H} 25 & \mathrm{O} 6 & 0.93 & 2.31 & 2.897(3) & 121.0\end{array}$

${ }^{1}+\mathrm{X}, 1+\mathrm{Y},+\mathrm{Z} ;{ }^{2}+\mathrm{X},-1+\mathrm{Y},+\mathrm{Z}$ 
Table S22 Torsion Angles for 13m.

\begin{tabular}{|c|c|c|c|}
\hline $\begin{array}{llll}A & B & C & D\end{array}$ & Angle $/^{\circ}$ & $\mathbf{A}$ & Angle ${ }^{\circ}$ \\
\hline $\mathrm{O} 4 \mathrm{C} 14 \mathrm{C} 13 \mathrm{C} 8$ & $174.42(19)$ & $\mathrm{C} 12 \mathrm{C} 13 \mathrm{C} 8 \mathrm{C} 9$ & $-1.4(3)$ \\
\hline $4 \mathrm{C} 14 \mathrm{C} 13 \mathrm{C} 12$ & $-4 \cdot 5(3)$ & C12C11 C16C19 & $156.2(3)$ \\
\hline $3 \mathrm{C} 14 \mathrm{C} 13 \mathrm{C} 8$ & -5 & $\mathrm{C} 12 \mathrm{C} 11 \mathrm{C} 16 \mathrm{C} 18$ & $-81.9(5)$ \\
\hline $\mathrm{O} 3 \mathrm{C} 14 \mathrm{C} 13 \mathrm{C} 12$ & $175.8(2)$ & C12C11 C15C19 & $130.6(5)$ \\
\hline $\mathrm{O} 5 \mathrm{C} 7 \mathrm{C} 1 \mathrm{C} 6$ & $-143.23(19)$ & $\mathrm{C} 12 \mathrm{C} 11 \mathrm{C} 15 \mathrm{C} 17$ & $12.8(16)$ \\
\hline O5 $\quad$ C7 $\quad$ C1 $\quad$ C2 & 34. & $\mathrm{C} 1 \mathrm{C} \mathrm{C} 5 \mathrm{C} 4$ & 0.0 \\
\hline $\mathrm{N} 2 \quad \mathrm{C} 7 \quad \mathrm{C} 1 \quad \mathrm{C} 6$ & 36 & C6 $\quad \mathrm{C} 1 \quad \mathrm{C} 2 \mathrm{C} 3$ & 0.0 \\
\hline $\mathrm{N} 2 \quad \mathrm{C} 7 \quad \mathrm{C} 1 \quad \mathrm{C} 2$ & -145. & C6 $\quad \mathrm{C} 5 \mathrm{C}_{4} \mathrm{C} 3$ & 0.0 \\
\hline N2 $\quad$ C8 $89 \begin{array}{lll}C & C\end{array}$ & $-179.2(2)$ & $\begin{array}{llll}\mathrm{C} 5 & \mathrm{C} 4 & \mathrm{C} 3 & \mathrm{C} 2\end{array}$ & 0.0 \\
\hline N1 C26C25 C24 & $179.6(2)$ & C4 432 C2 $\quad$ C1 & 0.0 \\
\hline $14 \mathrm{C} 13 \mathrm{C} 8 \mathrm{~N} 2$ & -0 . & $\mathrm{C} 2 \mathrm{C} 1 \mathrm{C} 6 \mathrm{C} 5$ & 0.0 \\
\hline $14 \mathrm{C} 13 \mathrm{C} 8 \mathrm{C} 9$ & 179.7 & C28C33C32C31 & 0.0 \\
\hline 14 C13 C12 C11 & $179.0(2)$ & C33 C28 C29 C30 & 0.0 \\
\hline 13 C8 C9 C10 & 1.0 & C33C28C27 O6 & (3) \\
\hline $13 \mathrm{C} 12 \mathrm{C} 11 \mathrm{C} 10$ & 1. & 27 N1 & 143.87 \\
\hline $13 \mathrm{C} 12 \mathrm{C} 11 \mathrm{C} 16$ & -174 & C33 C32 C31 C30 & 0.0 \\
\hline $13 \mathrm{C} 12 \mathrm{C} 11 \mathrm{C} 15$ & 154 . & $30 \mathrm{C} 29$ & 0.0 \\
\hline $21 \mathrm{C} 26 \mathrm{C} 25 \mathrm{C} 24$ & -0 . & C31 C30 C29 C28 & 0 \\
\hline C20 C21 C26 N1 & 0.6 & C29C28C33 C32 & 0.0 \\
\hline $\mathrm{C} 20 \mathrm{C} 21 \mathrm{C} 26 \mathrm{C} 25$ & -179 & C29C28C27 O6 & 144.1 \\
\hline C20 C21 C22 C23 & 179.4 & $\mathrm{C} 29 \mathrm{C} 28 \mathrm{C} 27 \mathrm{~N} 1$ & $-37.0(2)$ \\
\hline C7 N2 C8 C13 & -172 . & $\mathrm{C} 23 \mathrm{C} 24 \mathrm{C} 25 \mathrm{C} 26$ & $-0.1(4)$ \\
\hline C7 $\mathrm{N} 2 \quad \mathrm{C} 8 \mathrm{C} 9$ & 7.8 & C27 N1 C26C21 & $163.3(2)$ \\
\hline C7 C1 C6 C5 & 177.8 & C27 N1 C26C25 & $-16.4(4)$ \\
\hline $\mathrm{C} 7 \mathrm{C} 1 \mathrm{C} 2 \mathrm{C} 3$ & -177.9 & C27 C28C33 C32 & $179.17(17)$ \\
\hline C8 $\quad \mathrm{N} 2 \mathrm{C}$ O5 & 2.0 & C27 C28C29C30 & $-179.12(1$ \\
\hline C8 $\quad \mathrm{N} 2 \mathrm{C}$ C1 & -177 & $\mathrm{C} 22 \mathrm{C} 21 \mathrm{C} 20 \mathrm{O} 2$ & $7.5(3)$ \\
\hline C8 C13 C12 C11 & $0.1(4)$ & $\mathrm{C} 22 \mathrm{C} 21 \mathrm{C} 20 \mathrm{O} 1$ & $-172.2(2)$ \\
\hline C8 C9 C10 C11 & 0. & C22 C21 C26 N1 & $-179.1(2)$ \\
\hline C34 C23 C22 C21 & 179.8 & $\mathrm{C} 22 \mathrm{C} 21 \mathrm{C} 26 \mathrm{C} 25$ & $0.6(3)$ \\
\hline C 34 C23 C24 C25 & $-179.3(2)$ & $\mathrm{C} 22 \mathrm{C} 23 \mathrm{C} 24$ & $-0.2(3)$ \\
\hline C26 N1 C27 O6 & 6.2 & C10C11 C16C19 & $-19.0(6)$ \\
\hline C26 N1 C27 C28 & $-172.6(2)$ & $\mathrm{C} 10 \mathrm{C} 11 \mathrm{C} 16 \mathrm{C} 18$ & $102.9(4)$ \\
\hline $\mathrm{C} 26 \mathrm{C} 21 \mathrm{C} 20 \mathrm{O} 2$ & $-172.1(2)$ & C10C11 C15C19 & $-74.8(9)$ \\
\hline $\mathrm{C} 26 \mathrm{C} 21 \mathrm{C} 20 \mathrm{O} 1$ & $8.2(3)$ & $\mathrm{C} 10 \mathrm{C} 11 \mathrm{C} 15 \mathrm{C} 17$ & $167.4(10)$ \\
\hline C26 C21 C22 C23 & $-1.0(3)$ & $\mathrm{C} 24 \mathrm{C} 23 \mathrm{C} 22 \mathrm{C} 21$ & $0.8(3)$ \\
\hline C9 C10C11 C12 & $-2.0(4)$ & C35 C34 C23 C22 & $12.9(3)$ \\
\hline C9 C10C11 C16 & $173.1(3)$ & C35 C34 C23 C24 & $-168.1(2)$ \\
\hline C9 C10C11 C15 & $-160.2(5)$ & C36C34 C23 C22 & $-112.0(3)$ \\
\hline $\mathrm{C} 12 \mathrm{C} 13 \mathrm{C} 8 \mathrm{~N} 2$ & $178.8(2)$ & C36C34 C23 C24 & $67.0(3)$ \\
\hline
\end{tabular}


Table S23 Hydrogen Atom Coordinates $\left(\AA \times 10^{4}\right)$ and Isotropic Displacement Parameters $\left(\AA^{2} \times 10^{3}\right)$ for $13 \mathrm{~m}$.

\begin{tabular}{|c|c|c|c|c|}
\hline Atom & $x$ & $y$ & $z$ & $\mathbf{U}(\mathbf{e q})$ \\
\hline $\mathrm{H} 4$ & 6260.39 & 6983.83 & 5477.22 & 30 \\
\hline $\mathrm{H} 2 \mathrm{~B}$ & 5767.92 & -1919.21 & 4599.65 & 31 \\
\hline $\mathrm{H} 2$ & 6648.19 & 3796.62 & 4185.42 & 22 \\
\hline H1 & 5375.65 & 1395.24 & 5904.59 & 22 \\
\hline H34 & 3265.27 & 5272.56 & 4006.52 & 30 \\
\hline H9 & 7630.08 & -731.74 & 4414.29 & 25 \\
\hline H12 & 7228.3 & 3235.17 & 5854.28 & 28 \\
\hline H6 & 6946.08 & 5612.19 & 3551.47 & 26 \\
\hline H5 & 6451.1 & 7628.81 & 2898.04 & 29 \\
\hline $\mathrm{H} 4 \mathrm{~A}$ & 5650.63 & 6257.42 & 2256.81 & 32 \\
\hline H3 & 5345.14 & 2869.4 & 2268.99 & 30 \\
\hline $\mathrm{H} 2 \mathrm{~A}$ & 5840.11 & 852.74 & 2922.41 & 27 \\
\hline H33 & 6512.37 & 5215.92 & 6898.54 & 31 \\
\hline H32 & 7275.57 & 3752.45 & 7544.69 & 38 \\
\hline H31 & 7100.13 & 394.63 & 7741.69 & 35 \\
\hline H30 & 6161.5 & -1499.74 & 7292.53 & 32 \\
\hline H29 & 5398.29 & -36.3 & 6646.38 & 30 \\
\hline H22 & 4694.21 & 1645.56 & 4224.84 & 23 \\
\hline H10 & 8094.65 & -1741.87 & 5207.75 & 31 \\
\hline $\mathrm{H} 24$ & 3837.81 & 6668.24 & 4849.67 & 26 \\
\hline $\mathrm{H} 25$ & 4390.84 & 5846.4 & 5626.87 & 24 \\
\hline H19A & 7507.08 & -2877.59 & 6131.12 & 43 \\
\hline H19B & 8077.12 & -2537.42 & 6603.99 & 43 \\
\hline $\mathrm{H} 19 \mathrm{C}$ & 8442.18 & -2671.42 & 6049.55 & 43 \\
\hline H19D & 7430.61 & -2126.11 & 6345.97 & 43 \\
\hline H19E & 8306.88 & -2581.15 & 6543.03 & 43 \\
\hline $\mathrm{H} 19 \mathrm{~F}$ & 8049.37 & -3225.35 & 5987.8 & 43 \\
\hline H35A & 4344.13 & 2838.83 & 3451.15 & 42 \\
\hline H35B & 3539.05 & 3729.56 & 3230.85 & 42 \\
\hline $\mathrm{H} 35 \mathrm{C}$ & 3509.35 & 2110.67 & 3666.11 & 42 \\
\hline H16 & 7457.68 & 442.82 & 6413.1 & 18 \\
\hline H18A & 8818.91 & 673.31 & 6686.67 & 40 \\
\hline H18B & 8588.73 & 2478.06 & 6330.97 & 40 \\
\hline $\mathrm{H} 18 \mathrm{C}$ & 9098.43 & 694.93 & 6111.51 & 40 \\
\hline H15 & 8837.9 & -173.93 & 5873.09 & 32 \\
\hline H17A & 7765.25 & 1755.87 & 6553.95 & 51 \\
\hline H17B & 8582.81 & 2629.3 & 6348.1 & 51 \\
\hline $\mathrm{H} 17 \mathrm{C}$ & 8588.73 & 867.18 & 6745.47 & 51 \\
\hline H36A & 4224.61 & 7798.22 & 3969.91 & 83 \\
\hline H36B & 4008.08 & 7131.29 & 3409.41 & 83 \\
\hline $\mathrm{H} 36 \mathrm{C}$ & 4816.53 & 6410.37 & 3663.09 & 83 \\
\hline
\end{tabular}


Table S24 Atomic Occupancy for $13 \mathrm{~m}$.

$\begin{array}{cccccc}\text { Atom } & \text { Occupancy } & \text { Atom } & \text { Occupancy } & \text { Atom } & \text { Occupancy } \\ \text { H19A } & 0.685(14) & \text { H19B } & 0.685(14) & \text { H19C } & 0.685(14) \\ \text { H19D } & 0.315(14) & \text { H19E } & 0.315(14) & \text { H19F } & 0.315(14) \\ \text { C16 } & 0.685(14) & \text { H16 } & 0.685(14) & \text { C18 } & 0.685(14) \\ \text { H18A } & 0.685(14) & \text { H18B } & 0.685(14) & \text { H18C } & 0.685(14) \\ \text { C15 } & 0.315(14) & \text { H15 } & 0.315(14) & \text { C17 } & 0.315(14) \\ \text { H17A } & 0.315(14) & \text { H17B } & 0.315(14) & \text { H17C } & 0.315(14)\end{array}$

\section{Experimental}

Single crystals of $\mathrm{C}_{17} \mathrm{H}_{17} \mathrm{NO}_{3} \mathbf{1 3 m}$ were prepared by slow evaporation of saturated solution in EtOAc. A suitable crystal was selected and mounted on the glass stick by acrylic glue on a SuperNova, Dual, Cu at home/near, AtlasS2 diffractometer. The crystal was kept at 99.99(10) K during data collection. Using Olex2 [S1], the structure was solved with the SHELXT [S2] structure solution program using Intrinsic Phasing and refined with the SHELXL [S3] refinement package using Least Squares minimization.

\section{Crystal structure determination of $13 \mathrm{~m}$}

Crystal Data for $\mathrm{C}_{17} \mathrm{H}_{17} \mathrm{NO}_{3}(M=283.31 \mathrm{~g} / \mathrm{mol})$ : orthorhombic, space group Pca2 $($ no. 29), $a=$ 16.5446(3) $\AA, b=6.68250(10) \AA, c=26.0422(6) \AA, V=2879.21(9) \AA^{3}, Z=8, T=99.99(10) \mathrm{K}, \mu(\mathrm{Cu} \mathrm{K} \alpha)=0.729$ $\mathrm{mm}^{-1}$, Dcalc $=1.307 \mathrm{~g} / \mathrm{cm}^{3}, 16520$ reflections measured $\left(6.788^{\circ} \leq 2 \Theta \leq 152.786^{\circ}\right), 5127$ unique $\left(R_{\text {int }}=0.0368\right.$, $\left.\mathrm{R}_{\text {sigma }}=0.0350\right)$ which were used in all calculations. The final $R_{1}$ was $0.0366(\mathrm{I}>2 \sigma(\mathrm{I}))$ and $w R_{2}$ was 0.0931 (all data).

\section{Refinement model description}

Details:

Number of restraints - 1, number of constraints - unknown.

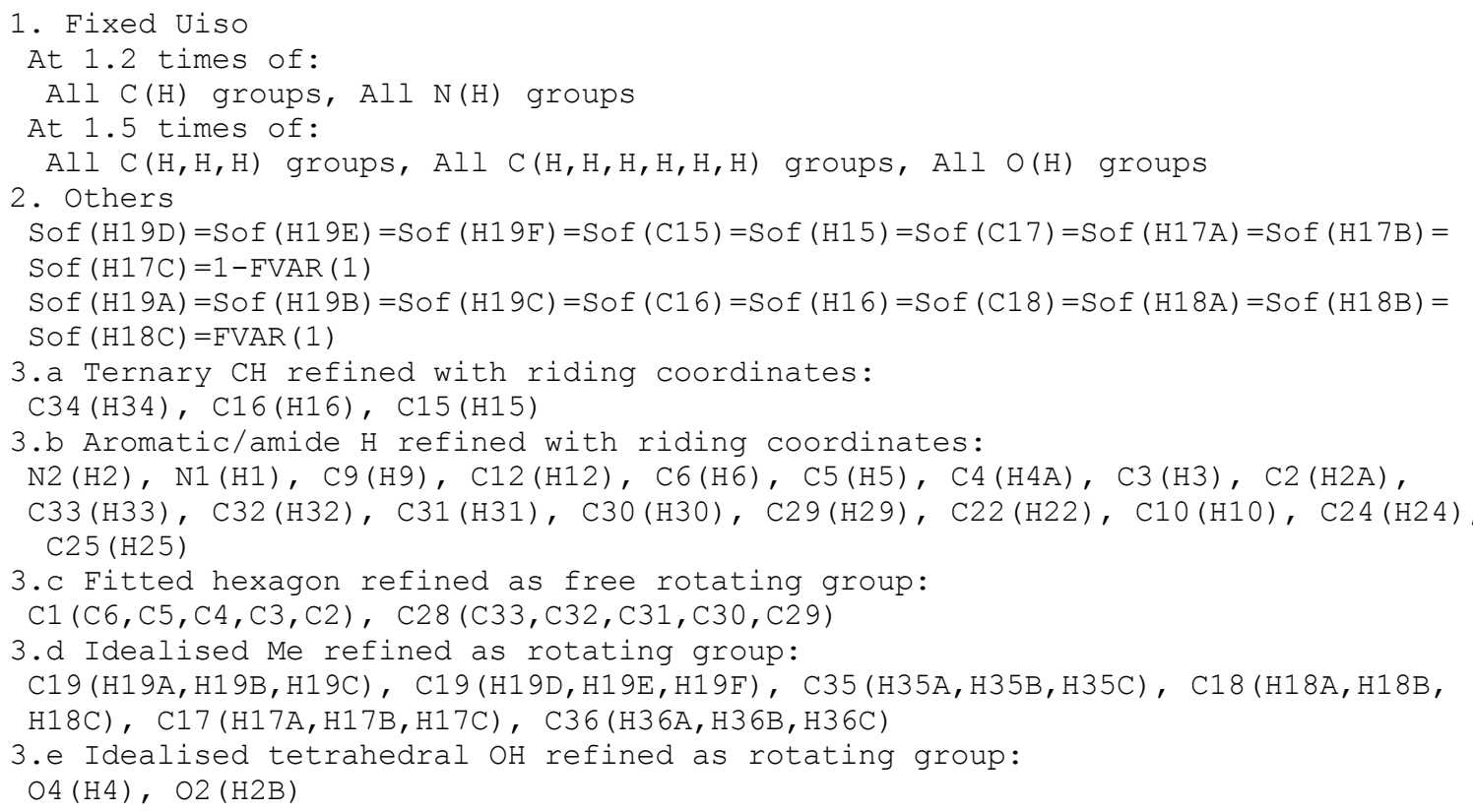




\section{References}

(S1) Dolomanov, O.V., Bourhis, L. J., Gildea, R.J, Howard, J. A .K. \& Puschmann, H. OLEX2: a Complete Structure Solution, Refinement and Analysis Program. J. Appl. Cryst. 2009, 42, 339341.

(S2) Sheldrick, G.M. SHELXT - Integrated Space-Group and Crystal-Structure Determination. Acta Cryst. 2015, A71, 3-8.

(S3) Sheldrick, G.M. Crystal Structure Refinement with SHELXL. Acta Cryst. 2015, C71, 3-8. (S4) Zhao, Y., Zhu, H., Sung, S., Wink, D. J., Zadrozny, J. M., Driver, T. G. Counterion Control of t-BuO-Mediated Single Electron Transfer to Nitrostilbenes to Construct $N$-Hydroxyindoles or Oxindoles. Angew.Chem.,Int.Ed. 2021, 60, 19207-19213.

(S5) Xiao, Z.-K., Lu, J.-M. Crystal structure of 3-hydroxy-3-phenyl-1,3-dihydro-2H-indol-2-one, $\mathrm{C}_{14} \mathrm{H}_{11} \mathrm{NO}_{2}$. Z.Kristallogr.-New Cryst.Struct. 2016, 231, 719-720.

(S6) Zhou, Z., Xu, Y., Zhu, B., Li, P., Hu, G., Yang, F., Xu, S., Zhang, X. One-pot synthesis of 3-hydroxy-2-oxindoles via acyloin rearrangements of 2-hydroxy-indolin-3-ones generated in situ from 2-alkynyl arylazides. New J.Chem., 2020, 44, 20303-2030. 